\title{
MODELLING OF SUBMERGED OSCILLATING WATER COLUMNS WITH MASS TRANSFER FOR WAVE ENERGY EXTRACTION
}

BY:

Sjors de Rooij

16 JULY 2019

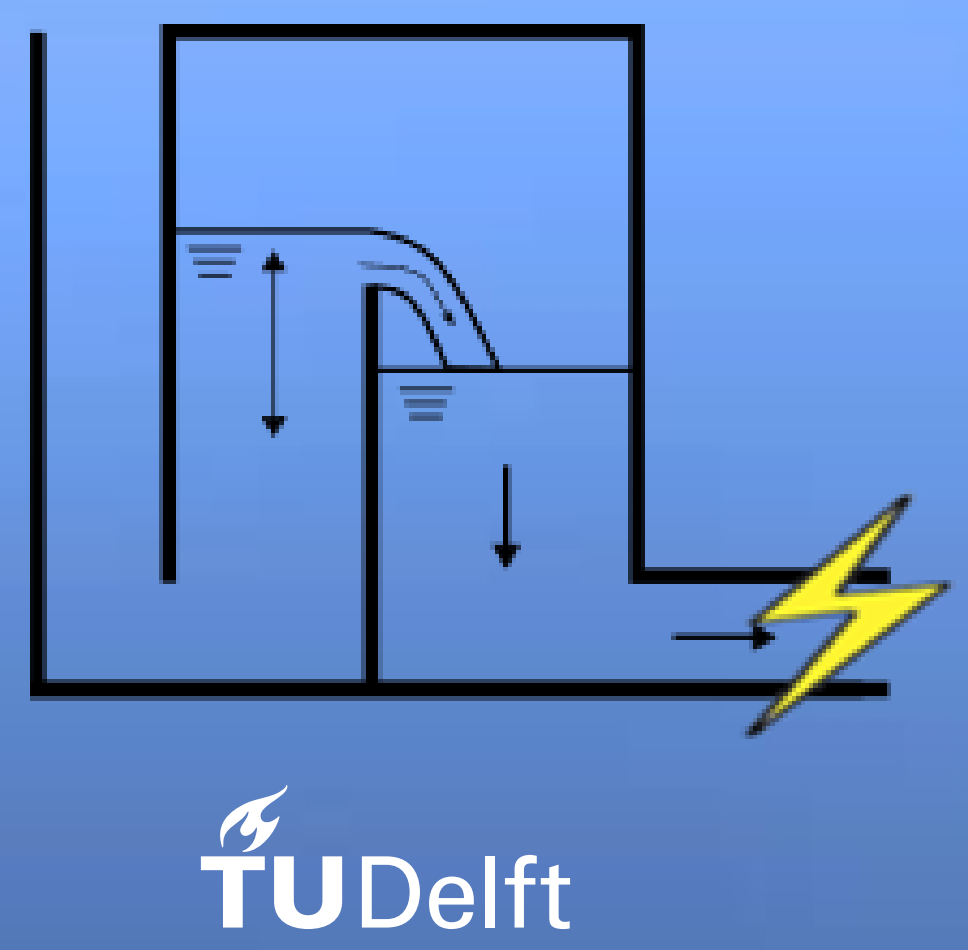





\title{
Modelling of submerged oscillating water columns with mass transfer for wave energy extraction
}

\author{
Sjors P.A. de Rooij
}

to obtain the degree of Master of Science at the Delft University of Technology,

to be defended publicly on Tuesday July 16, 2019 at 13:00 AM.

\author{
Student number: $\quad 4168429$ \\ Project duration: $\quad$ September 1, 2018 - July 16, 2019 \\ Thesis committee: Prof. dr. ir. A.V. Metrikine, TU Delft \\ Dr. ir. A. Jarquin-Laguna, TU Delft \\ Ir. A.J. Göbel,
}

An electronic version of this thesis is available at http://repository.tudelft.nl/. 



\section{Abstract}

In the past decades, the global demand for energy has increased. The aim to reduces has led to an increase in the production of renewable energy sources, such as wind and solar energy. However, there are more major energy resources available in the oceans, such as wave and tidal power. The estimated potential global energy resource in waves is around 2 Terra Watt. Over the years, many different technologies have been developed to harvest this high-density energy source. Yet, the harsh weather conditions are challenging the survival of the energy converters.

A potential device that avoids the risk of high environmental impacts and has a reduced vulnerability is the Neptune: The Neptune is a fully submerged wave energy converter. Inside the structure, a weir and internal air pocket separate two water columns. One column acts as an oscillating water column to pressures of the incident waves. During its oscillations, the inner free surface level exceeds the weir and spills water into the second column. This column acts as a reservoir, and the overflow water is drawn off through an exit pipe, including a turbine. From the net flow through the columns, energy can be extracted. This design has the advantage of being fully submerged and has a single moving part, namely the turbine.

The first work on this type of wave energy converter dates back to the mid-1970s. In 2008 a $1 / 20^{\text {th }}$ scale model was built and tested in a wave basin. However, the work and experiment didn't include the effects of energy generation. The objective of this thesis is to form a more scientific base concerning this device.

A numerical model is made to predict the dynamic behaviour of the system. The equations of motion of the water columns are derived from the equations of conservation of mass and momentum. The excitation forces are obtained from the linear wave theory for regular undisturbed waves. The hydrodynamic coefficients are determined from associated literature. The dynamics of the internal air pressure is derived from the conservation of mass and the pressure density relation for an adiabatic and reversible thermodynamic process. The pressure oscillation of the air chamber is coupled to the flow of the columns. The mass transfer between the columns is assumed instantaneous and modelled for the different conditions of the free surface displacement with respect to the weir level. The weir discharge is included in the expressions for the convective acceleration and momentum associated with the change of mass in the columns. An impact pressure is derived for the falling water from the weir on the column. The response of the turbine was modelled assuming a linear relationship between the flow through the turbine and the regression relation of stationary hydraulic turbines.

Using the developed model, the time series of the response is obtained by solving the non-linear differential equations in the Matlab ODE45 solver. The model is solved for a full-scale structure with a natural period of 8.5 seconds. The results are found for different periods of regular incident waves and various weir levels. From the results, an increase of resonance periods was identified, caused by the transfer of potential energy from the resonating column to the other column. Due to the weir discharge, a uni-directional flow is obtained in the second column. The uni-directional flow is desired for the extraction of energy. Furthermore, an increase in the mean displacement in the first column is found, caused by the damping in the second column. The increased of damping, from pressure losses or the turbine, resulted in a decrease of weir discharge. An optimal turbine diameter was found to be dependent on the relation between the dynamic flow and the damping induced by the turbine. This relation also results in a maximum power take-off efficiency of 7 to $12 \%$ depending on the weir level and the incident wave height. These are low efficiencies compared to other wave energy converters. It is likely caused by the inefficient energy transfer between the columns. Further research and optimizations are required to assess the feasibility of this wave energy converter.

Lastly, the numerical model is validated with the scale experiments. The amplitude of the response was found to be estimated well in the numerical model. However, in the model, the peak periods were underestimated together with the response for higher wave periods. 



\section{Acknowledgments}

Firstly, I would like to thank the people who have supported me during my graduation. Without the mental and practical support, I would not have been able to complete this journey to my graduation. First of all, I want to thank professor Andrei Metrikine for all the help and guidance. My special appreciations go to my daily supervisor Antonio Jarquin Laguna, for keeping me on track and trusting my work even while I was in doubt during our meetings.

I also want to thank Arjan Gõbel and Frederik Gerner. Without their curiosity and love for new and innovative technologies, I would never have been able to work on this project. Thank you both, for the support you gave me in the past year and for the useful and informative meetings and brainstorm sessions. I was fortunate to meet the driving forces behind 'Neptune wave energy LTD'. My thoughts go to Cris Taylor's family. Chris, it was a pleasure to have met and see the passion and love for the Neptune, I feel honoured of being a part of the Neptune family. David, thank you for the delightful and enjoyable meetups.

I would like to thank my family, 'Huize Hippo' and friends for the support and trust they gave me. Special thanks goes to my parents, for there faith in me during the long years I have been a student. Lastly, I want to express my love and gratitude to Annemarie, who as my girlfriend, roommate, advisor and supporter in one means a great deal to me.

Sjors de Rooij

Delft, July 2019 



\section{Contents}

$\begin{array}{ll}\text { Abstract } & \text { iii }\end{array}$

1 Introduction $\quad 1$

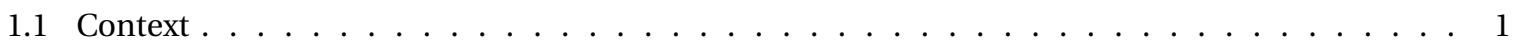

1.1.1 Neptune working principle and background . . . . . . . . . . . . . . . 1

1.2 Problem definition . . . . . . . . . . . . . . . . . . . . . . . 2

1.3 Aim of the research . . . . . . . . . . . . . . . . . . . . . . . 2

1.4 Approach for the research. . . . . . . . . . . . . . . . . . . . 3

1.5 Outline . . . . . . . . . . . . . . . . . . . . . . . . 3

2 Literature Review $\quad 5$

2.1 Energy in waves. . . . . . . . . . . . . . . . . . . . . . . 5

2.1.1 Calculate available power . . . . . . . . . . . . . . . . . . 5

2.2 Wave energy converters. . . . . . . . . . . . . . . . . . . . . 7

2.2 .1 Oscillating water columns . . . . . . . . . . . . . . . . . . . 7

2.2 .2 Oscillating body devices . . . . . . . . . . . . . . . . . . . 7

2.2 .3 Overtopping converters . . . . . . . . . . . . . . . . . . 7

2.3 Numerical Modelling . . . . . . . . . . . . . . . . . . . . . . . . . 8

2.3.1 Oscillating water columns . . . . . . . . . . . . . . . . . . . . . 8

2.3.2 Thermodynamics of an air chamber . . . . . . . . . . . . . . . . 8

2.3 .3 Turbine .............................. 9

2.3 .4 Weir discharge . . . . . . . . . . . . . . . . . . . . . . . 9

3 Theoretical model $\quad 11$

3.1 System description . . . . . . . . . . . . . . . . . . . . . . . 11

3.2 Model Derivation . . . . . . . . . . . . . . . . . . . . . . . . . . . 12

3.2.1 Derivation of the equation of motion water column . . . . . . . . . . . . . . 13

3.2 .2 Weir discharge . . . . . . . . . . . . . . . . . . . . . . . 14

3.2.3 Air pressure ............................ 17

3.2 .4 Hydrodynamic loads. . . . . . . . . . . . . . . . . . . . . . . 18

3.2 .5 Hydraulic turbine . . . . . . . . . . . . . . . . . . . . . . . 22

3.2 .6 Head losses . . . . . . . . . . . . . . . . . . . . . . . . . 24

3.3 Calculation method . . . . . . . . . . . . . . . . . . . . . . . . 24

3.3.1 Eulers method . . . . . . . . . . . . . . . . . . . . . . . . 24

3.3.2 Runge-Kutta methods . . . . . . . . . . . . . . . . . . . 25

3.3.3 Solving the matrix equation . . . . . . . . . . . . . . . . . . . . 26

3.3 .4 Post-processing . . . . . . . . . . . . . . . . . . . . 26

3.4 Conclusion . . . . . . . . . . . . . . . . . . . . . . 27

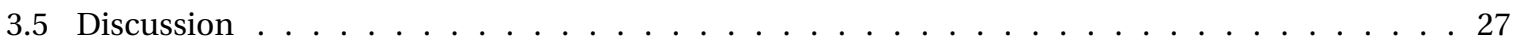

4 Model design $\quad 29$

4.1 Environmental conditions ........................... . . 29

4.1 Site selection. . . . . . . . . . . . . . . . . . . . . . 29

4.1 .2 Wave climate. . . . . . . . . . . . . . . . . . . . . . 29

4.1 .3 Depth ............................... 29

4.2 Linear equations of motion . . . . . . . . . . . . . . . . . . . . . . 30

4.2.1 Equations of motion of the water column . . . . . . . . . . . . . . . . 30

4.2.2 Equations of motion of the air pressure . . . . . . . . . . . . . . . . . 31 


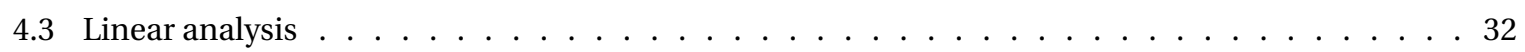

4.3.1 Starting points . . . . . . . . . . . . . . . . . . . . . 32

4.3.2 Frequency domain solution . . . . . . . . . . . . . . . . . . . . 32

4.3 .3 Dimensions . . . . . . . . . . . . . . . . . . . . 35

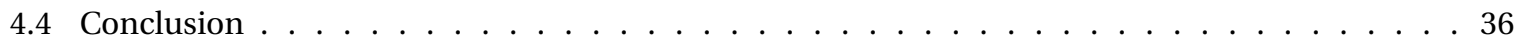

5 Time domain results $\quad 37$

5.1 Wave parameters, forces and hydrodynamic coefficients . . . . . . . . . . . . . . . 37

5.2 Response excluding power take-off . . . . . . . . . . . . . . . . . . . . . 37

5.2.1 Response excluding weir discharge. . . . . . . . . . . . . . . . . . . . . 37

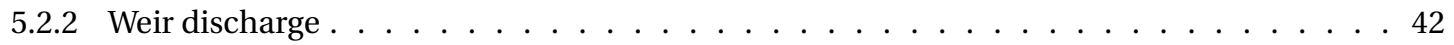

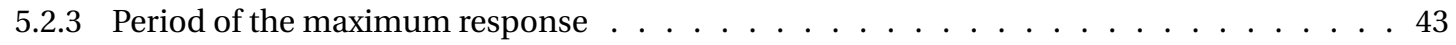

5.3 Response including power take-off . . . . . . . . . . . . . . . . . . . . 46

5.3 .1 Turbine response . . . . . . . . . . . . . . . . . . . . 46

5.3 .2 Turbine diameter . . . . . . . . . . . . . . . . . . . . . . 49

5.3 .3 Wave height . . . . . . . . . . . . . . . . . . . . 51

5.4 Conclusion and Discussion . . . . . . . . . . . . . . . . . . . . . . . 52

5.4.1 Response excluding power take-off . . . . . . . . . . . . . . . . . . . . 52

5.4 .2 Response including power take-off . . . . . . . . . . . . . . . . . . . 54

5.4 .3 Discussion . . . . . . . . . . . . . . . . . . . . 54

6 Comparison scale model $\quad 55$

6.1 Scale model. . . . . . . . . . . . . . . . . . . . . . . . 55

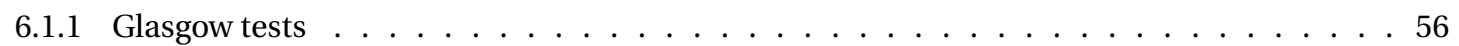

6.1 .2 Plymouth tests. . . . . . . . . . . . . . . . . . . . 56

6.1 .3 Simulated model. . . . . . . . . . . . . . . . . . . . . . 57

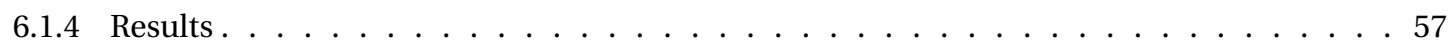

6.2 Conclusion and discussion . . . . . . . . . . . . . . . . . . . . . . . . 62

6.2 .1 Conclusion. . . . . . . . . . . . . . . . . . . . . . . 62

6.2 Discussion . . . . . . . . . . . . . . . . . . . 62

7 Feasability $\quad 63$

7.1 Power available at location . . . . . . . . . . . . . . . . . . . . 63

7.2 Main structure . . . . . . . . . . . . . . . . . . . . . . . 67

7.3 Turbine . . . . . . . . . . . . . . . . . . . . . . 68

7.4 Fabrication, Transportation and Installation . . . . . . . . . . . . . . . . . . . . . 68

7.5 Internal design . . . . . . . . . . . . . . . . . . . . . . . 70

7.6 Comparison to other wave energy converters . . . . . . . . . . . . . . . . . 71

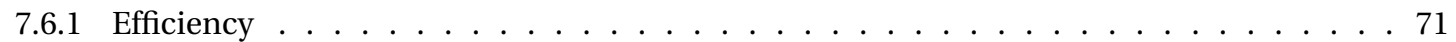

7.6 .2 Geometrical . . . . . . . . . . . . . . . . . . . . 72

7.7 Economic feasibility . . . . . . . . . . . . . . . . . . 73

7.8 Conclusion . . . . . . . . . . . . . . . . . . . . . . 75

7.9 Discussion . . . . . . . . . . . . . . . . . . . . . 75

8 Conclusion and recommendations $\quad 77$

8.1 Conclusion . . . . . . . . . . . . . . . . . . . . . . 77

8.1.1 Dynamic response to periodic loads . . . . . . . . . . . . . . . . . 77

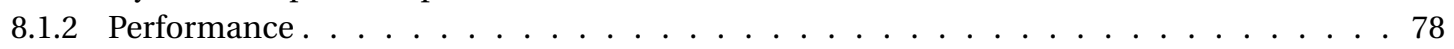

8.1 .3 Validation ......................... . . . . . . . . . . . . . . . . . . . . 78

8.2 Recommendations . . . . . . . . . . . . . . . . . . . . . . 79

$\begin{array}{ll}\text { Bibliography } & 81\end{array}$

$\begin{array}{ll}\text { List of Figures } & 85\end{array}$

$\begin{array}{ll}\text { List of Tables } & 89\end{array}$

$\begin{array}{ll}\text { A Turbine Characteristics } & 91\end{array}$

B Appendix A: Wave data Hebrides Shelf 93 
C Results variable turbine diameter $\quad 97$

C.1 Results weir level 0.5 meter . . . . . . . . . . . . . . . . . . . . . . . . . . . 97

C.1.1 Turbine diameter 1.5 meter . . . . . . . . . . . . . . . . . . . . . 97

C.1.2 Turbine diameter of 1.75 meter . . . . . . . . . . . . . . . . . . . 98

C.1.3 Turbine diameter of 2 meter . . . . . . . . . . . . . . . . . . . . . . 100

C.1.4 Turbine diameter of 2.25 meter . . . . . . . . . . . . . . . . . . . . . . . . 101

C.1.5 Turbine diameter of 2.5 meter . . . . . . . . . . . . . . . . . . . . . . . . 102

C.2 Results weir level 0.7 meter . . . . . . . . . . . . . . . . . . . . . . . . . . . . 104

C.2.1 Turbine diameter of 2 meter . . . . . . . . . . . . . . . . . . . . . . . . . 104

D Glasgow scale tests Neptune wave energy converter 107

D.1 Glasgow scale tests . . . . . . . . . . . . . . . . . . . . . . . . . . . . . 107

D.2 Plymouth test. . . . . . . . . . . . . . . . . . . . . . . 110 



\section{1}

\section{Introduction}

In the past decades, the demand for energy increased significantly. The majority of the energy is currently generated from non-renewable resources. The finite supply and environmental impact have led to an increased demand for energy harvesting technologies from renewable resources. A potential resource is wave energy, with worldwide potential energy resource of 2 Terra Watt (TW), this can provide a significant part of the current global electricity use [1]. The research aims to contribute to the development of renewable wave energy. In this chapter, the context for this research is presented together with the working principle and background of the concept investigated in this report. Additionally, the problem definition is given together with the aim of the research. Finally, the approach and outline of the report are presented.

\subsection{Context}

Over the years many types of wave energy converters (WEC) have been invented and developed, varying from submerged bodies to oscillating water columns (OWC). The concept of an OWC comes down to an air chamber connected to the outside water, the water column within the air chamber oscillates as a response to the incoming waves. Under resonance conditions the motion is amplified forcing a high amount of air in and out the air chamber through a turbine, generating energy. This air flow requires the air chamber to be connected to the atmosphere resulting in a design penetrating the free-surface of the ocean. At the free surface, the structure is subjected to wave impact and wind loads and marine growth. A submerged structure would benefit from reduced environmental loads and a simplified structural design. Considering these benefits a new concept was conceived by D. Carey, a senior engineer with the Vickers Shipbuilding Company, in the mid-1970s [2]. This concept combined the principle of oscillating water columns with a fully submerged structure.

\subsubsection{Neptune working principle and background}

The proposed system consists of a fully submerged structure with two water columns, separated by a weir and an air chamber. The basic principle of the generation of energy is a positive net flow of water through the system as presented in figure 1.1. The pumping motion is obtained by a single water column resonating at a range of wave periods. The amplified motions result in a discharge over the weir into the second column. This column has a small exit pipe leading to a smaller response to the oscillating pressures of the waves and air pressure of the air chamber. The weir discharge combined with the small response to the oscillating results in a uni-directional flow in the second column where an installed turbine can extract energy from this flow.

The concept was pursued and further investigate by Vickers Shipbuilding Company until the discovery of fossil fuels in the North Sea initiating the end of the oil crisis. The project was resurrected in the late 1990s in the form of 'Neptune Energy LTD'. A design was proposed consisting of a 'doughnut-like' structure, with an overall diameter of $40 \mathrm{~m}$ and height of $25 \mathrm{~m}$, housing the two water columns an air chamber, enabling an optimal response for omnidirectional wave directions. The concept was further drawn out and a scale model was built for testing. During the two scale tests, in 2008 in Glasgow and 2013 in Plymouth, the concept of the Neptune was proven using the same $1 / 20^{\text {th }}$ model, figure 1.2 a picture of the Neptune scale model in the test basin in Glasgow. Both tests were performed for similar conditions, unfortunately, both excluding the influence of power take off in the exit duct. After the tests no major actions have been undertaken, and the so 

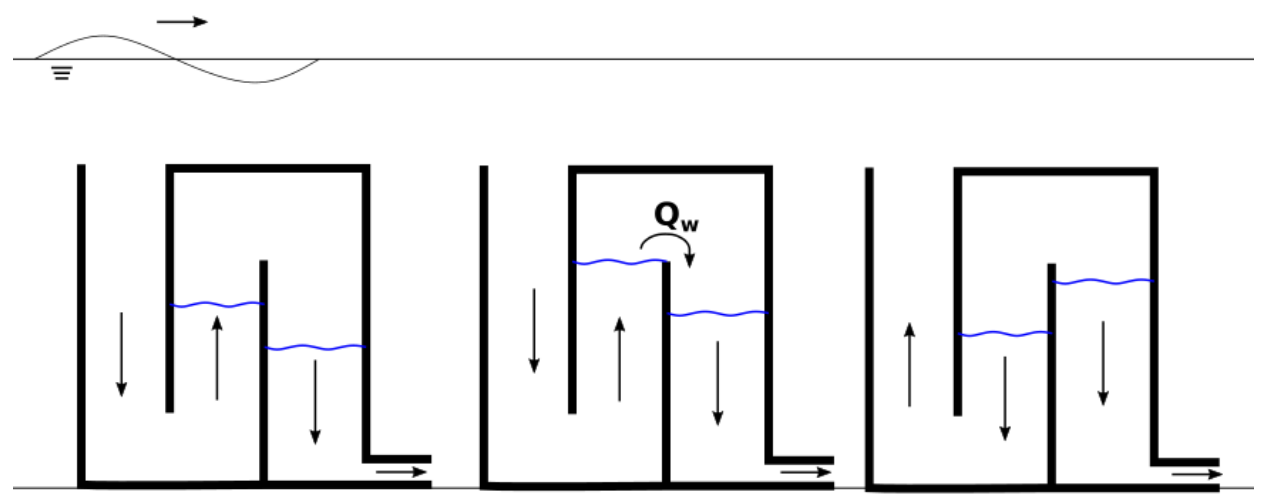

Figure 1.1: Schematic representation of the motion

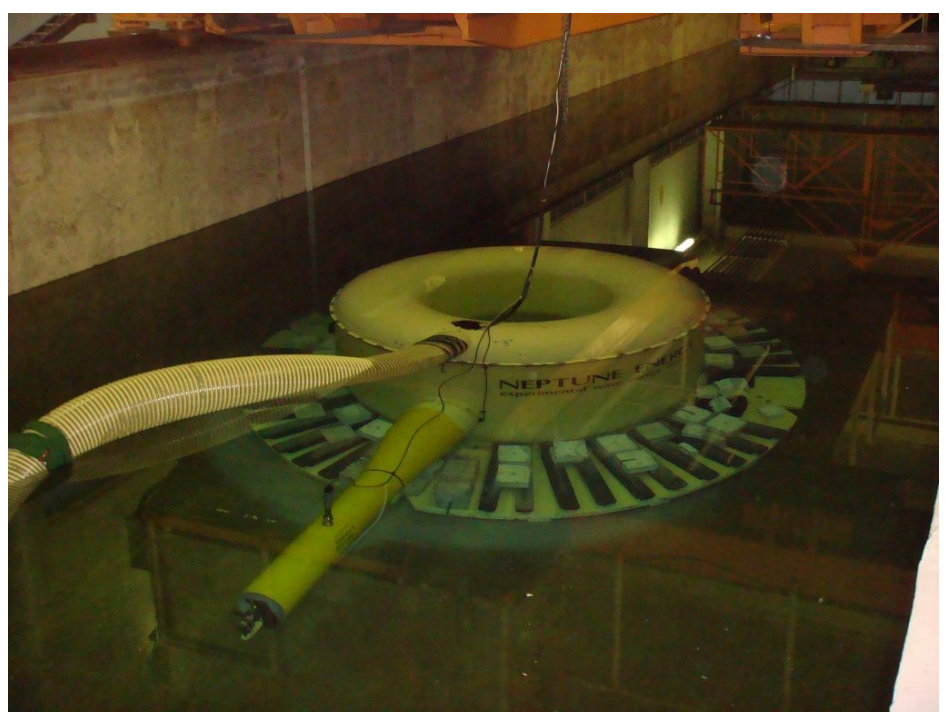

Figure 1.2: Scale prototype Neptune Energy LTD, in testing facilities Glasgow 2008

far obtained knowledge and experiments is the starting point of this research.

\subsection{Problem definition}

As described in the previous section a $1 / 20^{\text {th }}$ was tested. The test proved the concept of the water being pumped through the system by the resonating response of the first column. Additionally, some work was done on the behaviour of a single column with mass transfer [3-5] in addition to the research on the behaviour of conventional oscillating water column [6,7]. However, the coupled system of two submerged columns has not been investigated in detail, known to the author. Secondly, the scale test did not incorporate the damping associated with the generation of energy by a turbine. Concluded, a working concept has been developed but is lacking a scientific basis describing the full dynamics of the energy converting system.

\subsection{Aim of the research}

The aim of this research is to investigate the behaviour of the presented system. The analysis of the behaviour can be split into two parts. The first part is to investigate the effect of the coupling of the two columns by the air chamber in addition to the mass transfer. The second part is to analyse the effect of the power take-off on the response to predict the performance of the Neptune. To achieve the aim of this research the following main and sub-questions will be answered at the end of this report.

- How does the model respond to a periodic load? 
- What is the influence of the mass transfer between the two columns?

- What is the influence of the power take-off?

- What is the performance of the model?

- What is the influence of the turbine diameter?

- What is the influence of the incoming wave height?

- How does the performance compare to other WECs?

- How does the numerical model compare to the performed experiments?

\subsection{Approach for the research}

To reach the aim of this research a model is made to simulate the behaviour of the system. Firstly, a set of non-linear differential equations is derived for the motion of the water columns and the dynamics of the air chamber. Additional equations and variables are derived representing the hydrodynamic loads, the response of the turbine, the mass transfer between the columns and additional changes in momentum associated with the mass transfer. It is important to note that the determination of the hydrodynamic coefficients and the design of a variable speed turbine do not fall within the scope of this research. Secondly, the non-linear equations have been linearized to determine the full-scale dimensions for the desired response of the system. Next, the non-linear response is solved by numerical integration in the time domain for regular waves only. Furthermore, the dimensions of the scale model of the experiments represented for the numerical model and its results compared with each other. Finally, the technical, operational and financial feasibility will be assessed.

\subsection{Outline}

In the second chapter, the literature study is presented, discussing the state of the art of WEC technology. In chapter 3 the theoretical model is derived. The starting points and the requirement for the model are determined in chapter 4 together with the full-scale dimensions used to obtain the time domain results of the system in chapter 5. In this chapter, the influence of different parameters is presented together with the performance of the system. In the sixth chapter, the previously performed scale tests are presented and compared to the numerical model. In chapter 7 the technical, operational and commercial feasibility of the proposed design is presented. The final chapter will answer the research questions stated in section 1.3 together with the recommendations for further research. 



\section{Literature Review}

In this chapter, the state of the art of wave energy technology is introduced. First, the potential of wave energy is described. Then, various concepts of wave energy converters are presented and followed by the associated modelling principles of the considered concept.

\subsection{Energy in waves}

First, it is essential to analyse the potential power available in the waves. As the wind blows over the water, it transfers energy to the waves. This provides an energy concentration in the water near the free surface. The energy density of sea waves is high compared to other renewable energy sources [8]. The waves can travel thousands of kilometres, from the American side of the Atlantic Ocean to Europe, with little energy loss. Travelling east with a westerly wind, the energy flux in those incoming waves can be considerable in deep waters. While approaching the shoreline, the energy decreases due to the interaction with the seabed. Despite the reduced energy in the coastal areas, it has the advantage of being close to the land and power grids. Figure 2.1 depicts the average annual wave power for various locations over the world. This data was used in $[1,9]$ to estimate the theoretical yearly wave power in nearshore areas, suitable for energy converter device. The resource potential is assessed between the 2 and 3 TW. In the following sections, the wave power calculations are presented for regular waves and wave spectra.

\subsubsection{Calculate available power}

Wave measurements detect the surface elevations from which the wave height and periods can be found. From this data, the power in waves can be determined as described by [11]. The first step is to determine the deep water wavelength from the found wave periods:

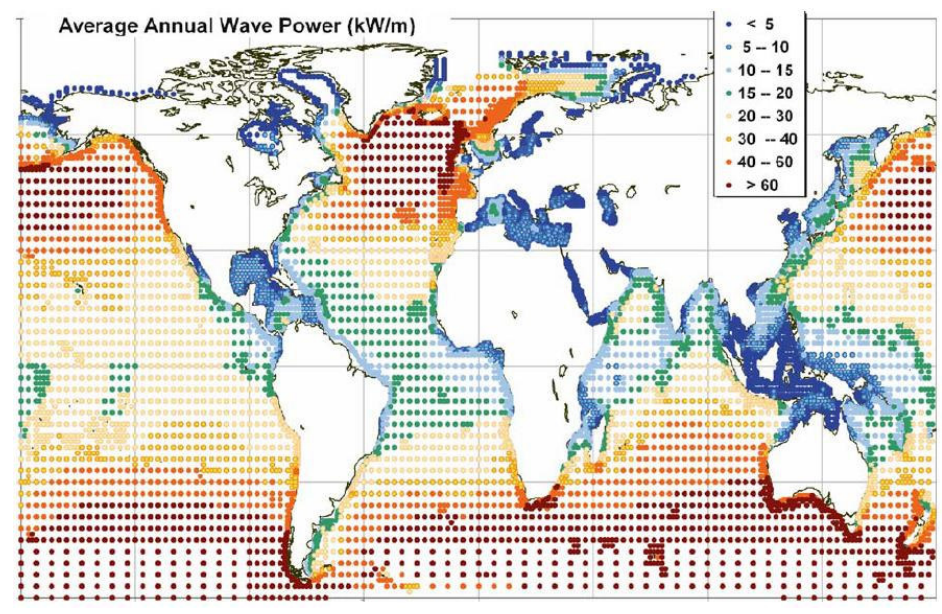

Figure 2.1: Average annual wave power [10] 


$$
L_{0}=\frac{g T_{w}^{2}}{2 \pi}
$$

Where $g=9.81 \frac{\mathrm{m}}{\mathrm{s}^{2}}$ is the gravitational acceleration, and $T_{w}$ is the wave period in seconds. From the deep water wave length the local wavelength is given by:

$$
L=L_{0} \tanh \left(\frac{2 \pi d_{w}}{L}\right)
$$

Where $d_{w}$ is the water dept. Next, the potential, kinetic and total wave energy per unit horizontal area is calculated:

$$
\begin{gathered}
E_{\text {potential }}=E_{\text {kinetic }}=\frac{1}{4} \rho g a_{w}^{2} \\
E_{\text {total }}=E_{\text {potential }}+E_{\text {kinetic }}=\frac{1}{2} \rho g a_{w}^{2}=\frac{1}{8} \rho g H_{w}^{2}
\end{gathered}
$$

Where $\rho \approx 1025 \frac{\mathrm{kg}}{\mathrm{m}^{3}}$ is the density of the seawater, $a_{w}$ the wave amplitude and $H_{w}=2 a$ the wave height in meters. From the energy in waves, the total energy transport, available wave power, is determined:

$$
P_{\text {wave }}=E_{\text {total }} c_{g}
$$

Here $c_{g}$ is the group velocity given by:

$$
c_{g}=n c=\frac{1}{2}\left(1+\frac{2 k_{w} d_{w}}{\sinh 2 k_{w} d_{w}}\right) \sqrt{\frac{g}{k_{w}} \tanh k_{w} d_{w}}
$$

With $k_{w}=\frac{2 \pi}{L}$ being the wave number. The total power per meter wave crest is calculated by:

$$
P_{\text {wave }}=\left(\frac{1}{8} \rho g H_{w}^{2}\right)\left(\frac{1}{2}\left(1+\frac{2 k_{w} d_{w}}{\sinh 2 k_{w} d_{w}}\right)\right) \sqrt{\frac{g}{k_{w}} \tanh k_{w} d_{w}}
$$

For deep water waves, the dispersion relation applies $\omega_{w}=\sqrt{g k_{w}}$ for the linear wave theory and $T_{w}=\frac{2 \pi}{\omega_{w}}$, the power in deep water waves can be approximated by:

$$
P_{\text {wave }} \approx \frac{1}{32 \pi} \rho g^{2} H_{w}^{2} T_{w}
$$

Equation (2.6) is dependent on both the water depth and wavelength. For the relation between the water depth and the wavelength, the water depth conditions are formulated:

- Shallow water when $\frac{d_{w}}{L}<\frac{1}{25}$

- Deep water when $\frac{d_{w}}{L}>\frac{1}{2}$

- Transitional water when $\frac{1}{25}<\frac{d_{w}}{L}<\frac{1}{2}$

The relation between the wave height and the wavelength determines the wave steepness $s=\frac{H_{w}}{L}$. Both the wave steepness and the water depth situations are important when applying the linear wave theory. The linear wave theory can be applied for waves with low steepness and when not in shallow water. A more realistic wave climate approximated by linear wave theory is obtained by the use of a combination of multiple regular waves with different period and amplitudes. However, sea waves are often asymmetric and appear in a wide spectrum of periods and corresponding wave heights. Higher order wave theories are required to allow for such waves. These theories also allow for waves with high steepness and shallow waters. Examples of higher order theories are Stokes wave theory, cnoidal wave theory and stream-function theory. In deep water sea states, the average energy density of the gravity waves is given by:

$$
E_{\text {spectrum }}=\frac{1}{16} \rho g H_{m 0}^{2}
$$

Where $H_{m 0}$ is the significant wave height, derived from the spectral moment. Using the relation of $P=E c_{g}$ results in the average power of the wave spectrum:

$$
P_{i r r}=\frac{1}{64 \pi} \rho g^{2} H_{m 0}^{2} T_{-10}
$$


Where $T_{-10}$ is the spectral peak period of the spectrum. In equations (2.7) and (2.9) it can be seen the total energy in a wave spectrum from the significant wave height and the spectral peak period is half the energy of a single wave with the same wave height and period.

\subsection{Wave energy converters}

Over the years, significant efforts have been made in developing devices to harvest the potential power in the waves. By 1980 already over one thousand patents have been registered with its oldest record dating back to 1799 in France [12]. Even now, new concepts are under research and development. Opposite to the wind industry, where the industry has converged to a general idea, the concepts in the wave energy industry differ significantly from each other. The structures are varying from fixed to floating to wholly submerged. Numerous types of energy converters are used to generate the power; air turbines, hydraulic turbines, hydraulic motors or linear electrical generators. In [12], different types of wave energy converters are conveniently classified by their physical principle in three categories; oscillating water columns, oscillating bodies and over-topping converters. In the following sections, the technology behind these categories is introduced.

\subsubsection{Oscillating water columns}

Oscillating water columns (OWC) generally comprises of a semi-submerged chamber, with an opening below the water surface and air trapped in its upper part. The water column reacts to the periodic pressure fluctuations of the incoming waves, resulting in an oscillating motion of the inner free surface. This motion compresses and decompresses the air in the chamber, forcing the air through a turbine that powers a generator. As a result of the invention of a bi-lateral turbine, such as the Wells turbine invented in the mid-1970s, flow rectifying valves were not required anymore improving the efficiency of the power extraction significantly. The structural designs for OWC are varying between both fixed as floating. Most fixed OWC are installed on the shoreline or near shore since 1985 several full-scale prototypes have been built [7]. The full-scale plant built in 1999 on the island of Pico, Portugal, with a capacity rated at $400 \mathrm{~kW}$ is has been operational until 2018 when it partially collapsed. The low durability is a common issue with the designs of OWCs, the rough environment taking its toll on the structure resulting in high building coast. Some of the more recent designs, therefore, combine the OWC in breakwaters [13].

Apart from a fixed structure, a variety of floating OWCs have been developed. Floating OWCs have the advantage of being able to deploy further away from the shoreline. The first floating wave energy device was a navigation buoy, powered by an air turbine. This device, developed by Yoshi Masuda, was commercialised and deployed since 1965 and is considered the first wave energy device in the modern wave power technology. Later large scale floating devices were developed on large barges or more straightforward concepts of an axisymmetric buoy.

\subsubsection{Oscillating body devices}

In oscillating body devices, energy is generated from the motion of a rigid body excited by wave-induced forces. The motion of the body relative to a reference object is damped extraction power. Over the year many different combinations of shapes and modes of motions between the oscillating body and the reference object are opted, from heaving systems to devices conversing pitch motions into energy. The systems vary from floating to fully submerged structure. An interesting example is the Archimedes Wave Swing [14]; this device is a completely submerged point absorber with an enclosed air chamber, similar to the considered concept. With a bottom fixed air filled silo and a movable upper floater. The floater oscillates due to the wave presses, compressing the air inside. A linear electric generator converts the motion of the floater into electricity.

\subsubsection{Overtopping converters}

The third classification is the overtopping converter. This system forces water over its structure into a reservoir; the elevated water level within the reservoir creates a pressure difference between the reservoir and the sea. By releasing the water back to the sea through turbines, power is generated. This principle is both applied to an onshore reservoir as a floating system. Of the first, a prototype was built in Norway in 1985 in the form of the TAPCHAN (Tapered Channel Wave Power Device). This device had a capacity of $350 \mathrm{~kW}$ and was operated for a few years [12]. A more recent device is the Wave Dragon [15], this floating device reservoir with wings to guide the waves to the centre ramp and into the reservoir. 


\subsection{Numerical Modelling}

This section aims to give insight into the theoretical and numerical modelling of wave energy devices, focusing on the aspects related to the modelling of the Neptune. This comprises the motion of an oscillating water column, the pneumatic air conditions, weir overflow and turbine modelling.

\subsubsection{Oscillating water columns}

This section focuses on the theoretical hydrodynamic modelling of OWCs. The first theoretical model was described by McCormick [16, 17]. Here, followed by Evans [18], the equation of motions were derived assuming no horizontal variations in the elevation of the air chamber, the rigid piston approach. This approach models the internal water surface as a rigid piston, where the volume flow is directly related to the displacement of the water surface $\mathrm{x}$. The governing equation was given by:

$$
m \ddot{x}=-\rho g A_{c} x+f_{r}+f_{e}-A_{c} p
$$

Here $m$ is the mass of the piston, $A_{c}$ is the area of the piston, $\rho$ is the water density, $g$ is the acceleration of gravity, $f_{r}$ is the hydrodynamic radiation force, $f_{e}$ is the excitation force, and $p$ is the excess pressure of the air chamber. In reality, the surface elevation varies over its width and does not satisfy the dynamic boundary condition of the linearised hydrodynamic theory for irrotational flow [19]. The correct way of modelling is the applied-pressure description, which complies with the dynamic boundary condition. In the latter method, the volume flow is derived from the change of air volume in the chamber. Decomposing the flow rate of the column into a radiation flow rate, due to the motions in absence of the waves, and the excitation flow rate, due to the incoming waves in the absence of a dynamic pressure in the chamber, allows us to solve the problem as a linear wave diffraction problem common in ship hydromechanics [20]. The rigid piston gives a good approximation for low frequencies, and a very long wavelength compare to the horizontal length of the system.

The pressure in the air chamber is a function of the volume of air and the thermodynamics of the (de)compression process in the air chamber. In a simplified situation, we would assume a linear relationship between the pressure and displacement of the piston or volume flow. It is resulting in a system that can be linearised and solved in a frequency domain analysis. However, the thermodynamic process is not linear and requires the system to be solved in a time domain analysis. Furthermore, in equation (2.10), the radiation and excitation forces are not specified. The radiation force can be decomposed in the frequency dependent the added mass and the radiation damping. In the frequency domain analysis, these coefficients can be solved for each specific frequency. A common representation has been given by Cummings to apply these coefficients in the time domain. Assuming the added mass to for a very high frequency, $m_{a, \infty}$ and expressing the radiation damping by a convolution integral, this gives:

$$
\left(m+m_{a, \infty}\right) \ddot{x}-\int_{0}^{t} H(t-\tau) \dot{x} d \tau+\rho g A_{c} x+A_{c} p=f_{e}
$$

Where $\mathrm{H}$ is the memory function related to the radiation damping, to solve the system, the hydrodynamic coefficients still have to be computed. Codes based on the boundary element method can compute the coefficients for ships and marine structures. Lighthill [3] derived the hydrodynamic coefficients in a twodimensional mathematical analysis for submerged resonant ducts. The coefficients are related to the wavelength geometry of the entrance, the direction of the opening concerning the free surface, the width and the submerged depth. In this model, a rigid body approach was used. The models above are based on Laplace's equation and linear wave theory, to account for fluid dissipation effects coefficients have been derived from experiments to be used in the simplified models [21-23]. Such effects may be over or underestimated in the high energy conditions close to resonance. To account for these effects, computational fluid dynamics may be deployed, based on the Reynold-averaged Navier-Stokes (RANS) equation in combination with an appropriate turbulence model:

\subsubsection{Thermodynamics of an air chamber}

As mentioned in the section above, the compression and decompression of air is a non-linear process. This effect was modelled for the first time in ref [24] as a simple isentropic relationship between air pressure and the density, assuming adiabatic conditions, given the small temperature oscillation and short timescale for heat exchange. However, the turbine flow changes the entropy of the system, mainly during the inhalation 
phase [25]. During the exhalation phase, constant entropy for the air remaining in the chamber was found. Consequently, for isentropic processes, we can write:

$$
\left(\rho_{\text {air }}\left(p_{a}+p\right)^{-\frac{1}{\gamma}}=\rho(t)\left(p_{a}+p+p(t)\right)^{-\frac{1}{\gamma}}\right.
$$

Where $\rho_{\text {air }}$ and $\rho(t)$ are the densities of the respectively uncompressed air and time-dependent compresses air, $p_{a}, p$ and $p(t)$ are respectively the atmospheric, static and time-dependent pressure in the chamber and $\gamma$ denotes the polytropic exponent. For a perfect turbine resulting in an isentropic pressure condition with $\gamma=1.4$. In the Archimedes Swing, containing a fully enclosed air chamber, the relation is used to derive the pressure due to the volume change: [26]

$$
p_{a}=p_{a, a v}\left(\frac{V_{a c t, a v}}{\left(V_{a c t}(t)\right)}\right)^{\gamma}
$$

Where $V_{a c t, a v}$ and $p_{a, a v}$ is the time-averaged volume and air pressure in the chamber and $V_{a c t}(t)$ the time-dependent instantaneous volume.

\subsubsection{Turbine}

As mentioned in the general section of OWCs, the air turbine response can be estimated for a linear relationship between the mass flow and pressure from the turbine characteristic curves. The pressure and direction of the flow oscillate with the motion of the free surface, to take this into account the bi-axial turbines, such as the Well turbines, are used.

In hydro plants hydraulic turbines are used, the energy generation is power by the pressure and discharge relation.

$$
P=Q \Delta p
$$

Here $P$ is power in Watt, $Q$ is the discharge in cubic meter per second and $\Delta p$ the pressure difference between both sides of the turbine in Pascal. The actual power generated also depend on the dimensions and rate rotation speed of the turbine. The generated power is derived from regression relations between all variables [27]. For most hydro energy plants, the pressure and discharge are stationary. The energy extraction can, therefore, be optimised easily for the given pressure head. The extraction of energy in less stationary flows introduces some difficulties.

For example, the Wave Dragon is required to operate at discharges, and pressure heads varied over time [15]. A Kaplan turbine was designed to account for these variations. Consisting of a fixed number of blades and fixed guide vanes. Many smaller turbines are used to improve the efficiency of the operation, which can be switched on and of separately. For optimal performance in a wide range of pressure heads, $0.4-4 \mathrm{~m}$, the turbines were designed to operate at variable speed, using inverter controlled permanent magnet generators. Variable speeds direct drive power generators are also found in the wind industry, using a frequency converter allows for the variable speed to adapt for the variable wind speeds $[28,29]$.

\subsubsection{Weir discharge}

Lastly, the modelling of the weir discharge is discussed. The flow over a weir is widely discussed and analysed for mainly horizontal steady flow conditions. In these conditions, the discharge over the weir can be expressed as a function of the height of the free surface above the weir $H$, the width of the weir $b$ and the dimensionless geometrical parameter $C$, representing ration of the actual and the theoretical discharge determined experimentally. [30]

$$
Q=g^{\frac{1}{2}} H^{\frac{3}{2}} b C
$$

In [31] and experiment was described consisting of a vertical duct out from which water was pumped. The top of the bulge concerning the exit was measured for different velocities resulting in the following relations:

$$
h=\sqrt[3]{\frac{D V^{4}}{g^{2}}}
$$

This relation was obtained for a cylindrical duct for free flow in all directions. In [5], the dynamics response of a similar system was analysed numerically, the fluid above the exit assumed to be drawn off instantaneously for the complicity of the dynamic flow. 



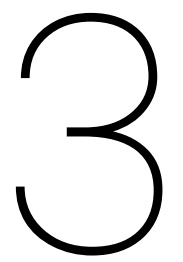

\section{Theoretical model}

The present chapter describes the modelling approach toward the physics around the Neptune wave energy converter. First, the geometrical scheme of the concept is presented, followed by the derivation and description of the non-linear governing equations motion for the water columns and pressure in the air chamber. From the non-linear simplified linear equations will be obtained.

\subsection{System description}

The Neptune Energy LTD wave energy converter concept design is depicted in figure 3.1. This circular concept consists of four key elements, the central water column with a vertical entrance to the surrounding water, the inner air chamber including the separation weir, the second water column and the turbine in the exit pipe to the surrounding water. For the purpose of the analysis a rectangular concept is proposed, presented in figure 3.2 .

The dimensions and parameters in 3.2 represent the initial volume $V_{0}$ and the initial pressure $p_{0}$ of the air chamber, the pressure is given by:

$$
p_{0}=p_{a t m}+\rho g h_{c}+p_{a i r}(t=0)
$$

where $p_{a t m}$ is the atmospheric pressure, $h_{c}$ is the equilibrium level of the inner free surfaces of the column with respect to the surface level of the surrounding water and $p_{\text {air }}$ the time dependent variable of the pressure difference inside the chamber. The two water columns are denoted by the subscripts 1 and 2 , for respectively the left and the right column. The columns are separated by the air chamber and the weir, the level of the weir is denoted by $z_{w}$ positive upwards from $h_{c}$. The free-surface levels of both columns are given by the time dependent variable $x_{1}$ and $x_{2}$, positive values are upward toward the free surface of the surrounding water and zero at $h_{c}$. The area $A$ in the columns is composed of the width of the column and the total width of the structure, in-plane in figure 3.2. The areas are assumed constant over the lengths $L$ of the sections, where

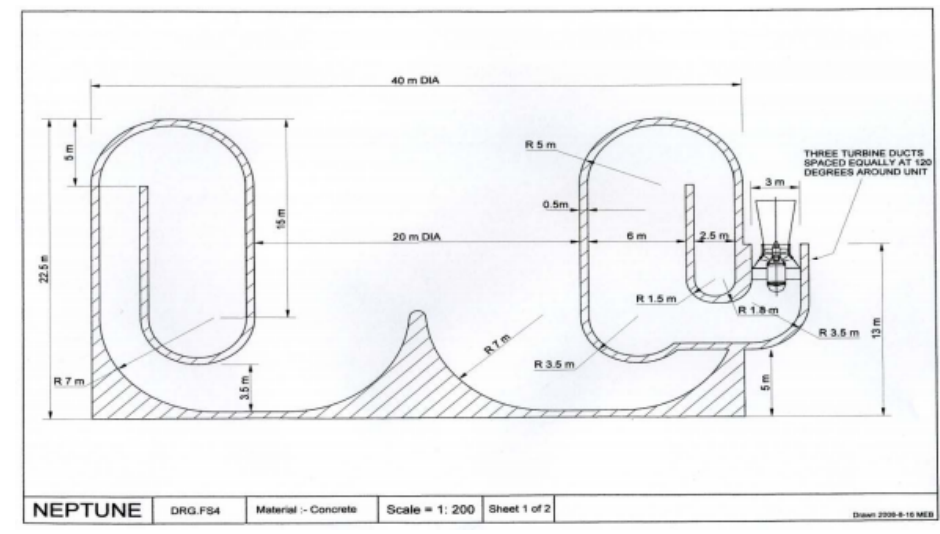

Figure 3.1: Drawing full scale Neptune, as design of Neptune Energy LTD. 


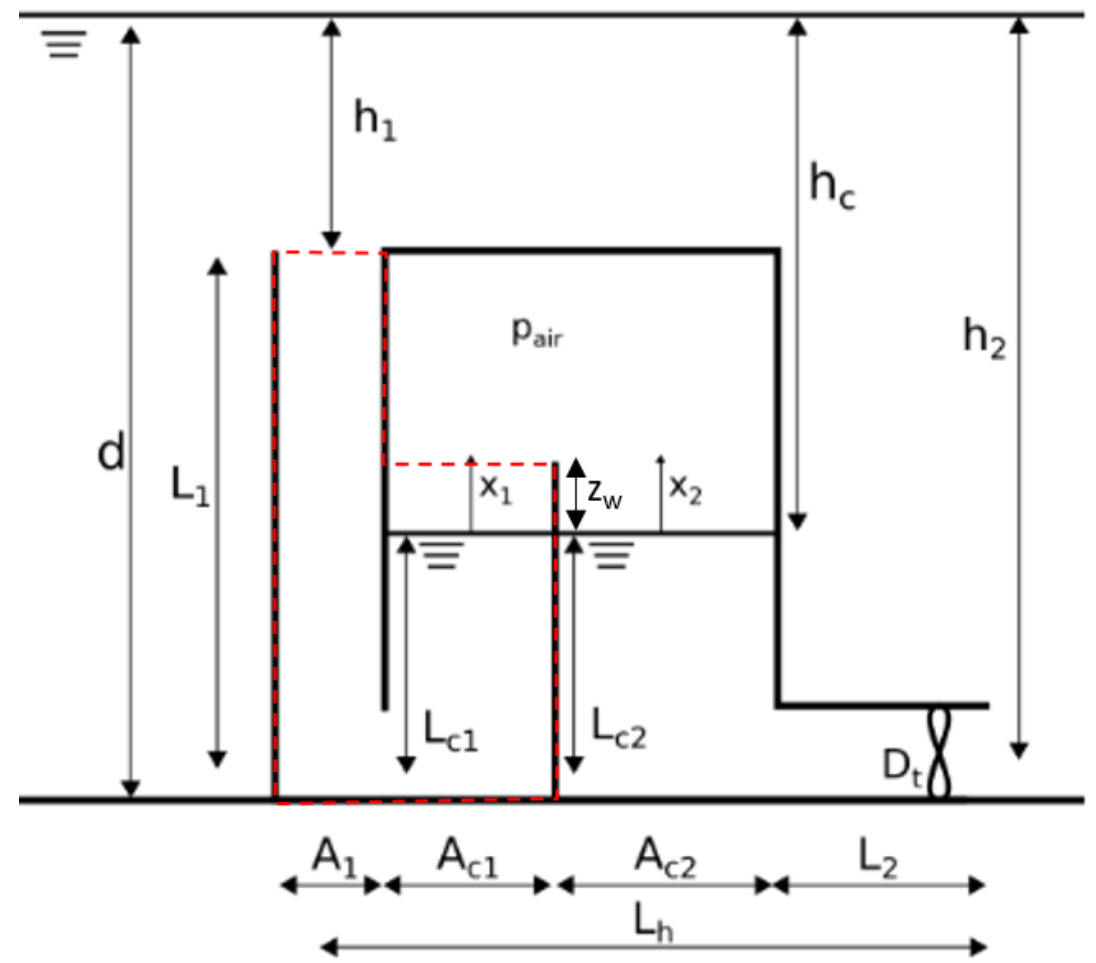

Figure 3.2: Cross-section of the submerged Oscillating water column, symbols denote the geometrical characteristics.

the subscripts $\mathrm{c} 1$ and $\mathrm{c} 2$ denote the vertical sections below the inner free surface and 1 and 2 the sections before the openings to the surrounding water. To incorporate an turbine in the column section $L_{2}$, it has a circular cross-sectional area $A_{2}$, with diameter $D_{t}$, much smaller than the areas $A_{1}, A_{c 1}$ and $A_{c 2}$, as discussed in chapter 1 .

\subsection{Model Derivation}

In the previous section the time dependent variables for the inner free-surface displacements were defined, the free surfaces are connected to the surrounding waters. The flow in the fluid medium is expressed in terms velocity potentials $\phi$, under assumptions of linear water-wave theory. The potentials needs to satisfy

$$
\begin{array}{cc}
\nabla^{2} \phi=0 & \text { in fluid domain } \\
\mathbf{n} \cdot \nabla \phi=0 & \text { on fixed boundaries }
\end{array}
$$

Where $\mathbf{n}$ is the normal vector at the boundary. The linearized dynamic free surface conditions of the free-surfaces $\eta$ are given by:

$$
-\frac{\partial \phi}{\partial t}+g \eta= \begin{cases}\frac{p_{a i r}}{\rho_{w}} & \text { on internal free surface } \\ 0 & \text { on outer free surface }\end{cases}
$$

The kinematic free surface conditions are given by:

$$
\frac{\partial \eta}{\partial t}=\frac{\partial \phi}{\partial z}
$$

As discussed in the previous chapter, the variation of the inner free-surface over its width can be solved by several approaches. Under the assumptions of a long wave length $L_{w}$ compares to the width of the column, neglecting the inner free surface variation results in a good approximation of the real situations. Using this rigid piston approach to model the inner free-surfaces the velocity of the free surface is directly related to the free surface, resulting in the expressions of the potentials:

$$
\frac{\partial \phi_{1,2}}{\partial x}=\dot{x}_{1,2}
$$


Additionally, assuming an incompressible fluid and neglecting the velocity profile within in the crosssection, the velocities inside the columns sections can be written by:

$$
\begin{gathered}
v_{1}=\frac{A_{c 1}}{A_{1}} \dot{x}_{1} \\
v_{c 1}=\frac{A_{c 1}}{A_{c 1}} \dot{x}_{1} \\
v_{2}=\frac{A_{c 2}}{A_{2}} \dot{x}_{1} \\
v_{c 1}=\frac{A_{c 2}}{A_{c 2}} \dot{x}_{1}
\end{gathered}
$$

Under the assumptions above the column can be seen as a rigid oscillating body. In the following section the equations of motions for the system are derived.

\subsubsection{Derivation of the equation of motion water column}

In this section the equation a motion an oscillating water column is derived from the for the control volume $V_{c}$, indicated in figure 3.2 by the red box. The volume occupies the full volume from the entrance to the weir level.

$$
V_{c}=L_{1} A_{1}+\left(L_{c 1}+z_{w}\right) A_{c 1}
$$

Within the control volume the total mass is changes with the motions of the free surface. To account for the internal change of mass the equations are derived from the $1 \mathrm{D}$ conservation of mass and momentum equations. This approach differs from previous works which derive the motions of water columns [5, 13, 31, 32] from the Bernoulli equation. However, the Bernoulli equation does not take into account the change of mass.

\section{Conservation of momentum}

The conservation of momentum equation is given by [33]:

$$
\int_{V_{c}} \frac{\partial \rho \mathbf{v}}{\partial t}+\nabla \cdot(\rho \mathbf{v v}) d V_{c}=\int_{S}-p \mathbf{n} d S+\int_{V_{c}} \rho \mathbf{g} d V_{c}=\int_{V_{c}}-\nabla p+\rho \mathbf{g} d V_{c}
$$

This equation can be simplified by removing the integrands over the same control volume, resulting in:

$$
\frac{\partial \rho \mathbf{v}}{\partial t}+\nabla \cdot(\rho \mathbf{v v})=-\nabla p+\rho \mathbf{g}
$$

This can be written as:

$$
\nabla \cdot(\rho \mathbf{v v})=[\nabla \cdot(\rho \mathbf{v})] \mathbf{v}+\rho \mathbf{v} \cdot \nabla \mathbf{v}
$$

Applying the chain rule differentiation, the momentum equation can be written as:

$$
\left[\frac{\partial \rho}{\partial t}+\nabla \cdot(\rho \mathbf{v})\right] \mathbf{v}+\rho\left[\frac{\partial \mathbf{v}}{\partial t}+\mathbf{v} \cdot \nabla \mathbf{v}\right]=-\nabla p+\rho \mathbf{g}
$$

From the equation the momentum associated to the velocity and accelerations can be recognised in respectively the first and second term on the LHS.

\section{Conservation of mass}

The term for the velocity momentum can be derived from the conservation of mass of the Reynolds transport theorem [34]. Assuming no weir discharge in this derivation the change in mass equals the mass inflow at the entrance:

$$
\frac{d m}{d t}=\int_{V_{c}} \frac{\partial \rho}{\partial t} d V+\int_{V_{c}} \nabla \cdot(\rho \mathbf{v}) d V_{c}=\rho A_{c 1} \dot{x}_{1}
$$

Solving the integrands for control volume $V_{c}$ :

$$
V_{c}\left[\frac{\partial \rho}{\partial t}+\nabla \cdot(\rho \mathbf{v})\right]=\rho A_{c 1} \dot{x}_{1}
$$

Here the first term of 3.11 is recognised. 


\section{Acceleration term}

The second term in (3.11) is the expression for the acceleration of the fluid particles. Assuming an inviscid, irrotational and incompressible fluid this term can be written in the expressions containing the local acceleration and the convective acceleration.

$$
\rho\left[\frac{\partial \mathbf{v}}{\partial t}+\mathbf{v} \cdot \nabla \mathbf{v}\right]=\rho \frac{\partial \mathbf{v}}{\partial t}+\rho \nabla \frac{1}{2} \mathbf{v}^{2}
$$

\section{Equation of motion}

Substituting equations 3.13 and 3.14 in (3.11) gives.

$$
\rho \frac{\partial \mathbf{v}}{\partial t}+\rho \nabla \frac{1}{2} \mathbf{v}^{2}+\rho \frac{A_{c 1} \dot{x}_{1}}{V_{c}} \mathbf{v}=-\nabla p-\rho g
$$

Integrating the particle acceleration over the stream line of the column results in:

$$
\int_{S_{c}} \frac{\partial \mathbf{v}}{\partial t} d S_{c}=\left(\frac{A_{c 1}}{A_{1}} L_{1}+L_{c 1}+x_{1}\right) \ddot{x}_{1}
$$

The change of the convective acceleration is only present at the free surface resulting in the integration of:

$$
\int_{S_{c}} \nabla \frac{1}{2} \mathbf{v}^{2} d S_{c}=\frac{1}{2} \rho \dot{x}_{1}^{2}
$$

Assuming the rigid body translation, the only change in momentum occurs at the free surface, as seen in the convective accelerations. The integration of $\mathbf{v}$ becomes.

$$
\int_{S_{c}} \mathbf{v} d S_{c}=x_{1} \dot{x}_{1}
$$

Resulting in the full expression for the mass momentum off:

$$
\int_{S_{c}} \rho \frac{A_{c 1} \dot{x}_{1}}{V_{c}} \mathbf{v} d S_{c}=\frac{x_{1}}{V_{c}} A_{c 1} \rho \dot{x}_{1}^{2}
$$

Lastly the integration of the RHS gives:

$$
\int_{S_{c}}-\nabla p-\rho g d S_{c}=p+\rho g x_{1}
$$

Resulting in the equation of motion of the water column:

$$
\rho\left(\frac{A_{c 1}}{A_{1}} L_{1}+L_{c 1}+x_{1}\right) \ddot{x}_{1}+\left(\frac{A_{c 1} x_{1}}{V_{c}}+\frac{1}{2}\right) \rho \dot{x}_{1}^{2}=p-\rho g x_{1}
$$

Where $p$ represents the external pressures on the boundaries.

\subsubsection{Weir discharge}

Equation (3.21) describes the motion of the column for displacements, in general for values of $x_{1,2}<z_{w}$ the weir discharge equals zero resulting in the mass change to equal inflow of volume at the opening to the surrounding water. For values of $x_{1,2} \geq z_{w}$ however this relation does not hold and the weir discharge $Q_{w}$ has to be taken into account.

In this analysis an instantaneous weir discharge is assumed, the expression for the weir discharge now becomes. Taking into account the instantaneous weir discharge, it can be described as a function of the column velocities and areas, for the conditions presented in figure 3.3. In (a) the upward going free surface of the resonating first column reaches the weir level and the discharge to the second column equals the total volume flow entering the column at the mouth. In (b) the level of the second column has increased caused by the weir flow and both levels are assumed to rise of drop at the same rate, dependent on the volume flow of both columns. (c) describes the opposite condition of (a), this conditions is unlikely to occur where the flow direction of the second column is design to flow out of the system. Lastly (d) described the motion of the column without the free surface levels at or above the weir, here both column are only coupled by the 
air pressure. As presented in the figure the weir discharge is assumed positive from column 1 to column 2 , resulting in the following equation for the weir discharge.

$$
Q_{w}= \begin{cases}\dot{x}_{1} A_{c 1} & x_{1} \geq z_{w}, x_{2}<z_{w}, \dot{x}_{1}>0 \\ \left(\dot{x}_{1}-\frac{\dot{x}_{1} A_{c 1}+\dot{x}_{2} A_{c 2}}{A_{c 1}+A_{c 2}}\right) A_{c 1} & x_{1} \geq z_{w}, x_{2} \geq z_{w} \\ -\dot{x}_{2} A_{c 2} & x_{1}<z_{w}, x_{2} \geq z_{w}, \dot{x}_{2}>0 \\ 0 & x_{1}<z_{w}, x_{2}<z_{w}\end{cases}
$$

(a)

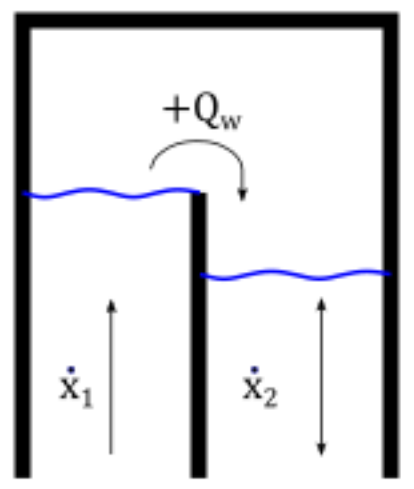

(c)

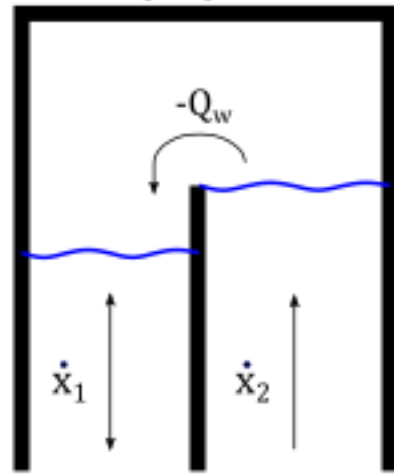

(b)

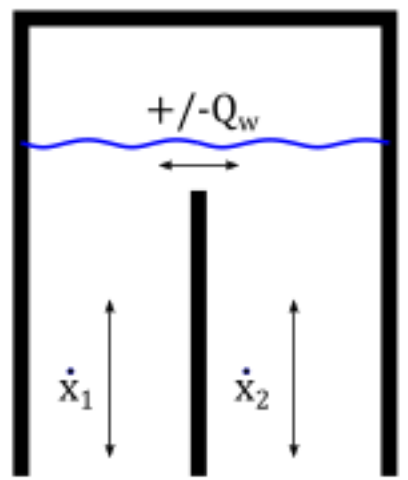

(d)

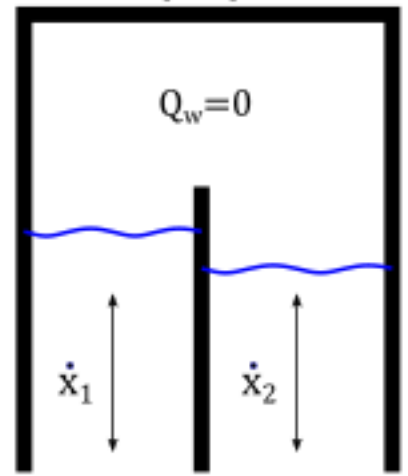

Figure 3.3: Weir flow conditions

During weir discharge the change of volume inside the column is not only related to the volume flowing into the column at its mouth. Now this volume change is also dependent on the weir discharge.

$$
\begin{aligned}
& \frac{d V_{1}}{d t}=\dot{x}_{1} A_{c 1}-Q_{w} \\
& \frac{d V_{2}}{d t}=\dot{x}_{2} A_{c 2}+Q_{w}
\end{aligned}
$$

This means that during the time domain simulation the displacement in the next time step $x^{n+1}$ is not only related to the velocity of of the column but also to the weir discharge.

$$
\begin{aligned}
& x_{1}^{n+1}=x_{1}^{n}+\left(\dot{x}_{1}^{n}-\frac{Q_{w}}{A_{c 1}}\right)\left(t^{n+1}-t^{n}\right) \\
& x_{2}^{n+1}=x_{2}^{n}+\left(\dot{x}_{2}^{n}+\frac{Q_{w}}{A_{c 2}}\right)\left(t^{n+1}-t^{n}\right)
\end{aligned}
$$


Now the velocity of the free-surface also does not equal to velocity of the column and the expressions for the convective acceleration and the mass change momentum are modified to:

$$
\begin{gathered}
\rho \frac{\dot{x}_{1}^{2}}{2} \rightarrow \rho \frac{\left(\dot{x}_{1}-\frac{Q_{w}}{A_{c 1}}\right)^{2}}{2} \\
\rho \frac{\dot{x}_{2}^{2}}{2} \rightarrow \rho \frac{\left(\dot{x}_{2}+\frac{Q_{w}}{A_{c 2}}\right)^{2}}{2} \\
\rho \frac{A_{c 1} x_{1}}{V_{c}} \dot{x}_{1}^{2} \rightarrow \rho \frac{A_{c 1} x_{1}}{V_{c}}\left(\dot{x}_{1}-\frac{Q_{w}}{A_{c 1}}\right)^{2} \\
\rho \frac{A_{c 1} x_{1}}{V_{c}} \dot{x}_{2}^{2} \rightarrow \rho \frac{A_{c 1} x_{1}}{V_{c}}\left(\dot{x}_{2}+\frac{Q_{w}}{A_{c 2}}\right)^{2}
\end{gathered}
$$

In further analysis of the weir discharge the drop of the water onto the free-surface results in an additional impact load on the receiving column, presented in figure 3.4.

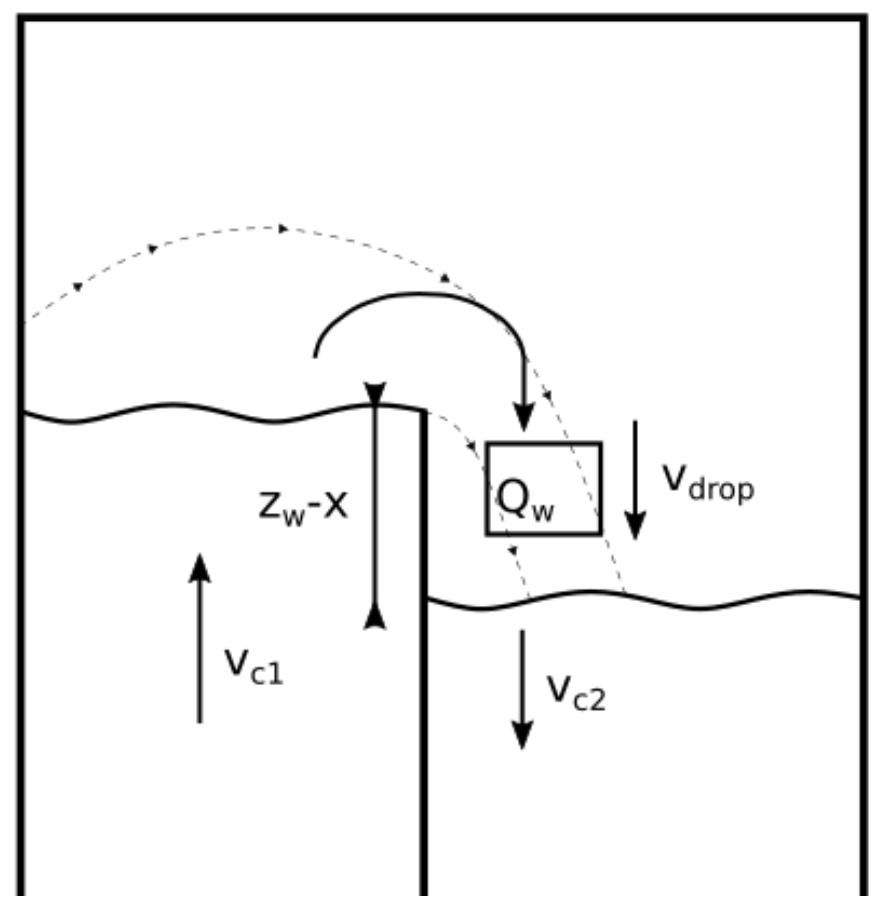

Figure 3.4: Scheme of impact load of the discharge weir

The impact is determined by the impact velocity and the discharge, where the velocity of the falling water is determined by the potential energy of at the weir level with respect to the free-surface of the impact:

$$
\rho g\left(z_{w}-x\right)=\frac{1}{2} \rho v_{d r o p}^{2}
$$

The impact velocity is the difference between the dropping water and the free surface is given by:

$$
v_{\text {impact }}=v_{\text {drop }}+\dot{x}
$$

The impact velocity multiplied by the mass flow over a weir result in a force. To obtain a pressure from this force, the impact is assumed to be spread over the full area of the free surface. In reality the impact is local would result in variation in the free surface, which is neglected using the rigid piston approach. The resulting pressures for on both columns are given by: 


$$
\begin{aligned}
& p_{\text {drop }, 2}=\begin{array}{cl}
\frac{\left(v_{\mathrm{drop}, 2}+\dot{x}_{2}\right) \rho\left|Q_{w}\right|}{A_{c 2}} & x_{1} \geq z_{w}, x_{2}<z_{w} \\
0 & x_{1}>z_{w}
\end{array} \\
& p_{\text {drop }, 1}=\begin{array}{cl}
\frac{\left(v_{\mathrm{drop}, 1}+\dot{x}_{1}\right) \rho\left|Q_{w}\right|}{A_{c 1}} & x_{2} \geq z_{w}, x_{1}<z_{w} \\
0 & x_{2}>z_{w}
\end{array}
\end{aligned}
$$

It is important to note that the impact is also assumed instantaneous because of the high complexity in modelling of the impact delay. Lastly, in equation (2.16) the relation between the free surface, weir level, the velocity and diameter was found for the flow out of a vertical circular pipe. In the modelling this effect has been neglected in assuming an instantaneous discharge. Additionally the velocity is decreasing during the weir discharge, for condition (a) in figure 3.3, resulting in a small effect of this additional pressure on the column.

\subsubsection{Air pressure}

Besides the mass transfer the columns are coupled by the pressure in the air chamber. The variation of pressure inside the chamber will give an additional pressure on the column; this pressure is given by $p_{a i r}$. For a static situation with the free surface level at its equilibrium position the air pressure is composed of the atmospheric pressure and the hydro static pressure. The mass of air contain in the air chamber equals $M_{\text {air }}=V(t) \rho(t)$, with $\rho_{\text {air }}(t)$ being the air density and $V(t)$ the air volume. The air chamber volume is given by the initial volume for the static situation and the inner free surfaces:

$$
V(t)=V_{0}-A_{c 1} x_{1}-A_{c 2} x_{2}
$$

For the trapped air the continuity equation is given by:

$$
\frac{d M_{a i r}}{d t}=\frac{d \rho(t) V(t)}{d t}=\dot{\rho}(t) V(t)+\dot{V}(t)=0
$$

To obtain the equation of motion assumptions regarding the thermodynamic within the air chamber have to be made. Given the small amplitudes of changes in pressure, compared to the total pressure, and the short time scale for heat exchange with the water an surrounding structure the thermodynamic process is assumed to be both adiabatic and reversible. This corresponds to a fully isentropic relation the air density and the air pressure, defined by:

$$
\left(\frac{p_{0}}{p(t)}\right)^{\frac{1}{\gamma}}=\left(\frac{\rho_{0}}{\rho(t)}\right)
$$

Where $p(t)=p_{o}+p_{\text {air }}, \rho_{0}$ and $\rho(t)$ are the initial and time dependent air densities and $\gamma$ is the polytropic exponent, for the isentropic process $\gamma=1.4$. From (3.37) the expression for the time dependent air density is given by:

$$
\rho(t)=\rho_{0}\left(\frac{p(t)}{p_{0}}\right)^{\frac{1}{\gamma}}
$$

Derivation of the air volume and density give

$$
\begin{gathered}
\frac{d \rho(t)}{d t}=\frac{d}{d t}\left(\rho_{0}\left(\frac{p(t)}{p_{0}}\right)^{\frac{1}{\gamma}}\right)=\frac{d p(t)}{d t} \frac{\rho_{0}}{p_{0} \gamma} \cdot\left(\frac{p(t)}{p_{0}}\right)^{\frac{1}{\gamma}-1} \\
\frac{d V(t)}{d t}=\frac{d}{d t}\left(V_{0}-A_{c 1} x_{1}-A_{c 2} x_{2}\right)=0-A_{c 1} \dot{x}_{1}-A_{c 2} \dot{x}_{c 2}
\end{gathered}
$$

Substituting (3.38), (3.39), (3.35) and (3.40) in the continuity equation (3.36) result in:

$$
\frac{d p(t)}{d t} \frac{\rho_{0}}{p_{0} \gamma} \cdot\left(\frac{p(t)}{p_{0}}\right)^{\frac{1}{\gamma}-1}\left(V_{0}-A_{c 1} x_{1}-A_{c 2} x_{2}\right)+\left(-A_{c 1} \dot{x}_{1}-A_{c 2} \dot{x}_{c 2}\right) \rho_{0}\left(\frac{p(t)}{p_{0}}\right)^{\frac{1}{\gamma}}=0
$$

Resulting in the final differential equation describing the air pressure in the air chamber:

$$
\dot{p}_{a i r}=\frac{\gamma\left(A_{c 1} \dot{x}_{1}+A_{c 2} \dot{x}_{c 2}\right)}{\left(V_{0}-A_{c 1} x_{1}-A_{c 2} x_{2}\right)}\left(p_{0}+p_{a i r}\right)
$$




\subsubsection{Hydrodynamic loads}

In this section the expressions for the hydrodynamic loads are derived. The hydrodynamic pressures are split into two parts, the first part is the wave excitation pressure and the second the radiation pressure.

\section{Wave pressure}

In this section the expressions for the wave pressure at the exit of the water columns is derived assuming linear wave theory. Starting with the continuity and momentum balance equations for a three dimensional control volume with directions $x, y$ and $z$.

$$
\begin{gathered}
\frac{\partial u_{x}}{\partial x}+\frac{\partial u_{y}}{\partial y}+\frac{\partial u_{z}}{\partial z}=0 \\
\frac{\partial\left(\rho u_{i}\right)}{\partial t}+\frac{\partial u_{x}\left(\rho u_{i}\right)}{\partial x}+\frac{\partial u_{y}\left(\rho u_{i}\right)}{\partial y}+\frac{\partial u_{z}\left(\rho u_{i}\right)}{\partial z}=F_{i}
\end{gathered}
$$

for $i=x, y, z$. Assuming a linear theory the momentum balance is simplified to

$$
\frac{\partial\left(\rho u_{i}\right)}{\partial t}=F_{i}
$$

The force on the RHS for each direction equals the pressure induced by both sides of the control volume:

$$
F_{i}=-\frac{\partial p}{\partial i}
$$

Substitution in the momentum balance equations results in the three linearity momentum equations.

$$
\begin{aligned}
\frac{\partial u_{x}}{\partial t} & =-\frac{1}{\rho} \frac{\partial p}{\partial x} \\
\frac{\partial u_{y}}{\partial t} & =-\frac{1}{\rho} \frac{\partial p}{\partial y} \\
\frac{\partial u_{z}}{\partial t} & =-\frac{1}{\rho} \frac{\partial p}{\partial z}-g
\end{aligned}
$$

The continuity and momentum equations are solved by the velocity potential with specific boundary conditions assuming irrotational flow. The velocity potential $\phi(x, y, z, t)$ is define such that is satisfies:

$$
u_{x}=\frac{\partial \phi}{\partial x}, u_{y}=\frac{\partial \phi}{\partial y} \text { and } u_{z}=\frac{\partial \phi}{\partial z}
$$

Substitution the directional velocities of the water particle in the continuity equations results in the equation called the Laplace equations.

$$
\frac{\partial^{2} \phi}{\partial x^{2}}+\frac{\partial^{2} \phi}{\partial y^{2}}+\frac{\partial^{2} \phi}{\partial z^{2}}=\nabla^{2} \phi=0
$$

The kinematic boundary conditions of at the free-surface and on the bottom can also be expressed by the velocity potential. Where the vertical velocity $u_{z}$ equals the speed of the surface elevation $\eta$ and equals zero at the impenetrable bottom:

$$
\begin{gathered}
u_{z}=\frac{\partial \phi}{\partial z}=\frac{\partial \rho u_{i}}{\partial t} \quad \text { at } z=0 \\
u_{z}=\frac{\partial \phi}{\partial z}=0 \quad \text { at } z=-d
\end{gathered}
$$

The momentum equations expressed with the velocity potential are given by:

$$
\frac{\partial}{\partial x_{i}}\left(\frac{\partial \phi}{\partial t}+\frac{p}{\rho}+g z\right)=0
$$

From this it can be seen that a arbitrary solution can be found for the term between brackets to equal zero: 


$$
\frac{\partial \phi}{\partial t}+\frac{p}{\rho}+g z=0
$$

This equation is the linearized Bernoulli equation for unsteady flow. From the Bernoulli equation the dynamic boundary condition at the free-surface can be derived for $Z=\eta$. Assuming a small surface elevation the boundary condition is assumed at $z=0$, regardless of the free surface elevation:

$$
\frac{\partial \phi}{\partial t}+g \rho u_{i}=0 \quad \text { at } z=0
$$

The velocity potential can be derived for a long-crested harmonic wave propagating in the horizontal y-directions the surface elevation can be expressed by:

$$
\eta(y, t)=a \sin (\omega t-k y)
$$

Additionally the velocity potential for waves in y direction only can be written as an expression with separated variables:

$$
\phi(y, z, t)=Y(y) Z(z) T(t)
$$

First the general solution is sought in:

$$
\phi(y, z, t)=Z(z) \cos (\omega t-k y)
$$

Substitution in the Laplace equation gives:

$$
\begin{gathered}
\nabla^{2} \phi=-k^{2} Z(z) \cos (\omega t-k y)+Z^{\prime \prime}(z) \cos (k y-\omega t)=0 \\
Z^{\prime \prime}(z)-k^{2} Z(z)=0
\end{gathered}
$$

This can be solved for the kinematic boundary condition at the bottom:

$$
\frac{\partial \phi}{\partial z}=0 \Rightarrow \frac{\partial Z(z)}{\partial z}=0 \text { at } z=-d
$$

Getting:

$$
Z(z)=A \frac{\cosh (k(z+d))}{\cosh (k d)}
$$

Where A is the unknown coefficient which can be solve for the dynamic boundary conditions at the free surface:

$$
\eta=-\frac{1}{g} \frac{\partial \phi}{\partial t}
$$

For the velocity potential given by:

$$
\phi(x, z, t)=A \frac{\cosh (k(z+d))}{\cosh (k d)} \cos (\omega t-k y)
$$

Next, substituting the dynamic boundary condition at $z=0$ into the kinematic conditions at $z=0$ gives:

$$
\frac{\partial^{2} \phi}{\partial t^{2}}+g \frac{\partial \phi}{\partial z}
$$
found:

Now, solving the Laplace equation together with the boundary conditions the particular solutions can be

$$
\phi=\frac{\omega a}{k} \frac{\cosh (k(d+z))}{\cosh (k d)} \sin (\omega t-k y)
$$

Together with the dispersion relation:

$$
\omega^{2}=g k \tanh (k h)
$$

Finally, substituting the solution for the velocity potential into the Bernoulli equation results in the equation for the total pressure: 


$$
p=-\rho g z+\rho g a \frac{\cosh [k(d+z)]}{\cosh (k d)} \sin (\omega t-k y) \quad \text { for } z<0
$$

The first term is the hydrostatic pressure, independent of the waves. The second term is the pressure induced by the waves.

This hydrostatic pressure will not excite the column, therefore only the dynamic wave pressure is used to determine the wave pressure on the columns. A uniform pressure over the full area of the mouth is assumed for wave lengths much longer than the width of the column and the incoming wave propagates parallel to the structure. Furthermore, an undisturbed wave field is assumed at both exits, because of the submerged structural time. This results to a pressure at the exit of the first column of:

$$
p_{w, 1}=\rho g a \frac{\cosh \left(k\left(-h_{1}+d\right)\right)}{\cosh (k d)} \sin \left(\omega_{w} t-k 0\right)
$$

Here the center first column is assumed at $y=0$ and $z=-h_{1}$. The resulting $y$-coordinate at the second column for $z=-h_{2}$ equals $y=L_{h}$, resulting in a phase difference between the wave pressure on the two columns. The pressure at the exit is now given by:

$$
p_{w, 2}=\rho g a \frac{\cosh \left(k\left(-h_{2}+d\right)\right)}{\cosh (k d)} \sin \left(\omega_{w} t-k L_{h}\right)
$$

\section{Radiation pressure}

In this section the hydrodynamic end-effects are determined. Due to the wave pressures at the exit and the air pressures in the chamber the water will oscillated, these oscillations accelerate the water around the exit and radiate waves. The radiation force can be decomposed which are in phase with the accelerations and the velocity of the motions of the water mass. In the frequency domain the radiation forces can be considered ass:

$$
F_{\text {rad }}=-\omega^{2} A(\omega) \tilde{X}+i \omega B(\omega) \tilde{X}
$$

Where $A(\omega), B(\omega)$ and $\tilde{X}$ are respectively the frequency dependent added mass, radiation damping and the amplitude. The derived equations of motion are highly non-linear for which the frequency domain approach is no longer applicable and is replaced by a time domain analysis. To obtain the expression for the radiation damping $B(\omega)$ in the time domain involves a convolution integral, the Cummins equation [35].

$$
F_{\text {rad }}=\int_{0}^{t} H(t-\tau) \dot{x} d \tau
$$

Here the integral represents the memory effect or influence of the mast motion radiation. The added mass is represented by the added mass at very high frequencies. $A(\omega) \approx A_{a d d, \infty}$. In this research the response in obtained for monochromatic waves only. Because the time domain response is only obtained for a single wave period the values for the added mass and the radiation damping are assumed for corresponding to that specific period:

$$
F_{r a d}(t)=B(\omega) \dot{x}
$$

and

$$
F_{\text {add }}=A \ddot{x}
$$

The complex geometry and the fact that the oscillating mass is also a fluid make it difficult to determine the radiation variables and require the application of numerical methods. Commercial codes based on the boundary element method (BEM) are available to compute the coefficients of ships and marine structures. These codes, like WAMIT, ANSYS/AQWA and Aquaplus, are also used frequently in the field of wave energy conversers. The coefficients in this report are determined via a different method. In [3] the hydrodynamic coefficients are determined using analysis by conformal mapping of local models. In this paper the coefficients have for the radiation damping $D_{r}$ and added length $l_{a}$ have been determined as a function of the wave length, the distance between the free-surface and the entrance to the ducts and the width of the duct perpendicular to the wave crest. The results have been validated in associate follow-up researches, in $[4,36]$. The grapps of the radiation damping and the added length are given in figures 3.5 and 3.6. In the figures $\lambda$ 


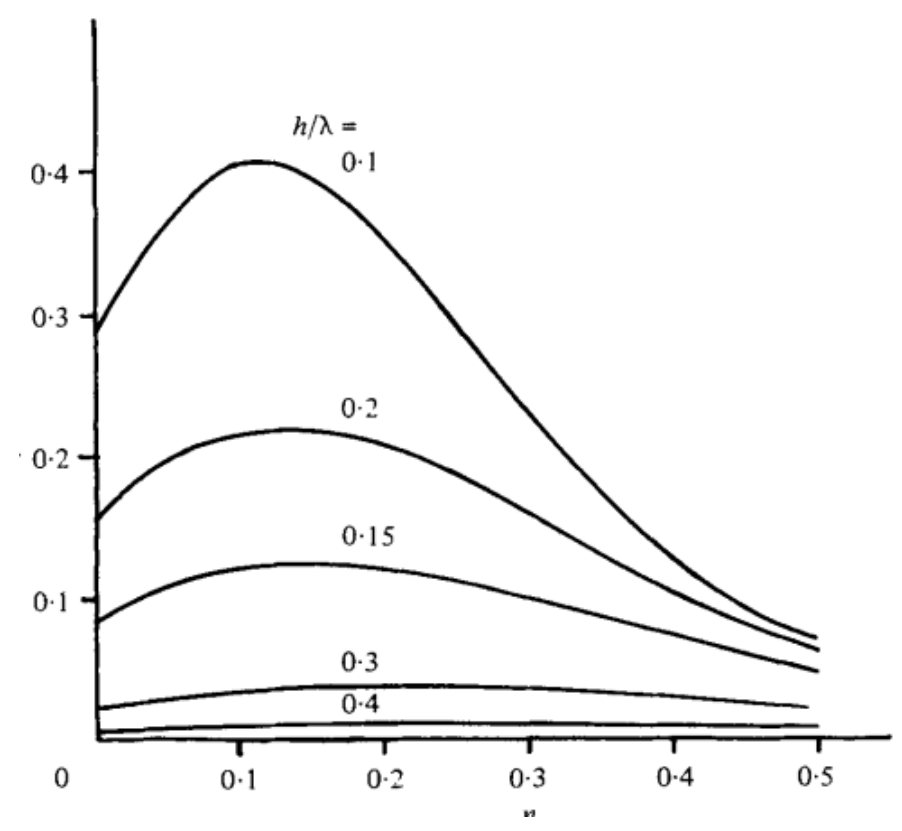

Figure 3.5: Radiation damping coefficient D, for mouth-upwards duct, calculated on two dimensional theory as a function of the widthwavelength ratio $n$ for different values of the depth-wavelength ratio $\frac{h}{\lambda}$. This coefficient $D_{r}$, is defined so that the energy radiated in new surface waves takes the form (3.73) per unit breadth, when the duct volume flow per unit breadth oscillates with amplitude $Q_{a}$ [3].

is the wave length, $l$ is the added length, $n=\frac{b_{c 1}}{L_{w}}$ is the ration between the width of the entrance to the wave length and $h$ the depth of the entrance. $D_{r}$ is determined for an energy disspation at rate:

$$
\frac{1}{2} \rho \omega D_{r}\left|Q_{a}\right|
$$

Where $Q_{a}=A_{c} \dot{x}$ is the unit flow rate.

To determine the corresponding coefficients to the calculation parameters the graphs are extrapolated in Matlab by the 'spline' method. From equation (3.73) and [3] the expression for the pressure induced by the radiation damping is derived for the time domain for a single excitation frequency:

$$
p_{r}=\rho \omega_{w} Q_{a} D_{r}=\rho \omega_{w}\left(A_{c} \dot{x}\right) D_{r}
$$

Here $D_{r}$ is given in $\left[m^{-} 1\right]$. The radiation damping coefficient $B\left(\omega_{w}\right)$ in equation (3.71) for pressures is now given by:

$$
B\left(\omega_{w}\right)=\rho D_{r}\left(h, n, L_{w}\right) \omega_{w} A_{c}
$$

The pressure related to the radiation damping of both columns is given by:

$$
\begin{aligned}
& p_{r, 1}=B_{1}\left(\omega_{w}\right) \dot{x}_{1}=\rho \omega_{w} D_{r}\left(h_{1}, \frac{b_{c 1}}{L_{w}}, L_{w}\right) A_{c 1} \dot{x}_{1} \\
& p_{r, 2}=\rho \omega_{w} D_{r}\left(h_{2}, \frac{D_{t}}{L_{w}}, L_{w}\right) A_{c 2} \dot{x}_{2}
\end{aligned}
$$

Now, the desired response, uni-directional flow, in the does not match response for which the coefficients are determined, oscillating flow. Therefore, the radiation damping of the second column is taken into account in the head loss associated to pipe flow at an exit to a large reservoir.

Additionally, the dimensions of the exit is far from the range of the variable in figure 3.6, the added mass can therefore not be determined accurately. In the terms for the acceleration of the second column it can be seen that the length of the small diameter exit governs the total term for the acceleration. This principle is also used to obtain the uni-directional flow, however the influence for large inertial term on the resonating first column is small. Therefore the added length is not determined for the second column, but an equivalent length is used. This equivalent length will be tuned for the desired response in chapter 4. 


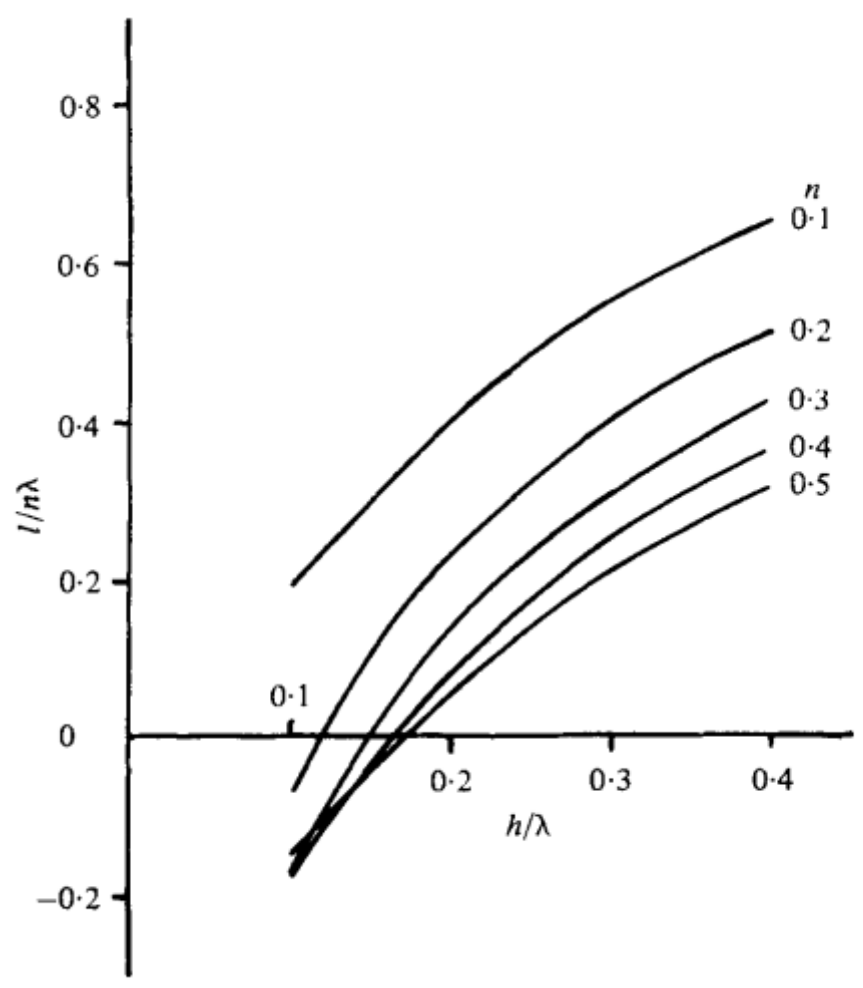

Figure 3.6: Added-mass curves in the mouth-upwards case, giving the ratio $\frac{l}{n \lambda}$ of the effective added length of duct to the width $n h$ of the duct mouth, as a function of the ratio $\frac{h}{\lambda}$ of mouth depth to wavelength. [3]

$$
L_{e q, 2}=\frac{A_{c 2}}{A_{2}}\left(L_{2}+L_{a, 2}\right)+L_{c 2}+x_{2}
$$

Finally, the pressure related to the added length of the first column is given by:

$$
p_{a, 1}=\frac{A_{c 1}}{A_{1}} l_{a, 1}\left(h_{1}, \frac{b_{c 1}}{L_{w}}, L_{w}\right) \ddot{x}_{1}
$$

The coefficients used in the calculation are presented in chapter 5.

\subsubsection{Hydraulic turbine}

In hydraulic power plants energy is generated in stationary conditions. However in the current model excited by periodic waves the system is dynamic. In conventional oscillating water column models air turbines are used to generate energy, these turbines can generate energy from a bi-directional flow allowing the model to work relatively efficient for oscillating flows. In the modelling the response of the dynamic system results can be estimate fairly accurate by simplified turbine models, or even linearized in the case of Wells turbines[7]. Water however, has a much larger density and viscosity than air and is much less incompressible. In stationary flows this results in a direct relation between the flow and the force giving high efficiencies in the power take-off. For dynamic oscillating system it gets more complicated. The design of a turbine for a dynamic system is outside of the scope of the project, therefore the modelled turbine for the dynamic response is derived from the regression relations of a stationary turbine highly simplified under the following assumptions.

- The turbine operates under a constant rotational speed

- Energy is extracted only for flow exiting the system, for the opposite flow direction the flow is considered to be undisturbed by the turbine.

Following these assumptions, the power generated by the turbine is calculated from the empirical regression relation between the specific speed $N_{s}$ and unit discharge $Q_{11}$ for bulb turbines found in [27]: 


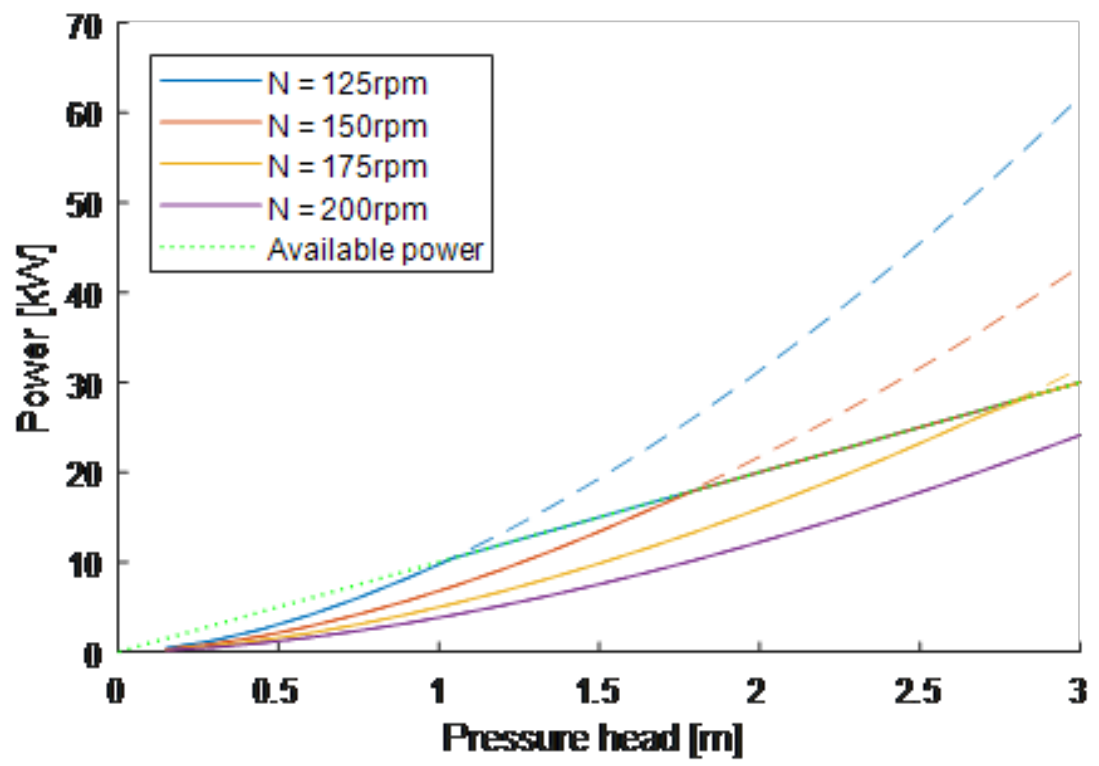

Figure 3.7: Relation between turbine power and water velocity for different rotational speeds according to empirical relations from Bulb turbines [27]; a turbine diameter of $2 \mathrm{~m}$ is used.

$$
N_{s}=k_{1} Q_{11}^{k_{2}}=\frac{N P^{0.5}}{H^{1.25}}
$$

Where $k_{1}=390.561$ and $k_{2}=0.8209$ are the coefficients for power take-off $P$ in $k W$ and rotation speed $N$ in $\mathrm{rpm}$. The unit discharge is given by:

$$
Q_{11}=\frac{Q}{D_{t}^{2} H^{0.5}} \approx \frac{A_{c 2} \dot{x}_{2}}{D_{t}^{2} H^{0.5}}
$$

For the stationary turbine the pressure head $H$, here in $m$, equals the stationary head of the water. In the dynamic conditions this head will accelerate and decelerate the water and will not be directly felt at the turbine. Therefore the dynamic pressure felt at the turbine will be used to calculate the response of the turbine:

$$
H=\frac{\frac{1}{2} \rho\left(\frac{A_{c 2}}{A_{2}} \dot{x}_{2}\right)^{2}}{\rho g}
$$

The final expression for the power take-off of the turbine is now given by:

$$
P_{t u r}=\left(k_{1}\left(\frac{Q}{D_{t}^{2} H^{0.5}}\right)^{k_{2}} \frac{H^{1.25}}{N}\right)^{2}
$$

Where both $Q$ and $H$ are time dependent. Finally, the pressure drop corresponding to the power take-off is calculated by:

$$
p_{t u r}=\frac{P_{t u r}}{\dot{x}_{2} A_{c 2}}
$$

In Fig. 3.7 the performance curve of the turbine is given for different velocities with a turbine diameter $D_{t}=2 \mathrm{~m}$. As a result of the simplified turbine model with a constant rotation speed, in the graph the output power is higher than the available power in the flow. An optimized turbine with a variable speed is capable to deal with this difference. In this report, the results with velocities higher than the limit velocity will be used to determine the potential of the device. To be able to indicate the results to be below or above the flow velocity limit, the limit velocities for different rotation speeds and turbine diameters are presented in appendix A.

Lastly, it is important to note that the turbine efficiencies are not taken into account. 


\subsubsection{Head losses}

The derivation of the equations of motion for the water columns the fluid was assumed inviscid and irrotational, in reality however, water is none of the two. To take the effects into account the head losses for friction and the minor head losses in the system. The friction losses at the walls are given by the Darcy-Weisbach equation.

$$
p_{\text {friction }}=f \rho \frac{L}{D_{\text {hydraulic }}} \frac{v^{2}}{2}
$$

Where $f$ is the Darcy friction factor, $L$ is the length of the section, $D_{\text {hydraulic }}$ is the hydraulic radius and $v$ the velocity in the section. In the first column the hydraulic radius is assumed big with respect to the length, therefore the friction factor is neglected.

The minor head loss equation is given by:

$$
p_{\text {loss }, 1}=\frac{1}{2} K_{w}\left(\frac{A_{c 1}}{A_{1}}\right)^{2} \dot{x}_{1}\left|\dot{x}_{1}\right|
$$

For the first column, the coefficient $K_{w} \approx 0.75$ was found for a similar kind of vertical column with a Ushape [37].

In the second column the diameter of the turbine is much smaller than the rest of the other column, this results in higher velocities and a smaller hydraulic radius, the friction factor cannot be neglected. The friction factor $f=0.024$ is assumed for a turbulent flow (Reynolds number ranging from $R e=2-6 \times 10^{6}$ ) using the modified version of the Colebrook White equation:

$$
f=\frac{0.25}{\left(\log \left(\frac{\epsilon}{3.7 \times D_{t}}+\frac{5.74}{R e^{0.9}}\right)\right)^{2}}
$$

Where a concrete roughness factor of $\epsilon=0.3$ and the preliminary turbine diameter of $D_{t}=2 \mathrm{~m}$ are used. Besides the friction the flow into the narrow tube introduces minor head losses, with the area of the first section of the column much large than the second section and a single direction flow, the losses are assumed for an inlet flow from a reservoir to a pipe section. To reduce the losses the transition to the pipe is assumed to be smooth, corresponding to a loss factor of $K_{w, \text { inlet }}=0.35$ [38]. As mentioned in the hydrodynamic section the damping at the exit will be taken into account by the exit loss. In general the head loss coefficient of an exit equals $\mathrm{K}=1$, where all kinetic energy is to be dissipated entering the surrounding water. At the turbine the velocities very height, causing a head loss equal to the kinetic energy resulting in a highly damped system. It is therefore important to reduce the head loss pressure in the exit. For this report a head loss coefficient equal to the inlet coefficient is assumed, with both coefficients related to the velocity through the turbine. The detailed design of the exit and the calculation of the hydrodynamic effects are outside the scope of this project. Therefore, the total head loss coefficient in the second column is assumed $K_{l o s s, 2}=0.75$ for both the entrance and exit effects as the internal friction.

\subsection{Calculation method}

The ordinary differential equations described above are solved numerically. The equations are non-linear and the exact solution can't be solved. To solve the equations approximated solutions can be such via numerical methods.

\subsubsection{Eulers method}

A common used method is the Euler's method. In this method the acceleration and velocity are determined for each time step, resulting in a time series response. To obtain the displacement $x$ and velocity $\dot{x}$ of each time step the Taylor series expansion is used:

$$
x(t)=x\left(t_{0}\right)+\left(t-t_{0}\right) \frac{\dot{x}\left(t_{0}\right)}{1 !}+\left(t-t_{0}\right)^{2} \frac{\ddot{x}\left(t_{0}\right)}{2 !}+\cdots
$$

For $t_{1}=t_{0}+\Delta t$ and $\Delta t \ll 1, t_{0}+\Delta t-t_{1}=\Delta t$.

$$
x\left(t_{1}\right)=x\left(t_{0}\right)+\Delta t \frac{\dot{x}\left(t_{0}\right)}{1 !}+\Delta t^{2} \frac{\ddot{x}\left(t_{0}\right)}{2 !}+O\left(\Delta t^{3}\right)
$$


For values of $\Delta t \ll 1$ the terms in the equation become progressively smaller proportional to the power of $\Delta t$. A similar approach can be done to obtain the velocity. However, the third derivative of the displacement $\dddot{x}$ cannot be calculated resulting in:

$$
\dot{x}\left(t_{1}\right)=\dot{x}\left(t_{0}\right)+(\Delta t) \frac{\ddot{x}\left(t_{0}\right)}{1 !}+O\left(\Delta t^{2}\right)
$$

The velocity error is larger than the displacement error. Since both outputs, the overall error is governed by the velocity. The displacement can also be generalized to a first order equation without introducing unnecessary large errors. Generalizing the equations for any time step $\mathrm{n}, t_{n}=t_{0}+n \Delta t$ :

$$
\begin{aligned}
& x^{n+1}=x^{n}+\Delta t \dot{x}^{n}+O\left(\Delta t^{2}\right) \\
& \dot{x}^{n+1}=\dot{x}^{n}+\Delta t \ddot{x}^{n}+O\left(\Delta t^{2}\right)
\end{aligned}
$$

Both equations can now be written in matrix form. When solved an approximation for the displacement and velocity is found by:

$$
\mathbf{u}^{n+1} \approx \mathbf{u}^{n}+\Delta t \dot{\mathbf{u}}^{n}
$$

Where:

$$
\mathbf{u}^{n}=\left[\begin{array}{c}
x^{n} \\
\dot{x}^{n}
\end{array}\right]
$$

In truncating the found equations a truncation error is introduces. The found equations are the solver for the ordinary differential equations according to the Euler's method. The error was $O\left(\Delta t^{2}\right)$ resulting in a second order solver.

\subsubsection{Runge-Kutta methods}

In this report the time series results are obtained using the Matlab function ODE 45, here the time RungeKutta method with a variable time step is implemented, a solver of the $4^{\text {th }}$ or $5^{\text {th }}$ order. The principle of the Runge-Kutta method is first explained for a $2^{\text {nd }}$ order problem, from which the higher order is easily explained. Consider the initial value problem [39]:

$$
\frac{d \mathbf{u}}{d t}=\mathbf{f}(t, \mathbf{u}), \quad \mathbf{u}=\mathbf{u}_{0} \quad \text { at } t=0
$$

The displacement is given by:

$$
\mathbf{u}^{n+1}=\mathbf{u}^{n}+a \mathbf{u}+b \mathbf{w}
$$

Where $\mathbf{u}^{n}=\mathbf{u}(n \Delta t)$,

$$
\mathbf{v}_{1}=\Delta t \mathrm{f}\left(t^{n}, \mathbf{u}^{n}\right) \quad \text { and } \quad \mathbf{v}_{2}=\Delta t \mathbf{f}\left(t^{n}+\alpha \Delta t, \mathbf{u}^{n}+\beta \mathbf{v}_{1}\right)
$$

$a, b, \alpha$, and $\beta$ are constants to be determined so that equation (3.94) is in agreement with the Taylor formula:

$$
\mathbf{u}^{n+1}=\mathbf{u}^{n}+\Delta t \frac{d \mathbf{u}}{d t} t^{n}+\frac{1}{2}(\Delta t)^{2} \frac{d^{2} \mathbf{u}}{d t^{2}} t^{n}+\frac{1}{6}(\Delta t)^{3} \frac{d^{3} \mathbf{u}}{d t^{3}} t^{n}+\cdots
$$

From the initial value problem in equations (3.93) the second derivative of the displacement can be written as:

$$
\frac{d^{2} \mathbf{u}}{d t^{2}}=\frac{\partial \mathbf{f}}{\partial t}+\frac{\partial \mathbf{f}}{\partial \mathbf{u}} \frac{d \mathbf{u}}{d t}
$$

Substituting the equations for the initial value in the Taylor formula (3.96) results the expression:

$$
\begin{aligned}
\mathbf{u}^{n+1}= & \mathbf{u}^{n}+\Delta t \mathbf{f}\left(t^{n}, \mathbf{u}^{n}\right)+\frac{1}{2}(\Delta t)^{2}\left(\frac{\partial \mathbf{f}}{\partial t}+\frac{\partial \mathbf{f}}{\partial \mathbf{u}} \mathbf{f}\right)\left(t^{n}, \mathbf{u}^{n}\right) \\
& +\frac{1}{6}(\Delta t)^{3}\left(\frac{\partial^{2} \mathbf{f}}{\partial t^{2}}+2 \frac{\partial^{2} \mathbf{f}}{\partial t \partial \mathbf{u}} \mathbf{f}+\frac{\partial^{2} \mathbf{f}}{\partial \mathbf{u}^{2}} \mathrm{ff}+\frac{\partial \mathbf{f}}{\partial \mathbf{u}} \frac{\partial \mathbf{f}}{\partial t}+\frac{\partial \mathbf{f}}{\partial \mathbf{u}} \frac{\partial \mathbf{f}}{\partial \mathbf{u}} \mathbf{f}\right)\left(t^{n}, \mathbf{u}^{n}\right) \\
& +O\left(\Delta t^{4}\right)
\end{aligned}
$$


A similar expression is found after substituting the expressions for $\mathbf{v}_{1}$ and $\mathbf{v}_{2}$ in the Taylor formula.

$$
\begin{aligned}
\mathbf{u}^{n+1}= & \mathbf{u}^{n}+(a+b) \Delta t \mathbf{f}\left(t^{n}, \mathbf{u}^{n}\right)+b \Delta t^{2}\left(\alpha \frac{\partial \mathbf{f}}{\partial t}+\beta \frac{\partial \mathbf{f}}{\partial \mathbf{u}} \mathbf{f}\right)\left(t^{n}, \mathbf{u}^{n}\right) \\
& +b \Delta t^{3}\left(\frac{1}{2} \alpha^{2} \frac{\partial^{2} \mathbf{f}}{\partial t^{2}}+\alpha \beta \frac{\partial^{2} \mathbf{f}}{\partial t \partial \mathbf{u}} \mathbf{f}+\frac{1}{2} \beta^{2} \frac{\partial^{2} \mathbf{f}}{\partial \mathbf{u}^{2}} \mathrm{ff}\right)\left(t^{n}, \mathbf{u}^{n}\right)+O\left(\Delta t^{4}\right)
\end{aligned}
$$

Comparing both equations implies:

$$
a+b=1, \quad b \alpha=b \beta=\frac{1}{2}
$$

Thus the coefficients can be taken as:

$$
a=b=\frac{1}{2} \quad \text { and } \quad \alpha=\beta=1
$$

The expression for the second order Runge-Kutta method becomes:

$$
\mathbf{u}^{n+1}=\mathbf{u}^{n}+\frac{1}{2}\left[\mathbf{f}\left(t^{n}, \mathbf{u}^{n}\right)+\mathbf{f}\left(t^{n+1}, \mathbf{u}^{n}+\Delta t \mathbf{f}\left(t^{n}, \mathbf{u}^{n}\right)\right)\right]
$$

Applying the same method result in the $4^{\text {th }}$ order Runge-Kutta method:

$$
\mathbf{u}^{n+1}=\mathbf{u}^{n}+\frac{1}{6}\left(\mathbf{v}_{1}+2 \mathbf{v}_{2}+2 \mathbf{v}_{3}+\mathbf{v}_{4}\right)
$$

For:

$$
\begin{aligned}
& \mathbf{v}_{1}=\Delta t \mathbf{f}\left(t^{n}, \mathbf{u}^{n}\right) \\
& \mathbf{v}_{2}=\Delta t \mathbf{f}\left(t^{n}+\frac{1}{2} \Delta t, \mathbf{u}^{n}+\frac{1}{2} \mathbf{v}_{1}\right) \\
& \mathbf{v}_{3}=\Delta t \mathbf{f}\left(t^{n}+\frac{1}{2} \Delta t, \mathbf{u}^{n}+\frac{1}{2} \mathbf{v}_{2}\right) \\
& \mathbf{v}_{4}=\Delta t \mathrm{f}\left(t^{n+1}, \mathbf{u}^{n}+\mathbf{v}_{3}\right)
\end{aligned}
$$

\subsubsection{Solving the matrix equation}

With the methods above the displacement and velocity can be determined for each time step. To be able to obtain both variables, the acceleration and velocity, of the time step should be solved. The acceleration can be determined by solving the matrix equation.

$$
\ddot{\mathbf{x}}\left(t_{n}\right)=\mathbf{M}^{-1}\left(\mathbf{F}\left(t_{n}\right)-\mathbf{C} \dot{\mathbf{x}}\left(t_{n}\right)-\mathbf{K} \mathbf{x}\left(t_{n}\right)\right)
$$

The velocity used to determine the displacement in time step $n+1$ is given by:

$$
\begin{aligned}
& \dot{x}_{1}^{\star}\left(t_{n}\right)=\dot{x}_{1}\left(t_{n}\right)-\frac{Q_{w}\left(t_{n}\right)}{A_{c 1}} \\
& \dot{x}_{2}^{\star}\left(t_{n}\right)=\dot{x}_{1}\left(t_{n}\right)+\frac{Q_{w}\left(t_{n}\right)}{A_{c 2}}
\end{aligned}
$$

Now the vector $\dot{\mathbf{u}}^{n}$ is known:

$$
\dot{\mathbf{u}}^{n}=\left[\begin{array}{c}
\dot{x}_{1}^{\star n} \\
\dot{x}_{2}^{\star n} \\
\ddot{x}_{1}^{n} \\
\ddot{x}_{2}^{n}
\end{array}\right]
$$

Subsequently the $\mathbf{u}^{n+1}$ can be solved by the numerical methods described above.

\subsubsection{Post-processing}

The ODE45 solves only gives the time dependent variables as an output, displacement, velocity and the air pressure difference. All other values such as the acceleration, weir discharge, power, pressures have to be calculated afterward in the post processing. 


\subsection{Conclusion}

In this chapter the theoretical derivation of the equations of motions were given. The final non-linear differential equations describing the motions of the water columns and the dynamics of the air chamber are:

$$
\begin{gathered}
\begin{array}{c}
\rho\left(\frac{A_{c 1}}{A_{1}}\left(L_{1}+L_{a, 1}\left(T_{w}\right)\right)+L_{c 1}+x_{1}\right) \ddot{x}_{1}+\left(\frac{A_{c 1} x_{1}}{V_{c 1}}+\frac{1}{2}\right) \rho\left(\dot{x}_{1}-\frac{Q_{w}}{A_{c 1}}\right)^{2}+\rho g x_{1}+p_{a i r} \\
=p_{w, 1}+p_{r, 1}+p_{\text {loss }, 1}-p_{\text {drop }, 2}
\end{array} \\
\begin{array}{c}
\rho\left(\frac{A_{c 2}}{A_{2}}\left(L_{2}+L_{a, 2}\left(T_{w}\right)\right)+L_{c 2}+x_{2}\right) \ddot{x}_{2}+\left(\frac{A_{c 2} x_{2}}{V_{c 2}}+\frac{1}{2}\right) \rho\left(\dot{x}_{2}+\frac{Q_{w}}{A_{c 2}}\right)^{2}+\rho g x_{2}+p_{\text {air }} \\
=p_{w, 2}+p_{\text {loss }, 2}+p_{\text {tur }, 2}-p_{\text {drop }, 2}
\end{array} \\
\dot{p}_{\text {air }}=\frac{\gamma\left(A_{c 1} \dot{x}_{1}+A_{c 2} \dot{x}_{c 2}\right)}{\left(V_{0}-A_{c 1} x_{1}-A_{c 2} x_{2}\right)}\left(p_{0}+p_{\text {air }}\right)
\end{gathered}
$$

\subsection{Discussion}

The model derived above is a representation of reality. Despite the non-linear equations of motions, several assumptions have been made in the derivation. A brief overview of the assumptions is given below.

- Linear wave theory is applied. In the derivation of the wave pressures, the fluid is assumed homogeneous, inviscid and incompressible, the surface tension is neglected, the flow is assumed irrotational, the waves are long-crested, and the amplitude is considered small. Furthermore, the pressures are calculated assuming undisturbed waves.

- The areas of the columns are assumed small with respect to the incident wavelengths. Uniform wave pressures and no vertical variations in the internal free surface are present. The effect of the weir discharge on the latter is neglected. Also, for larger free surface areas compared to the wavelength, this assumption losses its validity.

- The pressure inside the air chamber is assumed uniform. Also, ideal gas laws are applied, the compression and decompression of the internal air chamber are assumed an adiabatic reversible process. The non-linear air pressure is related to the flow in and out of the two columns.

- The radiation pressures of the first column are extrapolated from graphs in known literature. The shape effects of the specific structure are not taken into account. Reducing the accuracy of the values. The radiation damping in the second column is taken into account in the pressure losses coefficient, and the added length is assumed small compared to the total inertia of the column.

- To obtain the response of the turbine, a linear relation is assumed between the instantaneous dynamic flow state and the regression relation of stationary turbines for constant rotational speeds.

- For each column, a single pressure losses coefficient are approximated for the transitions in shape of the cross-section. The coefficient is applied to the highest velocity occurring in the column. After the detailed design, more valid parameters must be determined. 



\section{4}

\section{Model design}

In this section the dimensions for the proposed model are determined. First, a possible location is proposed and its environmental conditions found to determine the dimensions. Thereafter, the linear equations of motion are derived to obtain the linear natural frequencies and eigenmodes. Lastly, using the environmental conditions and the linear equations the inner dimensions of the model are determined.

\subsection{Environmental conditions}

In the final non-linear analysis the excitation force is modelled for monochromatic wave only. However, it is of interest of how the system would respond in a real wave climate. The system is therefore designed for environmental conditions found on a real location. In figure 2.1 of chapter 2 the west coast of the UK shows high average annual wave powers. Therefor the parametric design will be based on the environmental conditions associated with a location at the west coast.

\subsubsection{Site selection}

An important criterion for the site selection is the water dept. The wave pressures exciting the system decrease exponentially over the depth, in order to be able to generate the highest amount of energy the structure should not be submerged too deep. This moderate depth is found at several locations on the continental shelf, water depths ranging from $d_{w}=20-60 \mathrm{~m}$ are also found at the Hebrides Shelf, around the Hebrides Isles at the west of Scotland 4.1. A location in this area is assumed for the determination of the wave climate.

\subsubsection{Wave climate}

In the area of the desired locations several wave data results were found as shown in figure 4.2, NEXT grid point 15609 is used to analyse the wave climate. The wave rose and histogram of the wave direction, height and period at this location are presented in appendix B. The spectral peak period between $T_{p}=9$ and $10 \mathrm{~s}$ was observed and the mean annual significant wave height is $H_{s}=2.9 \mathrm{~m}$, the seasonal variation of significant wave height is between $H_{s}=1.7$ and $4.4 \mathrm{~m}$. The extreme significant wave height measured was $H_{s, \max }=$ $13.5 \mathrm{~m}$. The dominant wave directions are West and Southwest, with an occurrence of respectively $45 \%$ and $30 \%$.

\subsubsection{Depth}

As stated above the depth is an imported parameter to harvest energy from waves. If the structure is close to the free surface it will experience higher pressures than further below. To avoid exposure to the free surface at extreme conditions, exposing the structure to high impact loads, and to avoid collisions with vessels accidentally passing over the structure a minimum submerged depth is assumed, denoted by $h_{1}$ in figure 3.2. The maximum wave height equals approximately two times the significant wave height [11]:

$$
H_{\text {max }} \approx 2 H_{s}=27 \mathrm{~m}
$$

The maximum amplitude therefore equals $a_{\text {max }}=13.5 \mathrm{~m}$. From the maximum wave amplitude and an addition depth for safety, the submerged depth is set to $h_{1}=20 \mathrm{~m}$. Next to the minimal submerging depth, the 


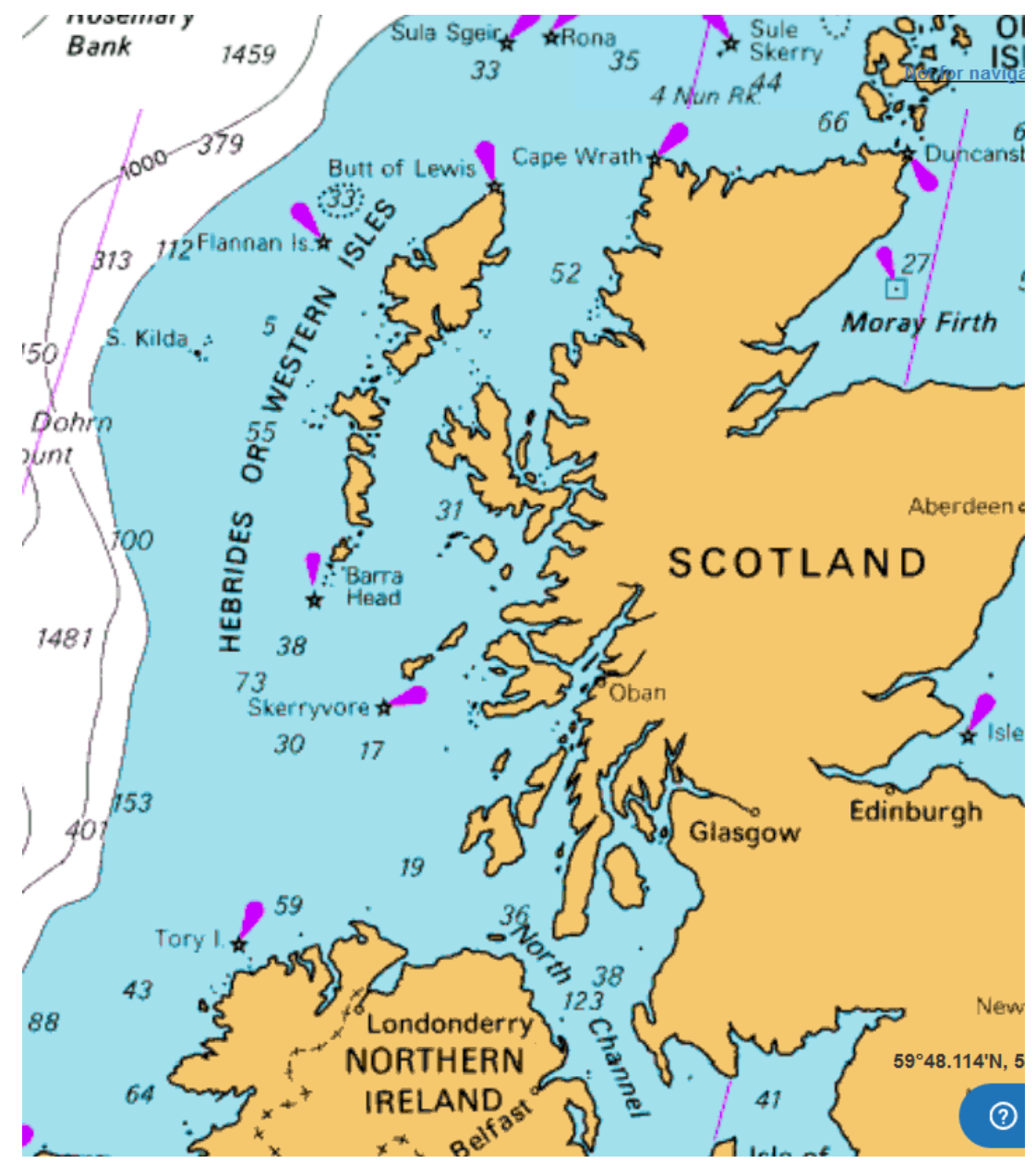

Figure 4.1: Water depths Hebrides Shelf [40]

inner dimension also require a large air chamber and the water level of the inner free surface may not be lower than the vertical side wall and discharge air from the air chamber. To take this space into account a structure height of again 20 meters will be used. The total depth used in the simulation there for is $d_{w}=40 \mathrm{~m}$. This depth is also found in the selected site.

\subsection{Linear equations of motion}

In this section the linear equations of motion are derived from equations (3.108), (3.109) and (3.110). The linear equations of motion will be used tune the inner dimensions of the model to resonate at the desired wave frequencies.

\subsubsection{Equations of motion of the water column}

First, the equations of motion of the two water columns are linearized. The first non-linear term that is disregarded is the weir discharge. The weir discharge decouples the velocity of the column to the displacement, the influence on the dynamics is highly non-linear. Besides the weir discharge the quadratic velocity terms related to the convective acceleration, change of mass and head losses are neglected. In the acceleration term the length of the water column is also dependent on the displacement of the column and is also ignored, together with the expression for the turbine in the second column. Lastly, the radiation damping in not taken into account. The resulting non-linear undamped equation of motion is given by: 


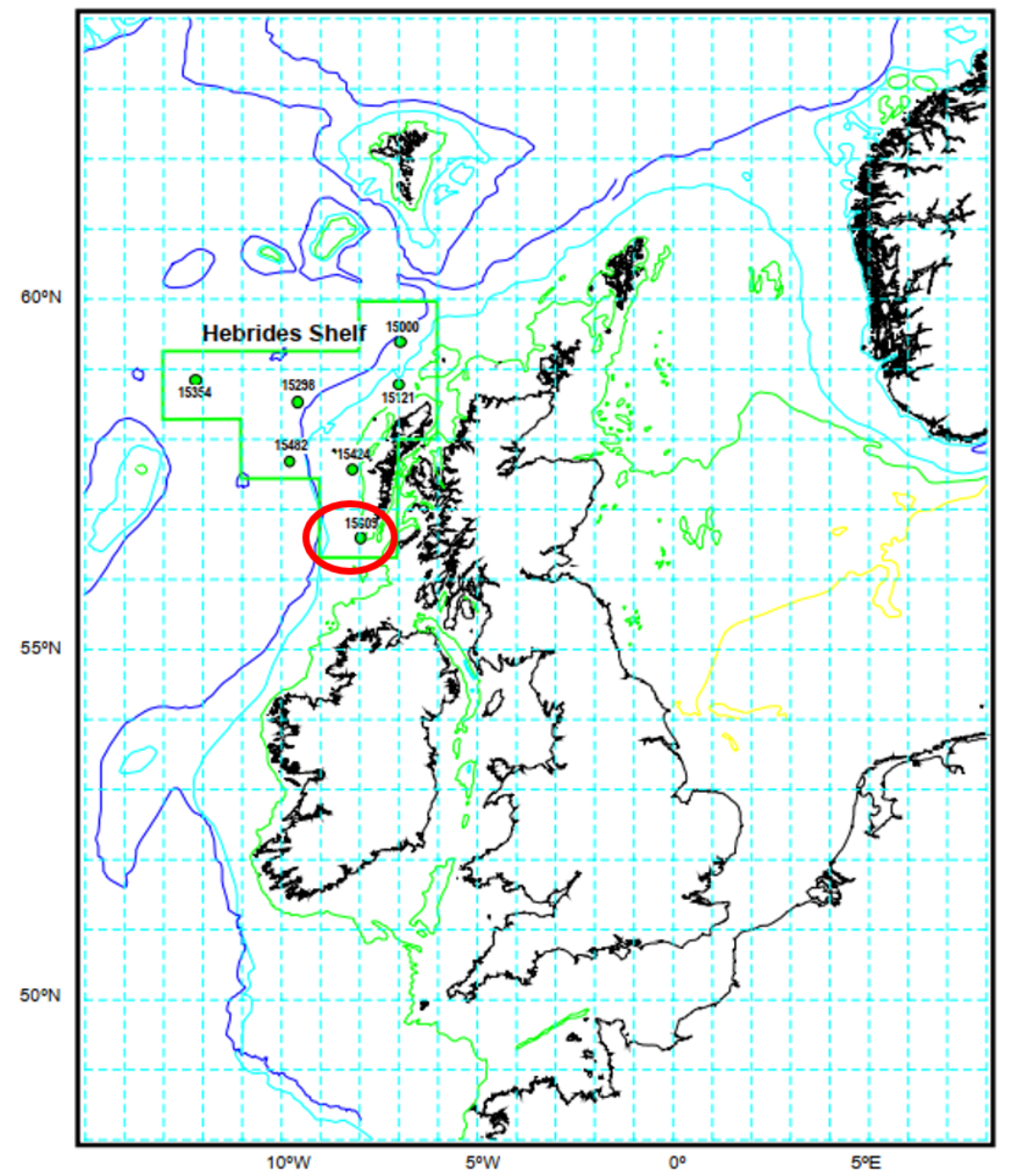

Figure 4.2: Location wave data Hebrides Shelf [41]

$$
\begin{aligned}
& \rho\left(\frac{A_{c 1}}{A_{1}} L_{1}+L_{c 1}+L_{a, 1}(\omega)\right) \ddot{x}_{1}+\rho g x_{1}+p_{a i r}=p_{w, 1} \\
& \rho\left(\frac{A_{c 2}}{A_{2}} L_{2}+L_{c 2}+L_{a, 2}(\omega)\right) \ddot{x}_{2}+\rho g x_{2}+p_{a i r}=p_{w, 2}
\end{aligned}
$$

\subsubsection{Equations of motion of the air pressure}

Next the equation describing the dynamic air pressre is linearized. First, small displacements of the two columns are assumed compared to the initial air volume:

$$
V_{0}>A_{c 1} x_{1}+A_{c 2} x_{2}
$$

Next, the initial pressure $p_{0}$ is assumed much large than the dynamic pressure difference:

$$
p_{0}>>p_{\text {air }}
$$

The equations of motion now holds:

$$
\dot{p}_{\text {air }}=\frac{\gamma p_{0}}{V_{0}}\left(A_{c 1} \dot{x}_{1}+A_{c 2} \dot{x}_{2}\right)
$$

With both terms dependent on the time derivative the linear expression for air pressure $p_{\text {air }}$ is found after the integration with respect to time.

$$
p_{a i r}=\frac{\gamma p_{0}}{V_{0}}\left(A_{c 1} x_{1}+A_{c 2} x_{2}\right)
$$


This equation can now be substituted in (4.2). Resulting in a problem with two linear equations of motion:

$$
\begin{aligned}
& \rho\left(\frac{A_{c 1}}{A_{1}} L_{1}+L_{c 1}+L_{a, 1}(\omega)\right) \ddot{x}_{1}+\left(\rho g+\frac{\gamma p_{0}}{V_{0}} A_{c 1}\right) x_{1}+\frac{\gamma p_{0}}{V_{0}} A_{c 2} x_{2}=p_{w, 1} \\
& \rho\left(\frac{A_{c 2}}{A_{2}} L_{2}+L_{c 2}+L_{a, 2}(\omega)\right) \ddot{x}_{2}+\left(\rho g+\frac{\gamma p_{0}}{V_{0}} A_{c 2}\right) x_{2}+\frac{\gamma p_{0}}{V_{0}} A_{c 1} x_{1}=p_{w, 2}
\end{aligned}
$$

\subsection{Linear analysis}

The three resulting linear equation are dependent on multiple different variables. This makes determining the dimensions difficult. In this section the sensitivity of each variable on the behaviour is analysed, with this information the final dimensions can be analysed.

\subsubsection{Starting points}

Previously in this chapter the depth of the water and the top of the structure are determined, concluding to a structural height of 20 meters. Next the rest of the dimensions, presented in figure 3.2 to tune the variables in the equations of motion are assumed. First the equilibrium level of the inner free-surface level is set to $h_{c}=30 \mathrm{~m}$. This results in an air chamber height of $h_{a i r}=10 \mathrm{~m}$. With these dimensions fixed the lengths of the vertical columns, $L_{1}, L_{c 1}$ and $L_{c 2}$ are also fixed, in [32] bottom boundary of the sections of the vertical columns were assumed at $\frac{1}{2} h_{\text {wall }}$, this dimension is however dependent on the areas of the column sections and will be determined in chapter 7 . Consequently, the bottom boundary of the vertical water columns are assumed a the bottom of the structure at depth $d_{w}$, resulting in model lengths of the columns by $L_{1}=20 \mathrm{~m}$, $L_{c 1}=10 \mathrm{~m}$ and $L_{c 2}=10 \mathrm{~m}$. For the modelling dimensions of the model the wall thickness of the structure is not taken into account, additionally assuming vertical walls and square corners the volume of the air chamber is described by:

$$
V_{0}=\left(A_{c 1}+A_{C 2}\right) h_{a i r}
$$

The thickness of the wall will be addressed in chapter 7 , here the outer dimensions will also be updated to accommodate the dimensions of the wall thickness together with the height of the opening between the vertical column sections. The center of the opening of the second column to the surrounding water is assumed 5 meters above the bottom level, $h_{2}=35 \mathrm{~m}$. In table the set global dimensions are summarized, including the used values for the atmospheric pressure, air density at atmospheric pressure at $T=20^{\circ} \mathrm{C}$, polytropic

\begin{tabular}{|c|c|c|}
\hline Variable & unit & value \\
\hline$d$ & $m$ & 40 \\
\hline$h_{1}$ & $m$ & 20 \\
\hline$h_{2}$ & $m$ & 35 \\
\hline$h_{c}$ & $m$ & 30 \\
\hline$L_{1}$ & $m$ & 20 \\
\hline$L_{c 1}$ & $m$ & 10 \\
\hline$L_{c 2}$ & $m$ & 10 \\
\hline$p_{a t m}$ & $\mathrm{~Pa}$ & $1 \times 10^{5}$ \\
\hline$\rho_{\text {air }}$ & $\mathrm{kg} \mathrm{m}^{-3}$ & 1.225 \\
\hline$\gamma$ & - & 1.4 \\
\hline$\rho$ & $\mathrm{kg} \mathrm{m}^{-3}$ & 1025 \\
\hline$g$ & $m s^{-2}$ & 9.811 \\
\hline
\end{tabular}
exponent, water density and gravitational acceleration.

Table 4.1: General Dimensions

\subsubsection{Frequency domain solution}

The spectral peak period in the area of the selected locations is between the 9 and 10 seconds, the spectral peak period is the period in which the energy of the total wave spectrum is a maximum. To optimize 
the power generation the system is to be designed for a resonance period of $T_{n}=9 \mathrm{~s}$. In earlier research a frequency reductions, increase of natural period, was found for a system including weir discharge, to account for this expected shift a linear natural period of $T_{n}=8.5 \mathrm{~s}$ is sought. To obtain the natural periods of the system the system is analysed in the frequency domain. First, the equations of motion are expressed in the matrix form:

$$
\mathbf{M} \ddot{\mathbf{x}}+\mathbf{K x}=\mathbf{p}
$$

Where $\mathbf{M}$ and $\mathbf{K}$ are given by:

$$
\mathbf{M}=\rho\left[\begin{array}{cc}
\frac{A_{c 1}}{A_{1}} L_{1}+L_{c 1} & 0 \\
0 & \frac{A_{c 2}}{A_{2}} L_{2}+L_{c 2}
\end{array}\right], \quad \mathbf{K}=\left[\begin{array}{cc}
\rho g+\frac{\gamma p_{0}}{V_{0}} A_{c 1} & \frac{\gamma p_{0}}{V_{0}} A_{c 2} \\
\frac{\gamma p_{0}}{V_{0}} A_{c 1} & \rho g+\frac{\gamma p_{0}}{V_{0}} A_{c 2}
\end{array}\right]
$$

And the displacement and force vectors by:

$$
\mathbf{x}=\left[\begin{array}{l}
x_{1} \\
x_{2}
\end{array}\right], \quad \mathbf{p}=\left[\begin{array}{l}
p_{w, 1} \\
p_{w, 2}
\end{array}\right]
$$

In this section the added mass of the system is neglected. The general solution is of the equations of motion is sought in the real form:

$$
x(t)=\sum_{i=1}^{2} \hat{x}_{1} A_{i} \sin \left(\omega_{i} t+\phi_{i}\right)
$$

The corresponding eigenfrequency problem is:

$$
\left(-\omega_{i}^{2} \mathbf{M}+\mathbf{K}\right) \hat{x}_{i}=0
$$

By solving this equation the natural frequencies $\omega_{i}$ and eigenvectors $\hat{x}_{i}$ are obtained. For the initial analysis, to obtain the values of the natural frequencies and the eigenvectors, the mass and stiffness matrices are made with the random dimensions in the equations of motion. These dimensions are based on the properties required to obtain the wanted dynamic behaviour of the system. This behaviour consists of, as describe in chapter 1, a resonating first column and a less dynamic second column with only outgoing flow as a result of the weir discharge from column 1 to column 2. The reduction in dynamic response can be obtained by using a small area in the exit pipe of the second column with respect to its inner free-surface. The expression $\frac{A_{c 2}}{A_{2}} L_{2}$ is now dominant with respect $L_{c 2}$ and the value of $\mathbf{M}(2,2)$ becomes much larger than the value of $\mathbf{M}(1,1)$. In order to minimize the water discharge from the second column to the first the area of the second is chosen bigger than the area of the first column. Now more water volume is required for the inner free-surface level of the second column $x_{2}$ to reach the weir level. This also increases the factor $\frac{A_{c 2}}{A_{2}}$ in the mass matrix resulting in a higher inertia of the second column. The used dimensions for the initial analysis of the response of the system are given in Table 4.2, note that the dimensions are determined per meter width of the structure.

Table 4.2: Dimensions initial frequency analysis per meter width

\begin{tabular}{|c|c|c|}
\hline Variable & unit & value \\
\hline$L_{c 1}$ & $m$ & 10 \\
\hline$L_{1}$ & $m$ & 20 \\
\hline$L_{c 2}$ & $m$ & 10 \\
\hline$L_{2}$ & $m$ & 10 \\
\hline$A_{1}$ & $m^{2}$ & 4 \\
\hline$A_{c 1}$ & $m^{2}$ & 4 \\
\hline$A_{c 2}$ & $m^{2}$ & 8 \\
\hline$A_{t}$ & $m$ & 0.314 \\
\hline$V_{0}$ & $m^{3}$ & 120 \\
\hline
\end{tabular}

The resulting eigenperiods and eigenvectors are: 


$$
\begin{array}{cc}
T_{1}=6.37 s & \hat{x}_{1}=\left[\begin{array}{c}
1 \\
0.0869
\end{array}\right] \\
T_{2}=22.69 s & \hat{x}_{2}=\left[\begin{array}{c}
-1 \\
0.7002
\end{array}\right]
\end{array}
$$

In the two modal shaped some clear differences are observed. The first mode has a much lower eigenperiod and the values of the eigenvectors are both positive. This in-phase motion of the columns results in a high change of volume of the air chamber, increasing the amplitude of the pressure oscillations. In the second mode a much higher eigenperiod is found in combination opposing directions of of the DOFs in the eigenvector. The out of phase motion means smaller changes in volume. Looking at the natural period of a simple one degree of freedom (DOF) system:

$$
T_{n}=2 \pi \sqrt{\frac{m}{k}}
$$

Where $\mathrm{m}$ is the mass of the system and $\mathrm{k}$ is the restoring force. For higher amplitudes of the pressure oscillations the air pressure represents a stiffer restoring force, explaining the big difference in eigenperiod of the two modes. The other difference between the two modes is the magnitude of the second DOF in the eigenvector. In the first mode the value corresponding to the second column is much smaller than the value of the first column, where the second mode the difference is in the same order of magnitude as the ration of the inner free-surface areas.

Concluded, in the first mode the first column is more dynamic than the second column, this effect is earlier in this report noted as a requirement for the system to work. Therefore, the system will be tuned so that mainly the first mode is excited by the desired wave periods. The natural period of this mode is to be increased. For the small dynamic response the second column is in this section assumed to be infinite stiff. Consequently, the system can be described as a simple one DOF system where the restoring force and the mass can be described by:

$$
\begin{aligned}
k & =\rho g+\frac{\gamma p_{o}}{V_{0}} A_{c 1} \\
m & =\rho\left(\frac{A_{c 1}}{A_{1}} L_{1}+L_{c 1}\right)
\end{aligned}
$$

To increase the natural period the mass should be increased or the stiffness should be decreased. The stiffness of the gravitational force is independent on the dimensions and can there for not be changed A reduction of the stiffness can therefor only by obtained by reducing the initial pressure and free surface area of increasing the initial volume. The mass term can be increased by increasing the length of the column or by decreasing the ration $\frac{A_{c 1}}{A_{1}}$. The outer dimensions have been determine earlier, the lengths of the column are fixed. By changing the level of the free surface $V_{0}, L_{C 1}$ and $L_{c 2}$ change. The level of $h_{c}=30 \mathrm{~m}$ was chosen to avoid the free surface getting below the vertical wall. From the analysis was found that the natural period increased for a lower free-surface level, increasing the chance of the oscillating column to reach below the vertical wall. Therefore $h_{c}$ is kept at $30 \mathrm{~m}$. From this starting point, the initial pressure and the lengths of the vertical columns cannot be changes. Consequently, the natural period can only be increased by tuning $A_{1}$, $A_{c 1}, A_{c 2}, A_{2}$ and $L_{2}$. Decreasing $A_{1}$ will increase the ration $\frac{A_{c 1}}{A_{1}}$ increasing the mass term. Increasing $A_{c 1}$ and $A_{c 2}$ will increase the air volume $V_{0}$, reducing the stiffness. With $A_{c 1}$ in the nominator in the terms of both the mass and the stiffness it will also increase the stiffness and the mass.

In figure 4.3 the natural period for different dimensions are presented for the t. The natural period is increased exponentially for a reduction of $A_{1}$ and linearly for an increase of $A_{c 1}$ and $A_{c 2}$. The increase of stiffness of the second column, by reducing $A_{2}$ or increasing the $L_{2}$, was found to have little effect on the natural frequency in this mode. However, the inertia term is found to be important in the dynamics of the full system, a high inertia gives lower oscillations of the flow in the second column, combined with a high weir discharge resulting in a uni-directional flow. From this analysis it is seen that the increase of the initial air volume $V_{0}$ and the ratio $\frac{A_{c 1}}{A_{1}}$ result in an increase of the natural frequency. In determining the final dimensions the increase of air volume is sought in the increase in the air of the free-surface of the second column, to remain with $A_{c 2}>A_{c_{1}}$ and increase the ratio $\frac{A_{c 2}}{A_{2}}$, increasing the inertial of the second column. Besides decrease of stiffness of the air chamber the inertial term of the first column is increased by the ratio $\frac{A_{c 1}}{A_{1}}$. 


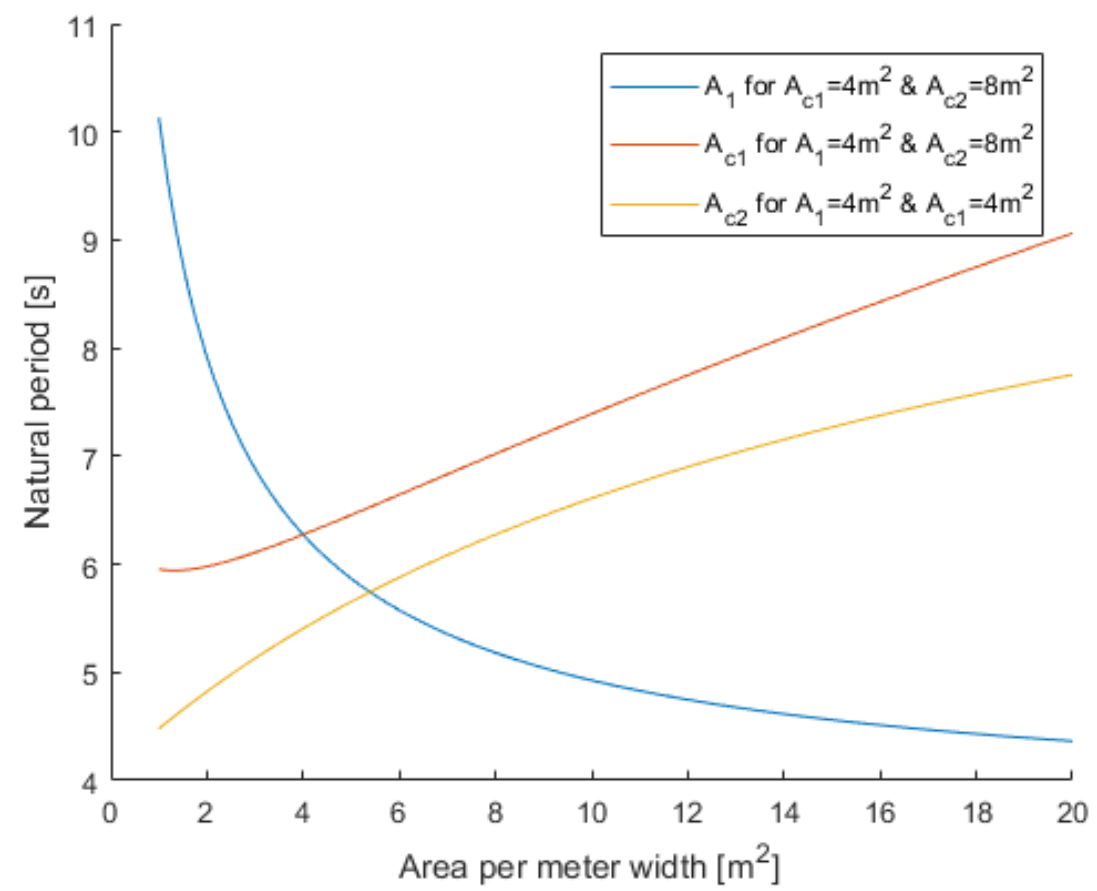

Figure 4.3: Dependence of natural period on the column areas met meter with

\subsubsection{Dimensions}

In this section the proposed dimensions of the model with a natural frequency of 8.5 seconds are presented. In this section the added length corresponding to a $T_{w}=8.5$ is taken into account. From the relations found in the previous section two different geometries are found through multiple iteration phases. The optimal relation of for the inner free-surface area's $A_{c 2}=4 A_{c 1}$ was found. This relation resulted in an air volume big enough to reduce the stiffness and remain with an area of $A_{1} \propto A_{c 1}$. Observed in the section above the inertia of the second column is governed by $\frac{A_{c 2}}{A_{2}} L_{2}$ confirming the statement in chapter 3 . In table 4.2 geometries are given including the equivalent length determined in equation (3.77). It is important to note that this research is executed for a width of $10 \mathrm{~m}$.

Table 4.3: Dimensions initial frequency analysis for a structure width of 10 meter, corresponding to 3.2

\begin{tabular}{|c|c|c|}
\hline Variable & unit & Geometry 1 \\
\hline$L_{c 1}$ & $m$ & 10 \\
\hline$L_{1}$ & $m$ & 20 \\
\hline$L_{a, 1}\left(T_{w}=9 s\right)$ & $m$ & 1.67 \\
\hline$L_{e q, 2}$ & $m$ & 800 \\
\hline$A_{1}$ & $m^{2}$ & 28.5 \\
\hline$A_{c 1}$ & $m^{2}$ & 40 \\
\hline$A_{c 2}$ & $m^{2}$ & 160 \\
\hline$V_{0}$ & $m^{3}$ & 2000 \\
\hline
\end{tabular}

The resulting natural periods and eigenvectors of the proposed geometry are:

$$
\begin{array}{cc}
T_{1}=8.5 s & \hat{x}_{1}=\left[\begin{array}{c}
1 \\
0.0287
\end{array}\right] \\
T_{2}=33.11 s & \hat{x}_{2}=\left[\begin{array}{c}
-1 \\
0.4399
\end{array}\right]
\end{array}
$$

From the analysis it was found that if the free-surface areas are scaled by $A_{c 2}=4 A_{c 1}$ the stiffness of the 
air pressure is the same. This can be important in the scaling of the structure, in the next chapter the power output of the non-linear response for smaller cross-section will be compared to the power of the proposed dimensions. The next chapter will also analyse the response excluding a turbine, focusing on the effect of the change in mass in the system.

\subsection{Conclusion}

In this section the starting points for the non-linear analysis have been determined. The wave conditions have been analysed for a possible location with a high annual wave energy density. The spectral energy peak periods of 9 to 10 seconds were found with a monthly mean significant wave height varying between the 1.7 and $4.4 \mathrm{~m}$. The proposed model will be analysed for wave characteristics around this range of values. The system is tuned for a resonance period of 8.5 seconds for the linearized equations of motion. The non-linear resonance of the system is assumed to resonate for higher periods caused by the weir discharge, in this period region a higher energy density is expected according to the peak period. The model is tuned for a water depth of 40 meter and a structural height of 20 meter. The final dimensions used in the non-linear time domain model are presented in table 4.3. 


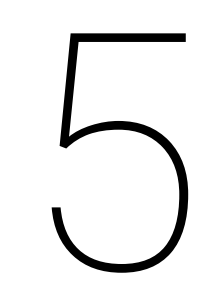

\section{Time domain results}

In this chapter, the results of the time series obtained by time domain modelling are presented. Various cases are addressed, starting with the response to regular waves excluding weir flow and power take-off for comparison with the results for the linear equations of motions derived in the previous chapter. Subsequently, the effect of weir discharge is analysed, comparing the response of different weir levels to the response without weir flow. After the analysis of the general behaviour, the influence of the power take-off is presented, and the performance for different incoming wave amplitudes is found.

\subsection{Wave parameters, forces and hydrodynamic coefficients}

In this section, input variables for the simulations are presented, following the equations and graphs presented in chapters 2 and 3. In this research, the response is obtained for regular waves only; the first part of the analysis will only present results for regular incoming waves with an amplitude of $a_{w}=2 \mathrm{~m}$. In a later section, the response for different wave amplitudes will be given.

The results obtained in the following sections will be presented for wave periods in between the 6 and 13 seconds. The wavelengths and wave numbers for the given depth are shown in figure 5.1.

The different periods also results in different dynamic wave pressures at the openings of the column to the surrounding water. In figure 5.2 the undisturbed wave pressure felt by the columns at the openings at depths $h_{1}$ and $h_{2}$ are presented.

Lastly, from the wave parameters and the dimensions, the added length and radiation damping are obtained, shown in figure 5.3.

\subsection{Response excluding power take-off}

In this section, the time series results are presented excluding the power take-off. In the first part of this section, the response without weir discharge is given and compared to the frequency response function of the linear equations of motion derived in the previous chapter. After identifying this response, the effect of the weir discharge and mass transfer between the columns is analysed. In this section, the results are obtained for a diameter of the exit pipe of $D_{t}=2 \mathrm{~m}$.

\subsubsection{Response excluding weir discharge.}

In models of the oscillating water column the equations of motion are often linearised. The numerical results were found to correspond reasonably with the experimental results, to main inaccuracy was found in the linearization of the non-linearity of the turbine response and the inhalation of air to the chamber. Therefore it is assumed that the time series results found for a system without weir discharge will correspond closely to the linear equations of motion for the same problem. In this part, the results are shown with and without the pressures losses caused by the shape, friction and exit/entrance losses. The results without pressure losses are called the undamped system, and the results including pressure losses are addressed by the damped system. The hydrodynamic effects in the first column are taken into account. 

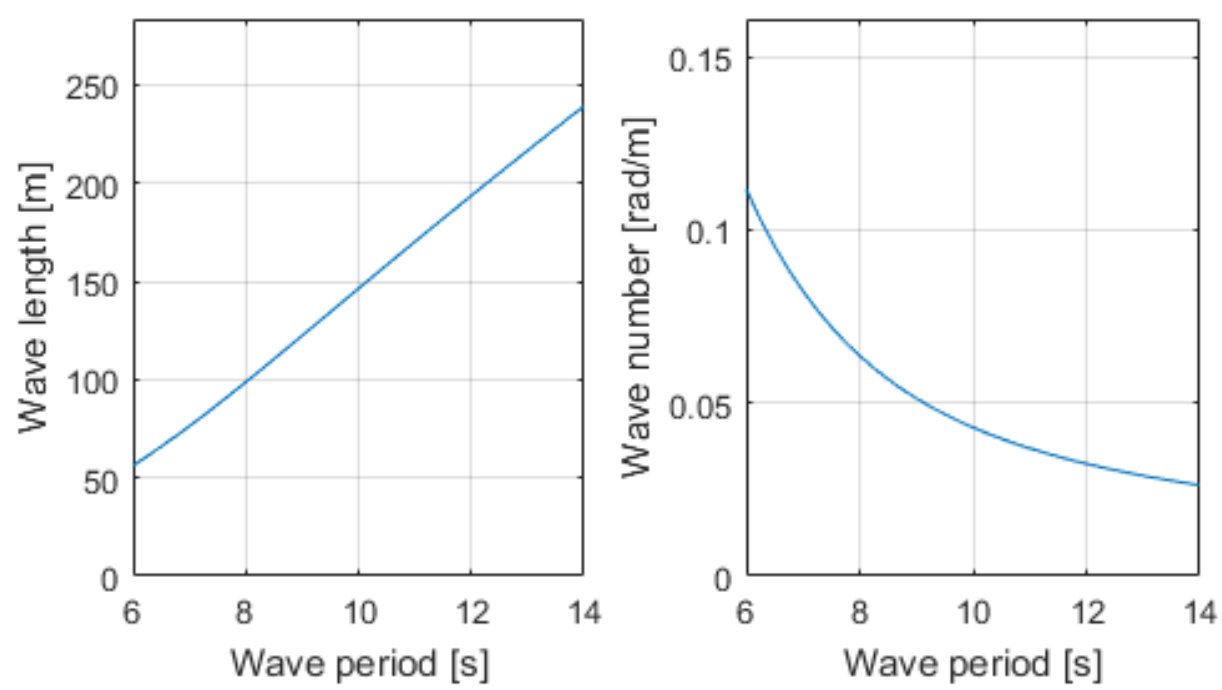

Figure 5.1: Wave length (left) and wave number (right) for different wave periods for a depth of $d=40 \mathrm{~m}$

\section{Time series}

In figure 5.4 and 5.5 the respectively undamped and damped time series are shown at $t \gg 0$, representing the steady state response. The time axis is normalized by the natural period of the system $T_{n}=8.5 \mathrm{~s}$. In the figures the response of three different excitation periods are presented, $T_{w}=8,8.5$ and $9 \mathrm{~s}$. Where the period of $8 \mathrm{~s}$ is below the natural period, $8.5 s$ is the natural period and $9 s$ is above the natural period.

The phase of the different excitation periods is as expected. In the two figures, the phase of the resonance periods is between the two other responses with the lower period lagging.

The phase of the excitation forces is presented in the discharge graphs. Comparing the damped and undamped response of the resonance frequency, the effect of the damping is visible. The displacements of the undamped system are much higher than the damped system, despite the phase of the discharge lagging behind the forcing while the phases are in line for in the damped response. If looking at the motion at the start of the simulations of the undamped model, shown in 5.6, the phase of the motion is in line with the forcing, however, for increasing discharges and displacements the phase is drifting off.

This can be explained by the non-linear parts of the equations. The large displacements also influence the inertia of the resonating column, with a positive displacement increasing the mass and natural period, while a negative displacement decreases the natural period. Eventually, the difference between will be cancelled out, expecting a total response still in phase with the forcing. There is however, also the changing restoring force of the air pressure, the air pressure is a so-called stiffening spring for volume reduction and a softening spring for volume increase. The positive displacements and the corresponding stiffness will, therefore, have a more significant effect than the less stiff spring constant for negative displacements, results in a net increase of spring stiffness. This increase decreases the natural period, resulting in the lagging phase response at the resonance period and displacement not going to infinity.

The stiffening of softening effect is more prominent for large displacements. Therefore the phase of the damped model is in more accordance with the forcing because the total displacements are smaller.

Lastly, the small response of the second column, indicated by the dashed lines, is in agreement with the statements made in the design of the structure; the high inertia in the second column results to a small response.

\section{Linear vs non-linear model}

In following the maximal displacement values of the time series results are compared to the frequency response of the linear equations of motions to a wave forcing at the first column only, $F_{w, 2}=0$. The results in figure 5.7 and 5.8 are in agreement with the expectation; the linear model can approximate the non-linear model closely. As expected, the linear undamped frequency response has a much large value at the resonance period, but are almost the same as the undamped model at all other periods. In the damped model, the frequency response is found to overestimate the response slightly for periods below the natural periods and underestimate the response for higher periods. 

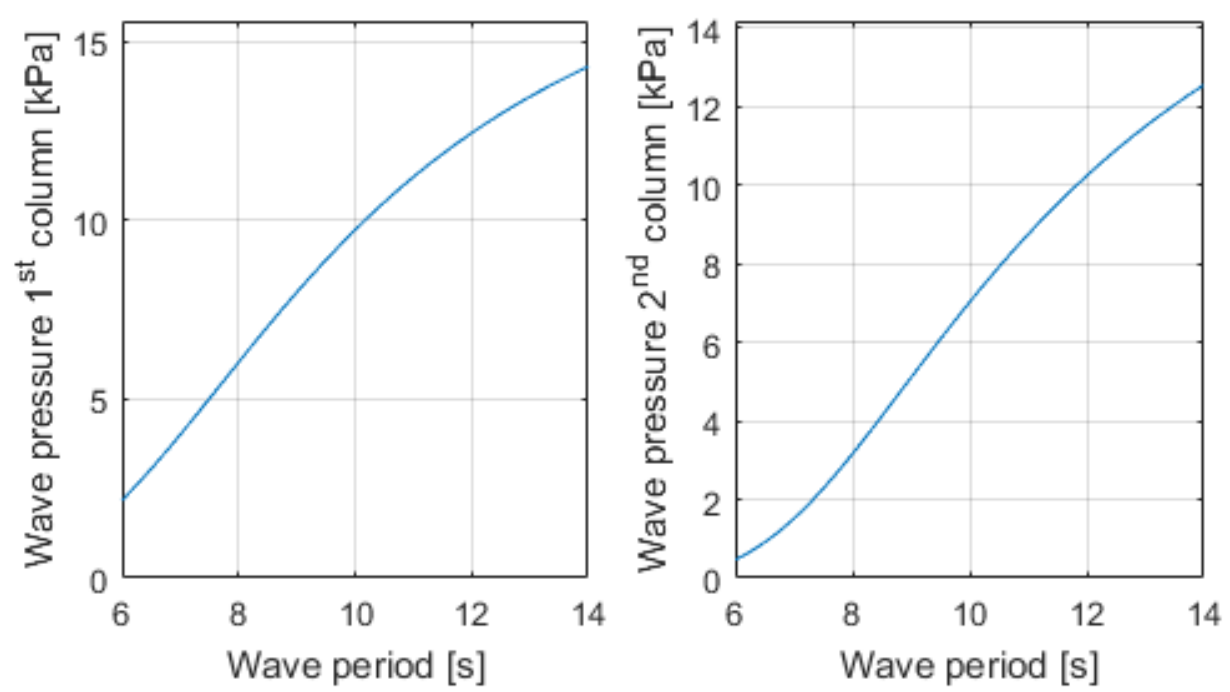

Figure 5.2: Wave pressures on column 1(left) and column 2 (right)
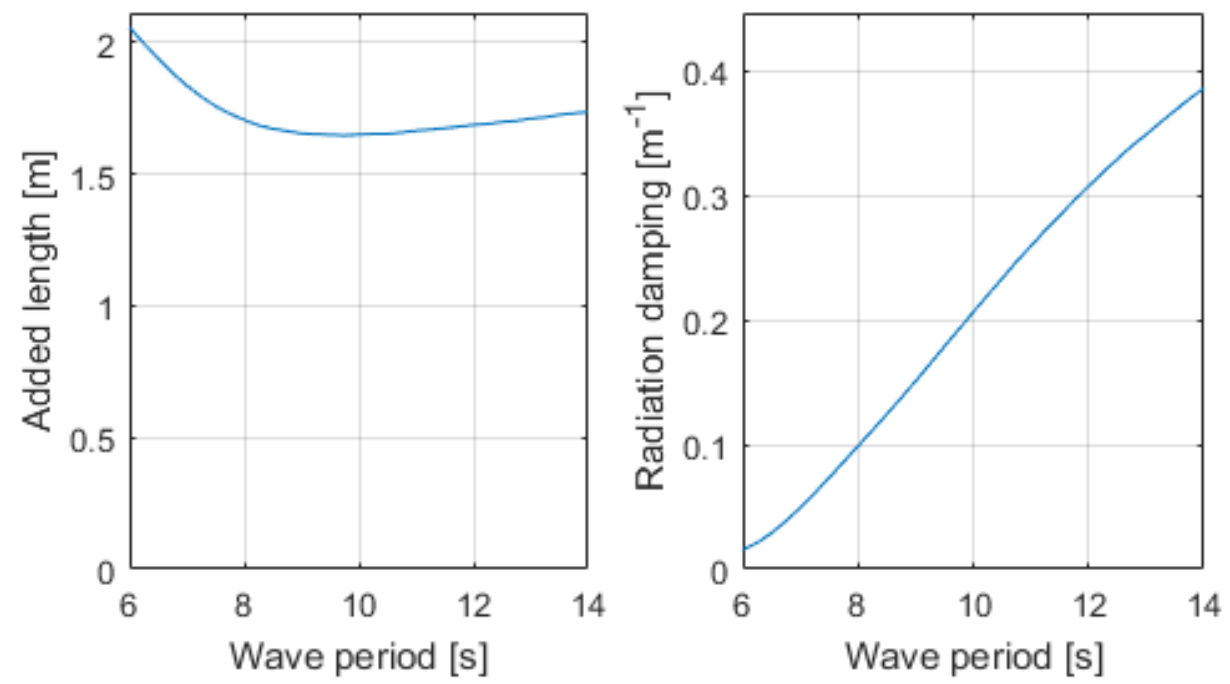

Figure 5.3: Hydrodynamic coefficients: Added length $L_{a}$ (left) and Radiation damping $D_{r}$ (right) 


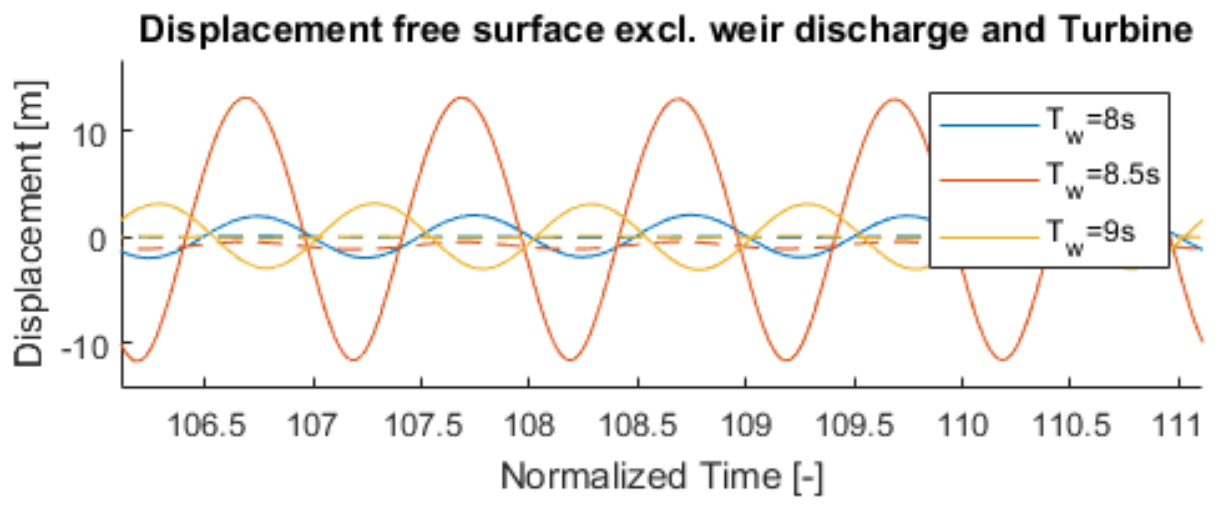

Discharge in columns excl. weir discharge and Turbine

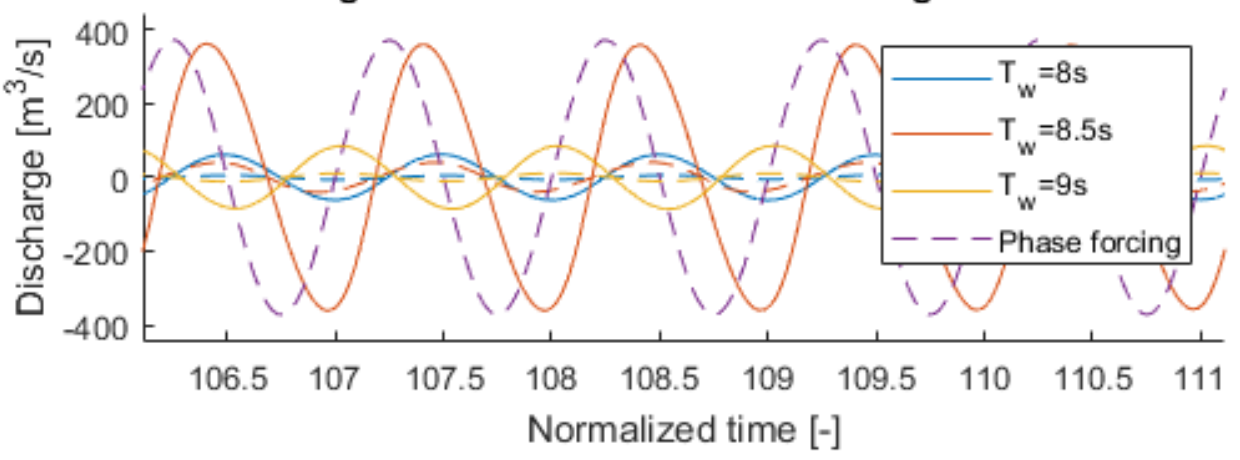

Figure 5.4: Time domain results undamped system without weir flow

Displacement free surface excl. weir discharge and Turbine

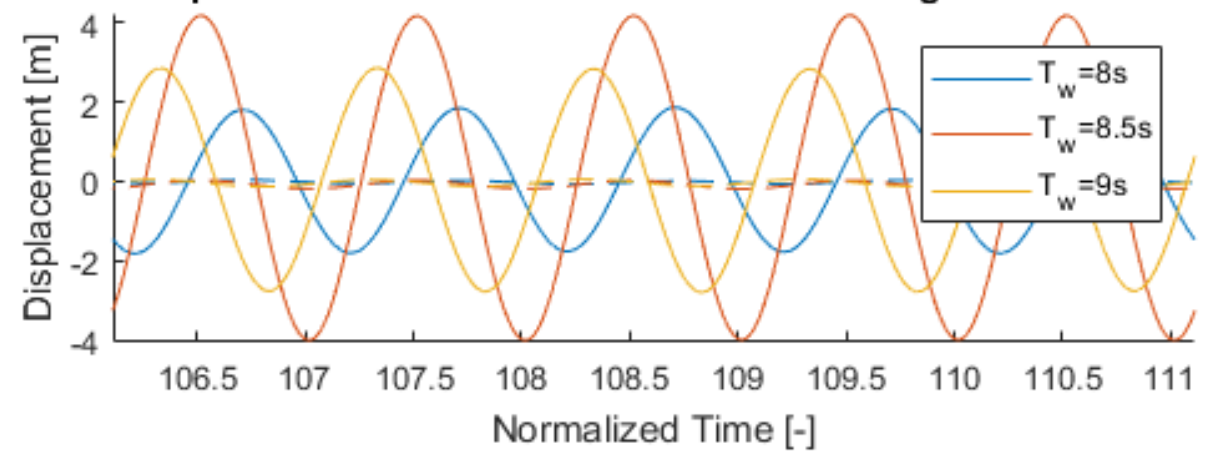

Discharge in columns excl. weir discharge and Turbine

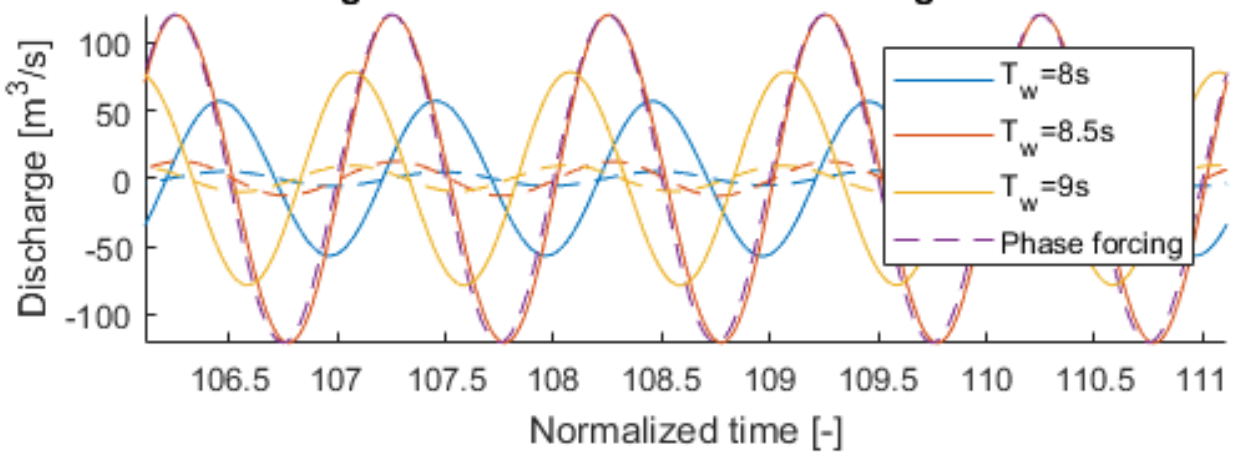

Figure 5.5: Time domain results damped system without weir flow 

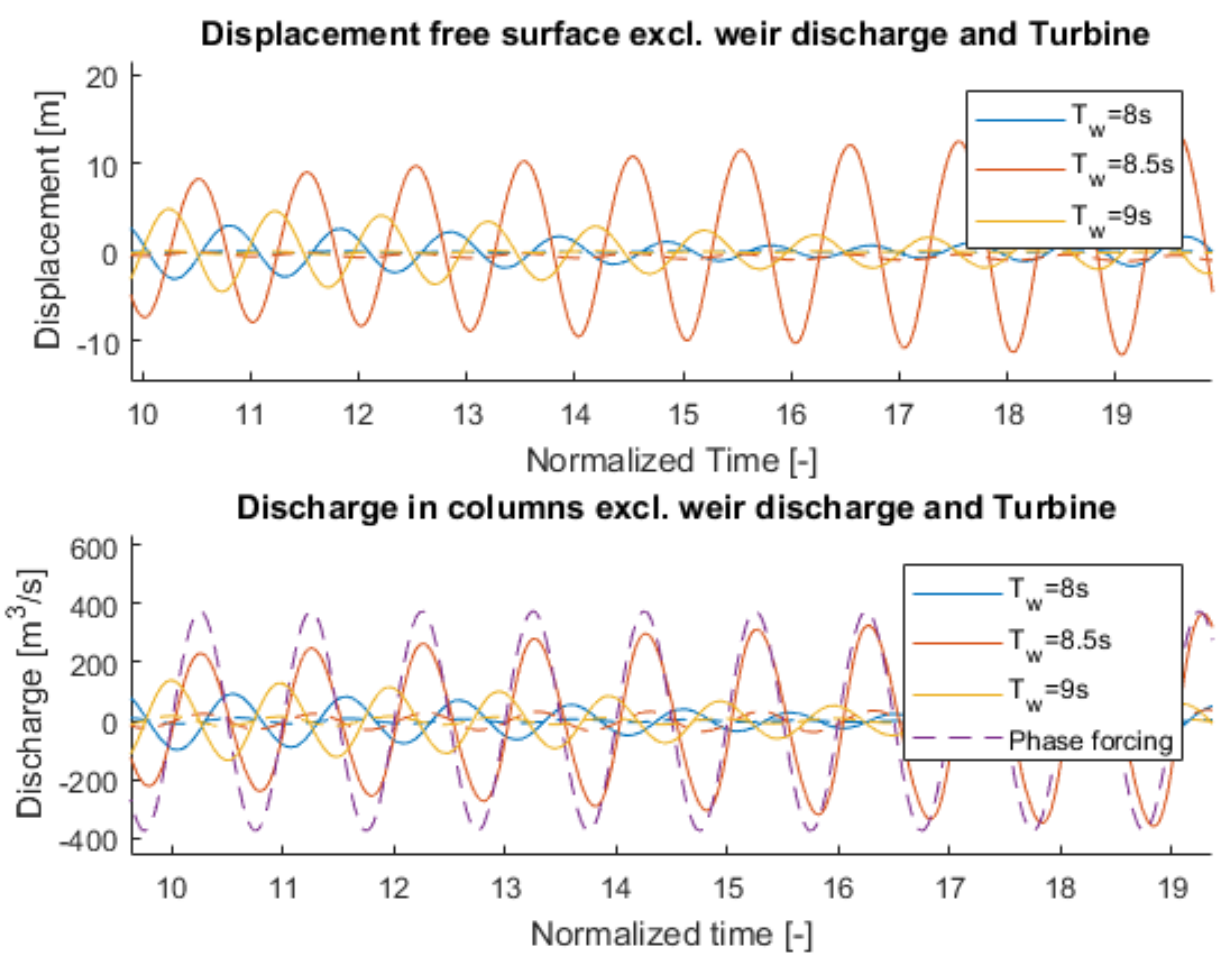

Figure 5.6: Time domain results undamped system without weir flow start of the simulation

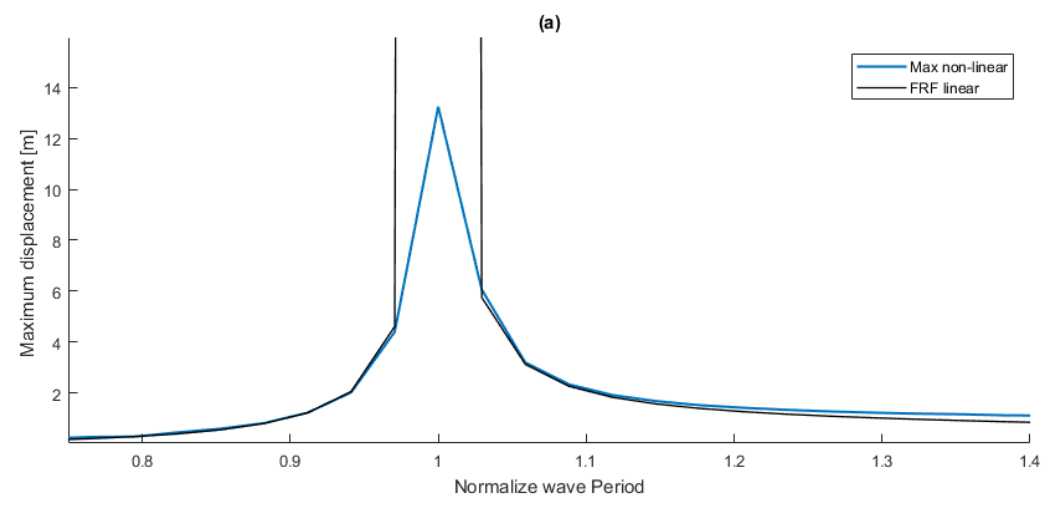

Figure 5.7: Comparison non-linear undamped system with linear frequency response function,

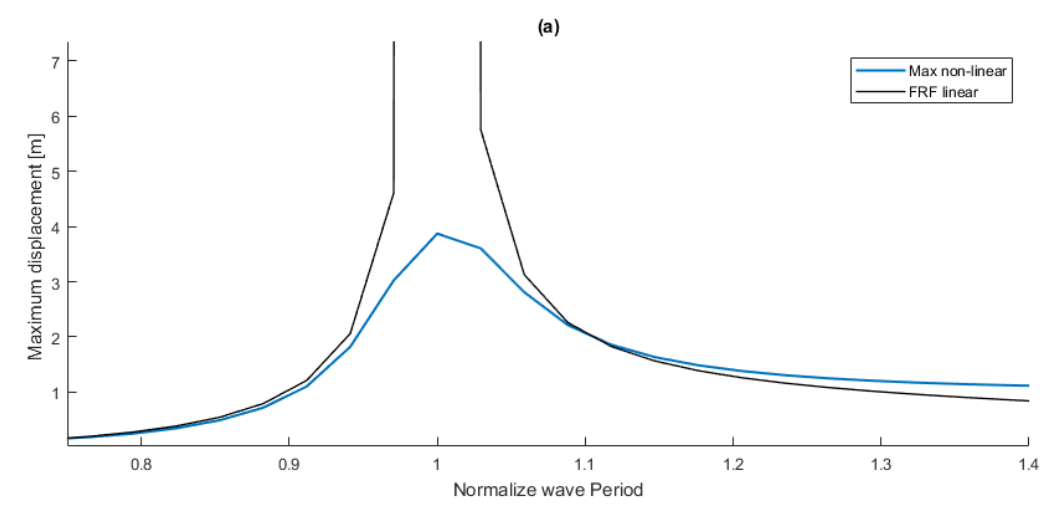

Figure 5.8: Comparison non-linear damped system with linear frequency response function 


\subsubsection{Weir discharge}

In the sections above, excluding the weir discharge, the behaviour of the non-linear simulations was found to be in line with the expected behaviour of such a system and could be approximated by the linear equations of motions fairly well, outside the region of the natural period. Additionally, the results of the damped system are found to be much more realistic and are therefore used only in the following section.

First, the overall effect of the weir discharge will be based on the maximum, minimum and mean responses of both columns. Secondly, the influences of the forces related explicitly to the mass transfer are presented; namely the pressure of the falling water, the change in pressures at the free surface.

\section{Maximum and minimum response}

In figure 5.9 the maximum displacement and discharge of both columns, the first column is represented by the solid lines and second column by the dashed lines. The minimum displacement and discharge are presented. The extreme values are obtained from the steady-state response at $t \gg 0$ of the time series. As seen in the figures, the simulations are run for multiple weir levels and a wide range of excitation periods. The latter is normalised with respect to the natural frequency of the system, $T_{n}=8.5 \mathrm{~s}$.

In the first column, the weir level is visible in the maximum displacement. The weir level is only exceeded for a weir level of $z_{w}=0.5 \mathrm{~m}$. For this weir level, the maximum displacement of the second column also exceeds the weir level, resulting in a simultaneous increase of the free-surfaces of both columns.

Compared to the results excluding the weir discharge, the maximum and minimum discharge and minimum displacement of the first column also decreases. The transfer of potential energy by the weir discharge reduces the amplitudes of the response, concluded from the higher reduction in discharge for lower weir levels.

In the second column, a small response was observed for the simulations excluding the weir discharge. However, the weir discharge causes the free-surface level to increase; this additional water in the second column is then to be discharged. The discharge is visible in the discharge of the second column. The discharge in the second $Q_{2}$ column oscillates between $Q_{2}=12$ and $-12 \frac{\mathrm{m}^{3}}{\mathrm{~s}}$ in the simulation the simulation without weir discharge. The maximum and minimum discharge are both negative for certain wave periods, indicating the desired single directional flow out of the second column.

For the weir level of $z_{w}=0.5 \mathrm{~m}$, the difference between the minimum and maximum are decreased from $\Delta Q_{2}=24 \frac{\mathrm{m}^{3}}{\mathrm{~s}}$ to $\Delta Q_{2}=13 \frac{\mathrm{m}^{3}}{\mathrm{~s}}$ compared to the discharge without weir discharge. The reduced discharge amplitude can be explained by; the high velocities occur around the same time of the discharge of the chamber, simultaneous to the high pressure in the chamber. Including the weir discharge, the air pressures are almost the same; however, the increased velocities increase the head losses, resisting the acceleration from the air pressure. Additionally, the increase free-surface level of the second column during the low air pressure cycle acts as an additional force to the second column, also reducing the acceleration. Both principles reduce the acceleration during the pressure oscillations resulting in the smaller difference between the maximum and minimum discharge.
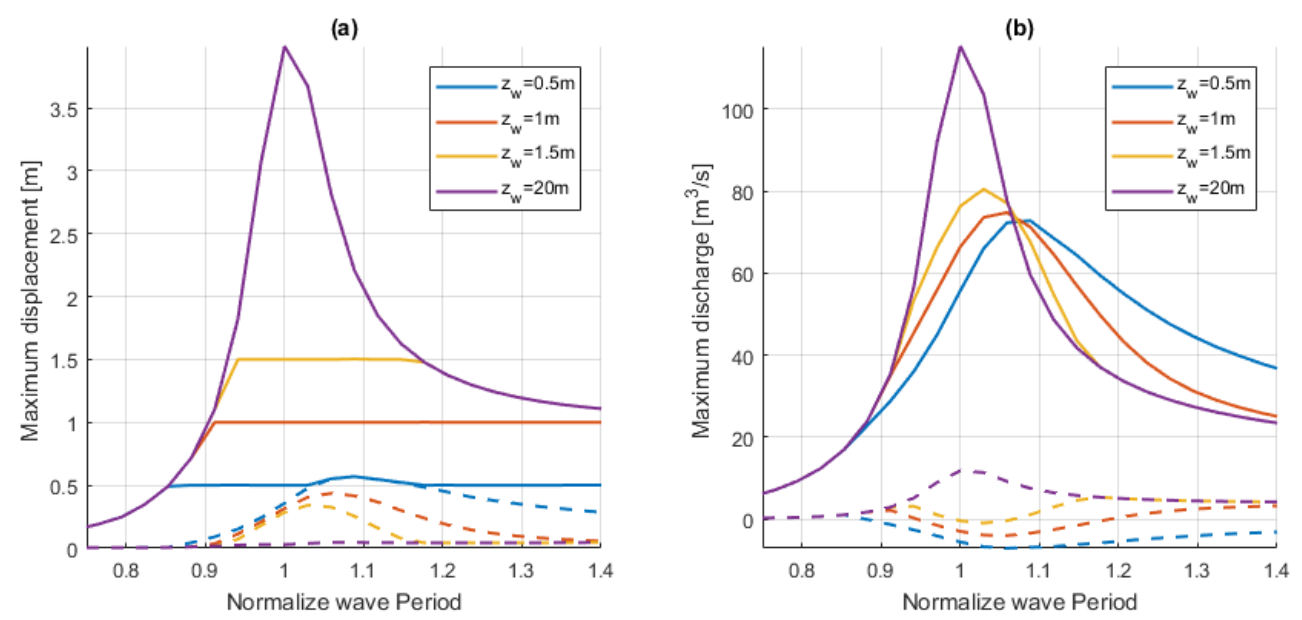

Figure 5.9: Maximum displacement and discharge of Column 1(solid lines) and Column 2 (dashed line) for different weir levels 

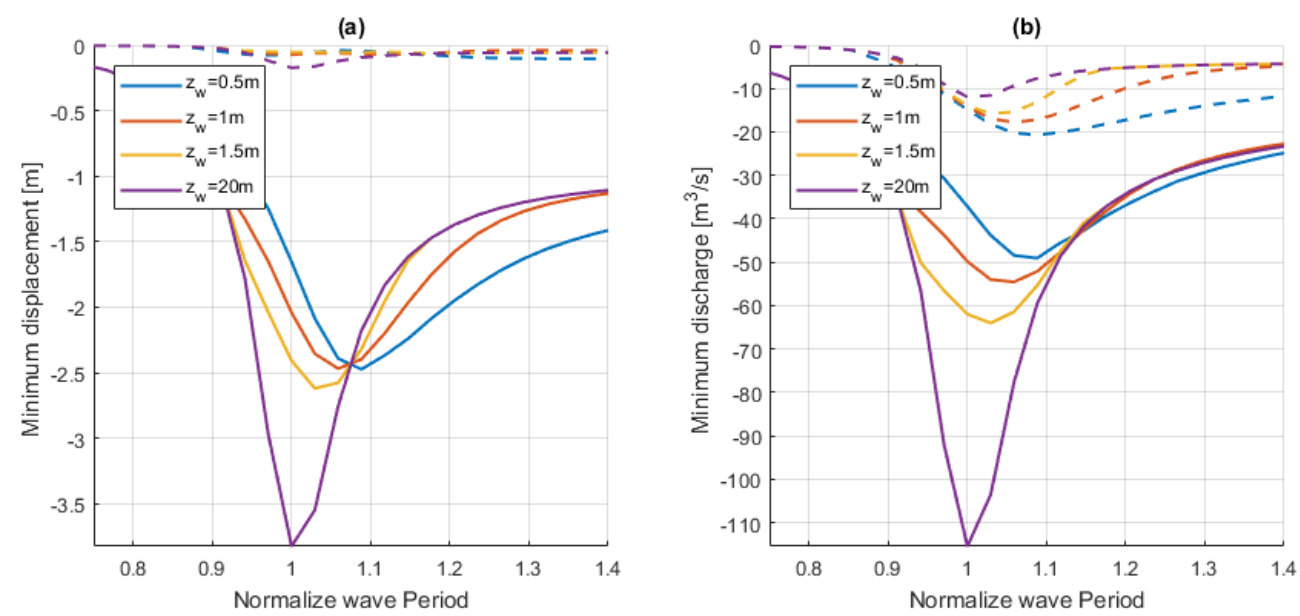

Figure 5.10: Minimum displacement and discharge of Column 1(solid lines) and Column 2 (dashed line) for different weir levels

\section{Mean response}

From the continuity of mass can be concluded that the water that enters the columns should also exit the system. On this can be agreed on by comparing the mean discharge of both columns with each other, presented in figure 5.11. In this figure the found mean discharges are exactly opposite to each other. Therefore it can be concluded that the mass transfer is modelled correctly.

Secondly, the mean discharge of both columns is directly related to the mean weir discharge. The zero mean discharge for $z_{w}=20 \mathrm{~m}$ in both columns agrees with the no weir discharge situation.

Apart from the observations regarding the mean discharge, some interesting features are visible from the mean displacements of both columns.

The first thing that draws the attention is the increased mean displacement of the first column and the decreased value in the second column for the simulation without weir flow. Caused by the same stiffening and softening spring relation described earlier in this chapter. Because of the higher positive air pressure than the negative pressures decreases the mean displacement of the second column. Then for the equilibrium to be balanced, the mean displacement of the first column automatically increases.

In the weir discharge cases, this relation is reversed. With positive mean displacement in the second column and negative mean displacement in the first column. This is again caused by the balance equilibrium of the air pressure between the two columns. However, now instead of a positive mean air pressure on the second column, the free-surface level is increased by the additional water transferred from the first column.

The reduction in mean displacement also caused the weir discharge to increase less than expected for lower weir levels. This is mainly caused by the resistance from the pressure losses. To explain this, it is interesting to quickly return to the undamped simulations, the mean displacement and discharge of the undamped simulation if presented in figure 5.12. In this figure, the mean displacements are closer to zero compared to the damped case. Additionally, the discharge of $z_{w}=0.5$ is approximately two times higher than the discharge of $z_{w}=1 \mathrm{~m}$. While this factor is only 1.25 in the damped case. An additional cause of the reduction of the weir discharge is a slight reduction of the total discharge amplitude in the first column for lower weir levels, seen in figure 5.9 and 5.10 .

Concluded from this is that the flow resistance in the second column, caused by the head losses, increases the mean displacement of the column. Which, subsequently leads to a reduction of mean displacement of the first column due to the coupling by the air pressure. Finally, the lowered mean displacement of the first column results in a relative reduction in weir discharges, for the almost the same oscillation amplitudes in the first column.

\subsubsection{Period of the maximum response}

In addition to the observations regarding the maximum, minimum and mean values, a shift in the period of the maximum response is observed relative to its natural period. From the comparison between the mean discharge of the damped and undamped simulations, it is evident that the shift is related to the weir discharge. Further, in the equation for the natural period, the mass is found in the nominator of the square root and the stiffness in the denominator. From the mass transfer can be concluded that the mass in the 

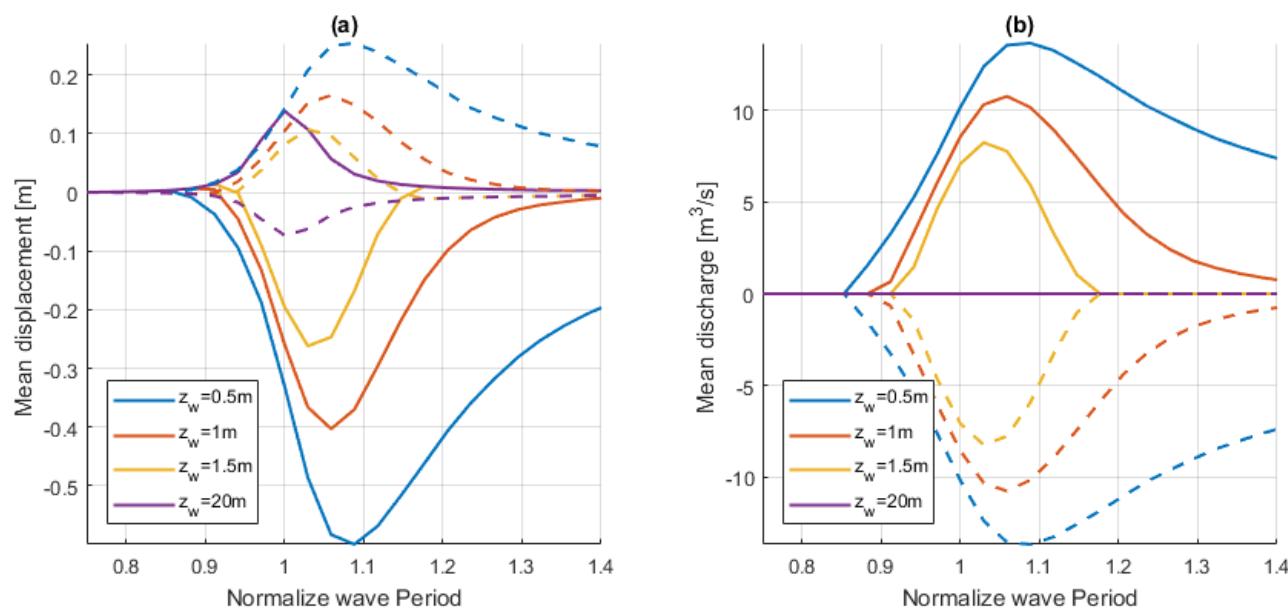

Figure 5.11: Mean displacement and discharge of Column 1(solid lines) and Column 2 (dashed line) for different weir levels

(a)

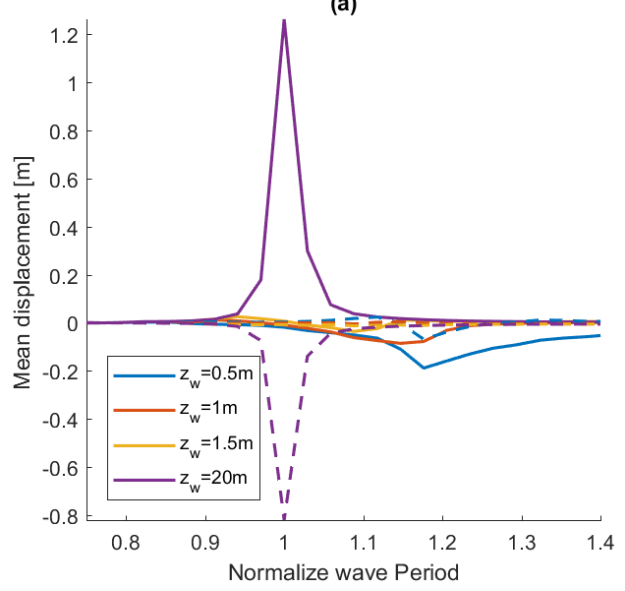

(b)

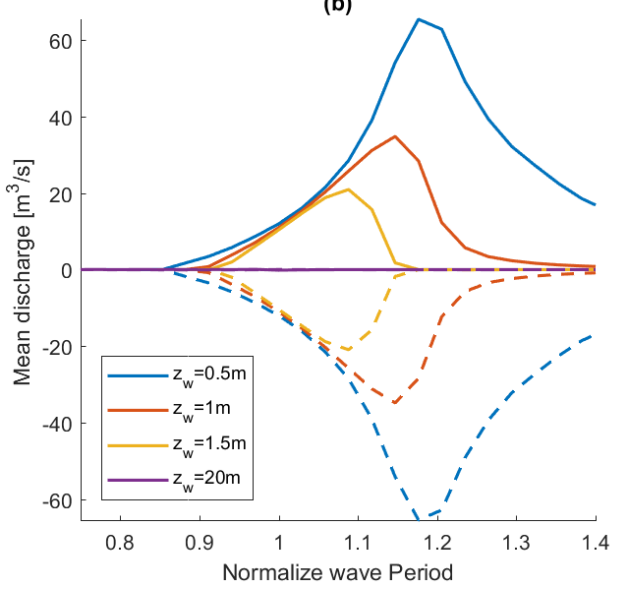

Figure 5.12: Mean displacement and discharge of undamped model of Column 1(solid lines) and Column 2 (dashed line) for different weir levels

resonating column doesn't increase during the discharge in the second column. The increasing period must, therefore, be related to a decrease in stiffness. From the total continuity of mass equation and the incompressible fluid, the air pressure restoring force can be taken constant, the stiffness reduction must, therefore, be initiated by the gravitational restoring force. In standard oscillating bodies all kinetic, excluding damping, is transferred into potential giving an opposite force on the column, which is maximum at the maximum and minimum displacements. Here, however, a part of the free surface level doesn't rise above the weir level, and the water flows away. Now not all kinetic energy in the resonating column is transferred to potential energy. The reduced opposing force results in a slower response of the column with respect to the forcing. In figure 5.13, presenting the time series of the damped response for a weir level of $z_{w}=0.5 \mathrm{~m}$ and three excitation periods, this can be seen in the oscillations of the velocity.

Comparing the maximum and minimum peak of the velocity to the forcing is can be seen that the minimum peaks are shifted to a bit later in time than the maximum values. The origin of this shift is the reduced steepness of the reducing velocity during weir discharge, which can be seen by looking at the graphs carefully. In the graph, it now can be seen that phase of the response the excitation period equal to the natural period $T_{w}=T_{n}=8.5 \mathrm{~s}$ is lagging behind the forcing. The phase of the 9-second oscillation is now nearly in phase with the forcing. Previously this the phase was found to be beforehand the forcing.

Additionally, the relative difference between the amplitude for different periods is also found to be smaller compared to the non-weir discharge case. This results in more significant responses for higher periods, increasing the bandwidth of the system. 

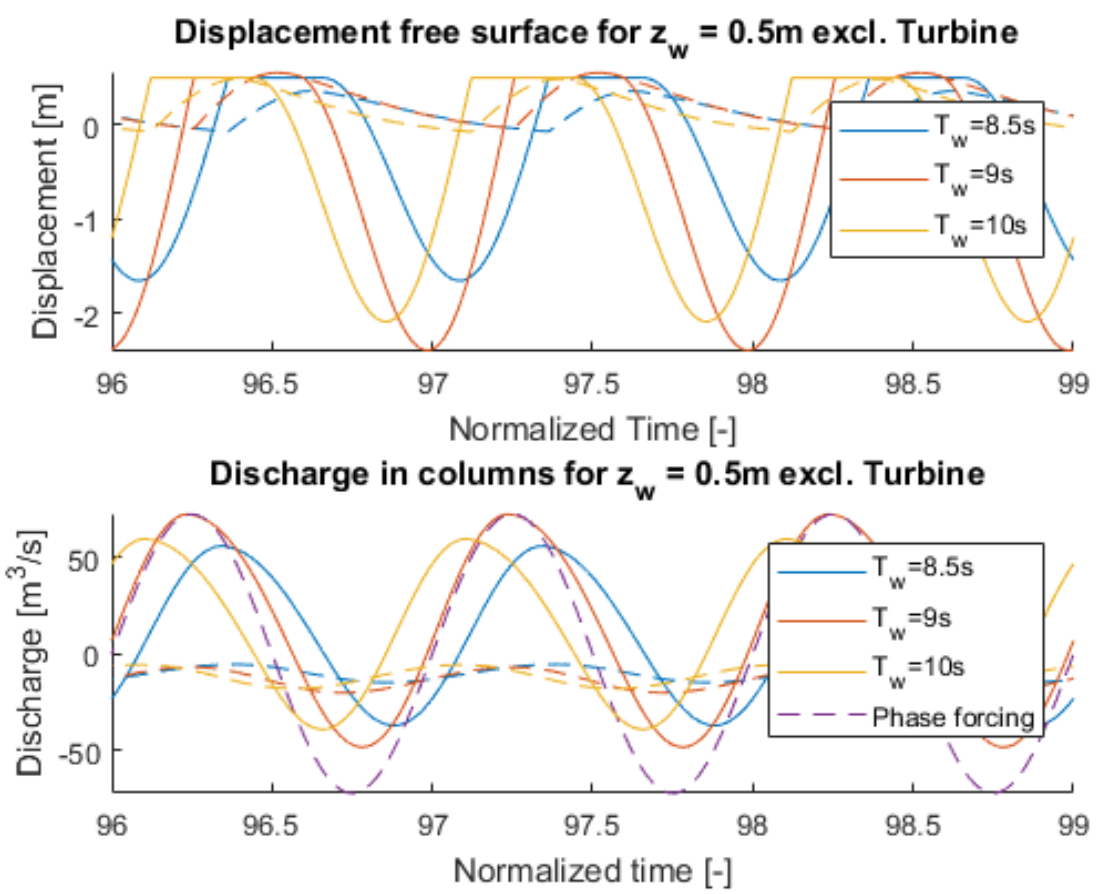

Figure 5.13: Damped time domain steady state response for a wave amplitude of $2 \mathrm{~m}$

\section{Pressures associated with the weir discharge}

In this section, the pressures related to the weir discharge are given, namely the:

- The falling water

- Change in convective pressure

- Change in mass change momentum

The compared results are obtained for a weir level of $z_{w}=0.5 \mathrm{~m}$ and a wave amplitude of again 2 meters. The amplitude of the wave pressure and air pressures at given in figure 5.14. From the graphs, pressures in the order of $10^{4} \mathrm{~Pa}$ are found.

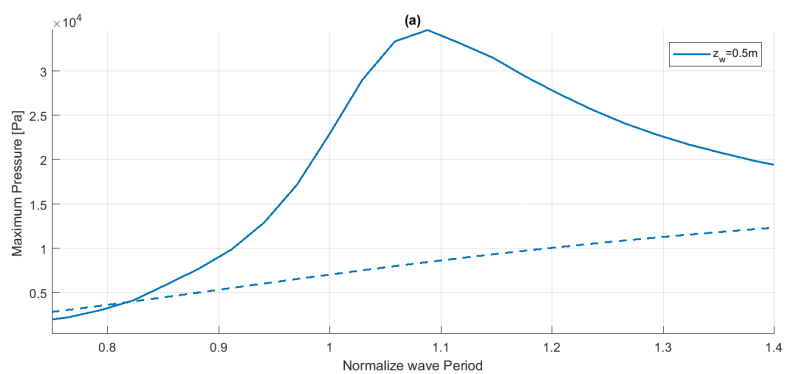

Figure 5.14: Maximum air pressure (solid line) and maximum wave pressure on column 1 (Dashed line)

In table 5.1, the maximum and mean values of the pressure related to the weir discharge are given at the resonance periods of the system. The values for the convective and mass change pressure are obtained for:

$$
\begin{gathered}
P_{\text {conv }}=\frac{1}{2} \rho\left(\frac{Q_{w}}{A_{c}}\right)^{2} \\
P_{\text {conv }}=\frac{A_{c} x}{V_{c}} \rho\left(\frac{Q_{w}}{A_{c}}\right)^{2}
\end{gathered}
$$


Table 5.1: The maximum and minimum pressures related to the weir discharge in $P a$

\begin{tabular}{|c|c|c|}
\hline Pressure type & Maximum & Mean \\
\hline Fall & -1400 & -160 \\
\hline Convective column 1 & -1672 & -250 \\
\hline Convective column 2 & 100 & 10 \\
\hline Mass change column 1 & -45 & -6 \\
\hline Mass change column 2 & 2 & 0.2 \\
\hline
\end{tabular}

From the table, it can be seen that pressure related to the change of mass in the columns only are minimal. Additionally, the large area and the small velocities of the second column results in low pressures related to the convective mass in this column.

For the impact pressure and convective pressure, much higher maximum pressures have been found. The mean pressures are, however, much smaller due to the short duration of the weir discharge.

Regarding these pressures, it is important to note that the maximum pressure occurs for the higher velocities of the first column. Over the duration of the weir discharge, this high velocities deduces to zero, reducing the pressure directly.

For the response of the second column, the direction of the impact pressure is in the same direction as the air pressure. The impact will give an additional acceleration to the outgoing flow, aiding the dynamics of the second column. This further acceleration does, however, also results in higher peak velocities in the second column. These peak velocities lower the efficiency of the turbine. The impact pressure could, therefore, be undesirable. However, the total effect of the impact pressure is assumed to be small, because; the inertia is very high, the dynamic pressure in the small diameter exit duct, the impact pressure decreases to zero during the weir discharge and the rigid body approach used in this research do not comply with the assumption of the impact to be spread over the full area of the column.

Also, the convective pressure in the first column is assumed to have a relatively small effect on the dynamics. In comparison to the total air and wave pressure of the pressure is very small. Additionally, the hydrostatic pressure at the weir level $z_{w}=0.5 \mathrm{~m}$ is $p_{x_{1}}=5000 \mathrm{~Pa}$ and thus more than four times larger than the convective pressure. The small effect that this pressure will have on the system is a reduction in the non-linear restoring force. Namely, the convective pressure without weir discharge gives a pressure opposing to the flow, the weir discharge reduces this opposing pressure and will thus decrease the stiffness.

Concluded from this analysis above is that the pressures related to the weir discharge have a limited effect on the total behaviour of the system caused by:

- The velocity of the resonating column reducing to zero during the weir discharge.

- The high air chamber and wave pressures

- The large area of the second column

The difference in response between the simulation, including and excluding weir discharge is thus governed by the actual mass transfer, pumping mechanism, instead of the pressures related to this mass transfer.

\subsection{Response including power take-off}

After the analysis of the response excluding the power take-off, the response including it is analysed in the following sections. First, the general effect of the power take-off is evaluated. After that, the impact of different turbine diameters is , and the optimal dimension for the present system is given. Lastly, the efficiency of the power take-off is compared for various wave heights and weir levels.

\subsubsection{Turbine response}

In this section, the effect of the power take-off of the turbine on the behaviour of both columns is presented. The response is simulated for various rotation speeds of the turbine. In figure 3.7 in chapter 3 it was found that the way op modelling overestimated the power capacity of the turbine for velocities above the limit velocity, this will induce additional damping to the system and reduce its response. This is caused by the modelling limitation of the constant speeds. However, the increased turbine damping doesn't have to mean that the response of the system is completely dampened out. Therefore the results for simulations with velocities at 


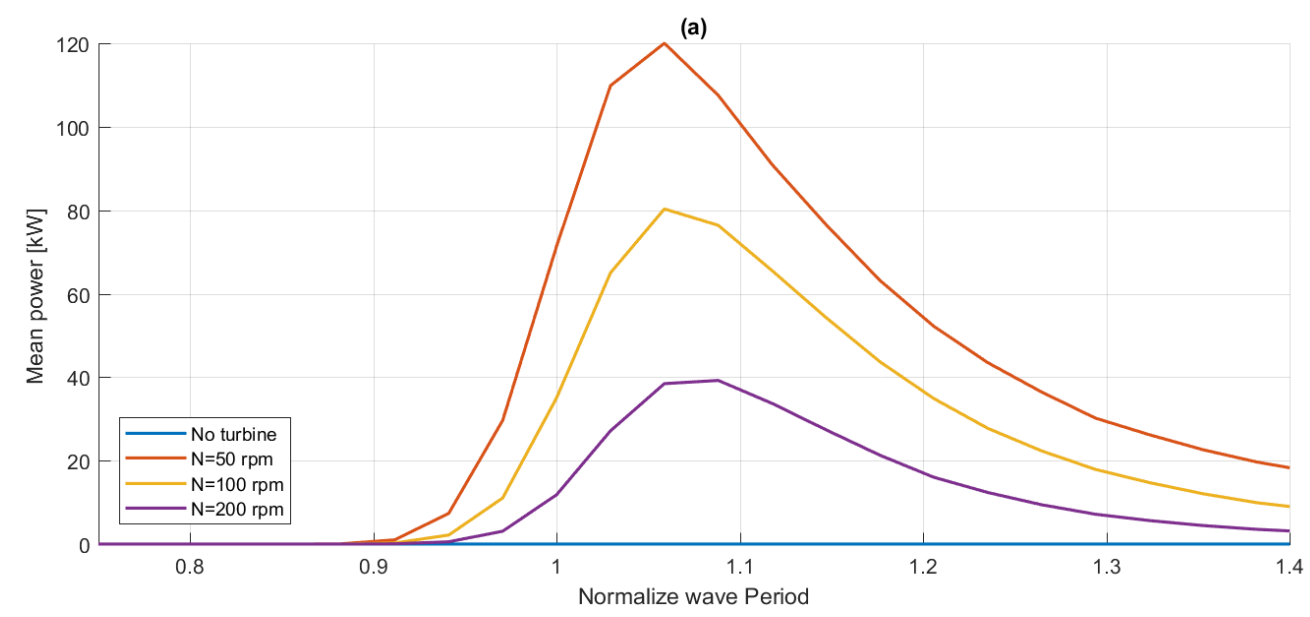

Figure 5.15: Mean power for different rotation speeds for $D_{t}=2 \mathrm{~m}$

the turbine higher than the limit velocity, given in appendix A, will be used in the analysis. The requirement for assuming 'valid' results for this case is that the flow in at the turbine is uni-directional.

In figure 5.15, the mean power take-off is presented for different rotation speeds and a weir level of $z_{w}=$ 0.5 and a wave amplitude of $a_{2}=2 \mathrm{~m}$. In this figure, it can be seen that a low rotation speed is capable of generating the most energy. For this speed, the turbine is more efficient at lower flow velocities and is thus capable of generating a higher average power.

In figure 5.16 is seen that this higher power take-off reduces the mean velocity at the turbine. This also means that the weir discharge is diminished. The cause of this reduction can be explained by the increased damping as seen in the comparison between the damped and undamped simulation, excluding weir discharge. Here the same change in mean displacements was observed causing the reduces discharge. As expected, the reduced discharge also shifts the peaks of the responses closer to its natural frequency.

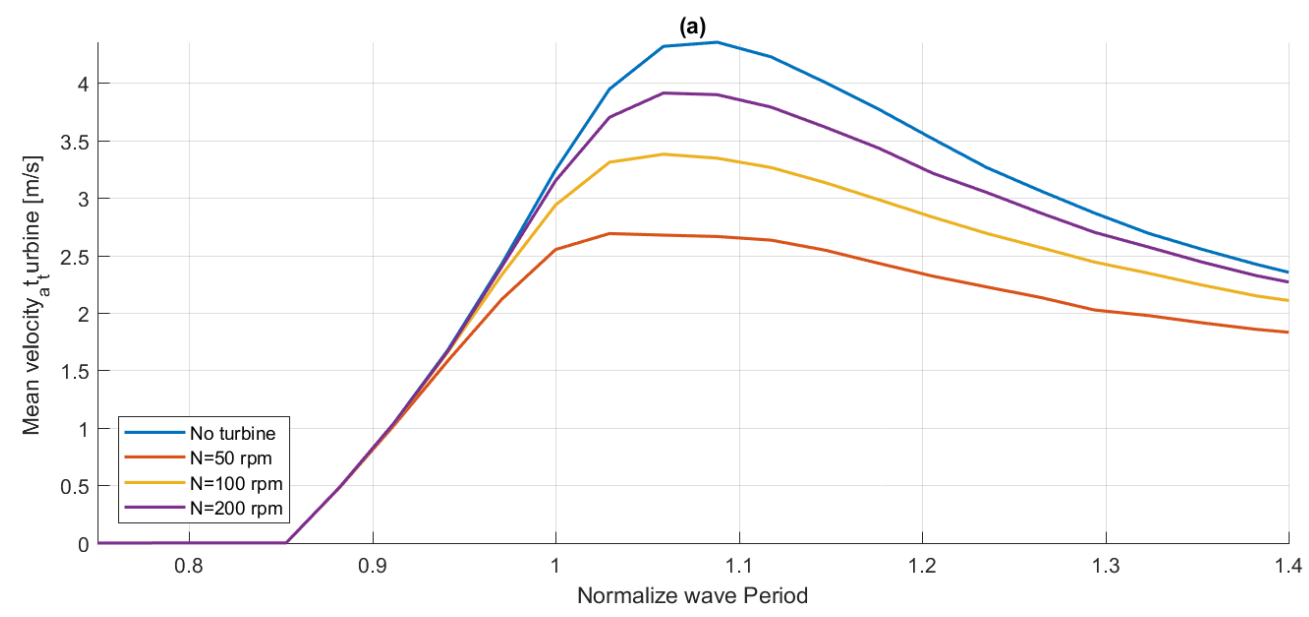

Figure 5.16: Mean velocity at turbine for different rotation speeds

Further, from the maximum and minimum velocities at the turbine change in shape observed, figure 5.17 and 5.18. From the results, it can be seen that the amplitude of the velocity remains approximately the same for different rotation speeds. However the reduced mean velocity goes together with a flatter peak, this flatter peak results in a local trough at the minimum velocity at $N=50 \mathrm{r} \mathrm{m}$. Increasing the damping will eventually result in the trough to get below zero, indicating a reversed flow during the oscillations. As described above, for the latter phenomena the results will be assumed invalid.

From the velocity and power graphs is further observed that; the differences between different rotation speeds is the highest around the natural periods, are equal for low periods and the results converge for higher periods. 


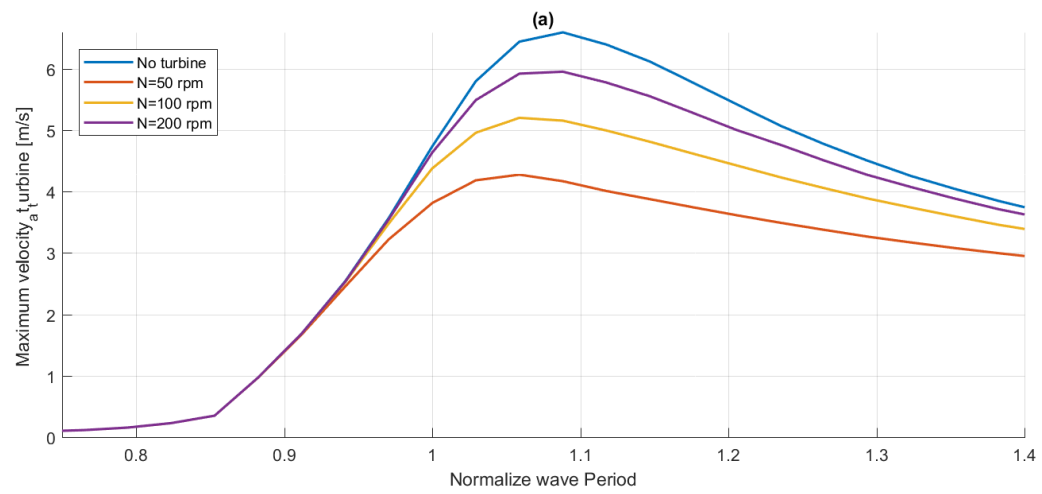

Figure 5.17: Maximum velocity at turbine for different rotation speeds

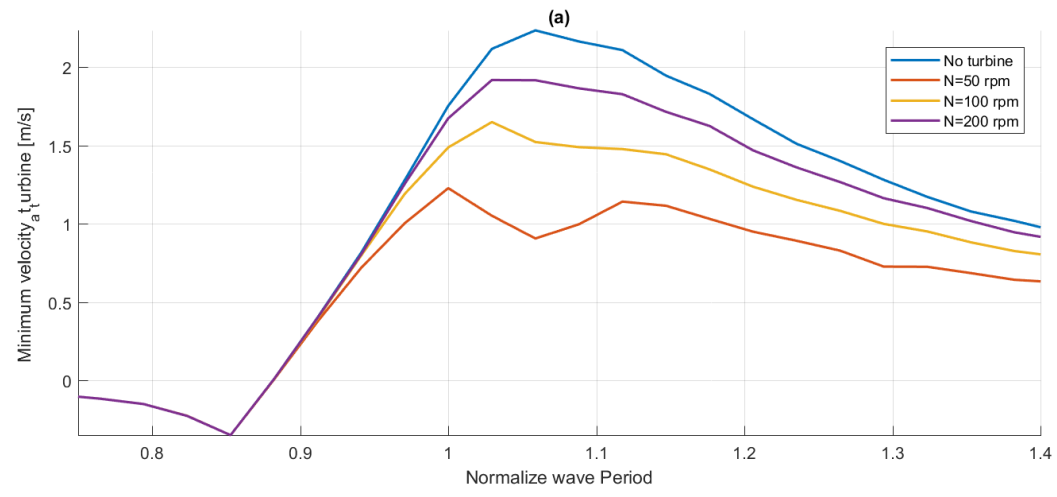

Figure 5.18: Minimum velocity at turbine for different rotation speeds

Apart from the changed response in the second column, some interesting results are found in the first column. Namely, due to the decrease in weir discharge the maximum discharge in the first column increases for higher energy extraction, as shown in figure 5.19. Due to the reduced transfer of potential energy to the second column, the oscillation amplitudes increase for the same excitation forces. This increase thus results in higher maximum velocities despite the lower mean velocity. The energy dissipated by the pressure losses does however, also increase for the increasing velocities.

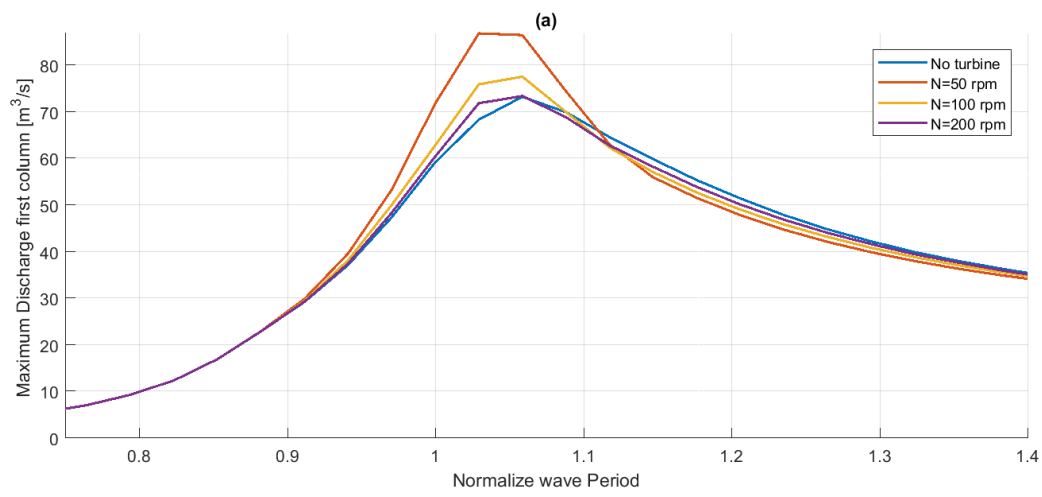

Figure 5.19: Maximum discharge first column for different rotation speeds 


\subsubsection{Turbine diameter}

In the following part, the simulations are run for different turbine diameters. For a set length of the exit pipe, the inertia of the second column is supposed to change together with the diameter. To avoid this parameter to influence the response and affect the comparison, the equivalent length, described in equation (3.77), is kept the same. Now to determine the effect of the diameter to the response and find its optimal dimensions, the mean generated powers are presented for each turbine rotation speed. In the simulations again, a wave amplitude of 2 meters is used and a weir level of $z_{w}=0.5 \mathrm{~m}$.

From equation (3.82) the power performance of the turbine decreases for an increasing diameter proportional to $P_{t u r} \propto\left(\left(\frac{1}{D_{t}^{2}}\right)^{k 2}\right)^{2}$. Additionally the velocity at the turbine is also dependent on the area of the turbine squared in the denominator, the dynamic pressure head in equation (3.81) in related to the diameter proportional to $H \propto\left(\frac{1}{D_{t}^{2}}\right)^{2}$. By combining both equation it can be seen that an increase diameter will therefore decrease the energy take-off for the same discharge.

The mean power take-off for turbine diameter between the $D_{t}=1.5-2.5$ meter are presented in figure $5.20,5.21,5.15,5.22$ and 5.23. The graphs with the column response are presented in appendix C, theses graphs are used to find the flow conditions in the second column and say if the results are valid for the regarding the modelling of the turbine.

The first thing that can be seen is the almost equal results for $N=50 \mathrm{r} \mathrm{pm}$ and $N=100 \mathrm{r} \mathrm{pm}$ for $D_{t}=1.5 \mathrm{~m}$. For the small diameter, the velocities are higher for the same discharge. This increases the energy extraction are lower discharges dampening the responses, for both rotation speeds the system is highly damped, and there is not more energy available to extract power. It is resulting in the closely similar lines in the power curve. The flow direction for both speeds 50 and $100 \mathrm{rpm}$ are found to be reversed.

For increasing diameters, the distance between the lines for different rotation speeds of the power extraction increase. Resulting in a lower power take-off by for large diameters and high rotational speeds.

From the various diameters, it is seen that the highest powers are generated for the diameters of 1.75 and 2 meter. For the large diameters, this corresponds to the statement of; the power being proportional to the diameter. However, for $D_{t}=1.5 \mathrm{~m}$ the power take-off was reduced compared to the larger diameters. Comparing the mean displacements of the different diameters the mean displacements of the second column were found more substantial for the smaller diameters, also in the simulation excluding power take off. This is caused by the higher velocities and increased resistance from the pressure losses. From this lower discharge, it is clear that a smaller amount of energy can be extracted.

Keeping the decreasing mean displacements for larger diameters the discharge therefore also increases for larger diameters. This is visible looking at the mean velocities at the turbine, these are almost the same for the diameters $2.5 \mathrm{~m}$ and 2.25 meter. From $D_{t}=2 \mathrm{~m}$. The mean velocity decreases despite the decrease in velocity. For larger diameters we can now say that the discharge increases. However, the larger diameter also reduces the performance for the same flow for its proportionally on the diameter.

Now with the velocities of $D_{t}=2,2.25$ and $2.5 \mathrm{~m}$ almost the same, the pressure losses are equal. The reduction in discharge in the steady state is therefore mostly related to the resistance caused by the power take-off. Which is higher for $D_{t}=2 \mathrm{~m}$. For $D_{t}=1.75 \mathrm{~m}$ approximately the same power take-off is found; however, the reduced discharge causes the flow to be reversed at the turbine, at its resonance period, this result is therefore considered invalid. Concluded from these observations is that the optimal power take-off is found for a turbine diameter of $D_{t}=2 \mathrm{~m}$ with rotation speed $N=50 \mathrm{r} \mathrm{pm}$. This amount of power is assumed to be the limit of this system, where higher resistances reduce the discharge even more, decreasing the power take-off, and larger diameters are simply less efficient for the same flows. 


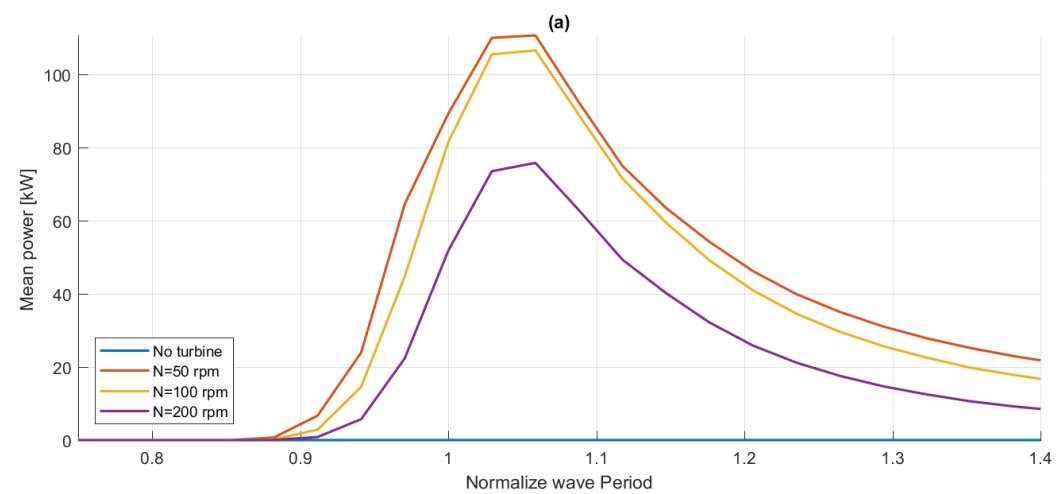

Figure 5.20: Mean power for different rotation speeds for $D_{t}=1.5 \mathrm{~m}$

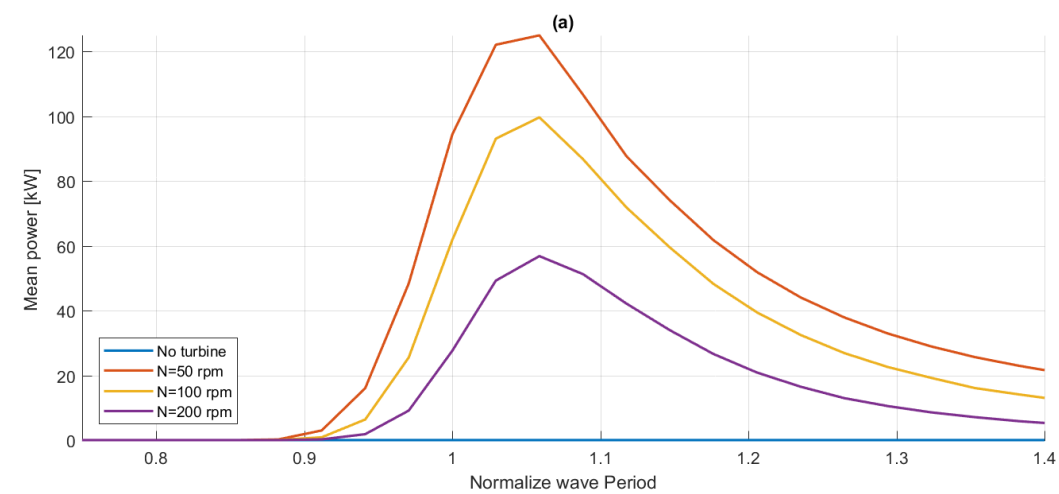

Figure 5.21: Mean power for different rotation speeds for $D_{t}=1.75 \mathrm{~m}$

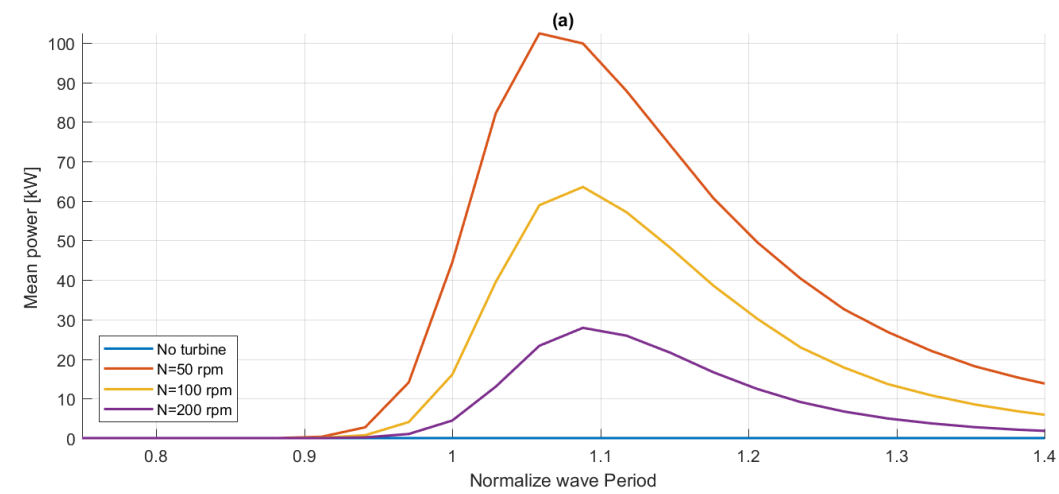

Figure 5.22: Mean power for different rotation speeds for $D_{t}=2.25 \mathrm{~m}$

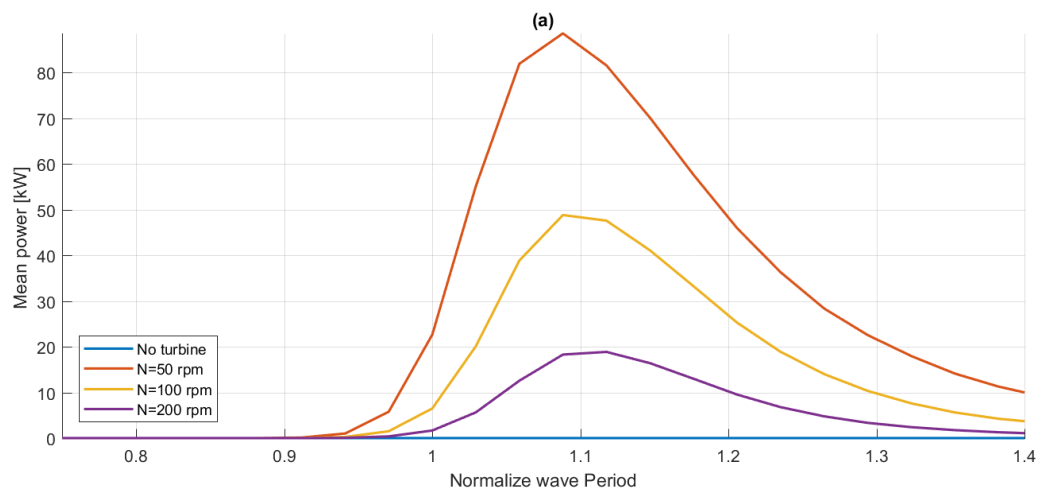

Figure 5.23: Mean power for different rotation speeds for $D_{t}=2.5 \mathrm{~m}$ 


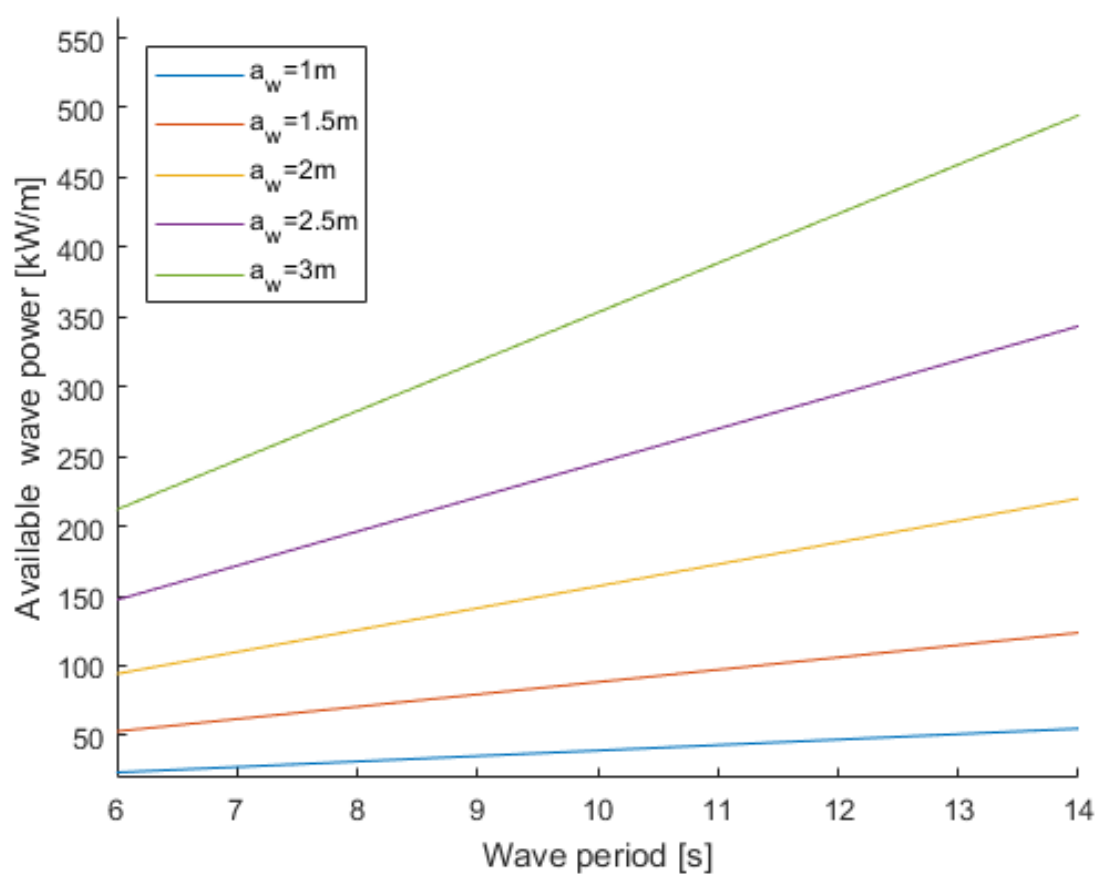

Figure 5.24: Available power per meter wave crest of regular incoming waves with different amplitudes

\subsubsection{Wave height}

In the following section, the influence on the wave weight on the power response of the system. The pressure at the mouth has a linear relation concerning the wave height. The maximum response of the resonating column is therefore also assumed to behave linearly with respect to the wave heights. However, this will not be the case for the power take-off because it is dependent on the weir discharges, which was found to be highly non-linear. Therefore this section will estimate the performance to different wave heights for different weir levels. As found in the analysis regarding the turbine, it is found that the most energy was captured at a low rotation speed. Therefore the results presented in this chapter are obtained for a rotation speed of $50 \mathrm{r} \mathrm{pm}$ and a turbine diameter of $D_{t}=2 \mathrm{~m}$, which was found to obtain the best results. Additionally, the performance will be assessed for its efficiency instead of the total power, to be able to compare the performance for the various wave heights. The available power for each simulated regular wave was found in equation (2.7); the results for the analysed wave amplitudes are shown in figure 5.24.

The efficiencies for different wave amplitudes at the same weir level differ from each other, was seen in figure 5.25 for a weir level of $z_{w}=0.5 \mathrm{~m}$. Now comparing the results for different weir levels, in figures 5.26, 5.27 and 5.28, it can be seen that the order of wave amplitudes with the highest efficiencies change.

For each wave amplitude, an optimum weir level can be found. These optimal levels are found for the five different waves simulated with weir level intervals of $\Delta z_{w}=0.1 \mathrm{~m}$. The results are presented in table 5.2. From these results, it is seen that the system is more efficient for lower amplitudes.

Additionally, from the figures and the table, it is seen that the lower amplitudes are more efficient for lower weir levels and the other way around. This can be explained by the fact that the response of smaller amplitudes is lower and therefore have a low discharge at high weir levels. On the other hand, for high amplitudes, the flows are much bigger and a low weir level would mean that the second column displacements exceed the weir level quickly. This way less water can be discharged into the second column. Additionally, The higher weir levels however, also increase the amount of kinetic energy remaining in the resonating column, and thus reducing the efficiency.

From the results, it can be concluded that an optimal weir level can be determined for each wave amplitude. The efficiency to simulated regular waves, with amplitude from $a_{w}=1-3$, is found to be between the 12 and $7.5 \%$. For lower amplitudes, it is expected that the efficiency will increase slightly and decrease for higher amplitudes. 


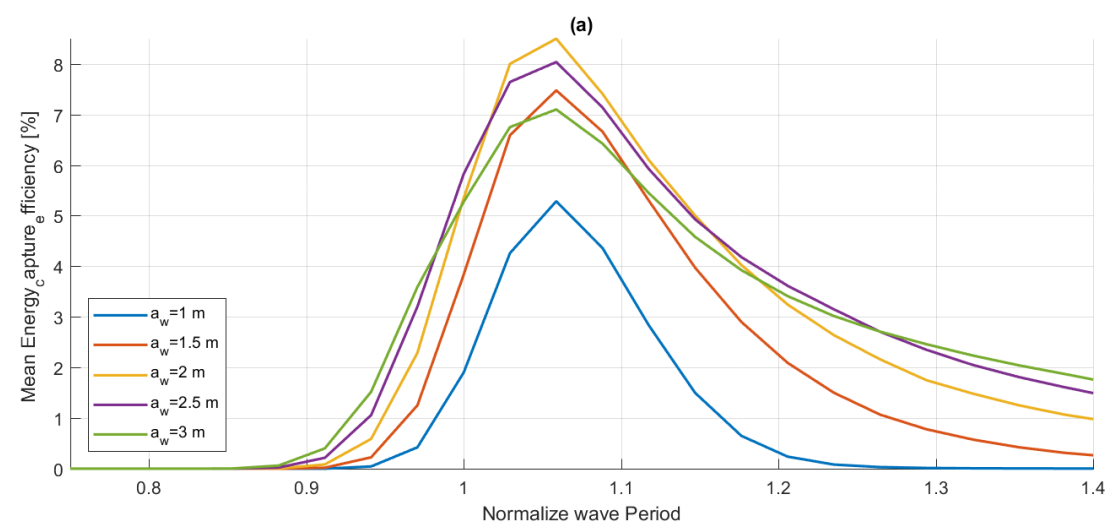

Figure 5.25: Power efficiency for varying wave amplitudes at a weir level of $z_{w}=0.5 \mathrm{~m}$

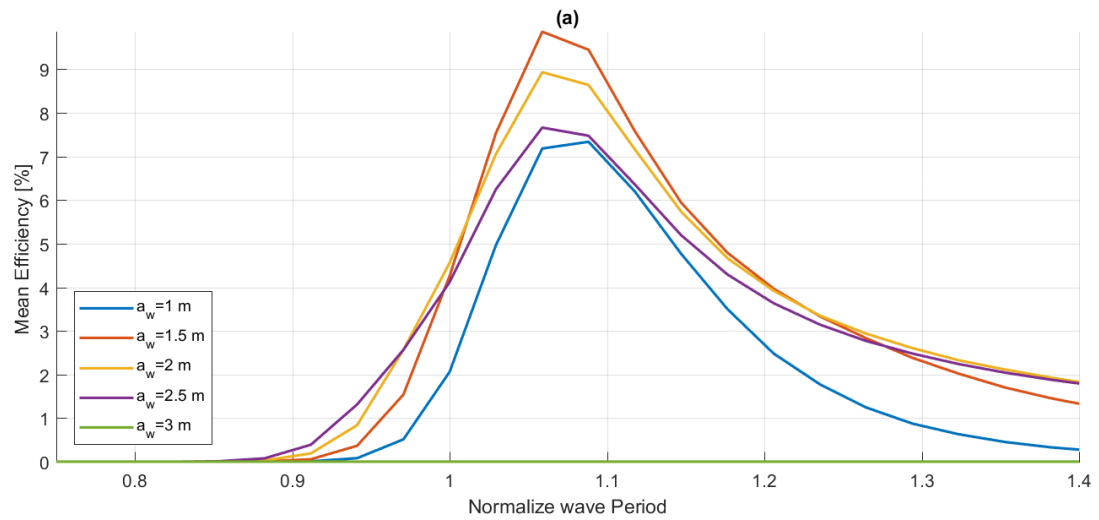

Figure 5.26: Power efficiency for varying wave amplitudes at a weir level of $z_{w}=0.3 m$

\subsection{Conclusion and Discussion}

In this chapter, the results of the simulations are presented. The non-linear equations of motion are solved in the time domain, from the resulting time series the extreme and mean values are obtained. This is repeated for regular incoming waves with different wave periods and parameter settings.

\subsubsection{Response excluding power take-off}

First, the results of simulations for the non-linear system excluding weir discharge were compared to the frequency response function of the linearised equations. The results are found to be in agreement with each other for periods away from the natural periods. Around natural period the damping related to the pressure losses was found to have a significant effect on the amplitude of the response.

Afterwards, the results were obtained including the weir discharge but excluding power take-off. The main observations in this analysis are:

- Due to the weir discharge the potential energy in the resonating column is transferred to the second column

Table 5.2: Maximum mean power output for different wave amplitudes and optimal weir level

\begin{tabular}{|c|c|c|c|}
\hline Wave amplitude [m] & Weir level [m] & Efficiency [\%] & Power [kW/m] \\
\hline 1 & 0.1 & 12 & 4.35 \\
\hline 1.5 & 0.2 & 10.6 & 7.84 \\
\hline 2 & 0.4 & 9.1 & 12.8 \\
\hline 2.5 & 0.5 & 8.0 & 17.7 \\
\hline 3 & 0.7 & 7.5 & 22.5 \\
\hline
\end{tabular}




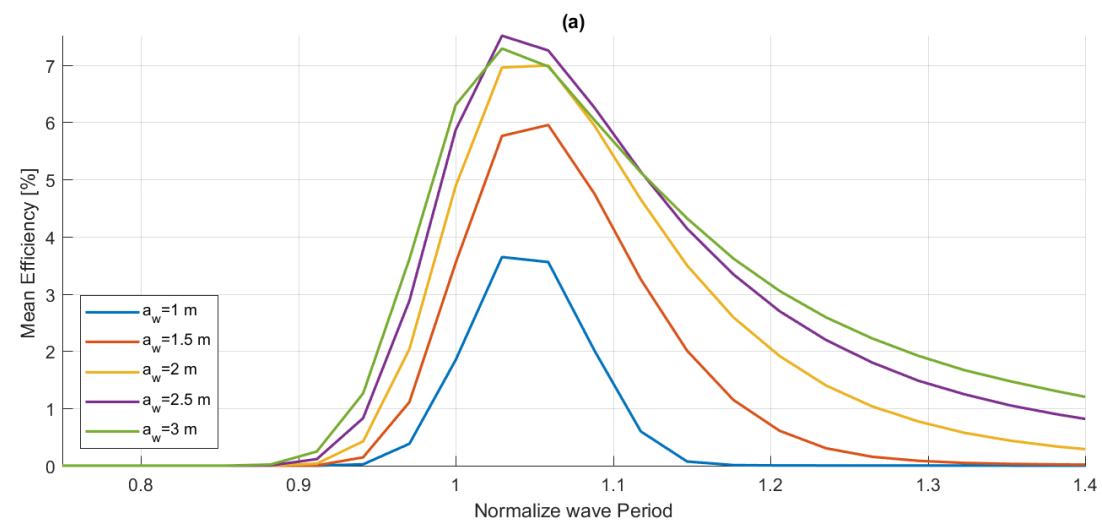

Figure 5.27: Power efficiency for varying wave amplitudes at a weir level of $z_{w}=0.7 \mathrm{~m}$

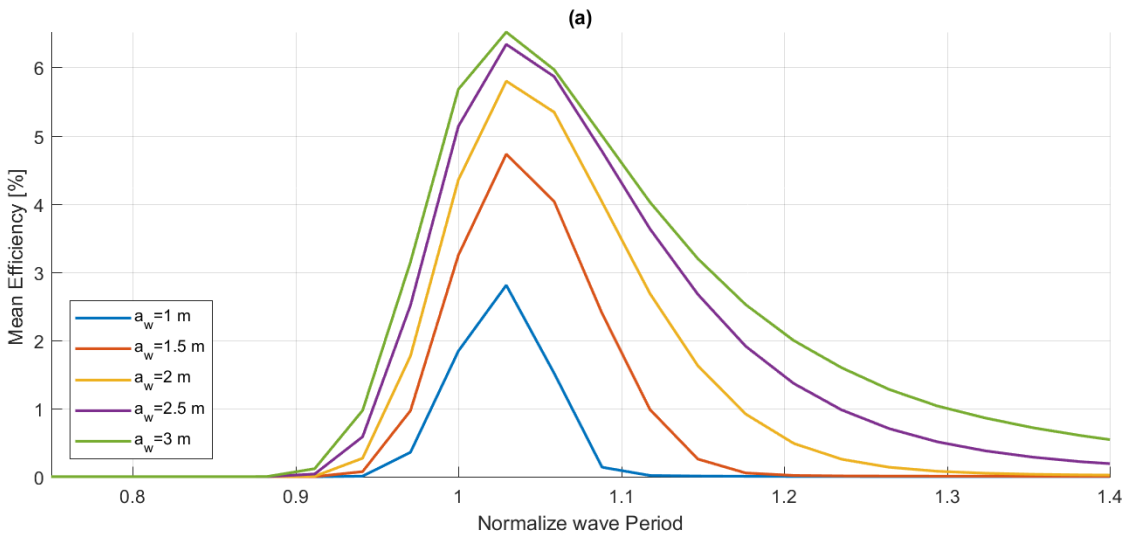

Figure 5.28: Power efficiency for varying wave amplitudes at a weir level of $z_{w}=1 \mathrm{~m}$

- The amplitude of the discharge is reduced together with the minimum displacement

- The phases of the response shift compared to the forcing during the weir discharge, giving a shift in the maximum response periods.

- The weir discharge increases the free-surface of the second column

- The weir discharge results in a pumping motion through the system

- A high weir discharge in combination with the high inertia results in a flow in a single direction.

- The additional water in the column reduces the oscillation amplitude of the flow in the second column.

- The pressure losses result in a decrease of weir discharge.

- Firstly, the additional resistance in the second column increases the mean displacement of the second column. Secondly, the air pressure coupling reduces the main displacement in the first column. This reduces the weir discharge for the same oscillation amplitudes in the first column.

- The peak periods are reduced by the lower weir discharge

- The response decreases slower for higher wave periods, increasing the bandwidth.

The impact pressure and the changes in momentum in both free surfaces were found to affect the behaviour only a little. The corresponding maximum pressures are small in comparison with the pressures of the air chamber and the waves. 


\subsubsection{Response including power take-off}

Next, the results were obtained for the simulations including the power take off. Due to the additional resistance of the turbine, the weir discharge reduces due to the increase of mean displacement of the second column, as seen in the damped simulation excluding power take-off. The reduced weir discharge reduces the peak period shift and the oscillating amplitudes in the first column increase. The latter also increases the pressure losses and will reduce its efficiency.

Despite the reduced discharge, the high turbine damping, corresponding to a rotation speed of $N=$ $50 \mathrm{rmm}$, did result in the highest average power take-off around the natural period of the system. In periods the response is found the same for different damping ratios of the turbine. For higher wave periods, the results were found to converge to the non-power take-off case because of the lower energy capture.

For the given wave forcing the different turbine, diameters were found to have a small but noticeable effect on the performance of the system. The modelled turbine power take-off was found to be less efficient for larger diameters for the same flow. In the response of the larger diameters, the lower turbine damping resulted in a higher mean weir discharge, but lower total power generations. The opposite was found for smaller diameters; here the higher damping increasingly reduces the weir discharge and the available power. The generated power also decreases. From these relations, an optimal turbine diameter of $D_{t}=2 \mathrm{~m}$ was found. This results yielded the heights power and a single directional flow for all excitation periods.

Lastly, the performance of the system was found for different incoming wave heights. The optimal is related to the weir level because the small wave amplitudes give a small response in the first column and discharge less for high weir levels. Additionally, too low weir levels restrict the water to be transferred to the second column reducing the transferred energy. The found efficiency's for wave amplitude from $a_{w}=1-3 m$ varied between $\eta=12-7.5 \%$. The lower efficiency for larger wave amplitudes is because it requires a higher weir level, which results in higher velocity amplitudes and thus higher energy losses and potential energy remained in the resonating column.

\subsubsection{Discussion}

In the derivation of the equations of motion in chapter 3, multiple assumptions have been made to simplify the equations. It is important to note that these assumptions have to be considered carefully while evaluating the results above. Some major assumptions imported to address in future work are stated in the following.

The hydrodynamic wave pressure and radiation coefficients are highly simplified. The wave pressure was computed for an undisturbed wave where the structure most will undoubtedly influence the pressures. Additionally, the wave pressure is assumed to be applied uniformly at the mouth. The small area does allow for this assumption. However, the pressure difference just below the mouth is not addressed. This same hold for the hydrodynamic end effects.

For the first column the hydrodynamic coefficients are approximated for the graphs given in [3]. These coefficients are obtained from a two dimensional model per meter width of the crest, in the simulations, this is scaled to a 10-meter wide structure, which will influence the coefficients. Additionally, the large wavelengths required the added lengths to be obtained via extrapolation, while graphs showed little linearity. The eventual found added lengths were still small compared to the total size of the structure.

In the second column exit, the hydrodynamic effects are partially neglected. The damping was assumed as pressure losses at the exit, and the added length is neglected by using the equivalent length term $L_{e q, 2}$. The pressure losses were found to influence the response of the columns significantly. These are important to obtain more precisely for future calculations. The added lengths are expected to have a smaller effect where the inertia of the second column was already very high. The second point for discussion is that; for both columns, the free surface modelled as a rigid moving piston. However, in the first column, the weir discharge redirection the vertical flow to horizontal flow and will induce an elevation variation of the free surface above the weir. In the second column, the free-surface area is much larger compared to the exit pipe, the velocity of the particles will, therefore, be directed to the exit and not vertical as assumed, causing local free-surface elevations inside the column. Also, the impact of the discharge water will cause local elevations.

Lastly, the turbine has a considerable weight in the analysis of the power take-off. However the modelling done is highly unrealistic due to the use of a stationary hydraulic turbine regression relates in a dynamic flow, the variable power take-off for constant rotational speeds and the overestimation of power related to the power available in the flow. Despite the modelling limitations, the results showed a definite limit of the extractable power. 


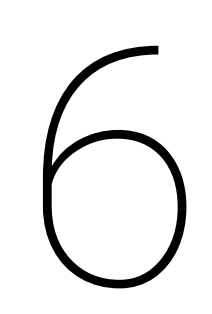

\section{Comparison scale model}

In this chapter, geometry equivalent to the model of the scale test is simulated and compared to the results of the previously executed experiments. First, the circular geometry is expressed in the same geometry as the proposed system. Subsequently, the model is simulated for the same exciting waves and depth as in the experiments and compare to the experimental results.

\subsection{Scale model}

For scale tests, a wave basin a $1 / 20^{\text {th }}$ model of the original Neptune wave energy converter design was build by David Hagan from carbon fibre. The doughnut shape design had an outer diameter of 1.98 and a height of 1.2 meters, all dimensions are presented in figure 6.1. To scale the air contribution of the air pressure, an additional air chamber is used, connected to the submerged air chamber by a semi-rigid air duct. The dimensions of the additional air chamber are $2 \times 2 \mathrm{~m}$, to which the valves to regulate the internal pressure and weir levels are connected. Lastly, the model was equipped with a removable exit duct to transport the whole device. Additionally, different duct diameters could be used during the testing. This model was tested on two occasions, in 2008 in the wave tank of the University of Strathclyde, Glasgow, and in 2013 in the wave tank of the Plymouth University.

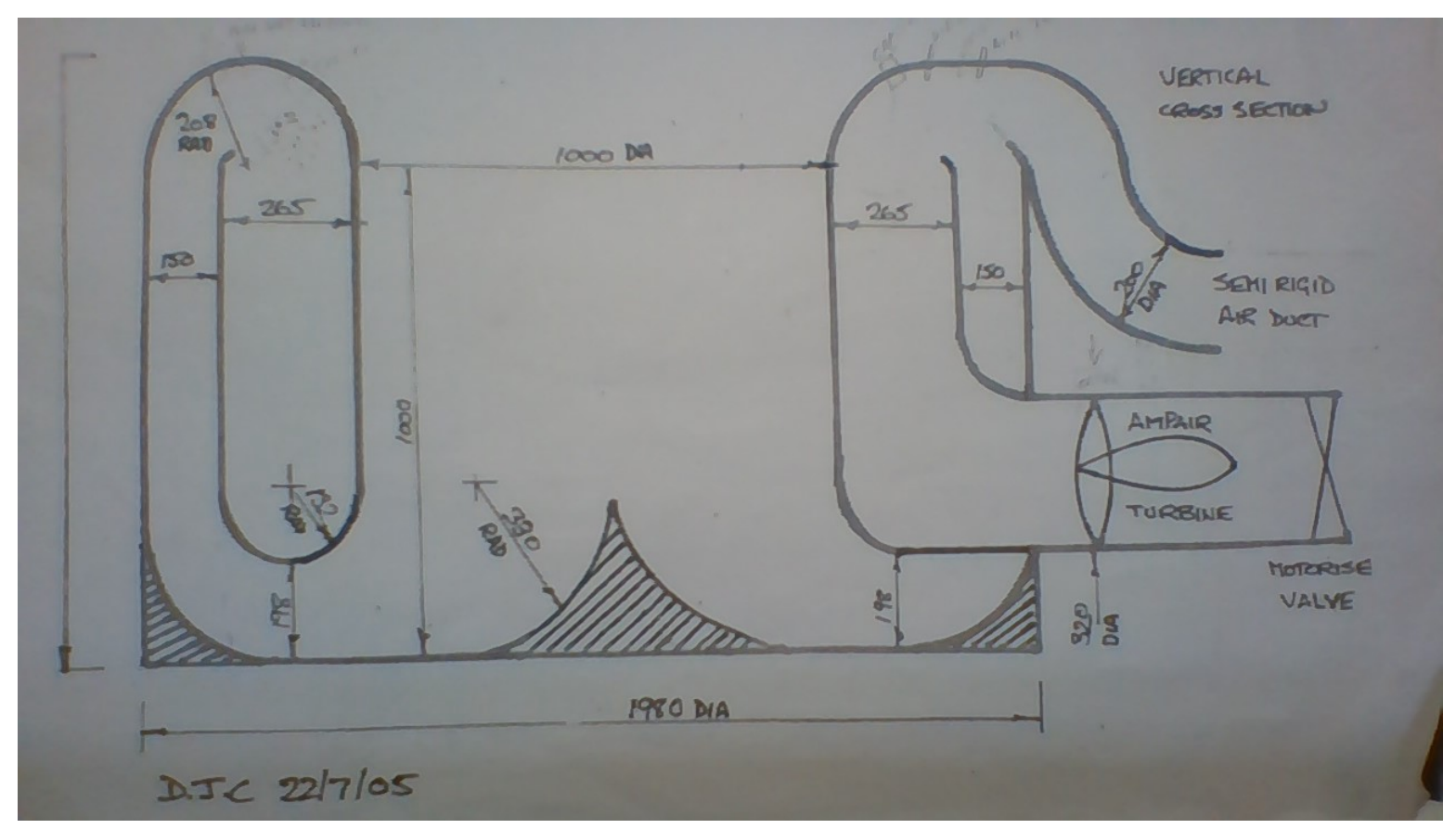

Figure 6.1: Neptune building drawing $1 / 20^{\text {th }}$ scale model 
Strathclyde University and Neptune Energy LTD supported the test in Glasgow. The goal of this test was to prove the concept and obtain an approximation of the response to a range of monochromatic wave conditions. In Plymouth the tests, commissioned by Land and Water Group, were repeated for Neptune Energy LTD and Land and Water Group to compare the compare with the previously obtained data, addressing an irregular wave field briefly.

\subsubsection{Glasgow tests}

The test in Glasgow was carried out over the time of 5 days, from January $17^{\text {th }}-18^{\text {th }}$ and $21^{\text {st }}-23^{\text {rd }} 2008$ in the Kelvin Hydrodynamics Laboratory of the Naval Architecture, Ocean and Marine Engineering department of the University of Strathclyde, Glasgow. The overall dimensions of the wave tank are $76 \times 4.6 \times 2.5 \mathrm{~m}$, and its wave maker consists fo a variable-water-depth computer controlled four-flab absorbing wave maker, generating up to $0.5 \mathrm{~m}$ high regular and irregular waves [42]. The tests have been commissioned by Neptune Energy LTD to be carried out by the University of Strathclyde. During testing the response to regular waves with periods varying from 1.8 to 2.4 seconds were established at different water depths. The weir level was varied by $z_{w}=3.5 \mathrm{~cm}, 5 \mathrm{~cm}, 6.5 \mathrm{~cm}$ and $0.75 \mathrm{~cm}$. Additionally, two different exit ducts have been used with diameter $D=19.5 \mathrm{~cm}$ and $D=24.5 \mathrm{~cm}$. During the testing, the following parameters are measured:

- Wave height before and after the device by wave probes

- Pressure in the submerged air chamber by a pressure sensor inside the air chamber

- Flow through the exit duct by a propeller.

Further, an underwater camera was used to film the inside of the air chamber and the propeller in the exit duct. The data is recorded for a frequency of $60 \mathrm{~Hz}$; the specific instrument specifications could not be traced back. From the experiments, the filtered time domain pressures and flow rates are available for the analysis, together with a summary of the results, including the settings of the test, presented in appendix D.

\subsubsection{Plymouth tests}

The tests in the have been performed 5 years after the first test in Glasgow. The test has been performed by 'Coast Laboratory' of 'Plymouth University' commissioned by 'Land and Water Group' together with Neptune Energy LTD. The coastal wave basin of the COAST Laboratory is $35 \mathrm{~m}$ long and $15.5 \mathrm{~m}$ wide with operational depths up to $3 \mathrm{~m}$. Equipped with 24 flap multidirectional wave makers with a $2 \mathrm{~m}$ hinge depth, producing regular waves of up to $0.9 \mathrm{~m}$ in height. The objectives of the experiments were:

- to establish the response of the 'Neptune' wave energy converter to a range of monochromatic wave conditions in terms of power extracted from the wave field.

- briefly examines the response in an irregular wave field.

- to enable comparison with the previously obtained data in the Glasgow test.

- visualise the internal behaviour of the device.

During testing the pressures in the inner air chamber were measured by two pressure sensors (Measurement Specialities XPM10-A2-1BS-/I8M), an additional pressure sensor was added in the outlet pipe. To measure the water velocity, a mechanical impeller was used of the type 'Valeport Braystoke BFM002'. The impeller has a minimum flow velocity of $0.6 \frac{\mathrm{m}}{\mathrm{s}}$ with a large uncertainty below $0.15 \frac{\mathrm{m}}{\mathrm{s}}$. Together with the pressure sensors and the impeller, four underwater video cameras were installed. Three of these cameras were located within the air chamber, capturing imaged for the internal free surface levels. The fourth camera was positioned near the exit of the outlet points, filming the behaviour of the impeller. A $10 \mathrm{~Hz}$ low-pass filter has been used to process the pressure signals. The behaviour was tested for different wave heights $\left(H_{w}=0.05,0.15 \mathrm{and} 0.25 \mathrm{~m}\right)$, weir levels $\left(z_{w}=35,50\right.$ and $\left.65 \mathrm{~mm}\right)$ and water depths $\left(d_{w}=1.85,2.35\right.$ and $\left.2.85 \mathrm{~m}\right)$. From the experiment, only the summarized results are available and can be found in the test report, [43]. Additionally, the diameter of the exit duct was not specified. 


\subsubsection{Simulated model}

The model analysed in the previous chapters was modified to a square structure instead of the circular doughnut design proposed by Denis Carey. From the circular model, the areas and lengths of the columns are calculated and used in the analysis. The width mouth for the radiation force damping is assumed to equal to the diameter of the mouth of the first column. The dimensions representing the $1 / 20^{\text {th }}$ model in the numerical analysis are presented in table 6.1.

Table 6.1: Dimensions initial frequency analysis for a structure width of 10 meter, corresponding to figure 3.2

\begin{tabular}{|c|c|c|}
\hline Variable & unit & value \\
\hline$L_{c 1}$ & $m$ & 1 \\
\hline$L_{1}$ & $m$ & 1.4 \\
\hline$L_{c 2}$ & $m$ & 0.5 \\
\hline$L_{2}$ & $m$ & 3 \\
\hline$A_{1}$ & $m^{2}$ & 7850. \\
\hline$A_{c 1}$ & $m^{2}$ & 0.84 \\
\hline$A_{c 2}$ & $m^{2}$ & 1.1 \\
\hline$D_{2}$ & $m$ & $0.195 \& 0.245^{2}$ \\
\hline$V_{0}$ & $m^{3}$ & 5.5 \\
\hline
\end{tabular}

The two experiments combined a large number of test runs have been performed. With the measured data of the time domain response of the Plymouth test not available and the dimensions of the exit pipe unknown, these will not be considered in this comparison. In the Glasgow test, the diameter of the exit pipe is given for each test run, the length of the pipes is estimated from the available pictures and movies of the testing $L_{2} \approx 3 \mathrm{~m}$. Additionally, while analysing the data of the wave amplitudes, it was found that the measured wave height differed from settings of the wave maker. Therefore in the numerical analysis, the measured wave height is used for the comparison. As can be seen from the experiment parameters in appendix D, the majority of the test was done for an inlet depth of the mouth of $50 \mathrm{~cm}$ and a wave height of $15 \mathrm{~mm}$. For these settings, the wave period was varied between $1.8-2.4 \mathrm{~s}$ for weir levels of $z_{w}=35,50$, and $75 \mathrm{~mm}$. In these cases, the minimum, maximum and mean measured velocities are obtained from the data record together with the actual measure wave height. These data will then be compared to the results of the numerical model with the equivalent parameters.

\subsubsection{Results}

In this section, the results of the numerical simulated and the Glasgow experiments are presented. For the comparison, the velocities in the exit pipe are used. In figure 6.2 the measured response during the experiments for a weir level of $z_{w}=50 \mathrm{~mm}$ and the diameter of the exit pipe with the value $D=195 \mathrm{~mm}$ is presented, run 35. In the velocity graph, the oscillations with a positive mean are visible, concluding to a uni-directional, together with an air pressure difference in the air chamber around zero. For the wave response, amplitudes of $a_{w} \approx 0.9-0.12 \mathrm{~m}$ are observed. The measurements are higher than the $0.75 \mathrm{~m}$ given in the experimental setup for the wave height in this run. Lastly, a reduction of the wave amplitude was found up and downstream of the device, case by the dissipation of wave energy by the Neptune and the reflection.

In figure 6.3, 6.4 and 6.5 the numerical obtained results are presented with the experimental values. For this setup, two sequences of different wave periods are performed during the experiment, run 18-21 and run 31-39. From the figures, the similarity in shape can be seen. In the experimental results, a higher peak period is observed compared to the numerical model. As described in the previous chapter, this could be a result of the difference in modelled damping compared to the actual damping. The difference can also be a result of an underestimated length of the added length of the resonating column, an overestimation if the air pressure stiffness or a higher weir discharge. The overestimation of the air pressure stiffness is likely a result of the a to small initial air pressure or overestimation of the polytropic coefficient $\gamma=1.4$. The volume was already assumed on the upper bound of the design drawing dimensions.

Additionally, the measured velocities are higher. In figure 6.5 this difference is unlikely, where the velocity

\footnotetext{
${ }^{1}$ Assumed value: The length is not specified in the drawings or results papers

${ }^{2}$ Two different sizes are used during the experiments
} 
for low periods are positive. The decreasing line indicated an increasing response to the wave period without weir discharge, a positive minimum velocity is therefore highly improbable and is presumably the result of a mistake in the calibration, flow calculation, or measurement inaccuracy.
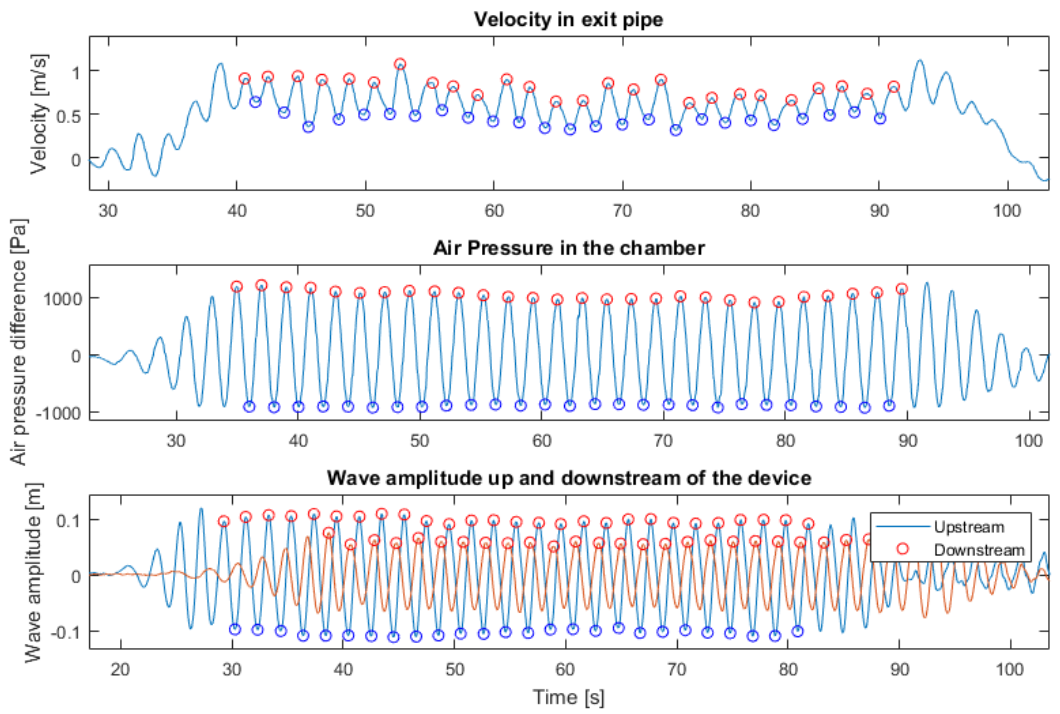

Figure 6.2: Time domain response experiments, for $z_{w}=50 \mathrm{~mm}$ and $T_{\text {measured }}=2.1 \mathrm{~s}$ and an exit pipe diameter of $\mathrm{D}=195 \mathrm{~mm}$

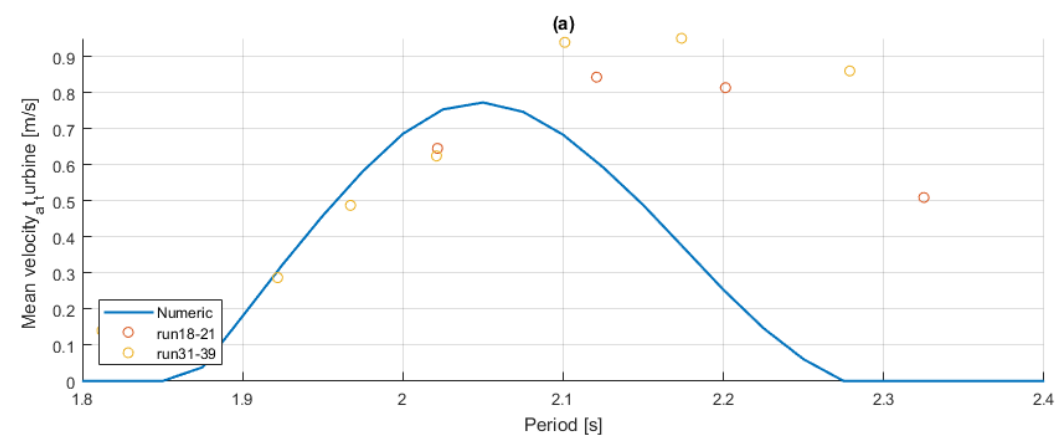

Figure 6.3: Mean velocity for different wave periods for a weir level of $z_{w}=50 \mathrm{~mm}$ and an exit pipe diameter of $D=195 \mathrm{~mm}$

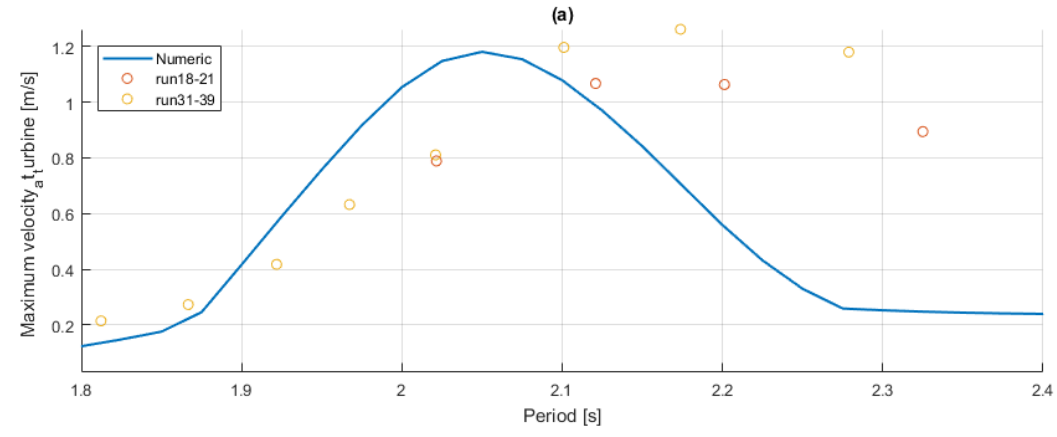

Figure 6.4: Maximum velocity for different wave periods for a weir level of $z_{w}=50 \mathrm{~mm}$ and an exit pipe diameter of $D=195 \mathrm{~mm}$ and an exit pipe diameter of $D=195 \mathrm{~mm}$

In figures 6.6, 6.7 and 6.8 the results for a weir level of $z_{w}=75 \mathrm{~mm}$ are presented. The results show the same trends as in the previously described situation with a weir level of $z_{w}=50 \mathrm{~mm}$. A major difference, however, is observed in the bandwidth of the numerical model. In the numerical model, an amplified response 


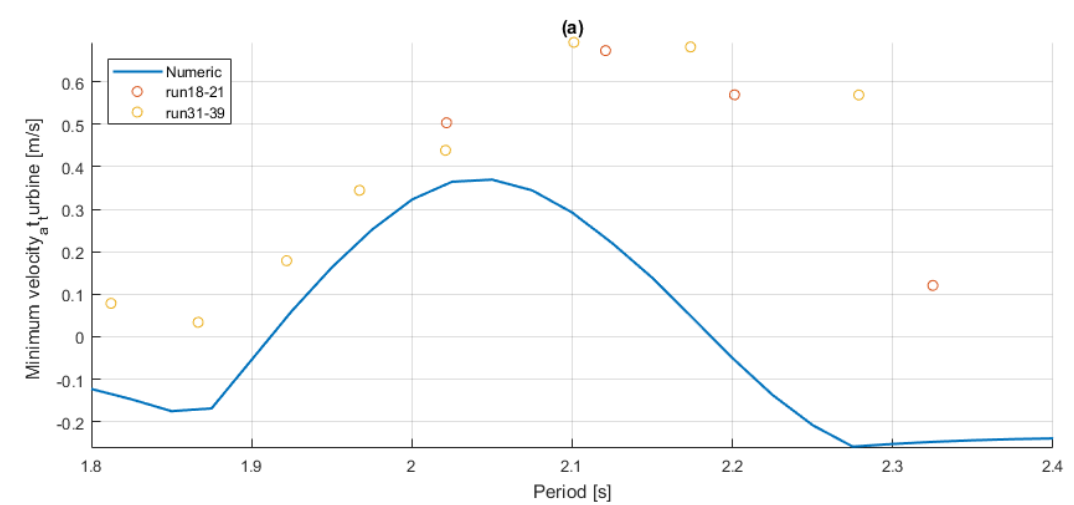

Figure 6.5: Minimum velocity for different wave periods for a weir level of $z_{w}=50 \mathrm{~mm}$ and an exit pipe diameter of $D=195 \mathrm{~mm}$

is observed for a smaller range of wave periods. This could indicate that the motion of the first column is underestimated in the numerical model. The experiment with a weir level of $z_{w}=65 \mathrm{~mm}$ only consists of three runs and will therefore not be presented. The found relation in the numerical model was in line with the previously presented results of the two other weir levels.

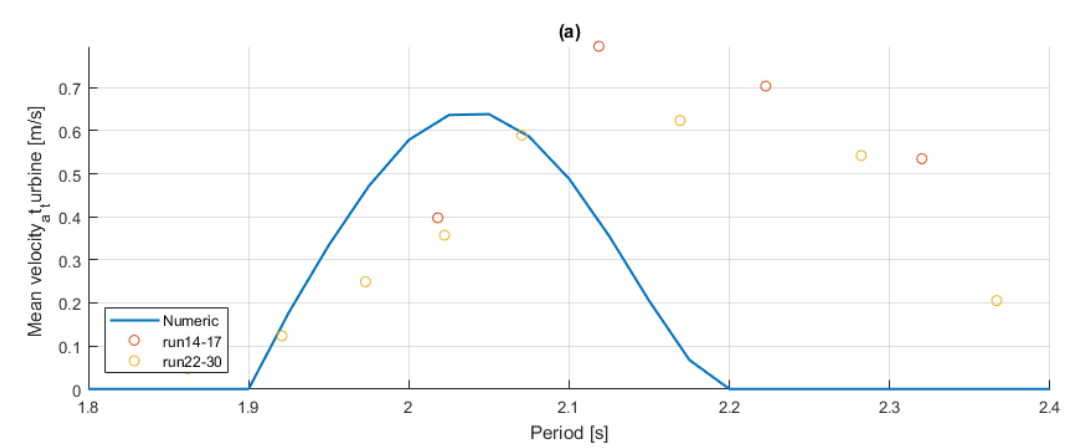

Figure 6.6: Mean velocity for different wave periods for a weir level of $z_{w}=75 \mathrm{~mm}$ and an exit pipe diameter of $D=195 \mathrm{~mm}$

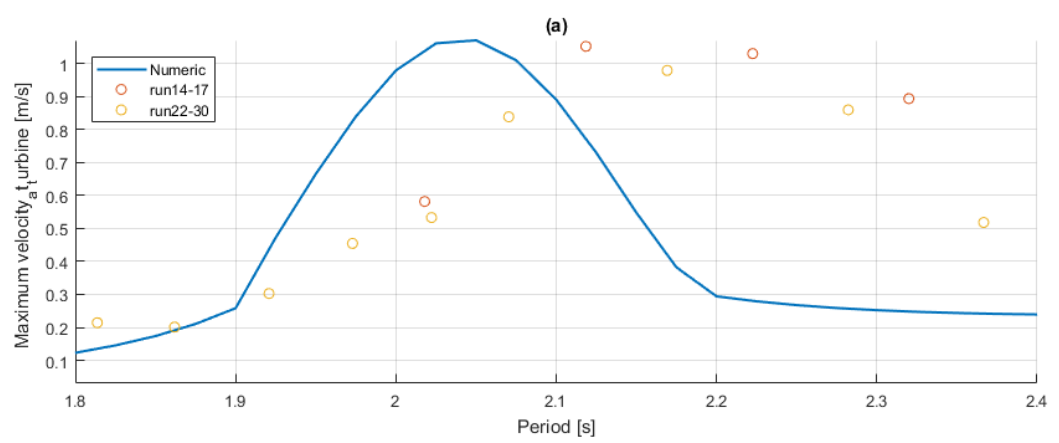

Figure 6.7: Maximum velocity for different wave periods for a weir level of $z_{w}=75 \mathrm{~mm}$ and an exit pipe diameter of $D=195 \mathrm{~mm}$ 
(a)

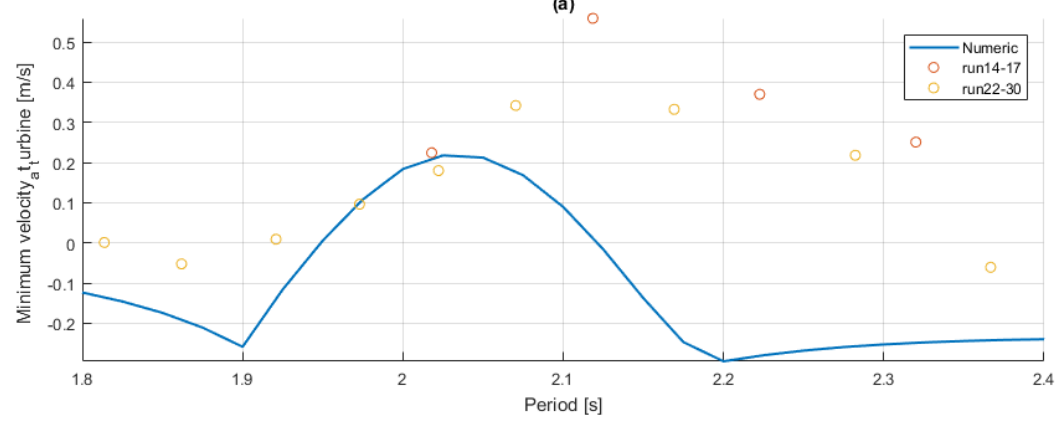

Figure 6.8: Minimum velocity for different wave periods for a weir level of $z_{w}=75 \mathrm{~mm}$ and an exit pipe diameter of $D=195 \mathrm{~mm}$ 
The second experiment sequence was done for an exit pipe diameter of $D=245 \mathrm{~mm}$; for this setup, 3 different weir levels were tested that all obtained valid results for the analysis. The comparison between the measured mean velocity and the numerically obtained value are presented in figures $6.9,6.10$ and 6.11 . In the comparison, it can be seen that the numerical results are closer to the measured response, in terms of the magnitude, peak period and bandwidth of response. Further, compared to the $D=195 \mathrm{~mm}$ setup, the measured velocities are lower while the discharge is larger, caused by the increased area. For the increased discharge higher peak periods would be expected. However, the measured peak periods decreased slightly. Additionally, the slopes of graphs for higher periods is found less steep. The same effect was obtained in the numerical model.

In the modelling, the only different parameter is the inertial term of the second column, which is smaller for the $D=245 \mathrm{~mm}$ as a result of $\frac{A_{c} 2}{A_{2}}$. This is therefore assumed to be related to the slope of the graphs and the response of the first column. Concluded a lower inertial term in the second increases the response amplitudes in the first column, resulting in a higher discharge, increasing the response for a broader range of periods.

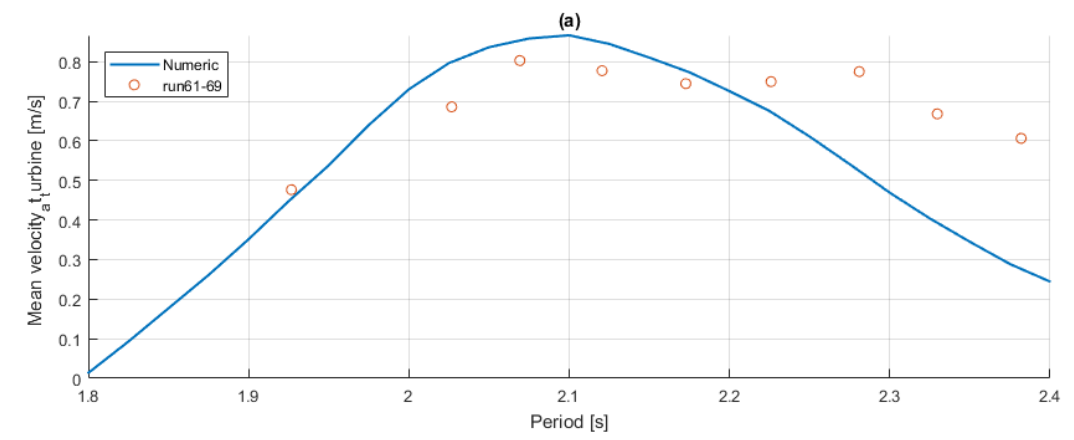

Figure 6.9: Mean velocity for different wave periods for a weir level of $z_{w}=35 \mathrm{~mm}$ and an exit pipe diameter of $D=245 \mathrm{~mm}$

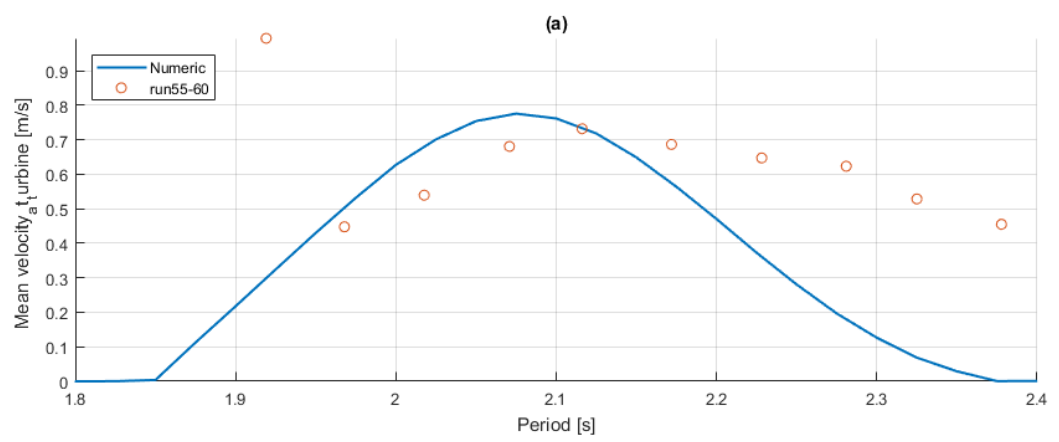

Figure 6.10: Mean velocity for different wave periods for a weir level of $z_{w}=50 \mathrm{~mm}$ and an exit pipe diameter of $D=245 \mathrm{~mm}$

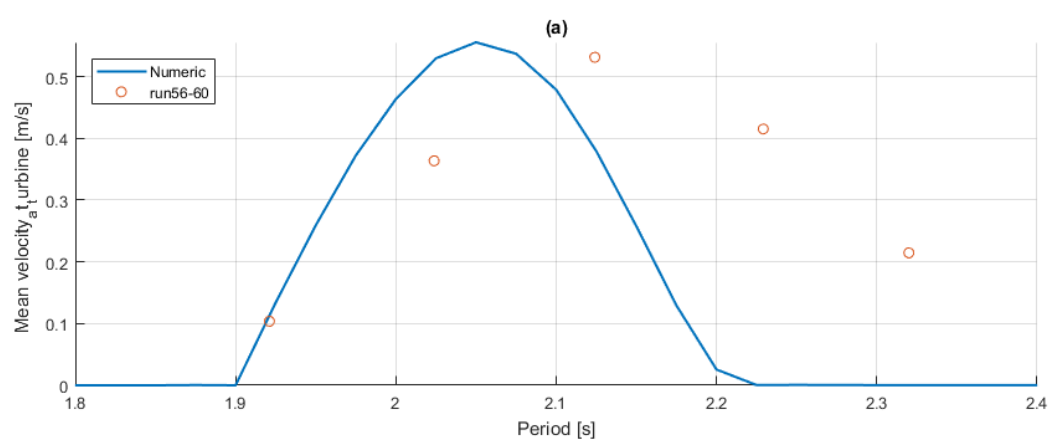

Figure 6.11: Mean velocity for different wave periods for a weir level of $z_{w}=75 \mathrm{~mm}$ and an exit pipe diameter of $\mathrm{D}=245 \mathrm{~mm}$ 


\subsection{Conclusion and discussion}

\subsubsection{Conclusion}

From the measured of the following conclusions can be drawn with respect to the numerical model:

- The response of the numerical model was found to obtain results in the same order of magnitude with respect to the experimental results

- The peak period of the measured data was higher than the numerical results

- At higher periods, the numerical response is underestimated compared to the experimental results.

Further, some additional observations are found with respect to the response of the system.

- The inertia term of the second column affects the dynamics first column, with a high inertial term resulting in:

- Reduced amplitudes of the first column response

- lower discharge

- steeper slope of the graphs, reducing the bandwidth of the system

\subsubsection{Discussion}

From the conclusions above a good relation between the numerical model and the scale model was found. However, the following remarks have to be made on the comparison.

- The numerical model is made for a square structure for which the width could be varied. The scale model was a circular shaped structure.

- The three-dimensional effect of the circular shape is not taken into account.

- The modified dimensions are assumed from the drawing. The dimensions of the scale model might be different from the actual dimensions, where the dimensions of the manually build scale model could not be verified with the drawing in figure 6.1. Additionally, it is unclear if these were the final drawings for the build. The dimensions are tuned to obtain a system with the design frequency of 2 seconds for an assumed atmospheric air pressure.

- The length of the exit pipe is unknown and is estimated, while this term was found to have a big influence on the overall behaviour of the system.

- The air pressure are not compared because:

- The atmospheric pressure during the test, which affects the air chamber pressure, is not known for the tests are estimated as the average atmospheric pressure at sea level.

- the measure pressures were lower than the numerically obtained pressure, With the modelled air volume and inertial term of the first column estimated at the upper limit with respect to the dimensions of the model.

- The measured air pressures were not stable during separate runs, indicating an error in either the measurements or the filtering of the signal.

- The additional air chamber appeared to be not completely rigid, resulting in variations of the air volume

- The dimensions of the scale model might be different from the actual dimensions, where dimensions of the manually build scale model have not been verified with the drawing in figure 6.1 and it is unclear whether these were the final drawings. The dimensions are tuned to obtain a system with the design frequency of 2 seconds for the assumed atmospheric air pressure.

- The wave amplitudes were found variable between different runs. The measured wave height was also higher than the settings of the wave maker. Making the correctness of the data more arguable. 


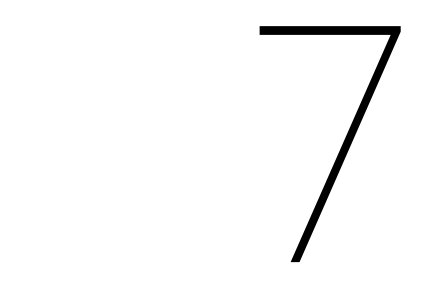

Feasability

In the previous chapters, the working principles of the Neptune have been analysed together with the performance. In this chapter, this performance is compared to other wave energy converters. Furthermore, matters associated with the construction and installation are addressed. Finally, some design alternatives are presented to increase the scale of the structure and optimize its performance. It is important to note that the values used in this chapter are obtained per meter width of the structure.

\subsection{Power available at location}

In chapter 5, the efficiency of the power take-off are presented. From this data, the efficiency for different wave heights and periods can be obtained. In earlier work on oscillating water columns, a close relation was found between the results of regular waves and irregular waves with corresponding peak periods[44, 45]. In [44] capture width of 1-13\% were found for irregular waves against $2-10 \%$ for an irregular wave spectrum. In [45], a slight increase in the normalized response amplitude of the inner free-surface was found for an irregular wave spectrum compared to the regular waves. Therefore it is assumed that efficiencies found in chapter 5 are in the same order of magnitude as the irregular waves. It is important to note that the average power in the irregular wave spectrum is only half the power available in a single regular wave for the same wave height and period, as was seen in equations (2.6) and (2.9). The found efficiencies can now be related to the measures wave data of the proposed site in chapter 4 . For each sea state, the efficiency can be found in the results. It is assumed that the weir levels will be changes to obtain the optimal efficiency for each wave condition. The efficiency for various significant wave heights and spectral peak periods, is presented in figure 7.1b. In figure 7.1a, the average annual joint frequency distribution of the wave conditions. In the efficiency diagram, the efficiencies obtained directly from the results are presented with an underscore. The other values are extrapolated from the trends observed in the results. For each wave conditions, the available mean power per meter in the wave spectrum is calculated by equation (2.9). From the available power and the efficiency of the extracted power in each sea state can be determined.

The available and extracted powers are presented in figure 7.2a and 7.3a. Additionally, from the power results, the annual available and extracted energy can be determined. Multiplying both powers by the occurrence and duration of the sea states that the total annual available and extracted energy generation can be obtained. These values are given in figure 7.2b and 7.3b. From the summation of all values, the efficiency can be determined. The total available energy is $600299 \mathrm{kWh} / \mathrm{m} /$ year while $15740 \mathrm{kWh} / \mathrm{m} /$ year is extracted; this results in an annual efficiency of $2.6 \%$.

The corresponding annual available power of $P_{a v}=69 \mathrm{~kW}$, this corresponds with the values found in figure 2.1 at this location. The extracted annual power is $P_{e x}=2 \mathrm{~kW}$. In the scatter diagram of the wave height and amplitudes the majority of the sea states is found around a wave period of 8 seconds, and a wave height between 1.5 and 2 meters, however available power has its peak around 11 second and a wave height of 4.5 meters. The efficiency of the device around these sea states is low. By merely shifting the efficiency results to higher periods, a higher annual power generation was found. The optimal shift was found to be 2 seconds. This would correspond to a natural design frequency of 10.5 seconds, with a high optimal energy yield around 11-11.5 seconds. The annual power extraction for this case is increased to $17000 \mathrm{kWh}$, increasing the efficiency to $2.8 \%$. It is important to note that these results are excluding the effect of the wave direction. 


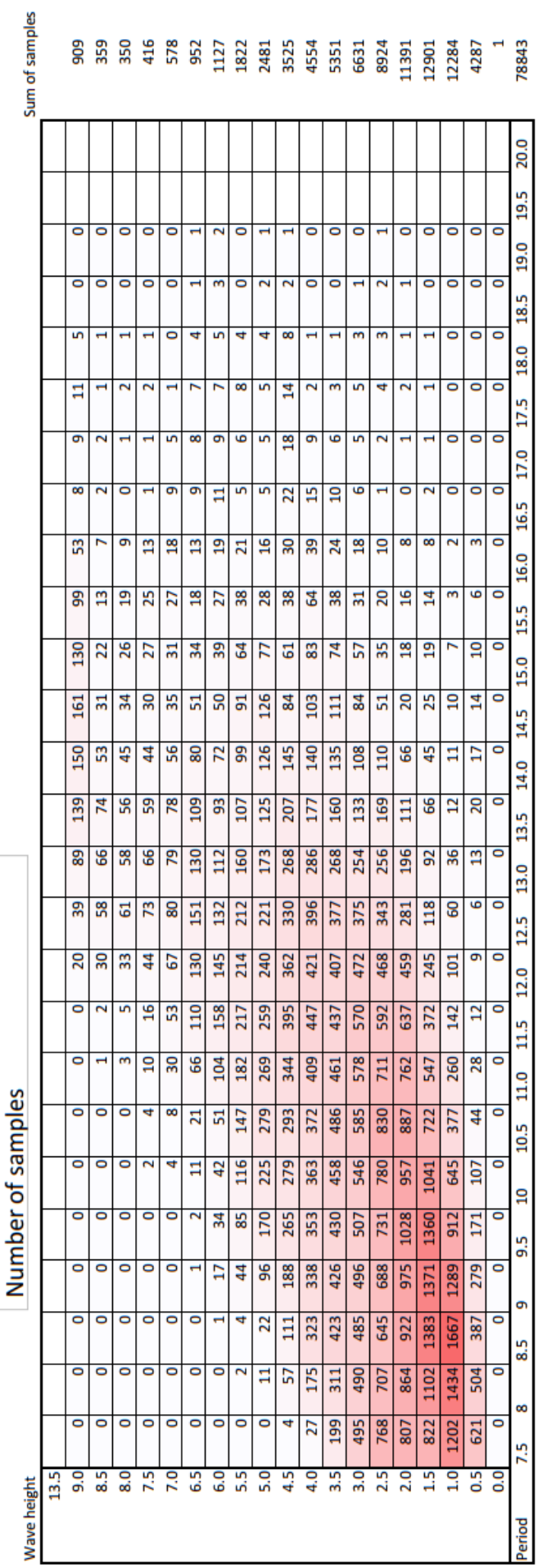

(a) Wave scatter diagram

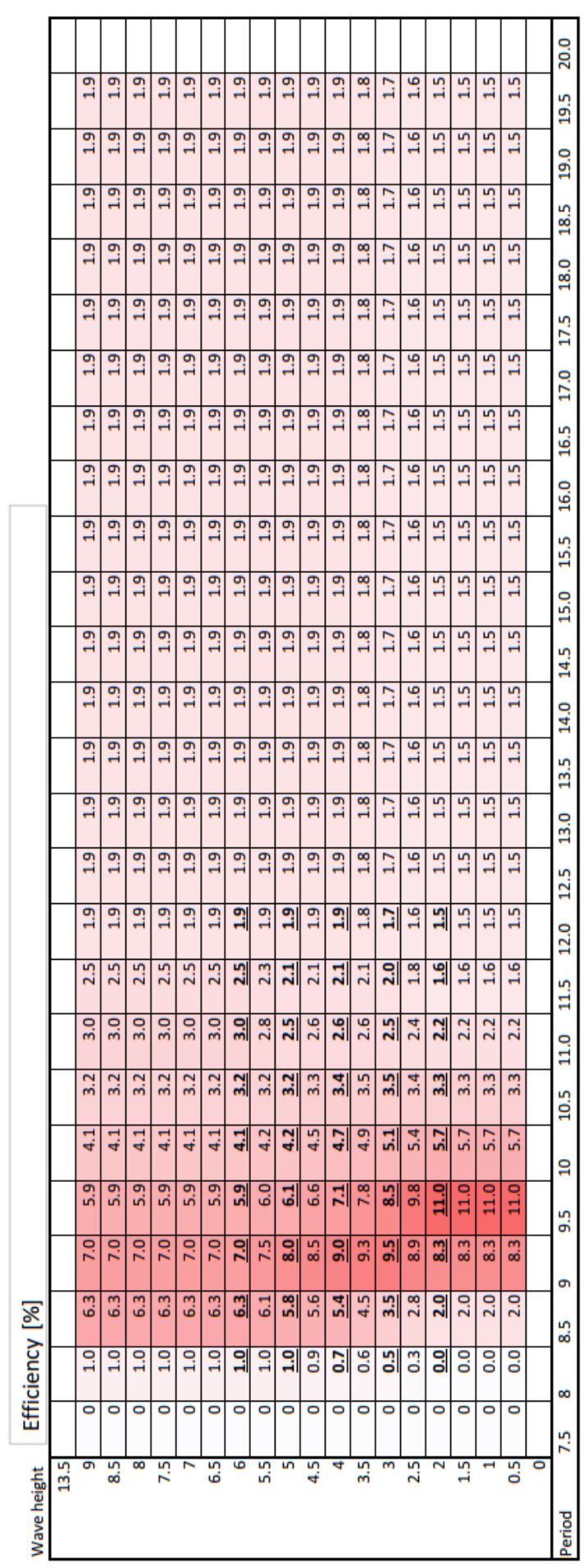

(b) Efficiency power take-off sea-states 


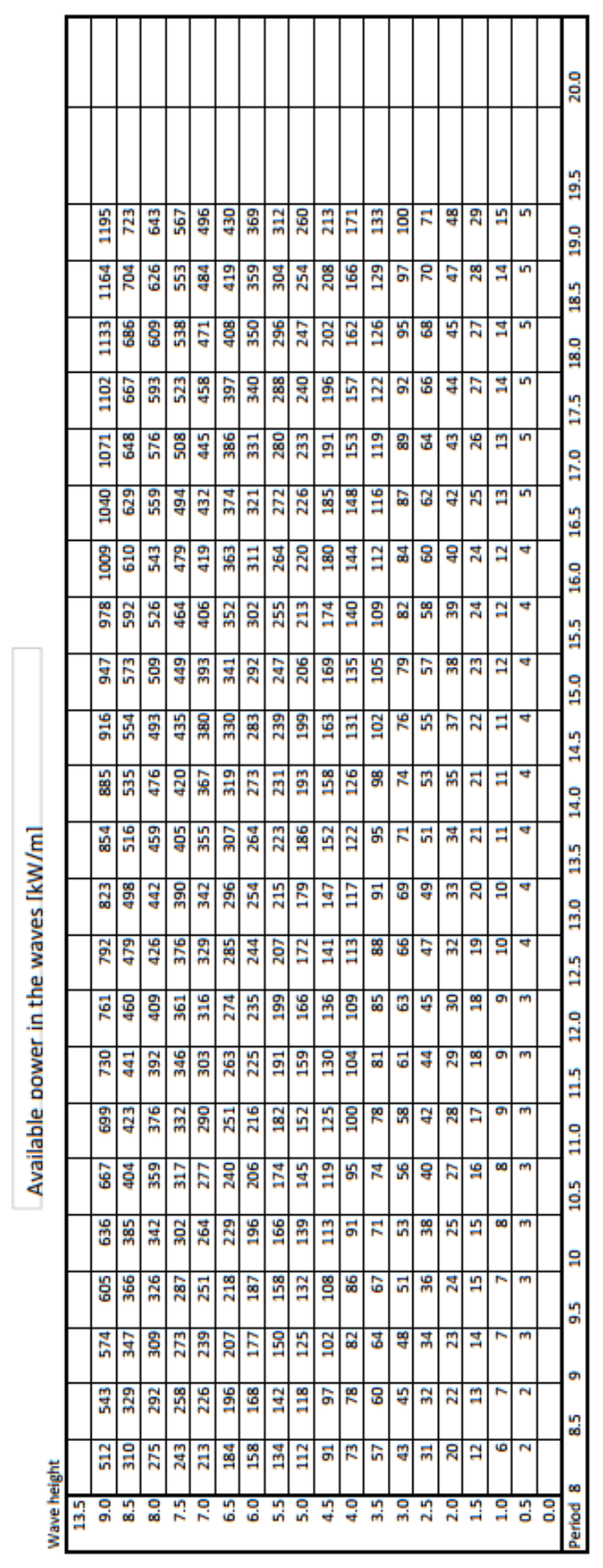

(a) Total wave power per sea-state

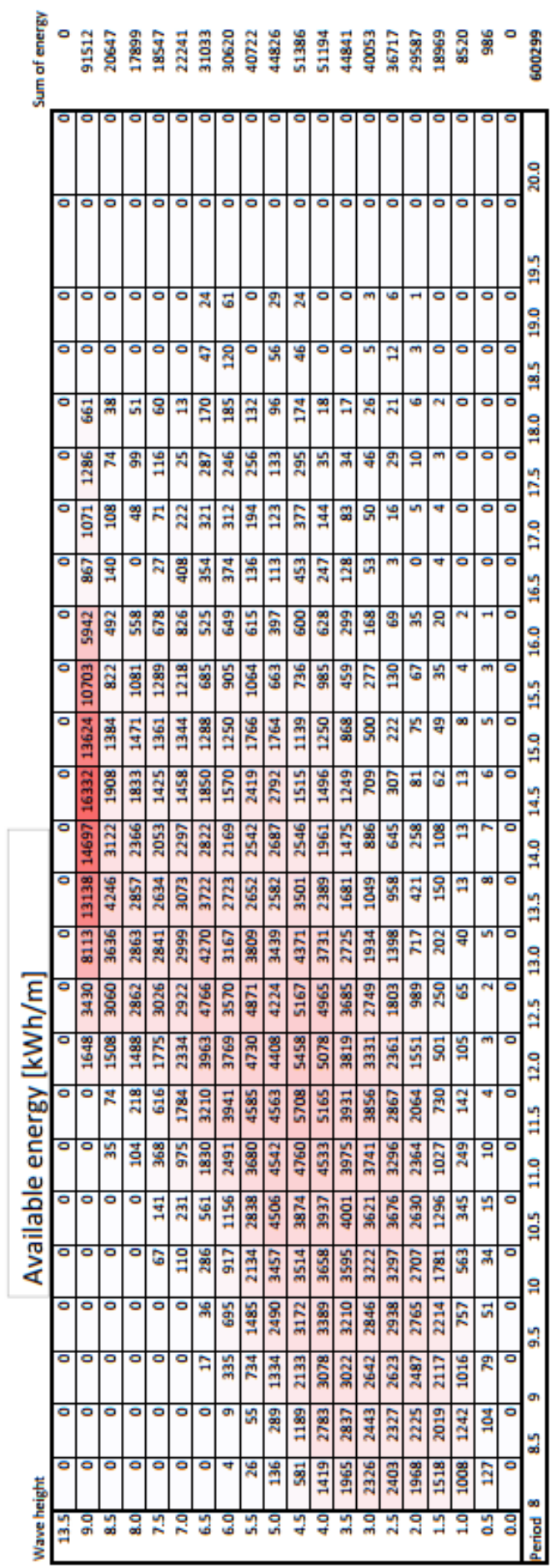

(b) Average availble annual energy 


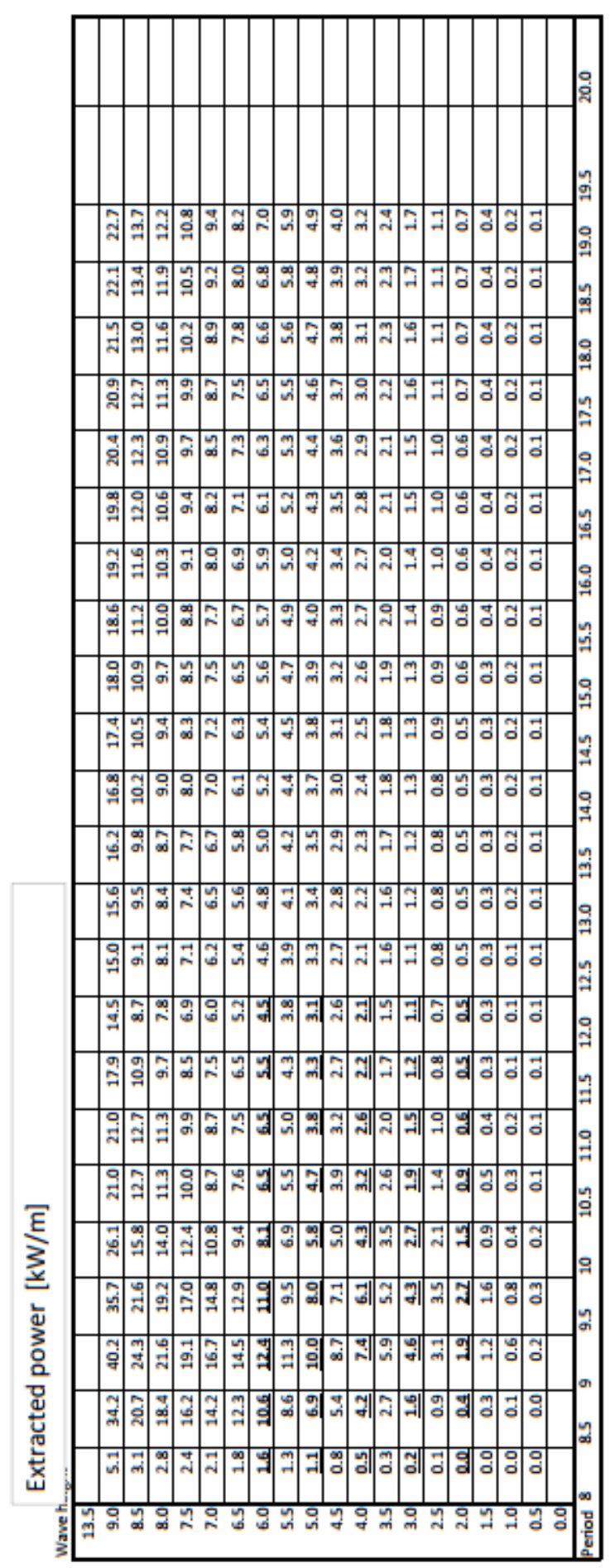

(a) Extracted power from specific sea-states

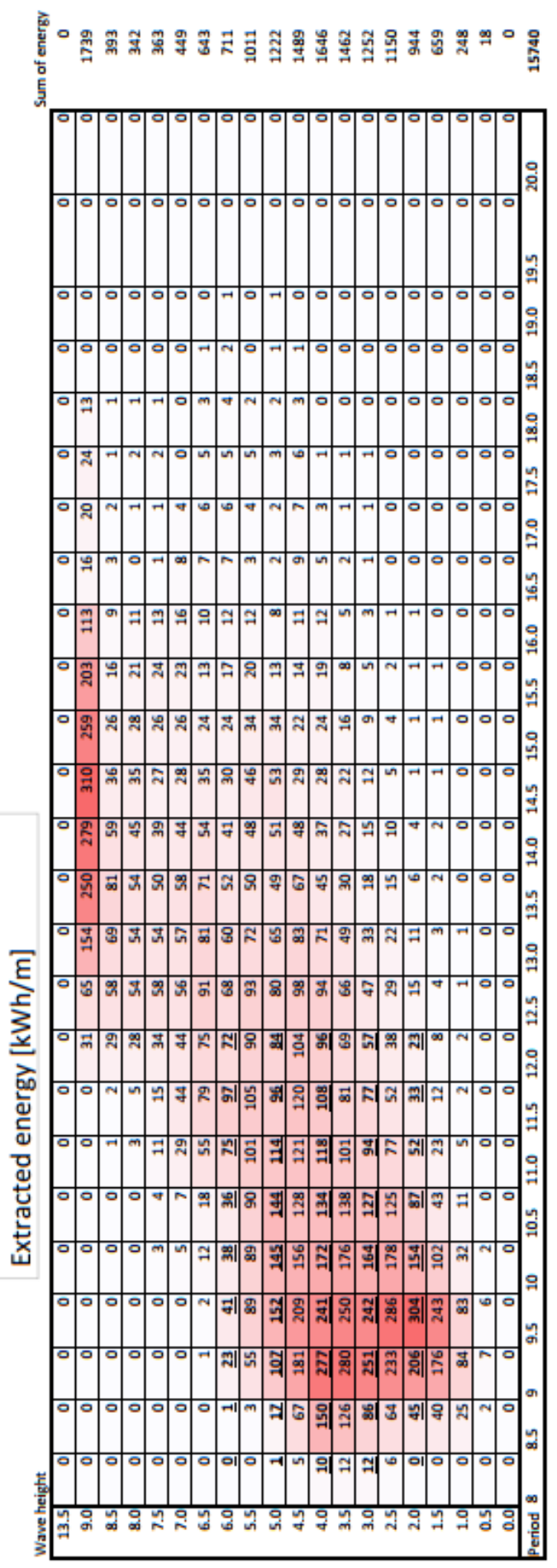

(b) Average annual extracted energy 


\subsection{Main structure}

This section shortly addresses the global shape, dimensions and required weight of the structure housing the water column. The exit pipe of the second column is addressed separately in the next section. The design of the structure can be rather simple, a rectangular shaped structure with two vertical walls inside, as presented in figure 7.4. One of the walls is suspended from the top of the structure, forming the inlet duct of the first column. The second wall, with its top the weir level separating the two water columns. Lastly, a relatively small diameter exit duct connecting the second column to the turbine and the surrounding water. The installed structure is completely submerged and contains a large internal air pocket; this large air chamber causes buoyancy of the full structure. The dependence on the volume of the air chamber disables to the possibility to reduces its size without reducing the performance. To avoid the structure from emerging to the surface after installation, several options are available:

- Gravity based: Increasing the weight of the structure significantly

- Foundation: The use of tension piles to secure the structure to the ocean floor

- Mooring: Design of a submerged floating structure and keep it in position using mooring lines

The two latter options are more complex to the gravity-based method. Therefore for the first estimation on the requirements of the structure, the gravity-based structure is assumed. To obtain the first estimation of the required weight, the buoyancy of the air chamber is calculated by:

$$
F_{\text {buoy }_{a} i r}=V_{0} \rho_{\text {water }} g=200 \mathrm{~m}^{3} \times 1025 \frac{\mathrm{kg}}{\mathrm{m}^{3}} 9.81 \frac{\mathrm{m}}{\mathrm{s}^{2}} \approx 2 \frac{M N}{\mathrm{~m}}
$$

This is much weight to be compensated by the used material. Therefore concrete is proposed as a building material for this structure. Concrete is known to be capable of surviving in sea conditions where it is a common building material for offshore structures, such as breakwaters, caissons and gravity based offshore platforms. The knowledge in design in this area can be of assistance in further design and production. Now, to balance the buoyancy force a large concrete volume with density $\rho_{\text {concrete }} \approx 2400 \frac{\mathrm{kg}}{\mathrm{m}^{3}}$ is required. The displaced volume is by the concrete must also be taken into account; the total volume of concrete required for is $V_{\text {concrete }}>150 \mathrm{~m}^{3}$ to balance the $200 \mathrm{~m}^{3}$ of air. The total wall area of the structure in figure 7.4 equals $A_{\text {wall }, t}=100 \frac{\mathrm{m}^{3}}{\mathrm{~m}}$, assuming a top and bottom slap of $A_{t, b}=20 \mathrm{~m}^{3} / \mathrm{m}$, two outer vertical walls of $A_{\text {wall }, \text { out }}=20 \frac{\mathrm{m}^{3}}{\mathrm{~m}}$ and two inner walls with height $h_{w}=10 \mathrm{~m} A_{\text {wall }, \text { in }}=10 \frac{\mathrm{m}^{3}}{\mathrm{~m}}$. This results in a required wall thickness of $t_{\text {wall }}=1.5 \mathrm{~m}$. This appears to be a massive thickness for the purpose of this design. However, in caisson design, functional requirements may lead to wall thicknesses up to 3 meter [46]. It is proving the feasibility of the construction of such a structure.

In the above, it is assumed that the full weight is introduced by concrete. This could be partially resolved in rock filling material; this could be integrated within the design of the rectangular sections of with at the support section of the turbine and exit pipe, which are not present along the full width of the device. This section is to give an indication of the design the proposed structure, the structural integrity against the external wave and internal air pressure forces is not addressed in this research.

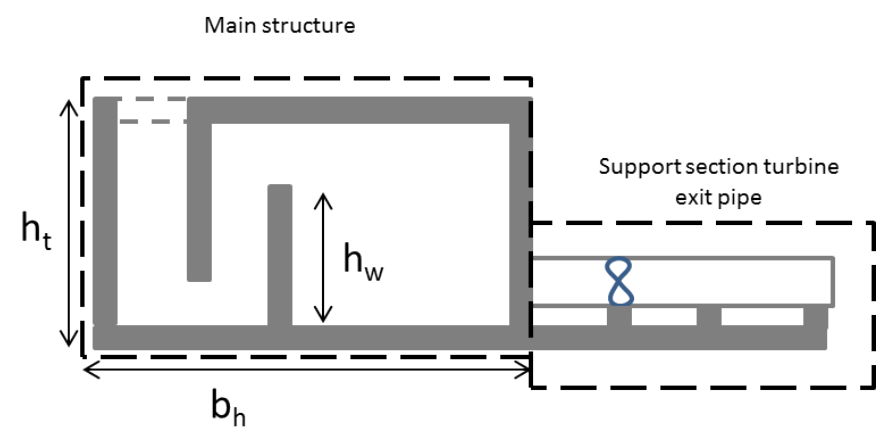

Figure 7.4: Simplified shape of the column structure 


\subsection{Turbine}

In the section above a simplified design for the structure housing, the oscillating water columns are given. However, additional to this structure that captures the wave energy a turbine is required to generate this power. Identical to the section above the design of the turbine interface is not addressed in detail in this section, but general thoughts and practical elements on the design will be addressed. One significant advantage of the analysed design is the simple, durable structure with a low number of movable parts, namely the turbine. In practice implementing a hydro turbine is a bit more complicated, where the hydraulic turbine is not known for there low maintenance requirements [47]. This, in combination with limited offshore accessibility under water, requires suitable solutions.

One option is to increase the number of turbines used in the structure. This might also be required if the total width of the structure is increased to capture wave energy over a larger width. The multiple turbines can work in parallel op optimize the energy generated when much water is discharged into the second column. Simultaneously the turbines can be turned on and off separately to optimize the performance of the turbine for the current sea stated. Lastly, this approach could enable a single turbine to undergo maintenance or repairs while avoiding downtime of the full system. It has to be considered that for this system to work, closing valves are required in front of the turbine, while valves increase the pressure losses and thus reduce the available power that can be generated.

The second possibility is to enable the turbines to be easily replaced from above water. This way, the maintenance and repairs don't have to be executed underwater, reducing the complexity and possibly the costs of the operation. This option does require a connection to the exit pipe that is easily attached and detached without introduction too much pressure losses. Additionally, the connection to the power grid has to be flexible to either rise to the surface together with the turbine or to be coupled and decoupled underwater.

Both options can be used independently of each other but might increase the efficiency of the project when used both. Apart from these possibilities, the turbine has to be connected to the exit pipe of the second column. While the fabrication, transportation and installation of the concrete structure, described in the next section, is preferred to be a stand-alone project the connection of the turbine and its auxiliary items are to be installed afterwards. The concrete structure is to be designed with an integrated foundation for the turbine and its auxiliary items.

\subsection{Fabrication, Transportation and Installation}

In this section, a proposal is made regarding the Fabrication, transportation and installation of the proposes rectangular concrete gravity-based structure. Given the dimensions of the structure, it is most appropriate to prefabricate the structure before placing it in the final position. The construction site has a large influence of the logistics of the full project, including the fabrication, transportation and installation. Three potential facility types are[48]:

- Dry docks

- Floating pontoons

- Onshore in quay areas

The possible transportation method for all three facilities are [49]:

- Barge transport

- Individual transport by heave lift vessels

- Self floating structures

In the previous section, a relatively large weight was found per meter with of the structure, together with a low annual efficiency found for the device it will require a large width. The transport on a barge or by heavy lift vessel is therefore assumed to be not feasible. The remaining option is the self floating structure, the massive air chamber inside the structure and the shape of the structure does facilitate this type of transportation. The self-buoyant type structures can be towed by standard tugs, which have high availabilities and are relatively cheap. The most optimal fabrication site for this type of transport is a dry dock. After construction, the dock can be flooded, and by increasing internal air volume the structure can be made buoyant and tugged out of 
the dry dock. This method averts the need for heavy lift vessels. The long rectangular structure can be seen as a close resemblance to the immersed tunnel sections, which are also transported via buoyant transport.

Following the transportation of the structure, it has to be installed. Prior to the installation, the seabed must be prepared, which is a severe disadvantage for gravity based structures. The main objectives of the seabed preparation are to obtain sufficient bearing capacity and level the seabed, the requirements of the seabed preparation depend on the bathymetry, seabed features, subsurface conditions, sea and water conditions and the amount of unsuitable debris on the seabed. The standard procedure of seabed preparation is based on two phases. The first phase is the dredging of the first layer of material on the seabed until an undisturbed soil if found. This layer usually consists of clay or loose sand. Standard layer thickness removed by dredging range from 0.5 to more than 10 meters. Dredging is generally caries out by Cutter Suction Dredgers or Trailing Suction Hopper Dredgers. After sufficient dredging, gravel or rig-rap rock bed is dropped by fall pipe vessels aided by a steel frame on the bottom acting as a levelling guide.

After finalizing the seabed preparation, the structure can be installed at its final positions. The installation of a buoyant structure is a complex operation. Despite not being dependent on the big equipment, it is technically more difficult. To sink the structure to the bottom part of the chamber is filled with water, increasing the weight in the water. Several options are available to guide the lowering of the structure. The position can be controlled by means of towing vessels with the help of anchors, pontoons or by using a jack-up vessel above the structure. The latter two methods are conventional in the installation of an immersed tunnel section for its high accuracy. However, assuming the installation of the full length of the structure, the accuracy requirement can be reduced. Therefore the use of a jack-up vessel is out of proportion. The use of pontoons is, however, considered to be a viable option. The pontoons can be kept in position by either anchoring or by using spud-poles, as presented in figure 7.5[50]. The used of tugs to aid in the lowering is presented in figure 7.6; this specific picture is for the lowering of a gravity-based wind support structure by Seatower [51].

In most lowering operation the structures are equipped with ballast tanks uniformly distributed over the full structure. This way, the lowering can be controlled easily and stable. However, the proposed structure contains a single air chamber. Assuming the requirement of being able to locally blast the structure while sinking the design should include this possibility. This could be obtained by incorporating additional blast tanks in the wall and top and bottom slaps, used temporary ballast chambers in the structure or designing the internal structure such that it can easily be used as separate ballast chambers without temporary modifications. This will not be elaborated further in this chapter.

Returning to the lowering method, the possible absence of an accurate ballasting system within the structure and the large longitudinal dimensions the lowering aided to with pontoons is assumed to be more suitable. This way the positioning can be more accurate and some additional stability can be assured by the winch cables from which the structure is hanging from the pontoon.

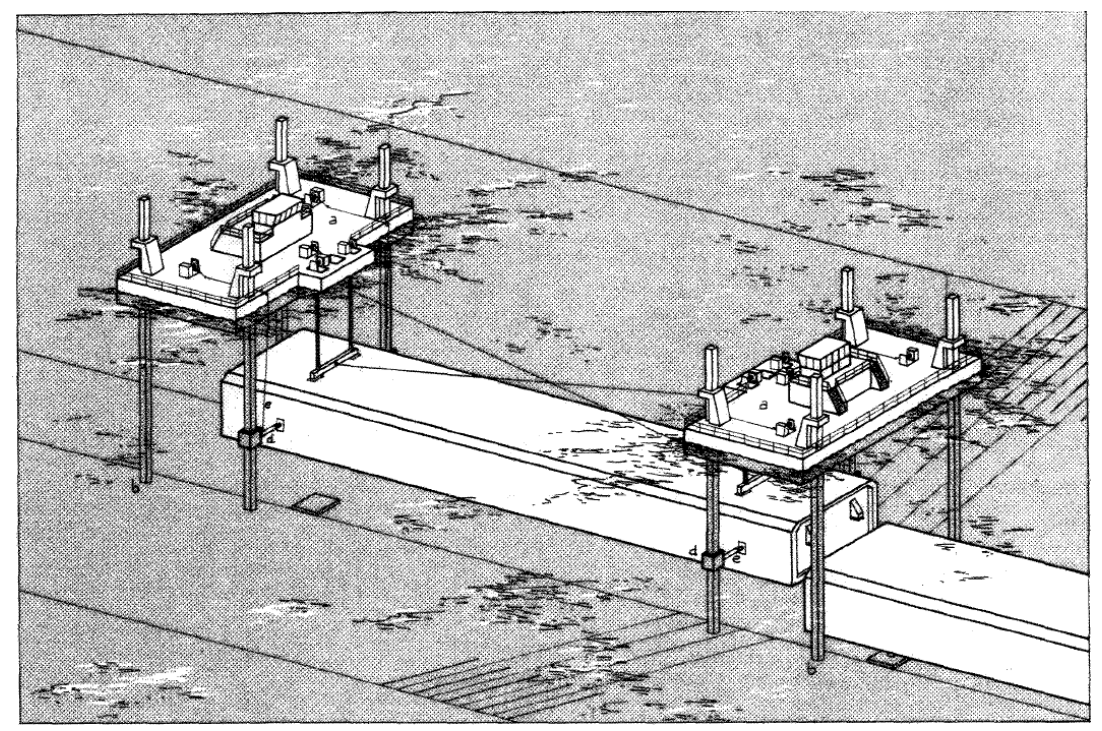

Figure 7.5: Lowering of tunnel sections using pontoons positioned by spud-poles[50]

After the installation of the turbine and auxiliary items are to be installed together with the power cables 


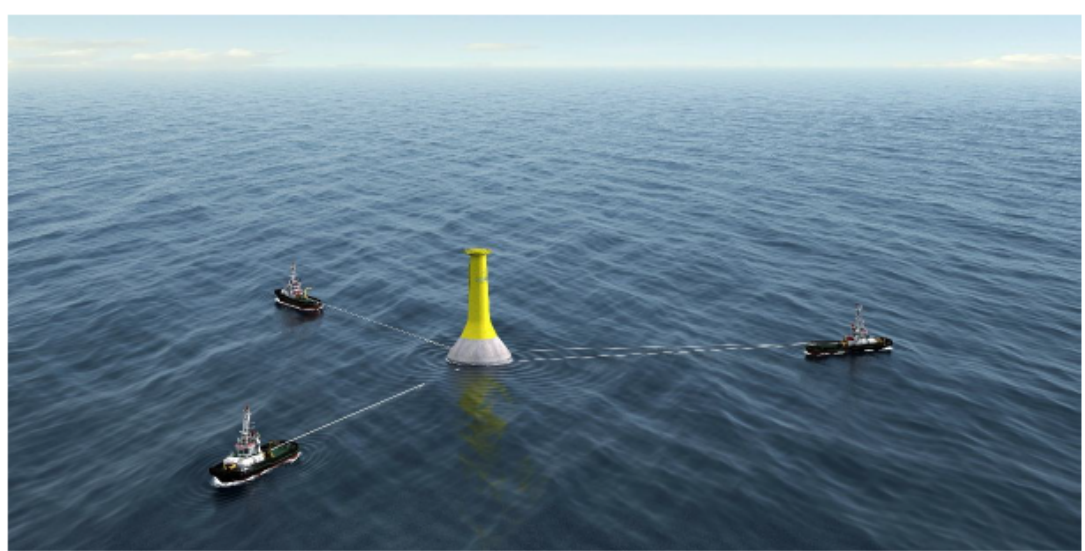

Figure 7.6: Lowering of a gravity based wind support structure aided by tugs[51]
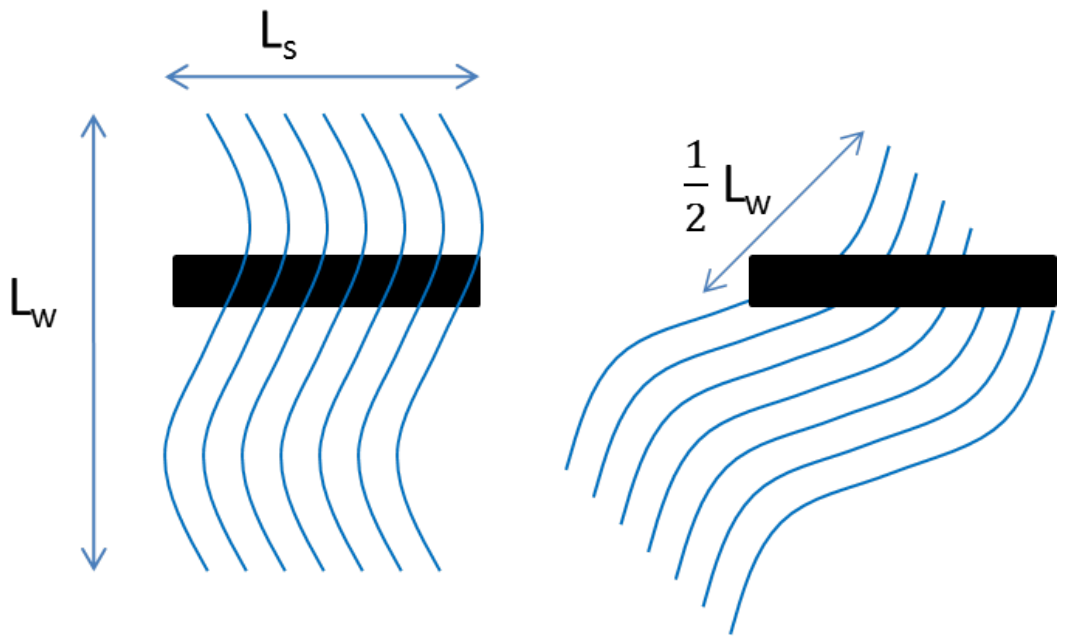

Figure 7.7: Effect of wave direction of on the pressure distribution along the structure: for $T_{w}=10 \mathrm{~s}, L_{w}=150 \mathrm{~m}$ and $L_{s}=100$

for the grid connection. Here the used of pontoons with the lowering winches can be reused, decreasing the amount of large equipment required for the operation. Further parts of the installation such as additional ballasting, anti-scour protection, underwater fastening of the turbine, grid connection etc. are not discussed further.

\subsection{Internal design}

In this section, design solutions are presented regarding the inside of the structure. The solutions are devised during a brainstorm on scaling the device. Referring to chapter 1, several full-scale OWC prototypes were designed for a capacity of $500 \mathrm{~kW}$. Therefore the design is scaled for the same design capacity to a sea state occurrence presented in figure 7.1a. In figure 7.3b a high energy extraction was found around for peak periods around 9.5 seconds and wave height between the 2 and 4 meter. The corresponding energy extraction in this region is between the 2.7 and $7.4 \frac{\mathrm{kW}}{\mathrm{m}}$, figure 7.3a. To obtain a capacity of $500 \mathrm{~kW}$, a structure width of approximately $100 \mathrm{~m}$ is required.

The increase of width introduces pressure differences along the width as a result of the wave direction. For the chosen location, the dominant wave directions were found to be west and southwest, appendix B. Assuming a wave length of $L_{w}=150$ meter corresponding to a wave period of $T_{w} \approx 10 \mathrm{~s}$, the phase difference between along the structure of $L_{s}=100 \mathrm{~m}$ is approximately half the wave length, as presented schematically in figure 7.7 .

The phase difference influences the response of the structure completely. Therefore it is suggested to divide the resonating column up into multiple independent moving columns, separated by additional walls. 


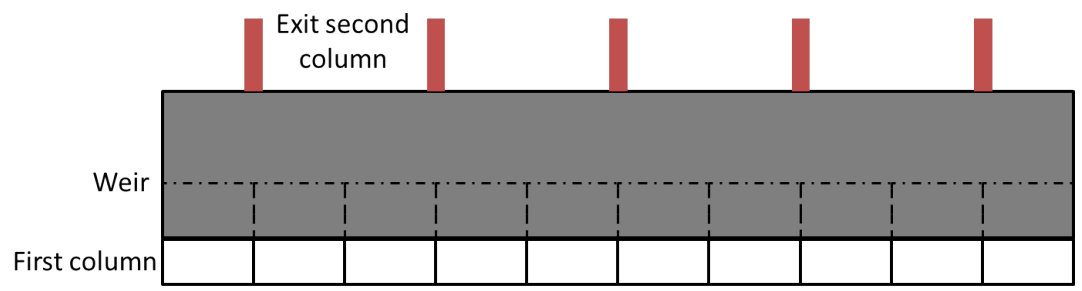

Figure 7.8: Top view
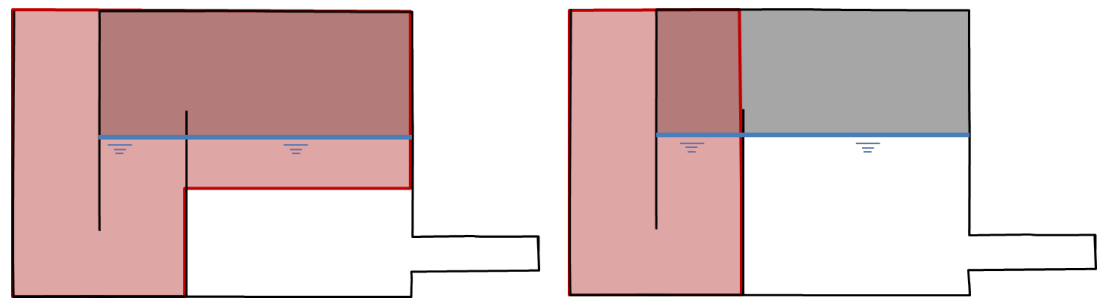

Figure 7.9: Scheme of the internal wall dividing the oscillatin columns

A schematic top view is given in figure 7.8. In the figure, it can be seen that there is a smaller amount of exit ducts than there are resonating columns. This suggests that the second column is becoming a large reservoir receiving water from the oscillating columns and discharges it through a limited amount of exit ducts, reducing the required amount of turbine.

This structure enables multiple columns to oscillate and discharge independently from each other and the pressure distribution along with the structure. Maintaining the assumption made in chapter 3 that the pressure in uniformly distributed along the mouth for $L_{w} \gg b$.

The next step is to chose how the receiving column is designed. Dependent on how far the separating walls of the oscillating column are continued into the receiving column, the individual columns are connected via the air pressure or only by the second column. If the walls are continued submerged of the free surface of the receiving column, all oscillating columns move entirely independent. The behaviour of each oscillating column is then closely described by the system modelled in this report. The second column will, however, behave differently.

However, if the separation walls are terminated above the free surface, a very large air chamber arises. The phase difference between the columns will now cause smaller relative changes of the total air volume and thus air pressure. This decreases the spring stiffness associated with the air pressure and increasing the resonance period of the oscillating column. This will, therefore, change the response of the full structure for different incoming wave directions. An alternative is to combine both approaches and obtain multiple large air chambers.

The influence of increasing the width on the performance for different wave directions is not analysed in this research. For future work, it is therefore recommended to investigate the influence of multiple oscillating columns spilling water in a single reservoir and whether the couple pneumatic stiffens can improve the annual efficiency due to its changes stiffness.

\subsection{Comparison to other wave energy converters}

In this section, the parameters above are compared to other wave energy converters. This will be done based on the found efficiency and its geometrical properties.

\subsubsection{Efficiency}

In chapter 5 a maximal efficiency of $\eta=7-12 \%$ around the natural period of the system. In this chapter, this value is converted into an annual efficiency which was found to be significantly smaller, $\eta=2.6 \%$. In [52], an overview with the annual efficiencies of different wave energy converters is presented. In Table 7.1 the efficiency range per WEC category is presented.

The found efficiency for the considered device in this research is very low compared to the efficiencies found for other systems. This can be a result of different factors, ranging from the efficiency of the power 
Table 7.1: Annual efficiency Wave energy converters [52]

\begin{tabular}{|c|c|}
\hline Category & Efficiency [\%] \\
\hline Oscillating water columns & $7-55$ \\
\hline Overtopping devices & $3-27$ \\
\hline Heaving devices & $3-40$ \\
\hline
\end{tabular}

conversions of the proposes system to the way the values are obtained for the different structures.

- The proposed system is dependent on the weir discharge, which depends on a certain weir level:

- Only a proportion of the energy potential energy in the resonating column in transferred to the second column. The lower damping in the first results in high velocities in the oscillating column, which increase the pressure losses in the system.

- The air pressure constantly accelerates and decelerated the second column, resulting in a far from stationary flow though the turbine resulting in a low efficiency of the turbine.

- The system only works when the water is allowed to flow relatively freely out of the second column. An increased resistance by the turbine decreases the mean displacement in the resonating column and the weir discharge for the same wave conditions. Therefore a large amount of kinetic energy exits the system.

- A small proportion of the potential energy at the weir level is transferred to the second column.

- The efficiency of the system decreases quickly outside for wave periods further away from the resonance period. This has a high effect on the annual efficiency with respect to the wave spectrum.

- The entrance of the resonating column is submerged to half the water depth; the pressure oscillations decrease over the depth resulting in a smaller response of the resonating column. Decreasing the depth could, therefore, improve the efficiency significantly.

- Other systems have a more direct path between the excitation and the extraction of power

- In OWC the generation is directly related between the free surface elevation inside the chamber.

- In heaving bodies, the wave pressure is directly transferred in motions which can be critically damped without interfering with the dynamics of the system

- In overtopping devices the energy is converted under relatively stationary flow, for which the high efficiency, related to hydraulic turbines, is used optimally.

- The proposed frequency distribution of wave height in [52] are more suitable for the specific devices than the distribution proposed in this research. For a frequency distribution more suited to this device, the annual efficiency could be increased significantly.

- Some of the proposed devices are still under development. The found values might be overestimated.

- The current device might not be optimized to its full potential.

\subsubsection{Geometrical}

A clear feature of the proposed wave energy converter is the submerged structure. This has the following advantages and disadvantages with respect to other wave energy converters. In this section, some advantages and disadvantages of the proposed waver energy converter are listed in compared to other converters.

Advantages:

- The only moving part is the turbine.

- The concrete structure can survive underwater for a long time; the lifetime of the project can, therefore, be elongated by replacing the turbines.

- The structure can be placed anywhere at sea and is easily scaled to capture a larger area of wave energy.

- For heaving bodies, multiple devices are required to increase the total power take-off 
- Onshore OWCs are limited to suitable coastal areas.

- Floating OWCs, overtopping devices and oscillating bodies form a large obstruction at the free surface: in both horizon pollution and possible accidental float overs.

- The structure is not being exposed to environmental conditions

- Wave impact loads do not occur

- Storm conditions are less likely to damage the structure. They are considered a danger for freesurface devices; there are records of storms destroying onshore OWC.

- The system could be integrated into an offshore wind farm without disabling the service vessels to sail freely through the wind park.

- This already a restricted area for sea traffic and optimizes the used of the ocean.

Disadvantages:

- The available energy is decreasing over the depth

- In comparison with free-surface piercing structures, the wave height is not increased due to the reflection of the wave, resulting in lower pressures at the same depth.

- The large air volume required a vast structure, in contradiction to the advantage of a more straightforward structure because of the reduced environmental loads.

- The submerged structure required underwater equipment for repairs and maintenance.

- Onshore OWCs the structure is easily accessible.

- Floating OWC and heaving and pitching bodies free-surface are accessible by vessels

- Submerged oscillating bodies can sometimes be brought to the surface by increasing its buoyancy to be more accessible by support vessels

$-$

- The device can be a threat to the marine ecosystem,

- Sea-life can be trapped inside the structure

- It disturbed the seabed and can change the habitat of sea-life

- The hydraulic turbines generate noise that can disturb the sea-life

- This is not a disadvantage for the proposed system only where all offshore structure deal with these type of threats

\subsection{Economic feasibility}

In this section, an estimation is made of the costs of the project. In the calculation, the concrete is assumed to be $1 / 3^{\text {rd }}$ of the total weight. The other $2 / 3^{\text {rd }}$ is considered ballasting sands and gravel. In [53] the cost per cubic meter concrete including reinforcement and formworks is estimated to be $426 \frac{€}{\mathrm{~m}^{3}}$ and sand $15 \frac{€}{\text { ton }}$. Multiplying the material cost with the required material results in a total cost of:

$$
\text { Cost }_{\text {total }}=\text { Cost }_{\text {concrete }}+\text { Cost }_{\text {sand }}=2.3 e^{4}+2.61 e^{3} \approx 2.61 e^{4} \frac{€}{\mathrm{~m}}
$$

In [54] the total investment cost of hydropower plants with a capacity of $500 \mathrm{~kW}$ to $1 \mathrm{MW}$ is approximately $3500 € / \mathrm{kW}$. From the cost breakdown, the equipment was found to be between 20 and $30 \%$. The turbine cost per $\mathrm{kW}$ is assumed to be:

$$
3500 \frac{€}{k W} * 25 \%=875 \frac{€}{k W}
$$

In the figure 7.3a the maximum generated power of $P_{\text {gen, } m a x} \approx 10 \frac{\mathrm{kW}}{\mathrm{m}}$. The corresponding turbine equipment cost for a rated power of $P_{\text {rated }}=10 \frac{\mathrm{kW}}{\mathrm{m}}$ is approximately $8750 \frac{€}{\mathrm{~m}}$ structure. The total cost of of the structure is becomes: 


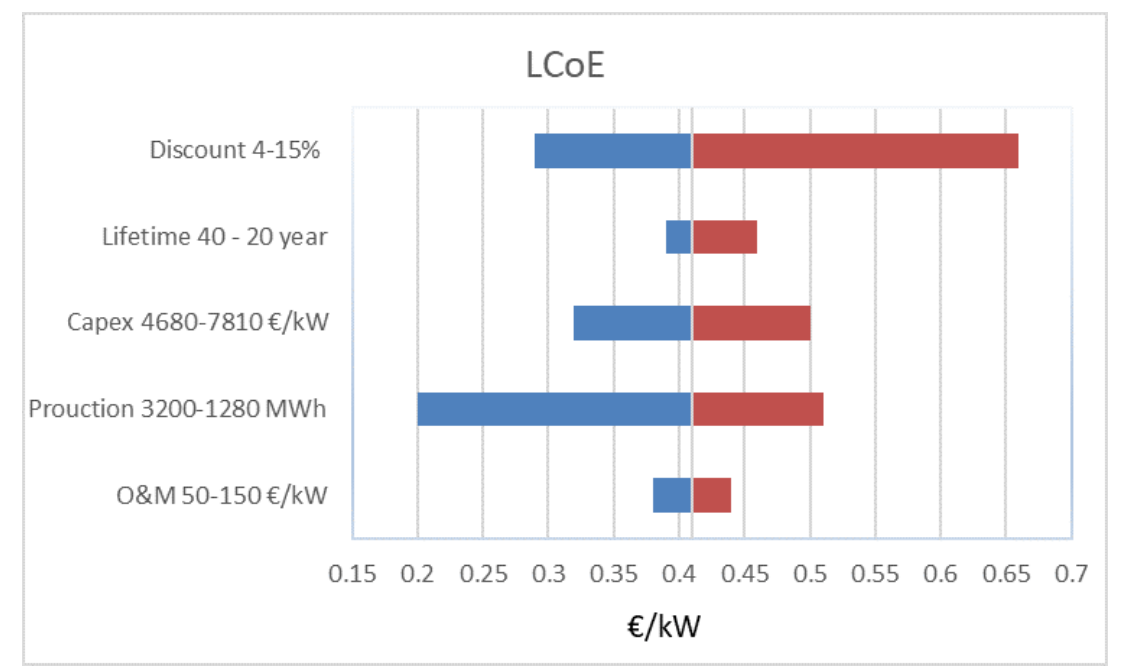

Figure 7.10: Levelises Cost of Energy Sensitivity

$$
\text { Cost }_{\text {total }} \approx 35000 \frac{€}{\mathrm{~m}}
$$

The costs of the structure an foundation per installed $\mathrm{kW}$ is now assumed as $3500 \frac{€}{\mathrm{~kW}}$. To calculate the levelised cost of electricity (LCOE) the same coast breakdown is assumed found in the offshore wind industry, presented in table 7.2[55]. The breakdown variable of wave energy in [56] showed the same results. The specific wind values are used because of the size and bottom founded nature of the presented device. The total cost is as a result of the known parameters is estimated to be 6250 euro per $\mathrm{kW}$. In recent years the average total investment cost for an offshore wind farm is $4700 € / \mathrm{kW}$, in a range of $2500-6500 € / \mathrm{kW}$.

The Operations and Maintenance (O\&M) costs are determined from the relation between wind energy on/offshore and hydro-power. The onshore wind and hydro-power both have a fixed O\&M cost of around $52 € / k W /$ year. The offshore wind average O\&M cost of $96-123 € / k W /$ year [55]. Therefore, the O\&M costs of the Neptune is estimated at $100 € / \mathrm{kW} /$ year.

From the estimated annual energy generation of $E_{\text {annual }} \approx \frac{16000 \mathrm{kWh}}{\mathrm{m}}$ the LCOE is calculated by:

$$
L C o E=\frac{\sum_{i}^{n} \frac{\text { Investment }}{(1+r)^{i}}+\sum_{i}^{n} \frac{O \& M}{(1+r)^{i}}}{\sum_{i}^{n} \frac{\text { Production }}{(1+r)^{i}}}
$$

where $r$ is the discount rate. The sensitivity graph in figure 7.10 is determined with respect to the base scenario for a discount rate of $8 \%$, a lifetime of 30 years and the above-given values for the energy production and costs. In the base case scenario, an LCoE of $0.41 \mathrm{e} / \mathrm{kW}$.

For offshore wind farms, the LCOE up to the present day is between 0.9 and $0.18 € / k W h$, with some outliers above this range[55]. This value is expected to drop below the $0.9 € / k W h$. The calculated LCoE for the base case scenario is found to be much larger than the LCoE for wind farms and is unable to compete with the current wind energy revenues.

Concluded can be that the price and performance of the current design are to low for a feasible project. However, the sensitivity graph shows that the costs are highly sensitive to an increase in production. An increase in efficiency is, therefore required to obtain a feasible business case. With little work done on the optimization of the energy transfer, a large improvement in efficiency can be expected. Additionally, a comparison of the numerical to the experimental results showed an underestimation of the response at higher periods, resulting in higher efficiencies over a larger bandwidth. This, together with a design suitable to the specific location, can also increase the production significantly. The increased efficiency directly increases the installed power, which will directly reduce the cost per MW. This is also a large sensitivity in the LCoE. For example, if the efficiency is twice the current one for the same structural dimensions: the installed power becomes $20 \mathrm{~kW} / \mathrm{m}$, the Capex reduces to approximately $3200 \mathrm{EUR} / \mathrm{kW}$ and the production increases to 3200 MWh. The base case LCoE for a discount rate of $8 \%$ and a lifetime of 30 years becomes $0.24 e / M W h$, which approaches the current wind energy industry. 
Table 7.2: Cost breakdown offshore wind [55]

\begin{tabular}{|lll|}
\hline \multicolumn{1}{|c}{ Cost breakdown offshore wind } & Percentage & $€ / \mathrm{kW}$ \\
\hline The turbine rotor and nacelle & $38 \%$ & 2380 \\
the support structure and foundations & $18 \%$ & 1130 \\
construction and installation & $19 \%$ & 1190 \\
grid connection/transmission & $13 \%$ & 813 \\
the turbine tower & $6 \%$ & 375 \\
project development & $3 \%$ & 188 \\
wind farms electrical array & $3 \%$ & 188 \\
\hline total & $100 \%$ & 6250 \\
\hline
\end{tabular}

\subsection{Conclusion}

In this chapter, an annual energy capture of $E_{\text {annual }}=15 \frac{M W h}{m}$ was found for the chosen location on the Hebrides shelf. After comparison to the total annual available wave energy at this location, an efficiency of $\eta=2.6 \%$ was found. Compared to other wave energy converters, this is efficiency in the low region. Various factors can explain this low efficiency:

- Inefficiency power transfer between the two columns

- The occurring sea-states of the chosen location is not suited for this device.

- The period bandwidth of the device is too small, with efficiencies decreasing rapidly for periods not close to the natural period

- A high amount of kinetic energy leaves the system.

- The hydraulic turbine is inefficient for the high variations of the flow found in the second column.

- The pressure losses are overestimated.

Due to the low efficiency, the investment costs per $\mathrm{kW}$ and LCOE are relatively high compared to other energy sources. However, these energy sources have been under development for decades. The proposed method has not been optimized or designed in detail yet. Several improvements, together with the key advantages of the proposed system could result in a feasible way of extracting energy.

\subsection{Discussion}

The final aim of developing a wave energy converter is to obtain a feasible economic product. This research is a new step in the development of the proposed wave energy converter. This section presented the authors view on the feasibility in both the practical and economic aspects presented in this report. This discussion will be used in the final recommendations on how to proceed in the development of the Neptune. The discussion regarding the modelling and the results are given in the previous chapters and will not be addressed here.

The biggest issue of the current design is the required size and weight of the structure. The large air volume is required to tune the natural frequency of the resonating column. However, this large volume requires a large mass of complicated mooring system to balance the buoyancy of the air chamber. Although the structural strength is not calculated, the found dimensions are considered not feasible. Two ways of reducing the air volume have been discussed in chapter 4: Reducing the area of the free surface of the resonating column or increase its length. The first will also reduce its performance, where the amplitude of the discharge decreases for the same response in displacement and velocity. It is, therefore, to be expected that the price per $\mathrm{kW}$ will not change much. The second solution is to increase the length of the resonating column. For the increased length, the total stiffness can be increased by reducing the air volume. It was found that the potential energy in the air pressure is not transferred in potential energy at the turbine efficiently. Considering both disadvantages to reduce the buoyancy, the author expects little improvements in the feasibility by both methods. The improved design must therefore mainly be focused on minimizing the costs and maximizing the efficiency, which both counteract each other and an optimal balance must be found. 



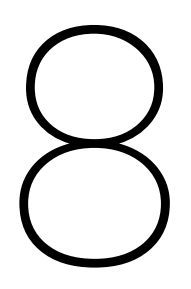

\section{Conclusion and recommendations}

The conclusions of this research and recommendations for further research are given in this chapter.

\subsection{Conclusion}

In the section below the conclusions regarding the mass transfer, the performance and the comparison to the experiments are presented.

\subsubsection{Dynamic response to periodic loads}

The numerical model results have shown that the system responds as expected. With a highly oscillating response in the first column spilling water over the weir into the second column. The resulting flow in the second column becomes uni-directional for a certain amount of weir discharge. The amount of discharge was found to be dependent on the level of the weir.

\section{Mass transfer}

As a result of the mass transfer between the columns, the maximum response periods are shifted to higher periods. The potential gravitational restoring force is transferred to the second column, reducing the stiffness. During the weir discharge, the response of the oscillating column slows down with respect to the forcing, causing resonance in higher periods. The dissipation of potential energy in the resonating column also reduces the maximum velocity response. The non-linear natural period of the resonating column can be tuned by the discharge, the volume of the air chamber and the length of the column. Changes in stiffness of the receiving second column have no relevant effect because the overall stiffness is large with respect to the resonating column.

The weir discharge is dependent on the weir level, for certain weir level the velocity in the receiving column was found to be uni-directional for all wave periods above the resonance frequency. For very low and high weir levels, the mean flow is too small to be uni-directional for the oscillation amplitudes.

The mass transfer also has associated pressures related to the momentum at the free surface and the drop of water into the second column. The influence of those pressure is negligible because they are small compared to the high pressures of the waves and internal air chamber.

\section{Power take-off}

The power take-off introduces a damping term on the second column. The amplitude of the pressure is a function of the velocity at the turbine. As a result of the high damping in the second column, the flow is obstructed. Therefore the second column discharges water at a lower rate. This causes the mean displacement to increase above its equilibrium position, and the air pressure coupling decreases the mean displacement in the resonating column. Now, for the same oscillation amplitudes in the first column, the weir discharge is reduced. An optimum is found between the extracted energy and a certain reduction of the weir discharge.

The small area of the exit pipe results in high velocities; these velocities also increase the influence of the pressure losses. The pressure losses are also dependent on the velocity, and the same effect was found by changing the pressure losses coefficient. 


\subsubsection{Performance}

The performance of the model is presented by the efficiency of the power take-off with respect to the power of the incoming waves. The maximum efficiencies to regulars waves are between 7.5 and $12 \%$. A clear peak in efficiency was found around the natural period of the structure. Below the natural period, the efficiency decreases rapidly to zero percent . At higher wave periods, the efficiency decreases a bit more gradual to values between $0 \%$ and $2 \%$ depending on the wave amplitude and weir level.

\section{Effect of the turbine diameter}

For the same weir discharge, the modelled turbine was found to generate less energy for larger diameters. Together with the less efficient large diameters, the smaller diameters were found more efficient. However, the increased damping reduced the weir discharge and eventually leads to a lower average power take-off and reversed flow at the turbine. The optimal diameter for the used system dimensions is $D_{t} 2 \mathrm{~m}$.

\section{The effect of different wave amplitudes}

The wave amplitude is linearly related to the pressure at the mouth. With the power related to the discharge over the weir, the performance of various wave amplitudes depends on the level of the weir. For small wave amplitudes, the optimal performance was found for lower weir levels than higher amplitudes. The efficiency of a regular wave with amplitudes $a_{w}=1 \mathrm{~m}$ was found to be higher with $\eta=12 \%$ for a weir level of $z_{w}=0.1 \mathrm{~m}$, while an efficiency of $\eta=7.5 \%$ for a wave with $a_{w}=3 m$ for $z_{w}=0.7 m$ is found. This can be explained by the fact that even despite the three times higher pressure at the resonating column, the height weir level results in a relatively smaller energy transfer to the second column. Despite the fact that the lower efficiency is the absolute power take-off of the higher wave amplitudes is higher because the available energy increases faster than the reduction in efficiency for increasing wave heights.

\section{Performance}

The found efficiencies for different wave heights and periods have been transformed into different irregular sea-states and extrapolated to other sea states in the annual frequency distribution of the wave height and periods. By multiplying the available power in the sea-states by the efficiencies, the annual energy productions have been calculated. The annual efficiency, at the chosen location on the Hebrides shelf, was found to lay around the $2.6 \%$. In comparison to other wave energy converters, this efficiency was found to be in the lower range of all devices in [52]. The main difference indications causing this low efficiency are: the inefficient energy transfer by the weir discharge, only gravitational energy is fully transferred, the potential energy in the air pressure is partially returned to the resonating column, the bandwidth of the response is narrow and finally the scatter diagram of the chosen location is not fully suitable for the current device.

Comparing the geometrical properties of the proposed device to other wave energy converters some major advantages have been found. First, the width of the structure can be increased easily by increasing the number of resonating column discharging water into the receiving column connected to the turbine. Increasing the power generation. Secondly, the submerges structure is not subjected to high impact loads and possible collisions, this decreasing the change of catastrophic failure. Furthermore, the device consists of a single moving part: the turbine. The main structure, which collects the wave energy, is therefore more durable. Lastly, the device can be installed in shallow to moderate depth seas, and is not limited to specific coastal area's. It could also be easily combined with existing offshore wind farms, without interfering with the support vessels.

However, the structure also has several weaknesses. A large air chamber is required to obtain resonance and to avoid buoyancy a heavy counterweight or anchoring is required. This reduces the convenience of a simplified structure due to the reduces environmental loads. The submerged structure is also poorly accessible for repairs and maintenance, increasing the operations and maintenance costs.

The low efficiency and high cost result in a high $L C o E$ value of $L C o E=0.41 \mathrm{e} / \mathrm{kW}$. From this value and the complexity of the structure it is concluded that the device is not feasible in current form. To obtain a feasible device major improvements are required. From the sensitivity analysis the costs per $\mathrm{kW}$ and production rate could improve the concept significantly. It is expected that it is possible to improve the LCoE value significantly. However, it is not expected to be able to compete with the currently available renewable energy sources.

\subsubsection{Validation}

The simulation for the small diameter exit pipe of the second column the numerical response underestimates the resonance peaks compared to the experimental results. A clear difference was found for the amplitudes of 
the velocities. The numerical results the minimum velocity was is underestimated for both weir levels, while the mean and maximum velocity peaks are closer to the experimental results. The simulation for the larger exit pipe the resonance peaks was closer to the experimental results. Also, the minimum values were found to be approximated more closely. The main difference between the two results was the inertia of the second column. Where the inertia of the smaller diameter was chosen larger because of the high area ratio between the free surface and exit pipe of the second column. Additional a discrepancy was found in the minimum velocity at low periods, where the minimum flow is expected to negative, implying a calibration error.

In both cases, the peak periods was underestimated by the numerical model; however, in the smaller diameter simulation, a more significant difference was observed. This is likely caused by an underestimation of the length or added length of the resonating column, or an overestimation of the air pressure stiffness or lower weir discharge.

Apart from the different resonance peaks, the experimental results were found to have a larger response over a wider bandwidth, with the slope to higher periods less steep than the numerical results. This was mainly observed for the higher weir levels. It is presumed that this is caused by an underestimation of the motion of the first column in the numerical model due to either smaller wave loads due to the overestimated pressure losses in one of the columns.

\subsection{Recommendations}

For a feasible device, major improvements in the performance are required, together with a workable design. The recommendation for further research to achieve those improvements are given below.

First of all, the numerical model must be improved. Reliable hydrodynamic forces and coefficient have to be obtained for the complex structure. Together with a verified polytropic coefficient $\gamma$ and a more realistic turbine model. The hydrodynamic performance of the simple model must be verified by further experiments and/or a computational fluid dynamics model. Here the viscous and turbulence effect can be analysed better, and the corresponding pressure loss coefficients can be determined. The model also has to be capable of simulating the response to wave spectra, instead of only regular waves with a single wave period.

Next, the performance of the device itself has to be improved. Starting with the efficiency of the power take-off from waves. An improve the energy transfer between the columns, it is expected to be obtained by increasing the influence of the gravitational stiffness compared to the air pressure stiffness. This way, the efficiency to single wave periods and the bandwidth of the response will be improved.

Further, an advanced design of the structure is required. To generate sufficient energy, the width of the structure must be increased. Subsequently, the effect of an array of multiple oscillating water column spilling water into a single discharging reservoir must be analysed. The design modifications must go parallel to a cost reduction of the structure. 



\section{Bibliography}

[1] G. Mørk, S. Barstow, A. Kabuth, and M. T. Pontes, "Assessing the global wave energy potential," in Proceedings of International Conference on Ocean, Offshore Mechanics and Arctic Engineering, no. 29, (Shanghai), pp. 1-8, ASME, 2010.

[2] D. J. Carey and M. Pemberton, “A fully submerged omni-directional oscillating Denis Carey," in Instutude of marine engineers renewable energy conference, (Newcastly), 2001.

[3] J. Lighthill, "Two-dimensional analyses related to wave-energy extraction by submerged resonant ducts," Journal of Fluid Mechanics, vol. 91, no. 2, pp. 253-317, 1979.

[4] G. F. Knott and J. O. Flower, "Wave-tank experiments on an immersed vertical circular duct," Journal of Fluid Mechanics, vol. 100, no. 2, pp. 225-237, 1980.

[5] G. F. Knott and J. O. Flower, "Simulation studies of basic non-linear effects of wave-energy conversion by an overtopping water column,” Energy Conversion, vol. 19, pp. 59-69, 1978.

[6] L. Rusu and F. Onea, "The performance of some state-of-the-art wave energy converters in locations with the worldwide highest wave power," Renewable and Sustainable Energy Reviews, vol. 75, no. November 2016, pp. 1348-1362, 2017.

[7] A. F. Falcão and J. C. Henriques, "Oscillating-water-column wave energy converters and air turbines: A review," Renewable Energy, vol. 85, pp. 1391-1424, 2016.

[8] P. Mccullen, A. Cle, A. Fiorentino, F. Gardner, K. Hammarlund, G. Lemonis, T. Lewis, K. Nielsen, H. Christian, and T. Thorpe, "Wave energy in Europe : current status and perspectives," Renewable and Sustainable Energy Reviews, vol. 6, pp. 405-431, 2002.

[9] K. Gunn and C. Stock-williams, "Quantifying the global wave power resource," Renewable Energy, vol. 44, pp. 296-304, 2012.

[10] J. Cruz, Ocean Wave Energy: Current Status and Future Perspectives. Springer-Verlag Berlin Heidelberg, 1 ed., 2008.

[11] L. Holthuijsen, Waves in oceanic and coastal waters. Cambridge Uniersity Press, 2007.

[12] F. D. O. Falca, "Wave energy utilization : A review of the technologies," Renewable and Sustainable Energy Reviews, vol. 14, pp. 899-918, 2010.

[13] P. Boccotti, “On a new wave energy absorber,” Ocean Engineering, vol. 30, no. 9, pp. 1191-1200, 2003.

[14] P. Beirão and D. Valerio, “Thermal modelling of the Archimedes Wave Swing,” in JSC, no. January, 2006.

[15] W. Knapp, C. Böhm, J. Keller, W. Rohne, and R. Schilling, “Turbine development for the Wave Dragon wave energy converter,” tech. rep., Technische Universität München, München, 2003.

[16] M. E. Mccormick, “A modified linear analysis of a wave-energy convertion buoy,” vol. 3, no. 1975, pp. 133-144, 1976.

[17] M. E. Mccormick, “Analysis of a wave energy conversion buoy,” Journal of Hydronautics, vol. 8, no. 3, pp. 77-82, 1974.

[18] D. V. Evans, “The Oscillating Water Column Wave-energy Device,” pp. 423-433, 1978.

[19] J. Falnes, “Ocean waves and oscillating systems,” in Ocean waves and oscillating systems, ch. 7, pp. 225261, Cambridge Uniersity Press, 2002 ed., 202. 
[20] B. D. V. Evans, “Wave-power absorption by systems of oscillating surface pressure distributions,” vol. 114, 1982.

[21] G. F. Knott and J. O. Flower, "Measurement of energy losses in oscillatory flow through a pipe exit," $A p$ plied Ocean Research, vol. 2, no. 4, pp. 155-164, 1980.

[22] M. Ohmi, M. Iguchi, and I. Urahata, “Flow patterns and frictional losses in oscillating pipe flow,” JSME, vol. 25, no. 202, pp. 536-543, 1982.

[23] G. F. Knott and M. R. Mackley, "On eddy motions near plates and ducts, induced by water waves and periodic flows,” Mathematical and Physcal science, vol. 294, no. 1412, p. 5990623, 1980.

[24] B. A. J N A Sarmento and A. F. De, "Wave generation by an oscillating surface-pressure and its application in wave-energy extraction,” Journal of Fluid Mechanics, vol. 150, pp. 467-485, 2017.

[25] O. Falca and P. Justino, “OWC wave energy devices with air flow control,” vol. 26, pp. 1275-1295, 1999.

[26] S. D. J. Costa, A. Sarmento, F. Gardner, P. Beirão, and A. Brito-melo, "Time domain model of the archimedes wave swing wave energy converter," in 6th European wave and tidel Energy Conference., (Glasgow), pp. 91-97, 2004.

[27] C. Kpordze and C. Warnick, “Experience curves for modern low-head Hydroelecric turbines.” 1983.

[28] S. Arnaltes and J. C. Burgos, "Control of Permanent-Magnet Generators Applied to Variable-Speed WindEnergy Systems Connected to the Grid,” vol. 21, no. March, pp. 130-135, 2006.

[29] F. Gonzales-Longatt, P. Wall, and V. Terzija, "A Simplified Model for Dynamic Behavior of Permanent Magnet Synchronous Generator for Direct Drive Wind Turbines,” no. May 2014, 2011.

[30] F. Dias and E. O. Tuck, "Weir flows and waterfalls," Journal of Fluid Mechanics, vol. 230, pp. 525-539, 1991.

[31] S. Czitrom, R. Godoy, E. Prado, P. Pérez, and R. Peralta-Fabi, "Hydrodynamics of an oscillating water column seawater pump,” Ocean Engineering, vol. 27, no. 11, pp. 1181-1198, 2000.

[32] G. Malara and F. Arena, "Analytical modelling of an U-Oscillating Water Column and performance in random waves,” Renewable Energy, vol. 60, pp. 116-126, 2013.

[33] P. Kundu and Cohen I.M., Fluid Mechanic. Elsevier, fourth edition ed., 1987.

[34] K. M. Signh, “Computational fluid dynamics: Mathemetical Modeling,” 2012.

[35] W. Cummins, “The impulse response fuctnion and ship motions," tech. rep., David Taylor model basin, 1962.

[36] G. F. Knott and J. O. Flower, "Wave-tank experiments on an immersed parallel-plate duct," Journal of Fluid Mechanics, vol. 90, no. 2, pp. 327-336, 1979.

[37] G. Malara, R. P. Gomes, F. Arena, J. C. Henriques, L. M. Gato, and A. F. Falcão, "The influence of threedimensional effects on the performance of U-type oscillating water column wave energy harvesters," Renewable Energy, vol. 111, pp. 506-522, 2017.

[38] A. Kotowski, H. Szewczyk, and W. Ciezak, "Entrance loss coefficients in pipe hydraulic systems," Environment Protection Engineering, vol. 37, no. 4, pp. 105-117, 2011.

[39] N. Kikuchi, Finite element methods in mechanics. Ann Arbor: Cambridge Uniersity Press, 1 ed., 1986.

[40] FlyToMap, “Fly to Map,” 2018.

[41] FugroGEOS, "Wind and wave frequency distributions for sites around the British Isles," tech. rep., Fugro GEOS, 2001.

[42] Univerity of Strathclyde, “Kelvin Hydrodynamics Laboratory.” 
[43] S. Stripling and D. Greaves, “Testing of the "Neptune " Wave Energy Conversion Device Technical Note prepared for Land and Water Group,” Tech. Rep. August, Coast Labrotory, Plymouth University, Plymouth, 2013.

[44] C. Perez-Collazo, D. Greaves, and G. Iglesias, "A novel hybrid wind-wave energy converter for jacketframe substructures,” Energies, vol. 11, 22018.

[45] W. Koo and M.-H. Kim, "A time-domain simulation of an oscillating water column with irregular waves," Ocean Systems Engineering, vol. 2, pp. 147-158, 72013.

[46] M. Voorendt, W. Molenaar, and K. Bezuyen, Lecture notes VT3330: Hydraulic Structures: Caissons. Delft: Department of Hydraulic Engineering Faculty of Civil Engineering, TU Delft, february 2011 ed., 2011.

[47] Renewable First, “Hydropower," 2019.

[48] M. D. Esteban, B. Couñago, J. S. López-Gutiérrez, V. Negro, and F. Vellisco, "Gravity based support structures for offshore wind turbine generators: Review of the installation process,” 122015.

[49] I. Ruiz de Temiño Alonso, "GRAVITY BASE FOUNDATIONS FOR OFFSHORE WIND FARMS MARINE OPERATIONS AND INSTALLATION PROCESSES,” tech. rep., Master in European Construction Engineering, 2013.

[50] R. Lunniss and J. Baber, “Immersed tunnels,” Immersed Tunnels, pp. 1-487, 101978.

[51] OffshoreWind.biz, “Norway: Seatower Raises Funds with Electranova Capital,” 2012.

[52] A. Babarit, "A database of capture width ratio of wave energy converters," Renewable Energy, vol. 80, pp. 610-628, 82015.

[53] A. v. d. Horst, "CT 4170 Construction Technology of Civil Engineering Projects Lecture notes," No. V6.0, ch. 3, pp. 30-44, Delft: TU Delft, 2018.

[54] International Renewable Energy Agency, "Renewable Energy Technologies: Hydropower cost analysis series," Renewable Energy Technologies: Cost Analysis Series, vol. 1, no. 3/5, p. 44, 2012.

[55] IRENA, "Renewable Power Generation Costs in 2017," tech. rep., International Renewable Energy Agency, 2018.

[56] D. Magagna, “The European Commission’s science and knowledge service,” 2018. 



\section{List of Figures}

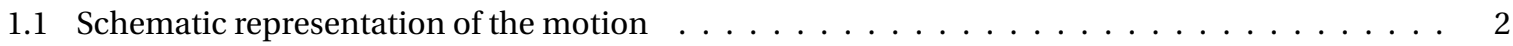

1.2 Scale prototype Neptune Energy LTD, in testing facilities Glasgow 2008 . . . . . . . . . . . 2

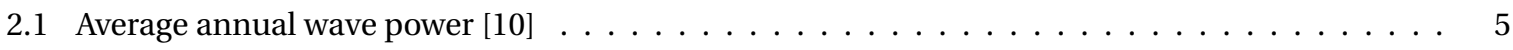

3.1 Drawing full scale Neptune, as design of Neptune Energy LTD. . . . . . . . . . . . . . . . 11

3.2 Cross-section of the submerged Oscillating water column, symbols denote the geometrical char-

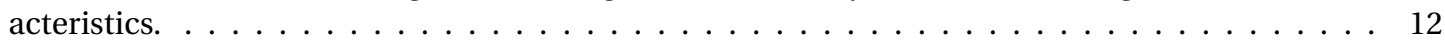

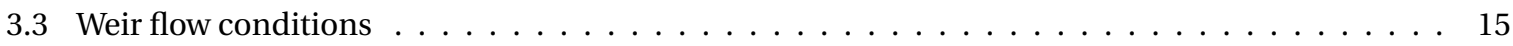

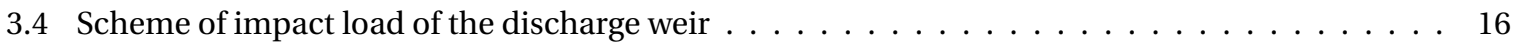

3.5 Radiation damping coefficient D, for mouth-upwards duct, calculated on two dimensional theory as a function of the width-wavelength ratio $n$ for different values of the depth-wavelength ratio $\frac{h}{\lambda}$. This coefficient $D_{r}$, is defined so that the energy radiated in new surface waves takes the form (3.73) per unit breadth, when the duct volume flow per unit breadth oscillates with

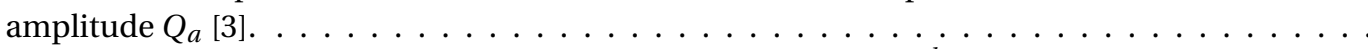

3.6 Added-mass curves in the mouth-upwards case, giving the ratio $\frac{l}{n \lambda}$ of the effective added length of duct to the width $n h$ of the duct mouth, as a function of the ratio $\frac{h}{\lambda}$ of mouth depth to wave-

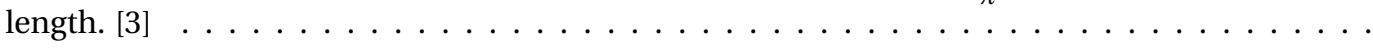

3.7 Relation between turbine power and water velocity for different rotational speeds according to empirical relations from Bulb turbines [27]; a turbine diameter of $2 \mathrm{~m}$ is used. . . . . . . . . . . .

4.1 Water depths Hebrides Shelf $[40] \ldots \ldots \ldots \ldots \ldots \ldots \ldots \ldots \ldots$

4.2 Location wave data Hebrides Shelf $[41] \ldots \ldots \ldots \ldots \ldots \ldots \ldots \ldots \ldots \ldots$

4.3 Dependence of natural period on the column areas met meter with . . . . . . . . . . . 35

5.1 Wave length (left) and wave number (right) for different wave periods for a depth of $d=40 \mathrm{~m}$. . 38

5.2 Wave pressures on column 1 (left) and column 2 (right) $\ldots \ldots \ldots \ldots \ldots \ldots$

5.3 Hydrodynamic coefficients: Added length $L_{a}$ (left) and Radiation damping $D_{r}$ (right) $\ldots \ldots \ldots$

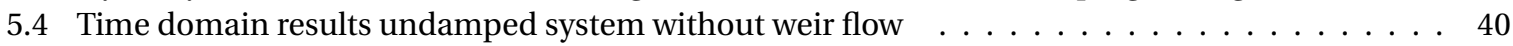

5.5 Time domain results damped system without weir flow $\ldots \ldots \ldots \ldots$. . . . . . . . . . 40

5.6 Time domain results undamped system without weir flow start of the simulation . . . . . . . . 41

5.7 Comparison non-linear undamped system with linear frequency response function, . . . . . . 41

5.8 Comparison non-linear damped system with linear frequency response function . . . . . . . . 41

5.9 Maximum displacement and discharge of Column 1(solid lines) and Column 2 (dashed line) for different weir levels . . . . . . . . . . . . . . . . . . . . . . . . . 42

5.10 Minimum displacement and discharge of Column 1 (solid lines) and Column 2 (dashed line) for

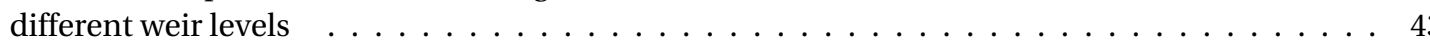

5.11 Mean displacement and discharge of Column 1(solid lines) and Column 2 (dashed line) for different weir levels . . . . . . . . . . . . . . . . . . . . . . . . . 44

5.12 Mean displacement and discharge of undamped model of Column 1(solid lines) and Column 2 (dashed line) for different weir levels . . . . . . . . . . . . . . . . . . . . . . . . 44

5.13 Damped time domain steady state response for a wave amplitude of $2 \mathrm{~m} \ldots \ldots \ldots$

5.14 Maximum air pressure (solid line) and maximum wave pressure on column 1 (Dashed line) $\quad . \quad 45$

5.15 Mean power for different rotation speeds for $D_{t}=2 m \ldots \ldots \ldots \ldots \ldots$. . . . . . . . 47

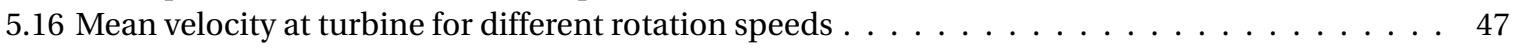

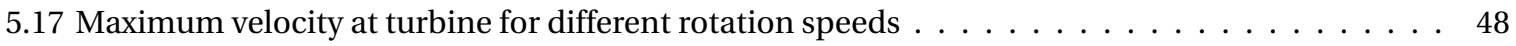

5.18 Minimum velocity at turbine for different rotation speeds . . . . . . . . . . . . . . . . 48

5.19 Maximum discharge first column for different rotation speeds . . . . . . . . . . . . . . 48

5.20 Mean power for different rotation speeds for $D_{t}=1.5 m \ldots \ldots \ldots \ldots$ 
5.21 Mean power for different rotation speeds for $D_{t}=1.75 m \ldots \ldots \ldots \ldots \ldots \ldots$

5.22 Mean power for different rotation speeds for $D_{t}=2.25 m \ldots \ldots \ldots \ldots \ldots$

5.23 Mean power for different rotation speeds for $D_{t}=2.5 m \ldots \ldots \ldots \ldots \ldots \ldots$

5.24 Available power per meter wave crest of regular incoming waves with different amplitudes $\quad \ldots \quad 51$

5.25 Power efficiency for varying wave amplitudes at a weir level of $z_{w}=0.5 \mathrm{~m} \ldots \ldots \ldots$. . . . . . . . . . . .

5.26 Power efficiency for varying wave amplitudes at a weir level of $z_{w}=0.3 m \ldots \ldots$. . . . . . 52

5.27 Power efficiency for varying wave amplitudes at a weir level of $z_{w}=0.7 \mathrm{~m} \ldots \ldots \ldots$

5.28 Power efficiency for varying wave amplitudes at a weir level of $z_{w}=1 \mathrm{~m} \ldots \ldots \ldots 3$

6.1 Neptune building drawing $1 / 20^{\text {th }}$ scale model $\ldots \ldots \ldots \ldots \ldots$. . . . . . . . . . . . 55

6.2 Time domain response experiments, for $z_{w}=50 \mathrm{~mm}$ and $T_{\text {measured }}=2.1 \mathrm{~s}$ and an exit pipe diameter of $D=195 \mathrm{~mm} \ldots \ldots \ldots \ldots \ldots \ldots \ldots \ldots$

6.3 Mean velocity for different wave periods for a weir level of $z_{w}=50 \mathrm{~mm}$ and an exit pipe diameter

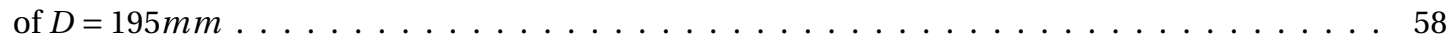

6.4 Maximum velocity for different wave periods for a weir level of $z_{w}=50 \mathrm{~mm}$ and an exit pipe diameter of $D=195 \mathrm{~mm}$ and an exit pipe diameter of $D=195 \mathrm{~mm} \ldots \ldots \ldots \ldots$

6.5 Minimum velocity for different wave periods for a weir level of $z_{w}=50 \mathrm{~mm}$ and an exit pipe

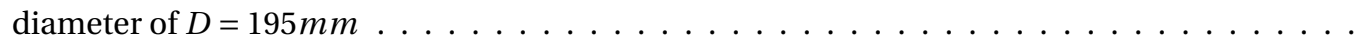

6.6 Mean velocity for different wave periods for a weir level of $z_{w}=75 \mathrm{~mm}$ and an exit pipe diameter

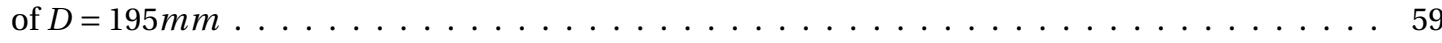

6.7 Maximum velocity for different wave periods for a weir level of $z_{w}=75 \mathrm{~mm}$ and an exit pipe diameter of $D=195 \mathrm{~mm} \ldots \ldots \ldots \ldots \ldots \ldots \ldots \ldots$

6.8 Minimum velocity for different wave periods for a weir level of $z_{w}=75 \mathrm{~mm}$ and an exit pipe

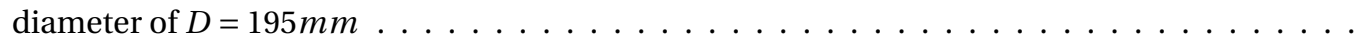

6.9 Mean velocity for different wave periods for a weir level of $z_{w}=35 \mathrm{~mm}$ and an exit pipe diameter of $D=245 \mathrm{~mm} \ldots \ldots \ldots \ldots \ldots \ldots \ldots \ldots \ldots \ldots \ldots \ldots$

6.10 Mean velocity for different wave periods for a weir level of $z_{w}=50 \mathrm{~mm}$ and an exit pipe diameter of $D=245 \mathrm{~mm} \ldots \ldots \ldots \ldots \ldots \ldots \ldots \ldots \ldots \ldots \ldots \ldots$

6.11 Mean velocity for different wave periods for a weir level of $z_{w}=75 \mathrm{~mm}$ and an exit pipe diameter

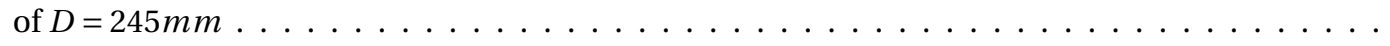

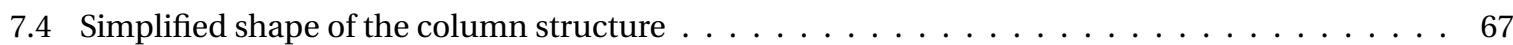

7.5 Lowering of tunnel sections using pontoons positioned by spud-poles[50] . . . . . . . . . . 69

7.6 Lowering of a gravity based wind support structure aided by tugs $[51] \ldots \ldots$. . . . . . . . . 70

7.7 Effect of wave direction of on the pressure distribution along the structure: for $T_{w}=10 \mathrm{~s}, L_{w}=$

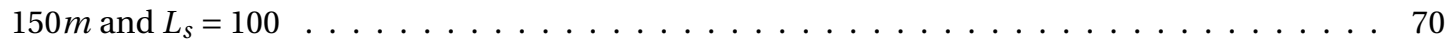

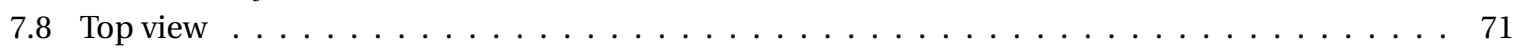

7.9 Scheme of the internal wall dividing the oscillatin columns $\ldots \ldots \ldots \ldots \ldots \ldots$

7.10 Levelises Cost of Energy Sensitivity . . . . . . . . . . . . . . . . . . . . . . . . . . . 74

A.1 Maximum velocity through turbine for different rotation speeds . . . . . . . . . . . . . 91

A.2 Maximum velocity through turbine for different rotation speeds . . . . . . . . . . . . . . . . 92

B.1 Distribution Spectral peak periods around the british Isles: Figure 2 in [41] . . . . . . . . . . . 93

B.2 Distribution Spectral peak periods around the british Isles: Figure 2 in [41] . . . . . . . . . . . . . 94

B.3 Distribution Spectral peak periods around the british Isles: Figure 2 in [41] . . . . . . . . . . . . 94

B.4 Distribution Spectral peak periods around the british Isles: Figure 2 in [41] . . . . . . . . . . . . 95

C.1 Maximum velocity at turbine for $D_{t}=1.5 m \ldots \ldots \ldots \ldots \ldots \ldots$

C.2 Minimum velocity at turbine for $D_{t}=1.5 m \ldots \ldots \ldots \ldots \ldots \ldots \ldots \ldots$

C.3 Mean velocity at turbine for $D_{t}=1.5 m \ldots \ldots \ldots \ldots \ldots \ldots \ldots \ldots$

C.4 Mean power for different rotation speeds for $D_{t}=1.5 m \ldots \ldots \ldots \ldots \ldots \ldots$

C.5 Maximum velocity at turbine for $D_{t}=1.75 m \ldots \ldots \ldots \ldots \ldots \ldots$

C.6 Minimum velocity at turbine for $D_{t}=1.75 m \ldots \ldots \ldots \ldots \ldots \ldots \ldots$

C.7 Mean velocity at turbine for $D_{t}=1.75 m \ldots \ldots \ldots \ldots \ldots \ldots \ldots$

C.8 Mean power for different rotation speeds for $D_{t}=1.75 m \ldots \ldots \ldots \ldots \ldots \ldots$

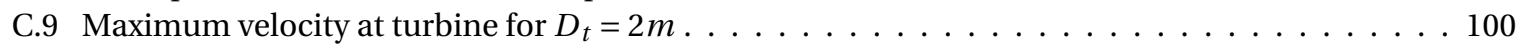


C.10 Minimum velocity at turbine for $D_{t}=2 m \ldots \ldots \ldots \ldots \ldots \ldots \ldots$

C.11 Mean velocity at turbine for $D_{t}=2 m \ldots \ldots \ldots \ldots \ldots \ldots \ldots \ldots \ldots \ldots$

C.12 Mean power for different rotation speeds for $D_{t}=2 m \ldots \ldots \ldots \ldots \ldots \ldots$

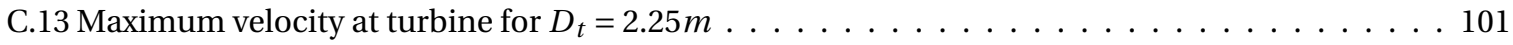

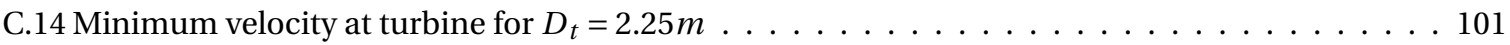

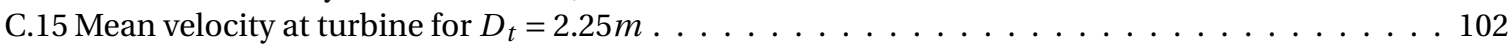

C.16 Mean power for different rotation speeds for $D_{t}=2.25 m \ldots \ldots \ldots \ldots \ldots$. . . . . . . . . 102

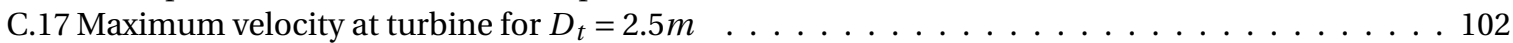

C.18 Minimum velocity at turbine for $D_{t}=2.5 m \ldots \ldots \ldots \ldots \ldots \ldots$

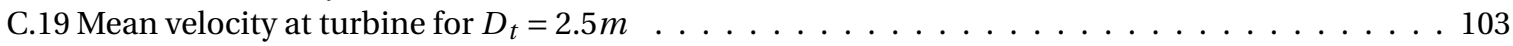

C.20 Mean power for different rotation speeds for $D_{t}=2.5 m \ldots \ldots \ldots \ldots \ldots$

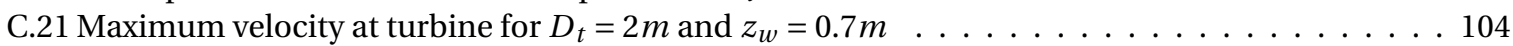

C.22 Minimum velocity at turbine for $D_{t}=2 m$ and $z_{w}=0.7 m \ldots \ldots \ldots \ldots$. . . . . . . 104

C.23 Mean velocity at turbine for $D_{t}=2 m$ and $z_{w}=0.7 m \ldots \ldots \ldots \ldots \ldots \ldots$

C.24 Mean power for different rotation speeds for $D_{t}=2 m$ and $z_{w}=0.7 m \ldots \ldots \ldots$

D.1 Hand written notes settings and average results Glasgow testing: part $1 \ldots \ldots$. . . . . . . . . 108

D.2 Hand written notes settings and average results Glasgow testing: part $2 \ldots \ldots$. . . . . . . . 109 



\section{List of Tables}

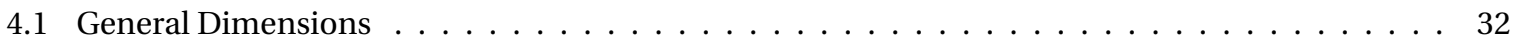

4.2 Dimensions initial frequency analysis per meter width $\ldots \ldots \ldots \ldots \ldots \ldots \ldots$

4.3 Dimensions initial frequency analysis for a structure width of 10 meter, corresponding to 3.2 . . 35

5.1 The maximum and minimum pressures related to the weir discharge in $\mathrm{Pa} \ldots \ldots \ldots 4$

5.2 Maximum mean power output for different wave amplitudes and optimal weir level $\ldots$. . . . . 52

6.1 Dimensions initial frequency analysis for a structure width of 10 meter, corresponding to figure

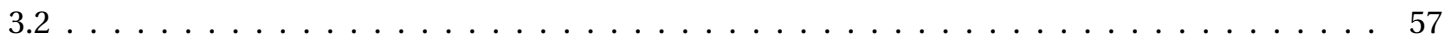

7.1 Annual efficiency Wave energy converters $[52] \ldots \ldots \ldots \ldots \ldots \ldots \ldots \ldots \ldots \ldots$

7.2 Cost breakdown offshore wind $[55] \ldots \ldots \ldots \ldots \ldots \ldots \ldots \ldots$ 



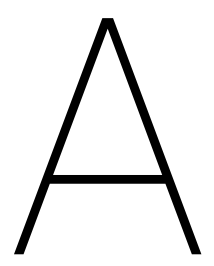

\section{Turbine Characteristics}

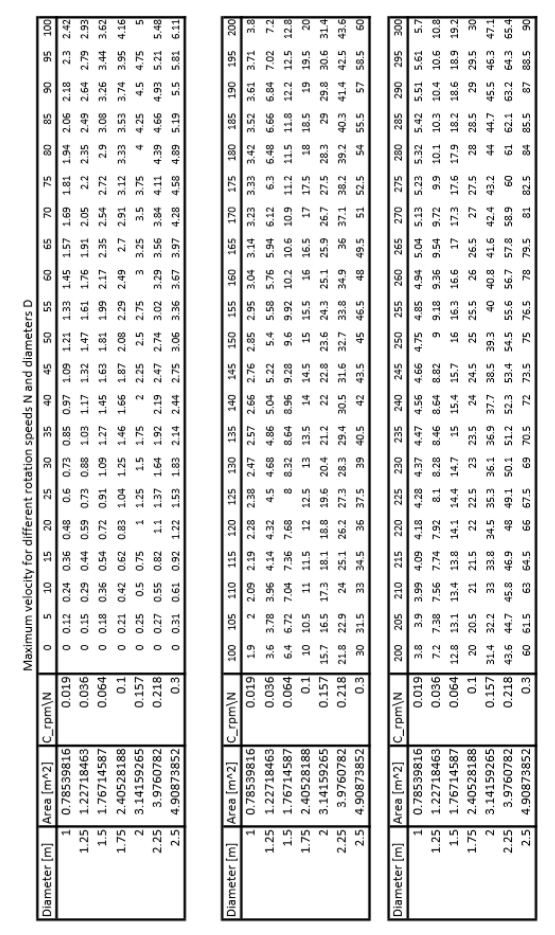

Figure A.1: Maximum velocity through turbine for different rotation speeds 


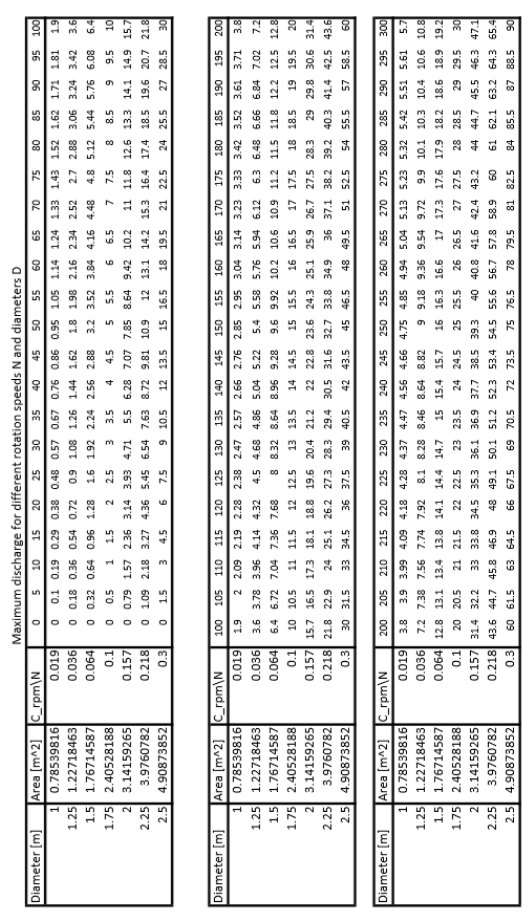

Figure A.2: Maximum velocity through turbine for different rotation speeds 


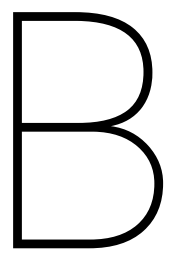

\section{Appendix A: Wave data Hebrides Shelf}

In this appendix the graphs and tables related to the wave climate of location NEXT Grind Point 15609, in [41] at the Hebrides Shelf along the UK coast, are presented.

Figure 2 - Marginal Distributions of Spectral Peak Period Around the British Isles

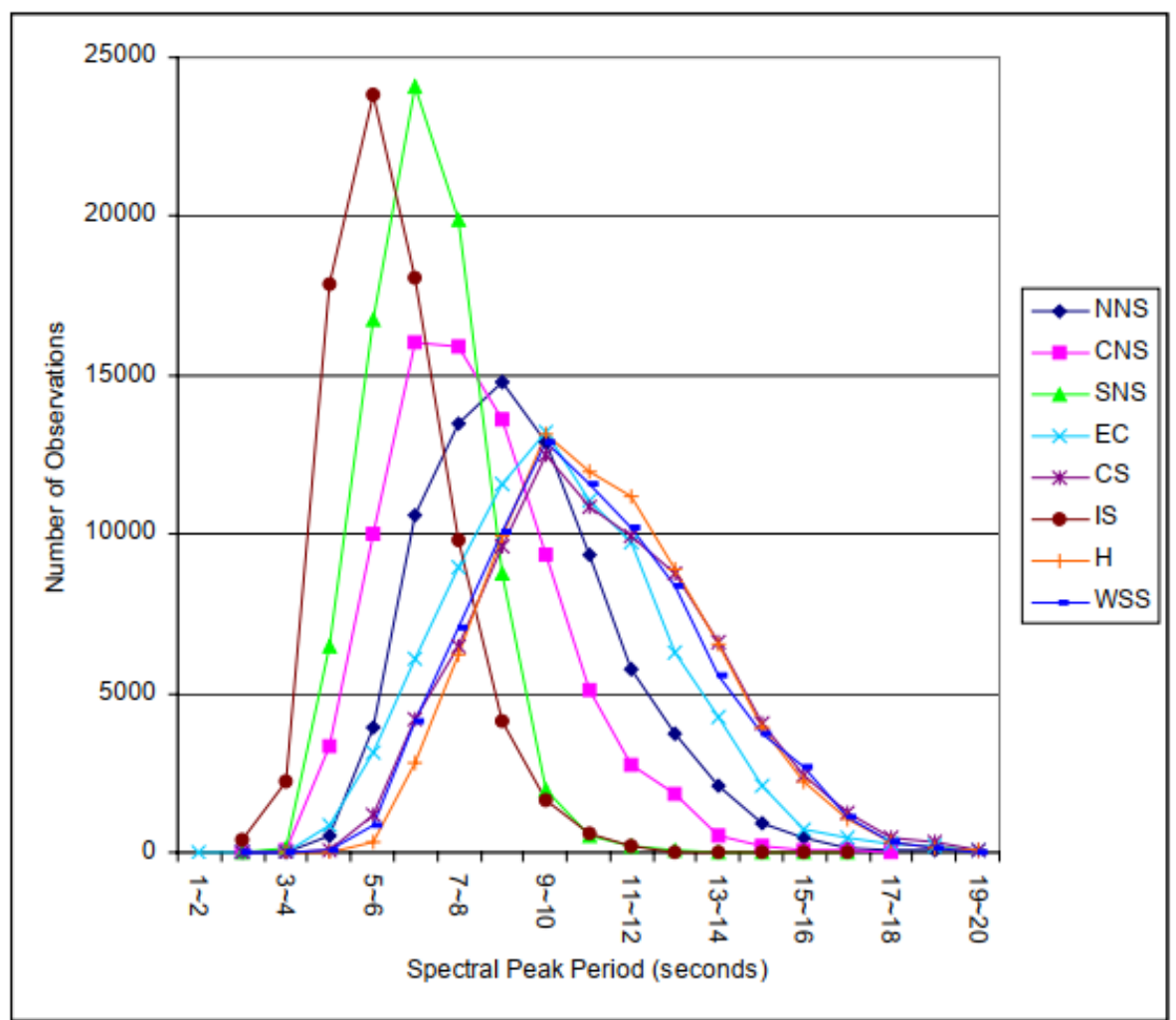

NNS = Northern North Sea; CNS = Central North Sea; SNS = Southern North Sea; EC = English Channel; CS = Celtic Sea; IS = Irish Sea; $\mathrm{H}=$ Hebrides; WSS = West Shetland Shelf

Figure B.1: Distribution Spectral peak periods around the british Isles: Figure 2 in [41] 


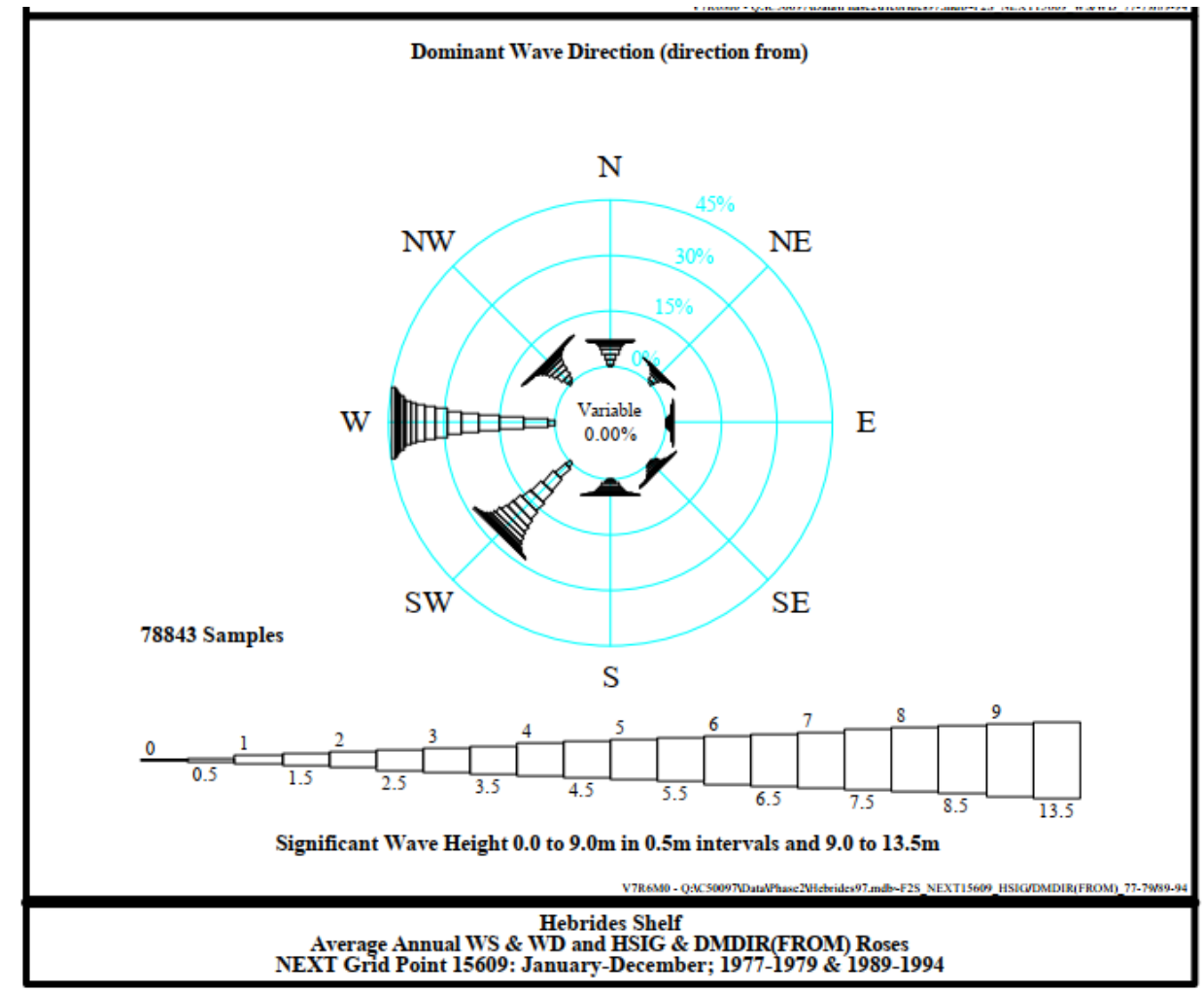

Figure B.2: Distribution Spectral peak periods around the british Isles: Figure 2 in [41]

Significant Wave Height - Percentage Exceedence Distribution

\begin{tabular}{|c|cccccccccccc|c|}
\hline $\mathrm{m}$ & Jan & Feb & Mar & Apr & May & Jun & Jul & Aug & Sep & Oct & Nov & Dec & Year \\
\hline 0.0 & 100.00 & 100.00 & 100.00 & 100.00 & 100.00 & 100.00 & 100.00 & 100.00 & 100.00 & 100.00 & 100.00 & 100.00 & 100.00 \\
0.5 & 100.00 & 100.00 & 100.00 & 99.98 & 100.00 & 100.00 & 100.00 & 100.00 & 100.00 & 100.00 & 100.00 & 100.00 & 100.00 \\
1.0 & 99.69 & 99.23 & 99.27 & 98.59 & 86.14 & 88.16 & 86.51 & 87.54 & 91.51 & 99.03 & 99.61 & 99.88 & 94.56 \\
1.5 & 99.07 & 94.71 & 96.61 & 85.93 & 53.78 & 56.84 & 51.12 & 56.17 & 76.71 & 86.72 & 94.14 & 97.37 & 78.98 \\
2.0 & 93.50 & 87.51 & 90.10 & 63.34 & 29.79 & 28.10 & 31.12 & 31.64 & 54.66 & 66.90 & 84.80 & 91.67 & 62.62 \\
2.5 & 81.41 & 75.83 & 77.70 & 41.42 & 18.18 & 13.46 & 14.03 & 17.64 & 39.95 & 47.97 & 74.35 & 77.93 & 48.17 \\
3.0 & 68.98 & 64.88 & 64.77 & 27.80 & 10.04 & 6.25 & 6.26 & 10.77 & 29.17 & 34.45 & 57.67 & 62.95 & 36.85 \\
3.5 & 59.86 & 53.23 & 53.84 & 18.19 & 5.84 & 2.30 & 2.76 & 6.69 & 21.22 & 24.60 & 44.14 & 50.03 & 28.44 \\
4.0 & 50.51 & 41.65 & 43.25 & 12.37 & 3.47 & 1.25 & 1.02 & 4.23 & 14.46 & 18.58 & 31.17 & 38.89 & 21.65 \\
4.5 & 41.63 & 29.80 & 34.25 & 8.12 & 1.70 & 0.66 & 0.39 & 2.09 & 9.91 & 13.79 & 21.23 & 27.49 & 15.88 \\
5.0 & 31.92 & 22.09 & 25.77 & 5.78 & 0.91 & 0.29 & 0.10 & 0.39 & 7.10 & 9.44 & 14.38 & 19.13 & 11.41 \\
5.5 & 24.06 & 16.72 & 19.73 & 3.92 & 0.55 & 0.08 & 0.00 & 0.13 & 4.66 & 6.69 & 8.63 & 14.23 & 8.26 \\
6.0 & 18.93 & 12.19 & 14.92 & 2.21 & 0.24 & 0.00 & 0.00 & 0.09 & 2.98 & 4.75 & 5.11 & 10.13 & 5.95 \\
6.5 & 15.00 & 9.67 & 10.98 & 1.65 & 0.01 & 0.00 & 0.00 & 0.00 & 2.15 & 3.44 & 3.60 & 7.89 & 4.52 \\
7.0 & 11.21 & 7.61 & 7.66 & 1.08 & 0.00 & 0.00 & 0.00 & 0.00 & 1.71 & 2.35 & 2.39 & 5.88 & 3.31 \\
7.5 & 9.22 & 5.95 & 5.71 & 0.73 & 0.00 & 0.00 & 0.00 & 0.00 & 1.50 & 1.75 & 1.44 & 4.78 & 2.58 \\
8.0 & 7.55 & 4.93 & 4.32 & 0.46 & 0.00 & 0.00 & 0.00 & 0.00 & 1.27 & 1.36 & 0.96 & 3.88 & 2.05 \\
8.5 & 6.17 & 4.12 & 3.00 & 0.22 & 0.00 & 0.00 & 0.00 & 0.00 & 0.97 & 1.11 & 0.77 & 3.03 & 1.61 \\
$9.0^{*}$ & 4.48 & 3.11 & 2.02 & 0.14 & 0.00 & 0.00 & 0.00 & 0.00 & 0.51 & 0.90 & 0.52 & 2.23 & 1.15 \\
13.5 & 0.00 & 0.00 & 0.00 & 0.00 & 0.00 & 0.00 & 0.00 & 0.00 & 0.00 & 0.00 & 0.00 & 0.00 & 0.00 \\
\hline
\end{tabular}

Figure B.3: Distribution Spectral peak periods around the british Isles: Figure 2 in [41] 
Lines of Constant Steepness

Total Samples $\mathbf{7 8 8 4 3}$

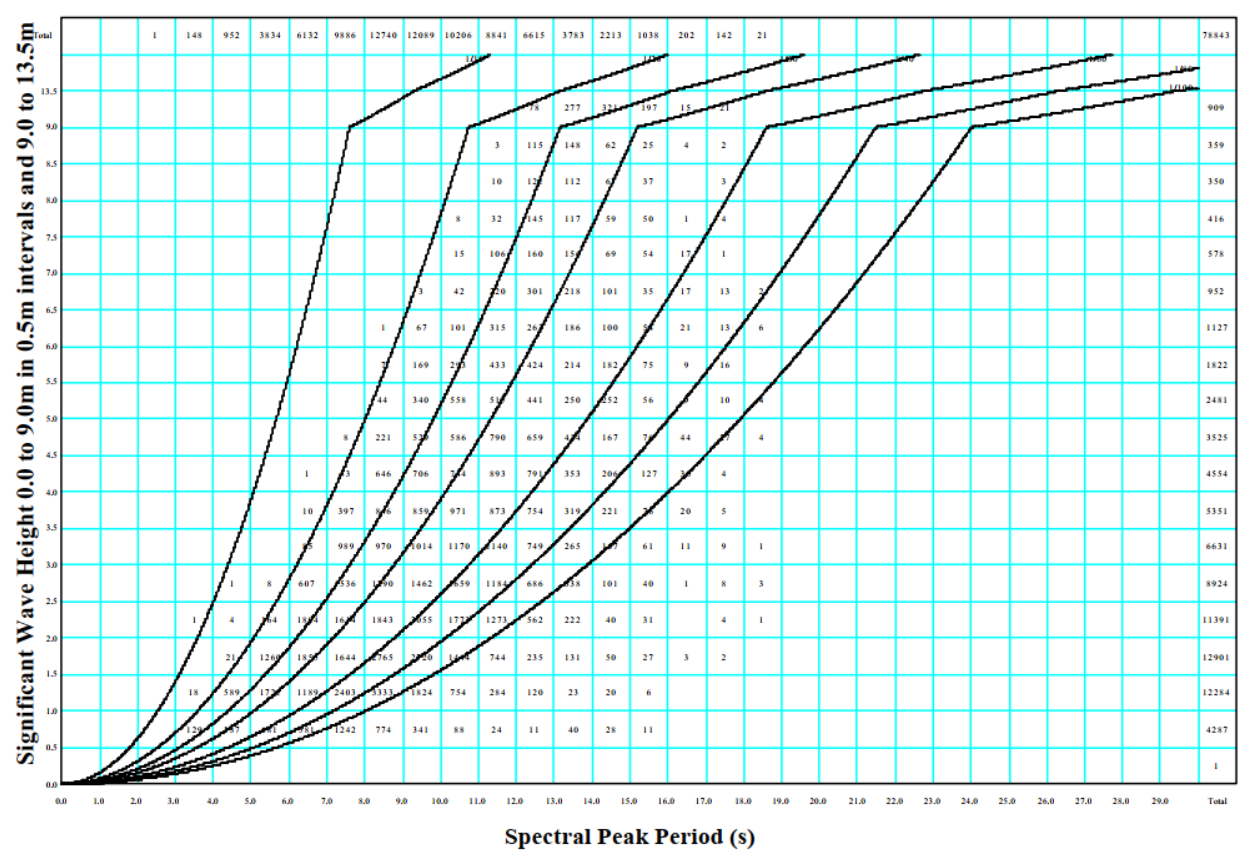

Figure B.4: Distribution Spectral peak periods around the british Isles: Figure 2 in [41] 



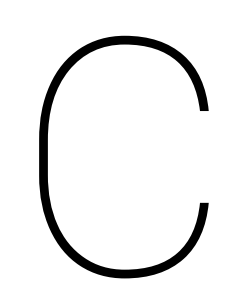

\section{Results variable turbine diameter}

\section{C.1. Results weir level 0.5 meter}

C.1.1. Turbine diameter 1.5 meter

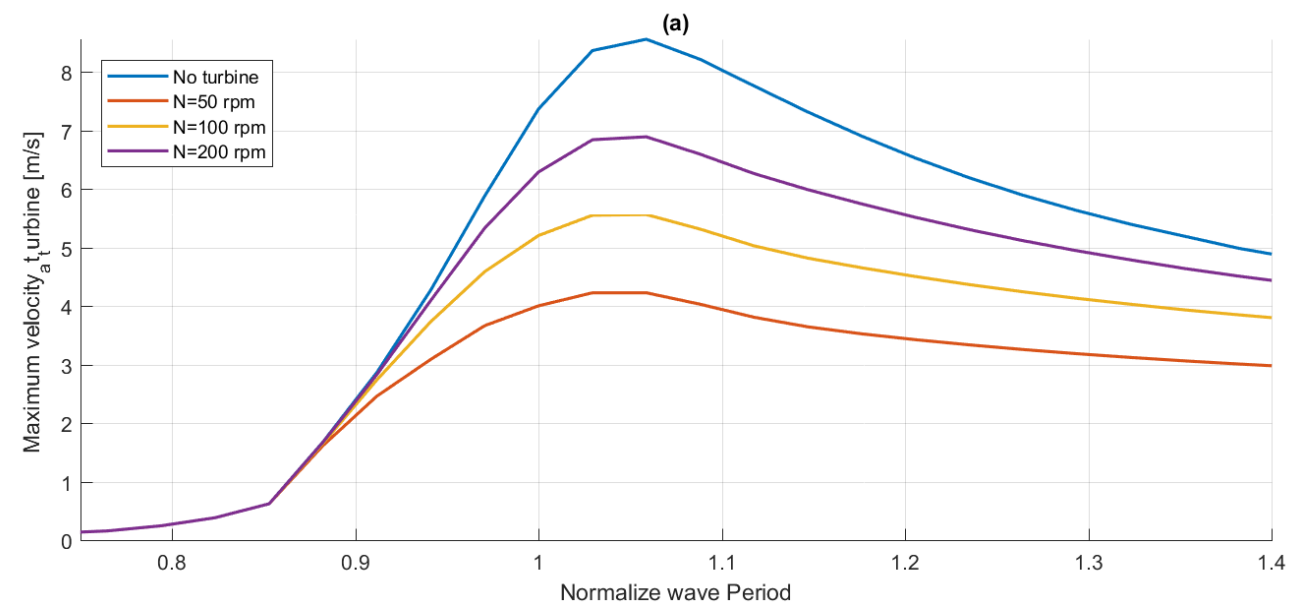

Figure C.1: Maximum velocity at turbine for $D_{t}=1.5 \mathrm{~m}$

(a)

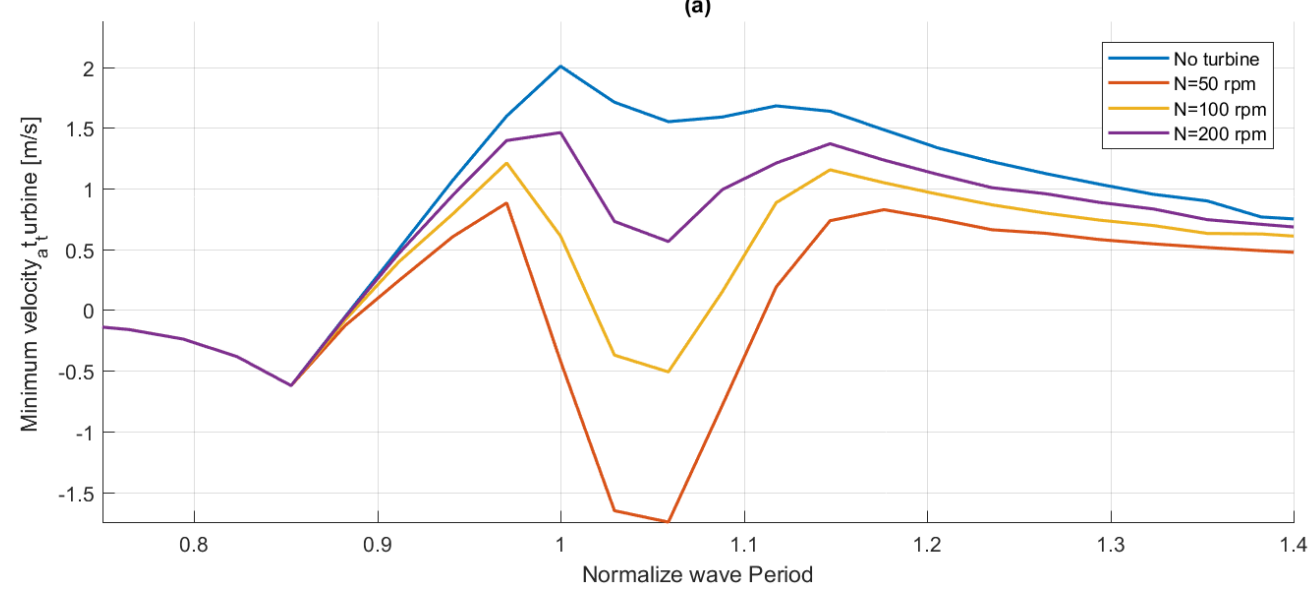

Figure C.2: Minimum velocity at turbine for $D_{t}=1.5 \mathrm{~m}$ 
(a)

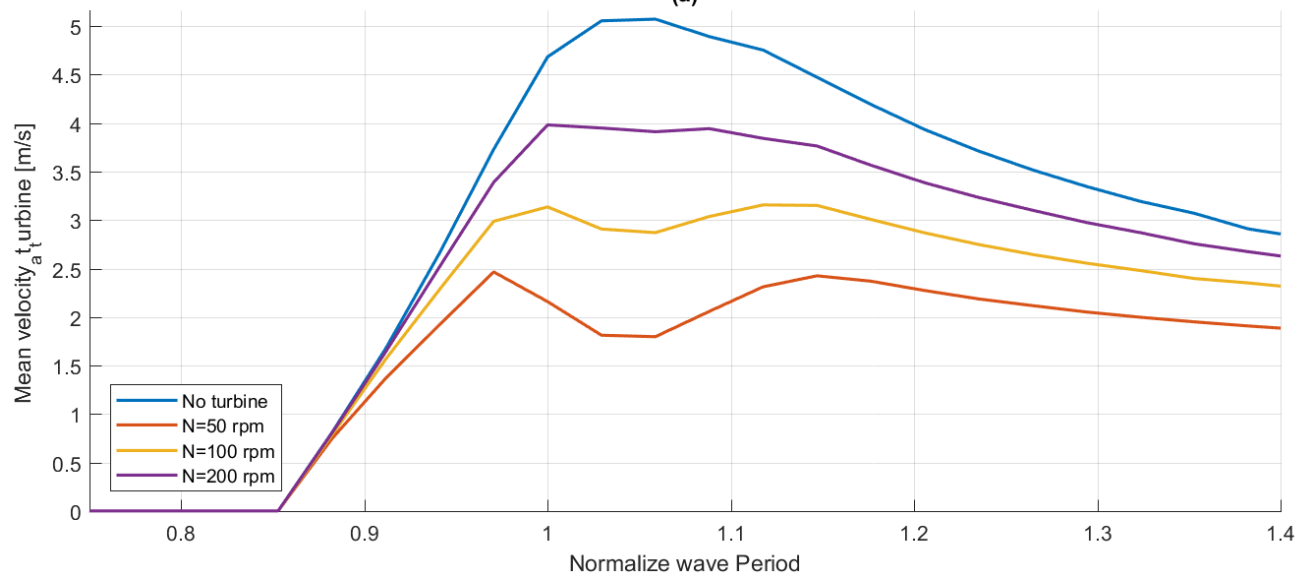

Figure C.3: Mean velocity at turbine for $D_{t}=1.5 \mathrm{~m}$

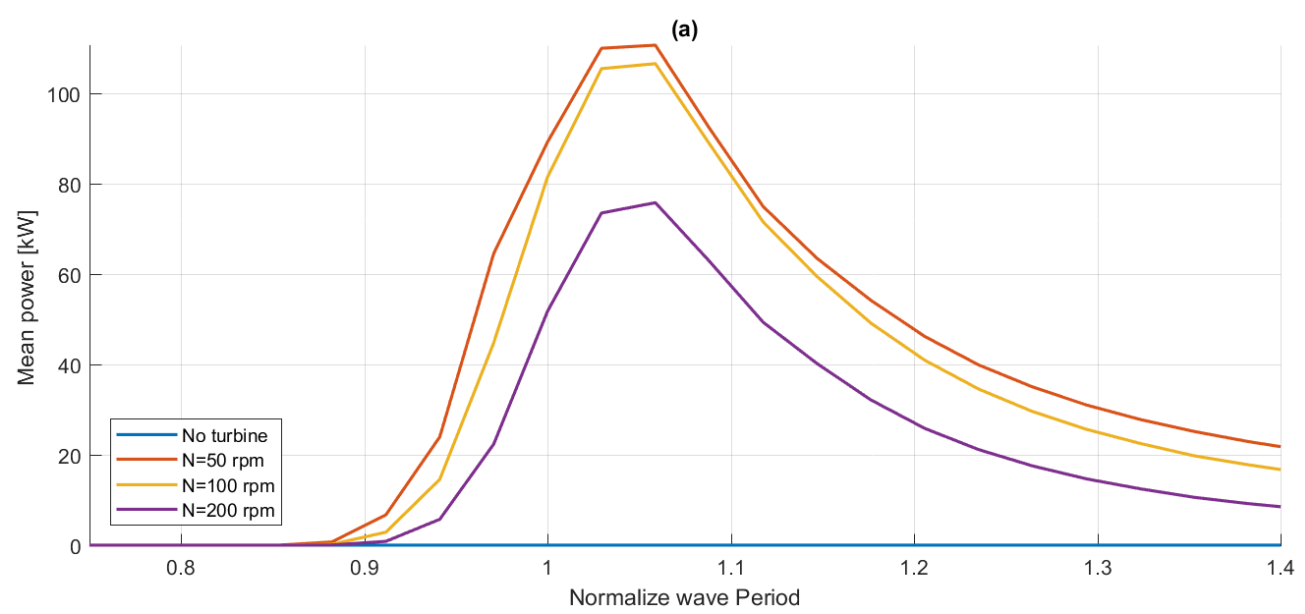

Figure C.4: Mean power for different rotation speeds for $D_{t}=1.5 \mathrm{~m}$

\section{C.1.2. Turbine diameter of 1.75 meter}

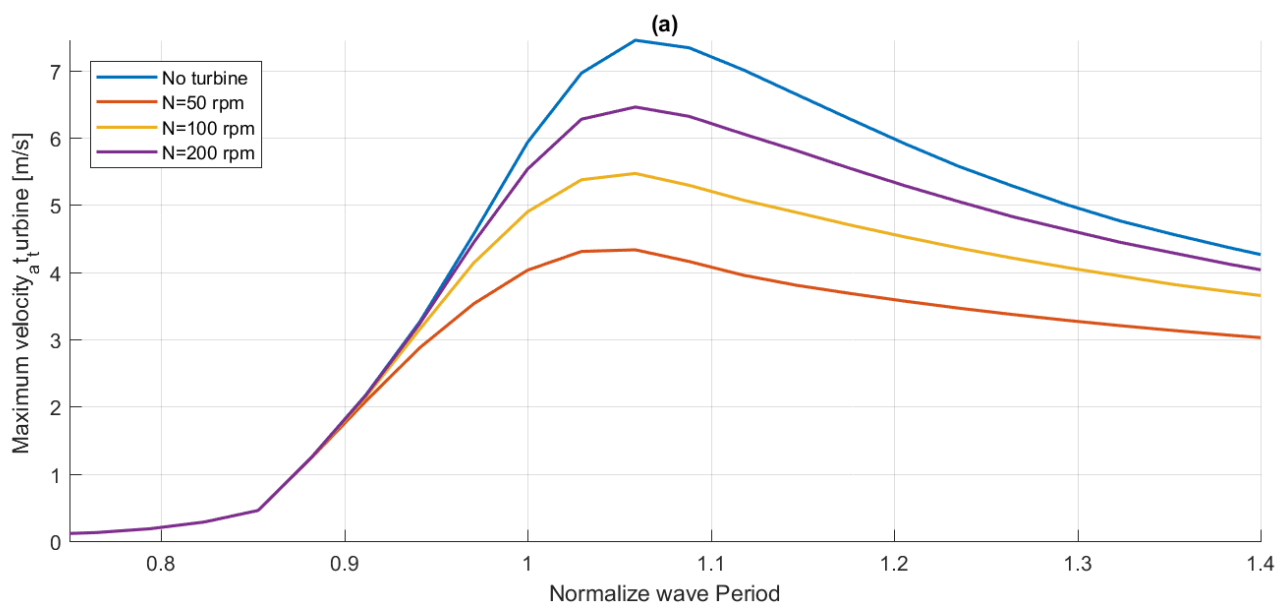

Figure C.5: Maximum velocity at turbine for $D_{t}=1.75 \mathrm{~m}$ 


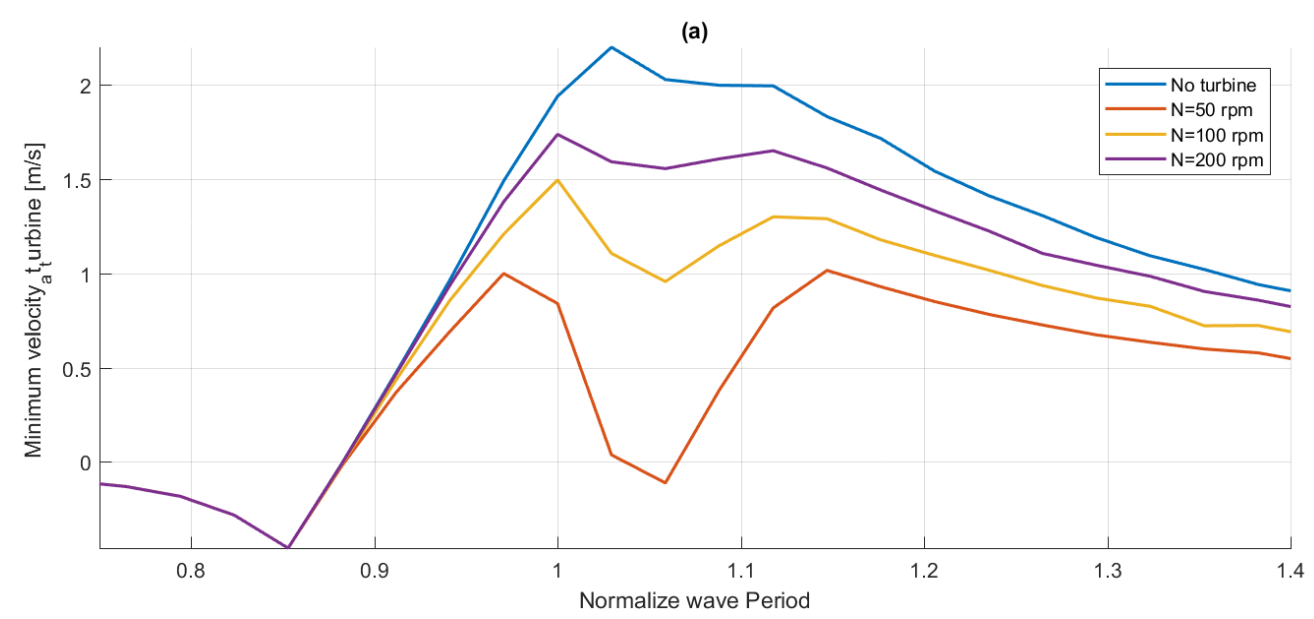

Figure C.6: Minimum velocity at turbine for $D_{t}=1.75 \mathrm{~m}$

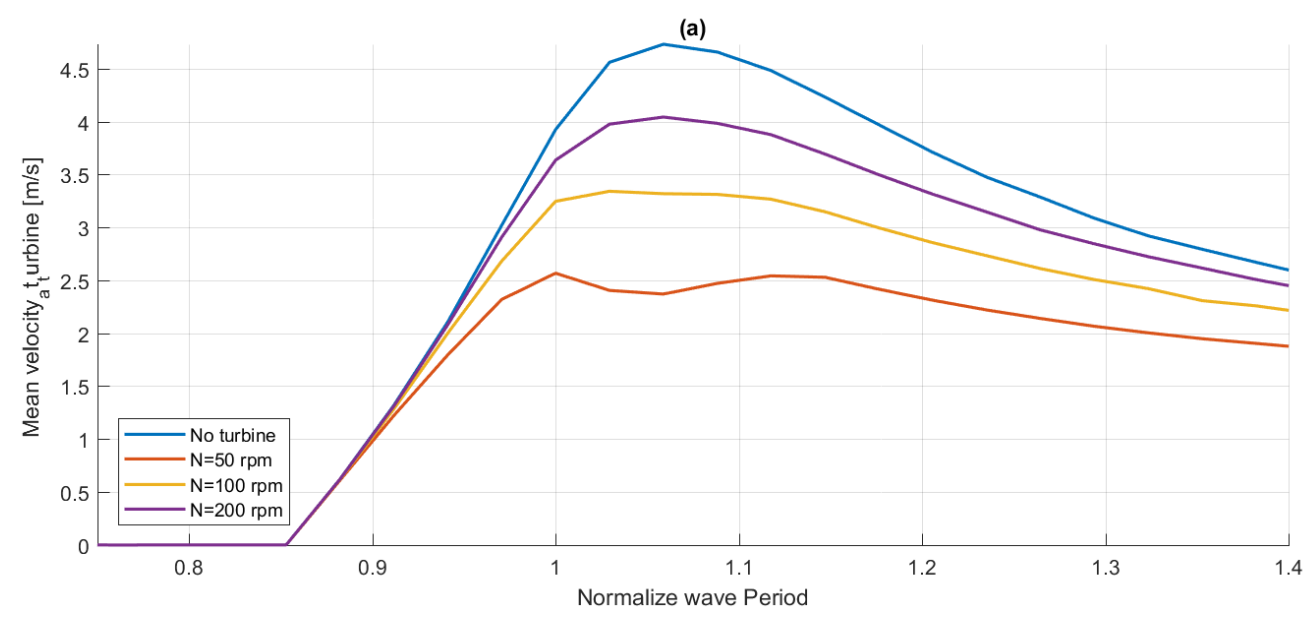

Figure C.7: Mean velocity at turbine for $D_{t}=1.75 \mathrm{~m}$

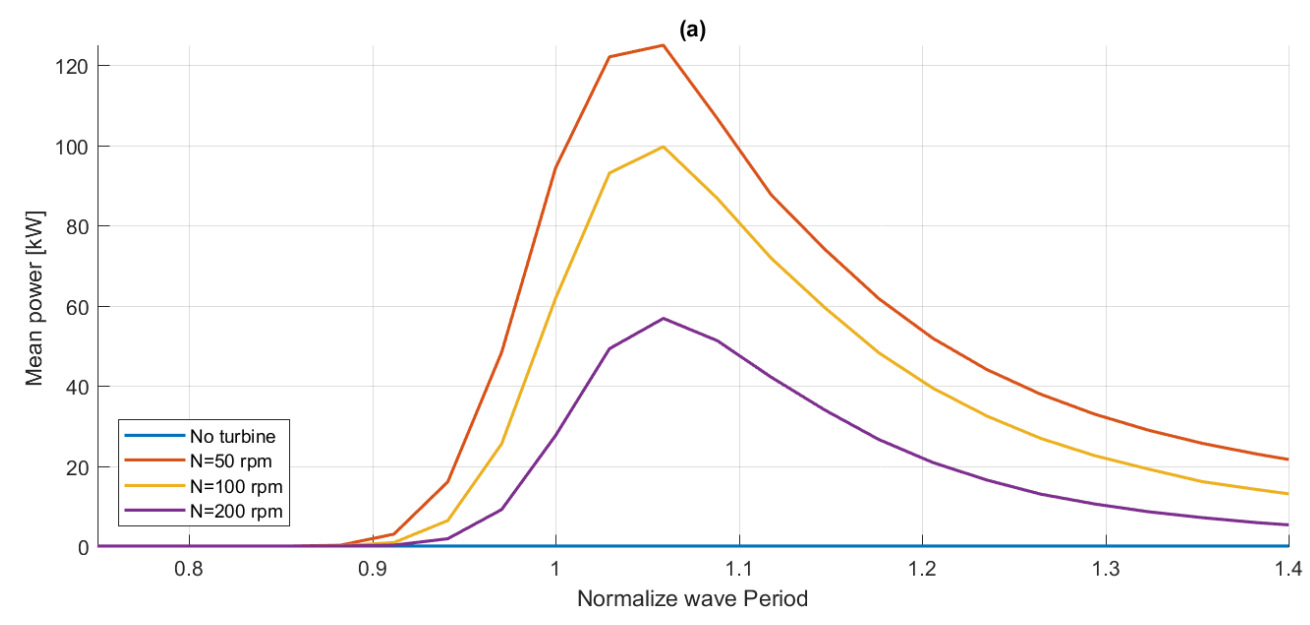

Figure C.8: Mean power for different rotation speeds for $D_{t}=1.75 \mathrm{~m}$ 


\section{C.1.3. Turbine diameter of 2 meter}

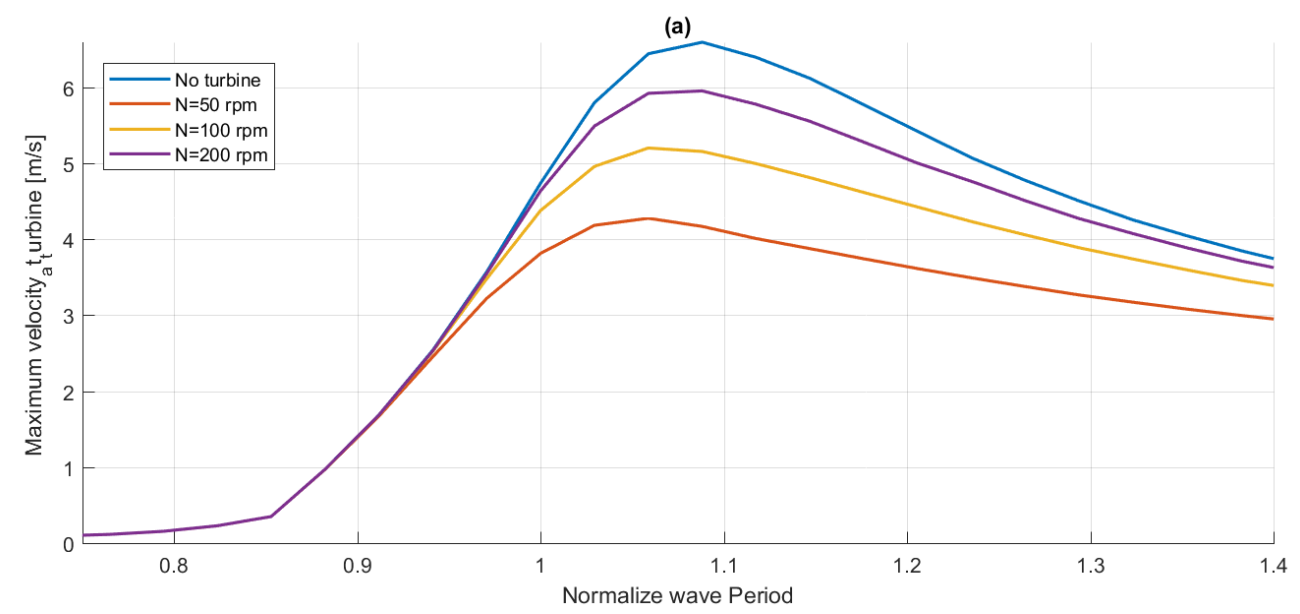

Figure C.9: Maximum velocity at turbine for $D_{t}=2 \mathrm{~m}$

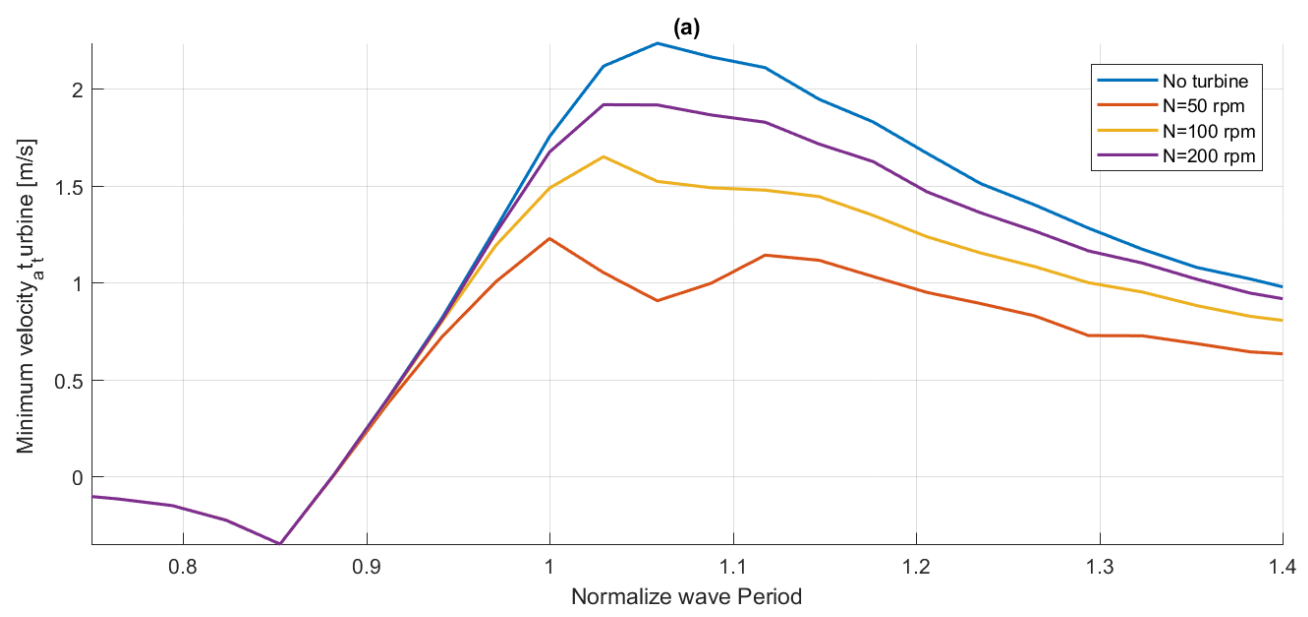

Figure C.10: Minimum velocity at turbine for $D_{t}=2 \mathrm{~m}$

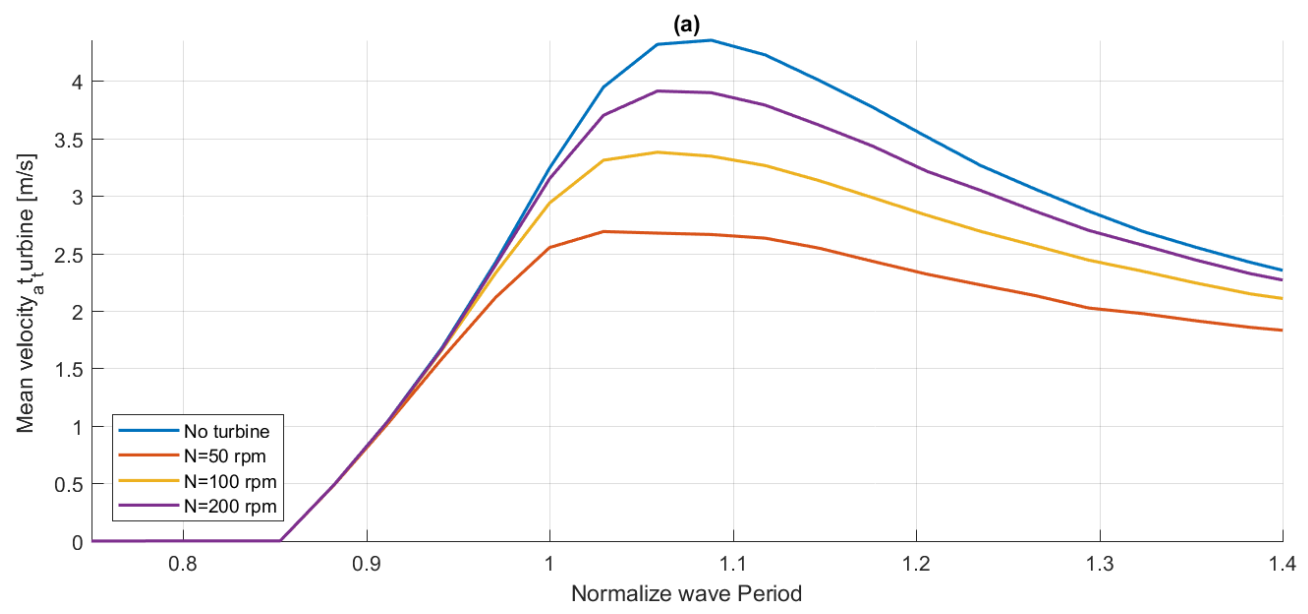

Figure C.11: Mean velocity at turbine for $D_{t}=2 \mathrm{~m}$ 


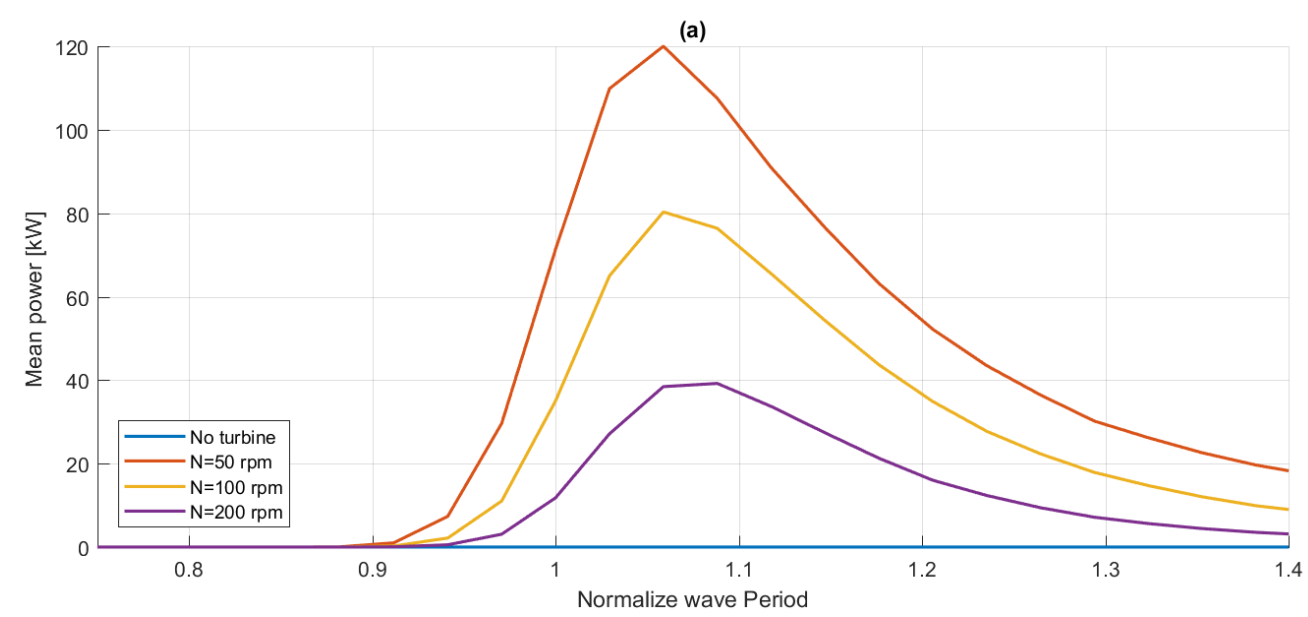

Figure C.12: Mean power for different rotation speeds for $D_{t}=2 \mathrm{~m}$

\section{C.1.4. Turbine diameter of 2.25 meter}

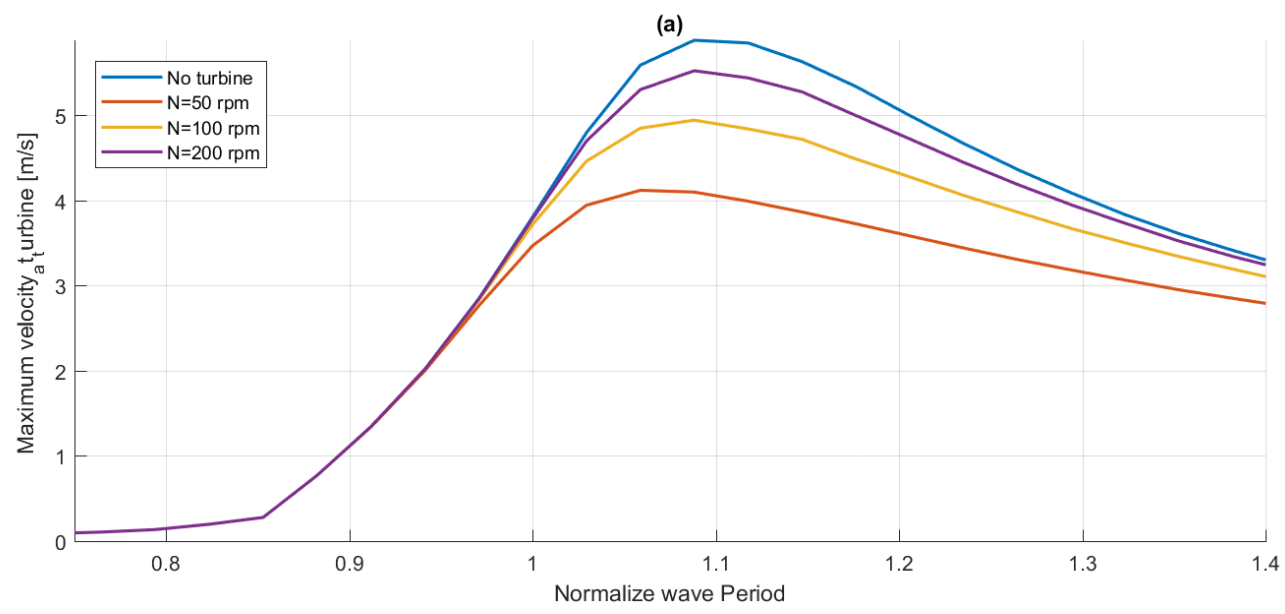

Figure C.13: Maximum velocity at turbine for $D_{t}=2.25 \mathrm{~m}$

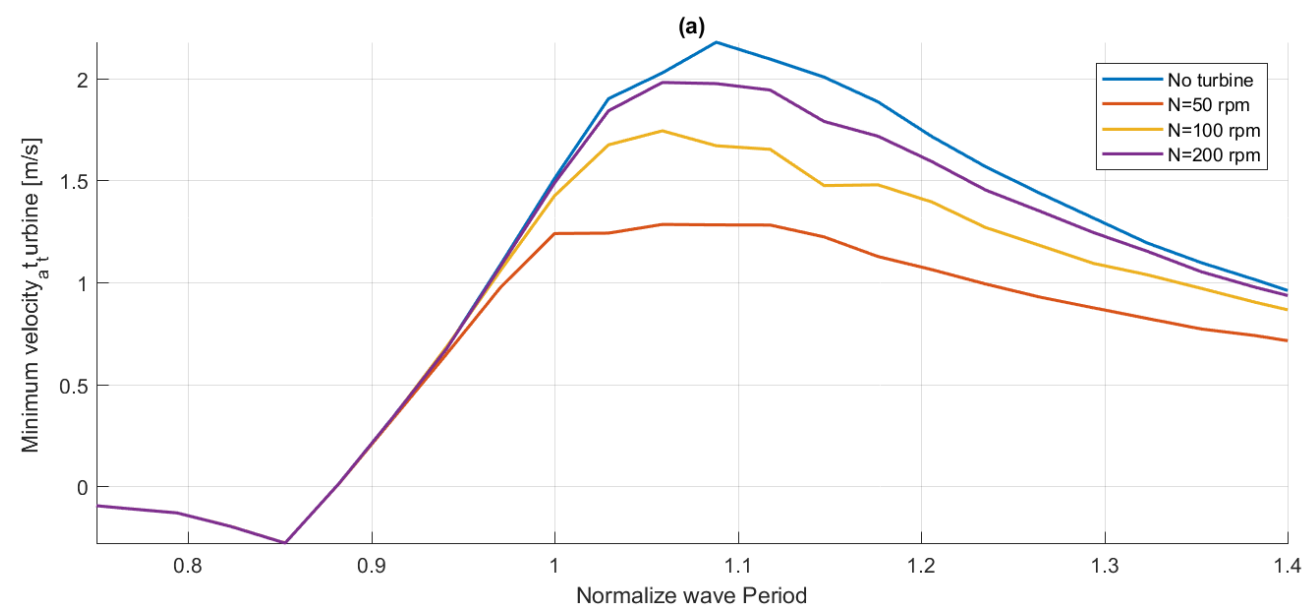

Figure C.14: Minimum velocity at turbine for $D_{t}=2.25 \mathrm{~m}$ 


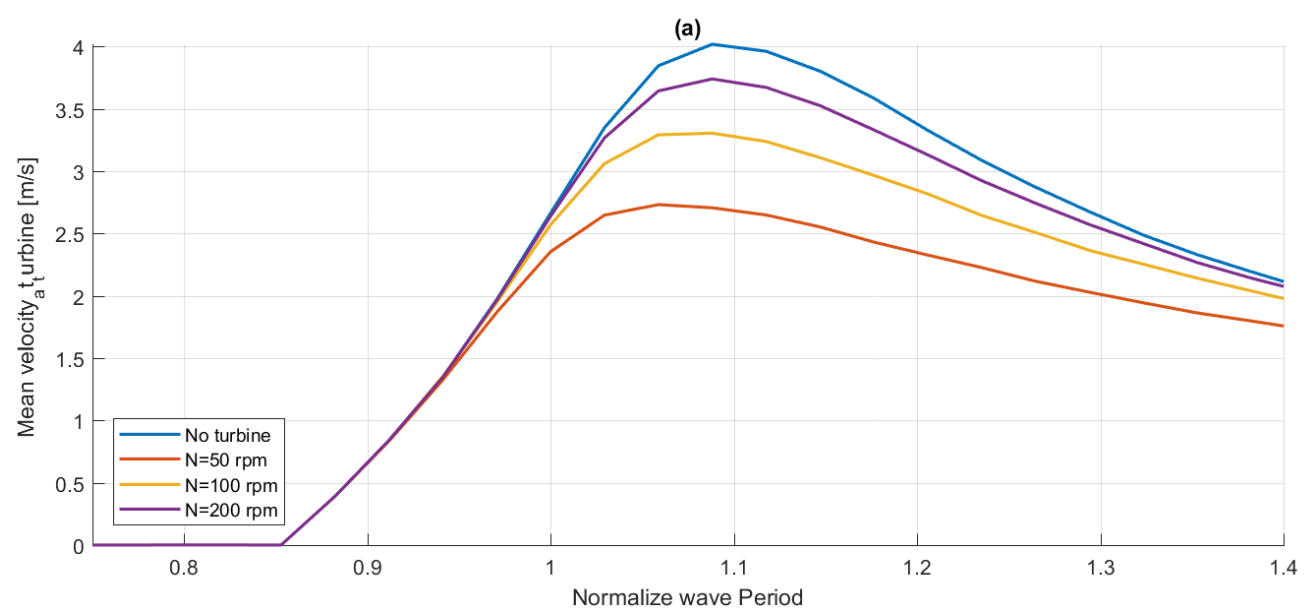

Figure C.15: Mean velocity at turbine for $D_{t}=2.25 \mathrm{~m}$

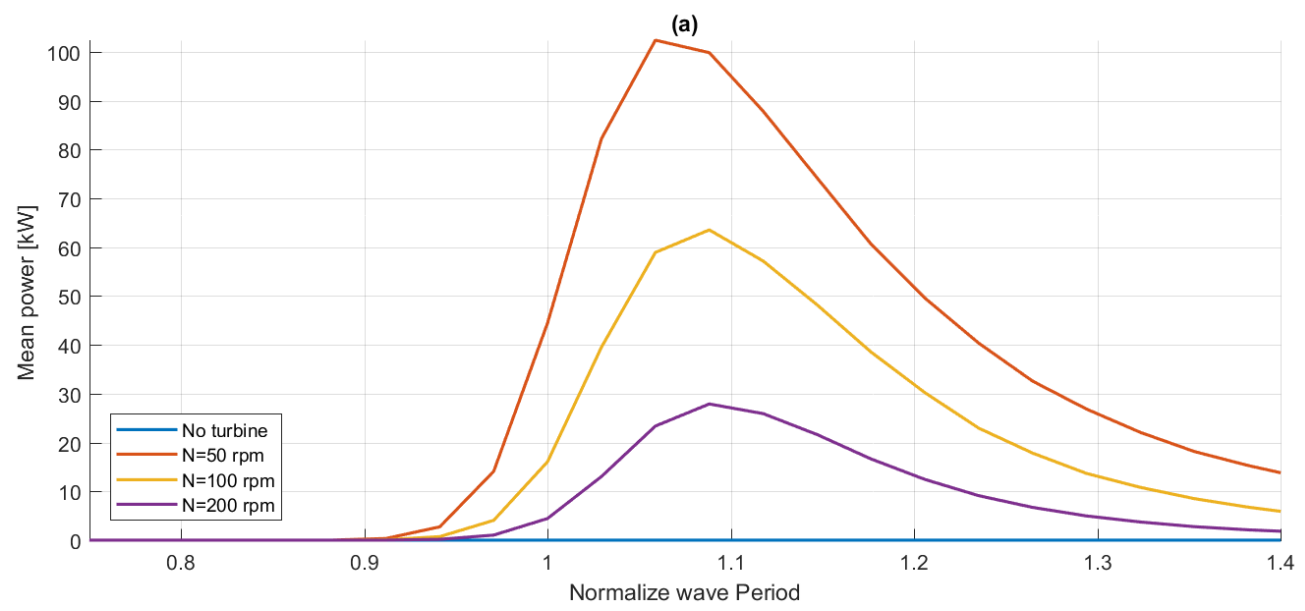

Figure C.16: Mean power for different rotation speeds for $D_{t}=2.25 \mathrm{~m}$

\section{C.1.5. Turbine diameter of 2.5 meter}

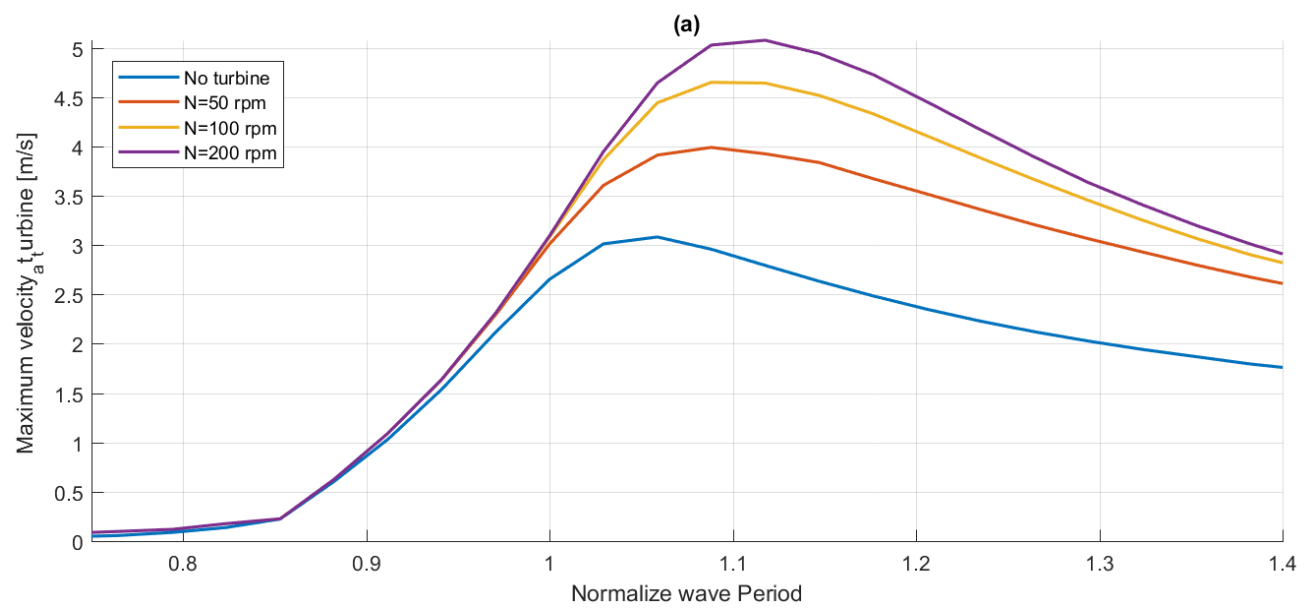

Figure C.17: Maximum velocity at turbine for $D_{t}=2.5 \mathrm{~m}$ 


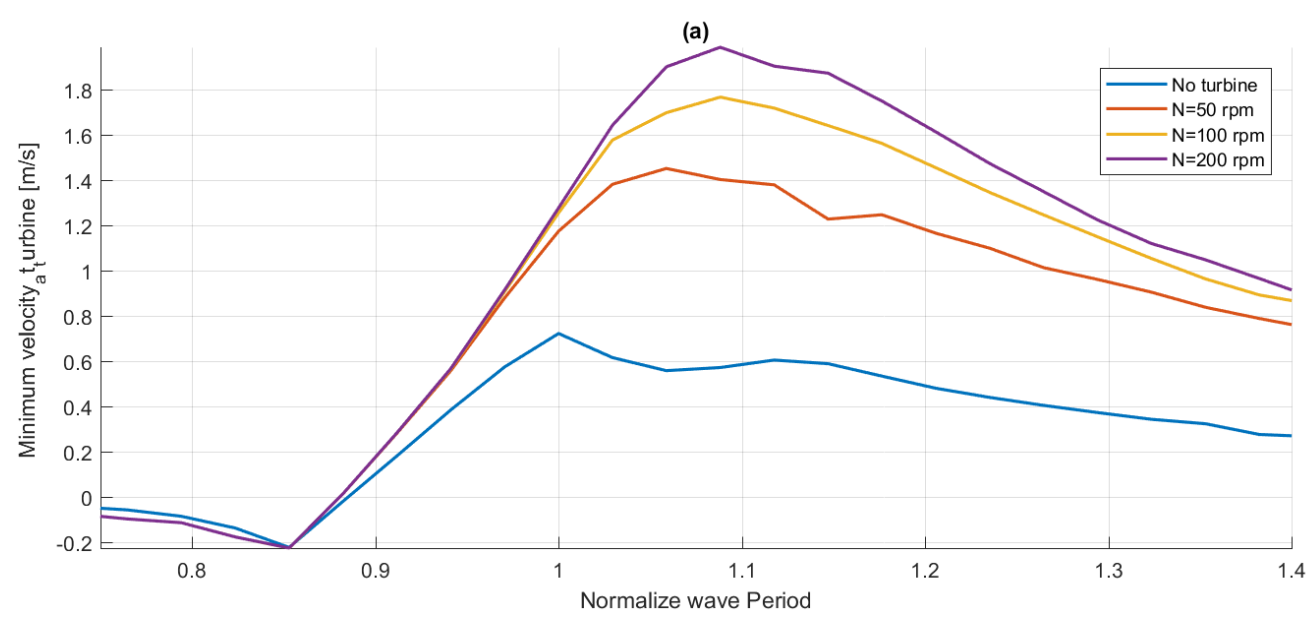

Figure C.18: Minimum velocity at turbine for $D_{t}=2.5 \mathrm{~m}$

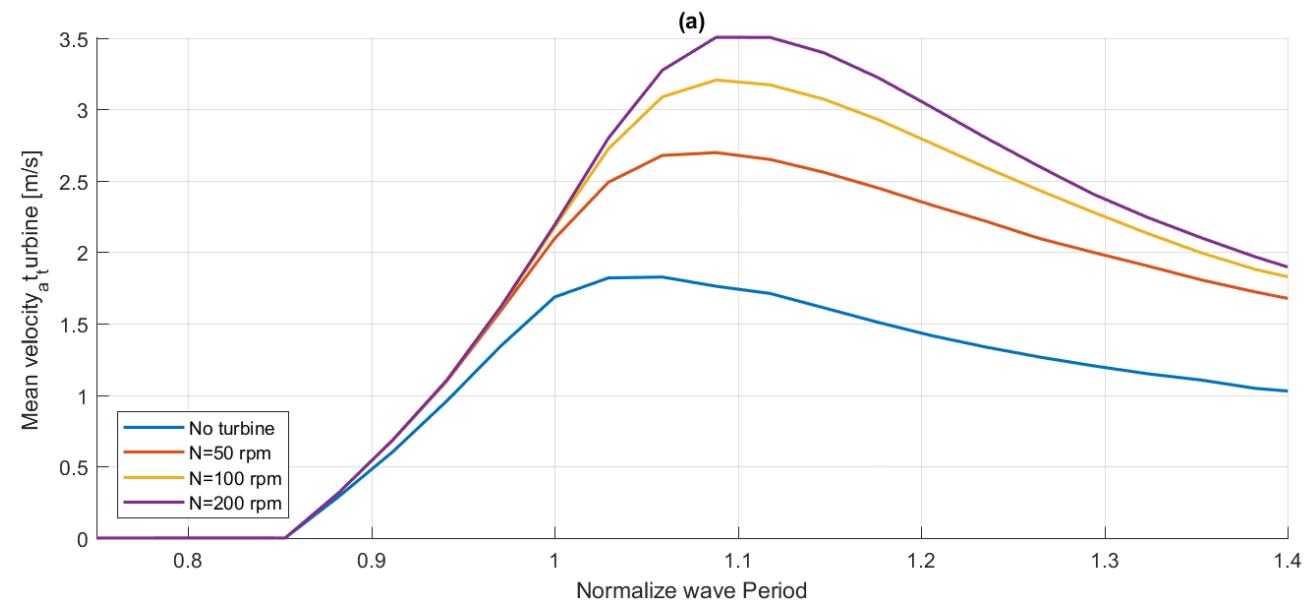

Figure C.19: Mean velocity at turbine for $D_{t}=2.5 \mathrm{~m}$

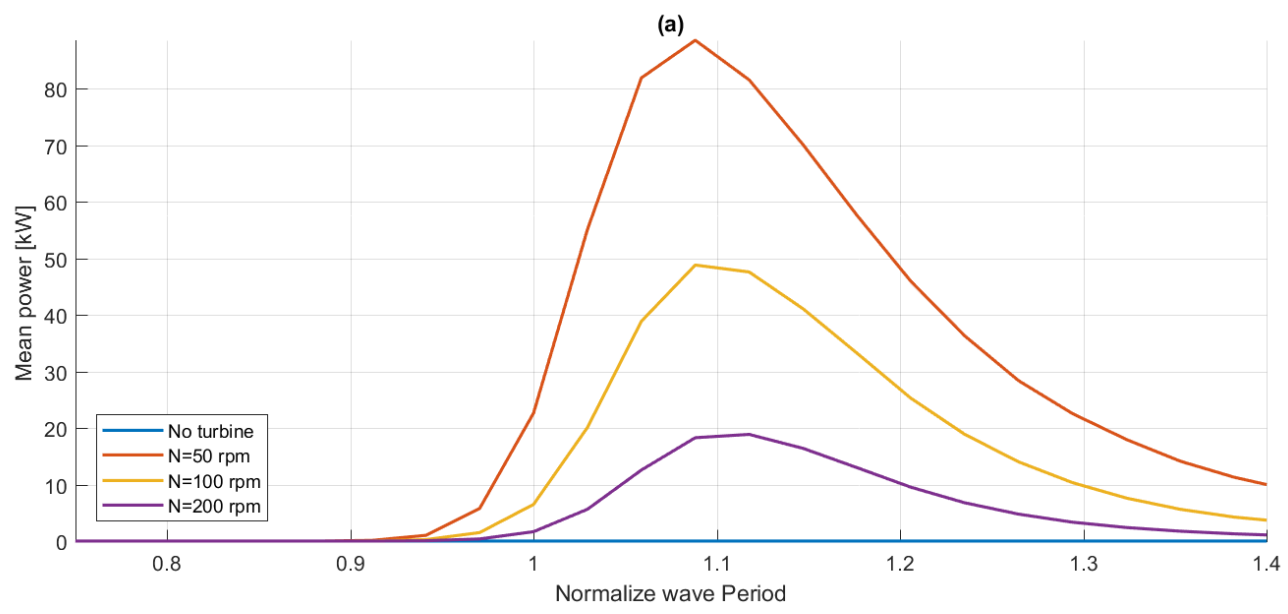

Figure C.20: Mean power for different rotation speeds for $D_{t}=2.5 \mathrm{~m}$ 


\section{C.2. Results weir level 0.7 meter}

\section{C.2.1. Turbine diameter of 2 meter}

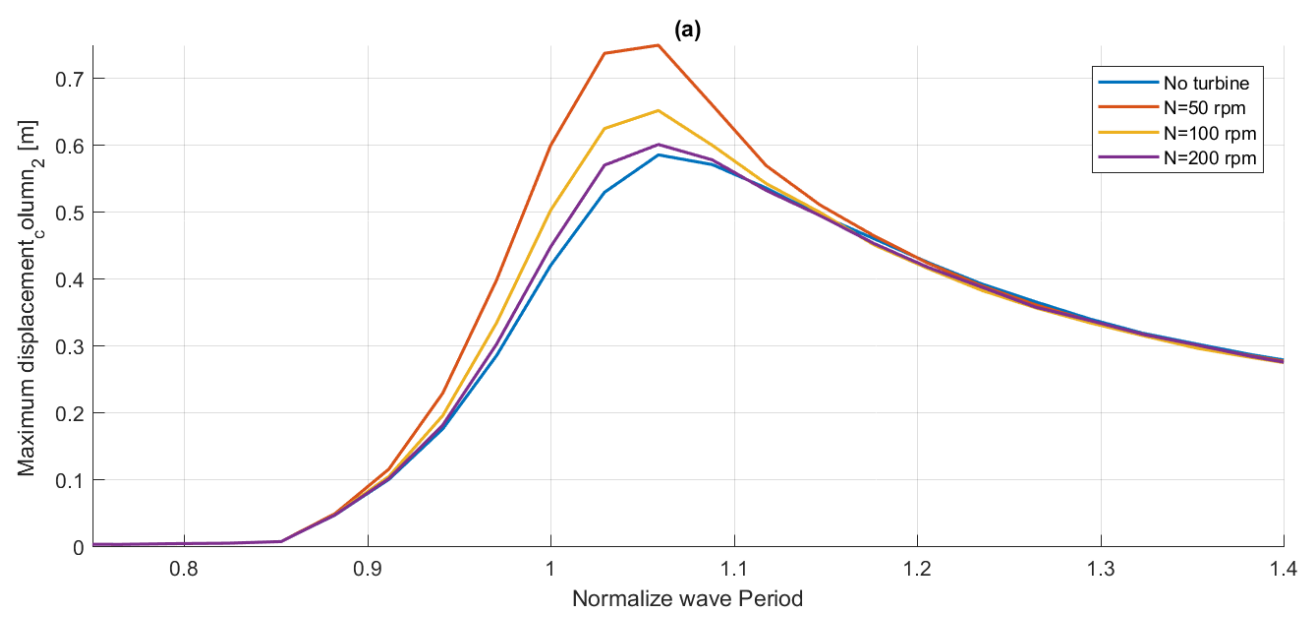

Figure C.21: Maximum velocity at turbine for $D_{t}=2 \mathrm{~m}$ and $z_{w}=0.7 \mathrm{~m}$

(a)

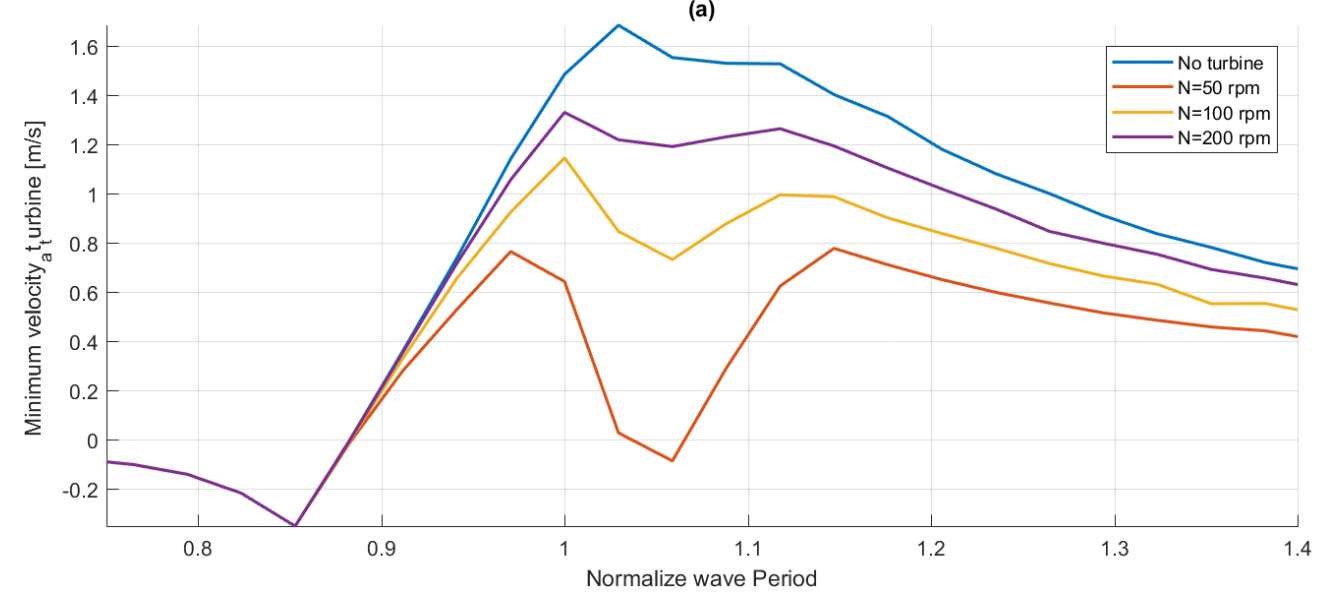

Figure C.22: Minimum velocity at turbine for $D_{t}=2 \mathrm{~m}$ and $z_{w}=0.7 \mathrm{~m}$ 


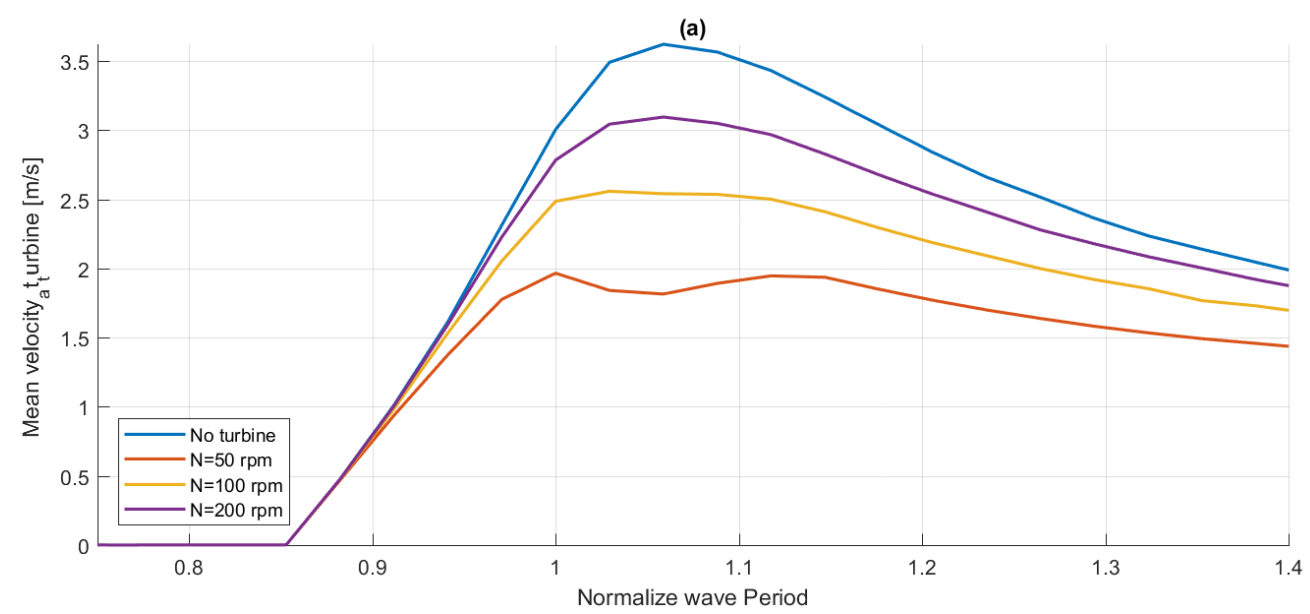

Figure C.23: Mean velocity at turbine for $D_{t}=2 \mathrm{~m}$ and $z_{w}=0.7 \mathrm{~m}$

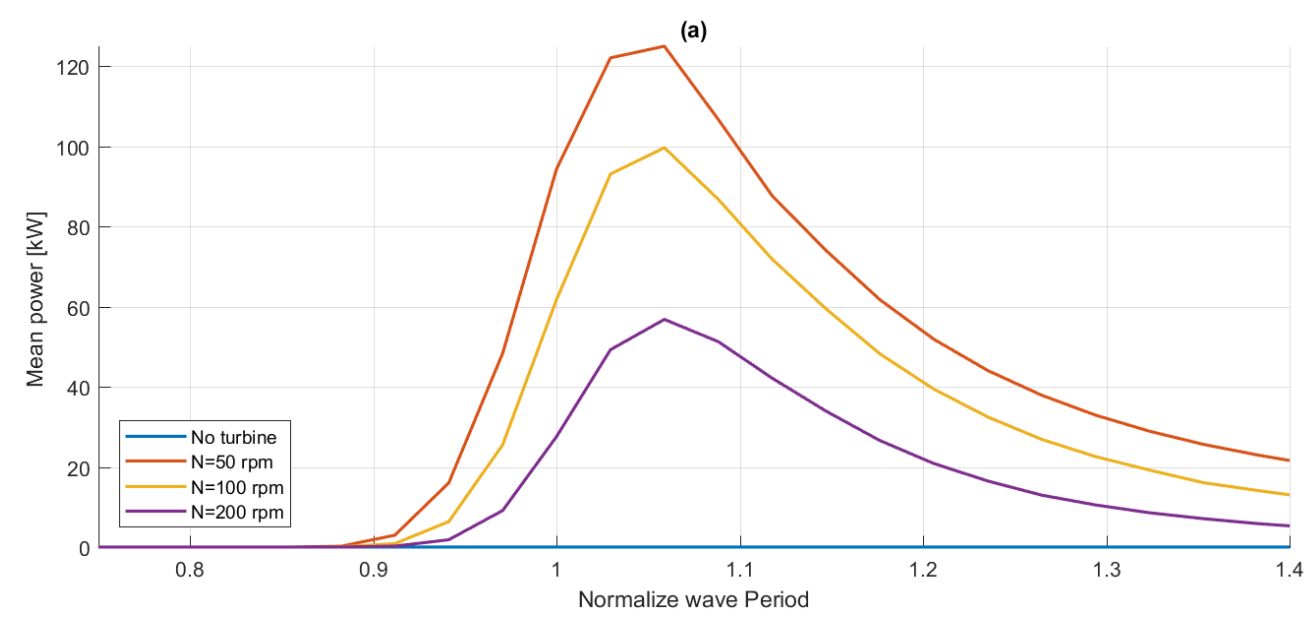

Figure C.24: Mean power for different rotation speeds for $D_{t}=2 \mathrm{~m}$ and $z_{w}=0.7 \mathrm{~m}$ 



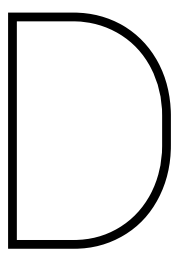

\section{Glasgow scale tests Neptune wave energy converter}

\section{D.1. Glasgow scale tests}

In figure D. 1 and D. 2 the settings of the scale test performed in glasgow 2008 are presented. 


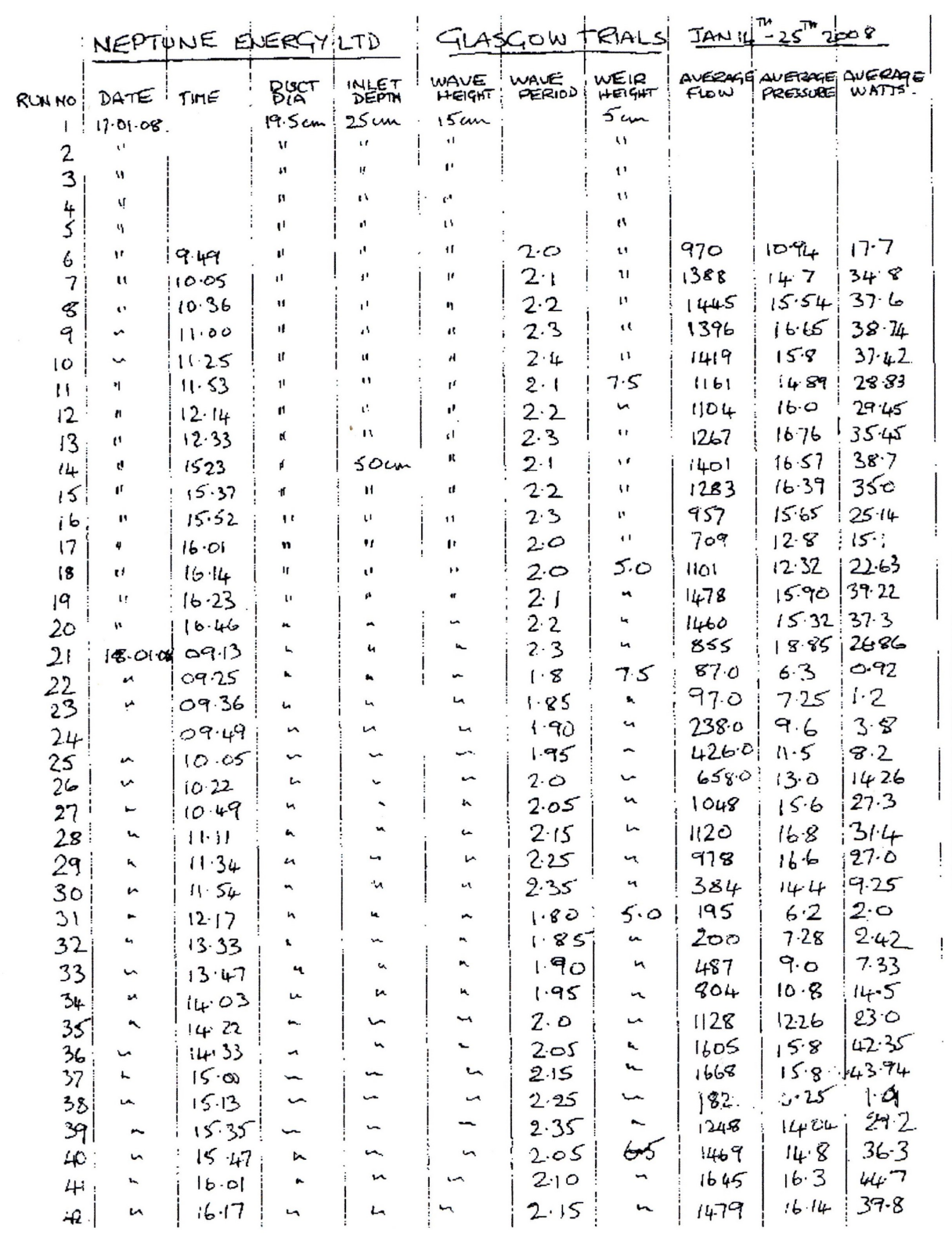

Figure D.1: Hand written notes settings and average results Glasgow testing: part 1 


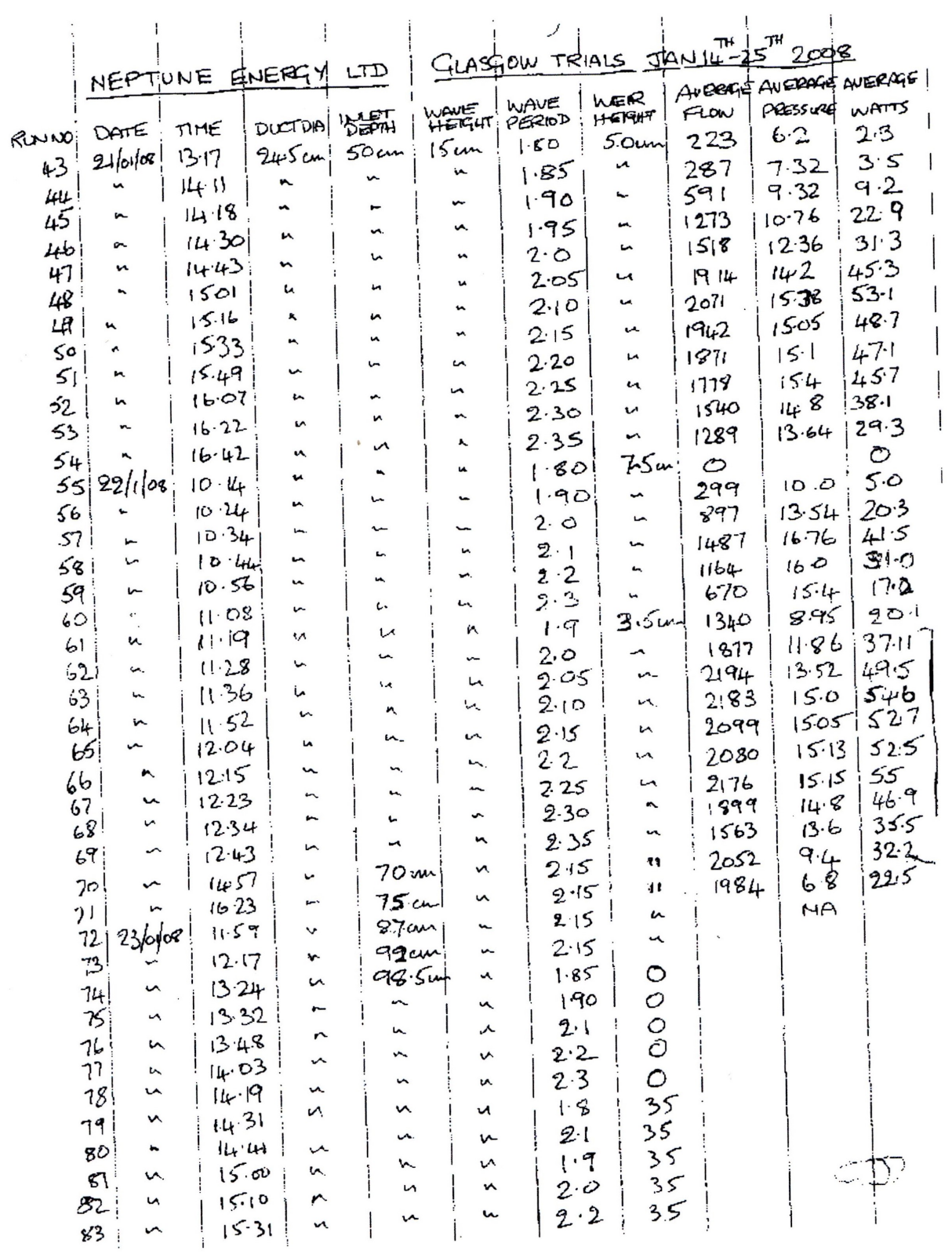

Figure D.2: Hand written notes settings and average results Glasgow testing: part 2 


\section{D.2. Plymouth test}

In this section the result section of the experimental report of the Plymouth test is given. 
All monochromatic wave tests were run for a period of 128 seconds with a 2 second paddle ramp time at the beginning and end of each run. This time was considered adequate to allow the incident and reflected waves in the basin to stabilise giving a 64 s operating window for data analysis. Settling time between each run varied depending on the previous incident wave conditions, and enough time was given after each test to allow the internal water level of the device to become still.

The three irregular wave tests are repeats of a single long-crested irregular run based on a JONSWAP wave energy spectrum, defined by:

- $\quad$ Peak period $(\mathrm{Tp})=2.4 \mathrm{~s}$

- Significant wave height $(\mathrm{Hs})=0.25 \mathrm{~m}$

- Gamma = 3.3

This spectrum was chosen as a realistic design simulation based around the optimum operating frequency scaled to $1: 20^{\text {th }}$. However, such a spectrum is not expected to accurately describe site specific conditions without further assessment of any specific proposed deployment site.

Irregular wave tests were run for a repeat period of 402 seconds; this corresponds to a prototype scale time period of 30 minutes. This time series is considered an appropriate length to obtain accurate harmonic components of a sea-state (http://www.emec.org.uk/tanktesting-of-wave-energy-conversion-systems/) and will provide a good representation of device operational performance.

\section{RESULTS}

\subsection{Conditioning}

All calibrated pressure and velocity records for each run have previously been provided to LW for further analysis. A $10 \mathrm{~Hz}$ low-pass filter has been used to process the pressure signals.

Summary values for each pressure sensor are supplied in a separate spreadsheet via email alongside this technical note. The raw pressure data for each test was transformed to a level so that the mean still water value at the beginning of each test is equal to zero. The pressure recordings in the air chamber oscillate around this datum. Once flow is introduced in the outlet pipe, however, the mean value of the static pressure recording reduces. Any negative pressures are negative relative to the initial still water pressure experienced within the Neptune device.

Appendix A summarises each test in terms of velocity and power output. The values represent a mean and maximum value within the optimum $64 \mathrm{~s}$ window. The mean power values, therefore, are derived from the time-series of velocity measurements. In addition, a mean of all the peak values within that window is presented. For the irregular wave tests the $402 \mathrm{~s}$ window has been applied. 
An additional column for a quality check of each test is given in Appendix A. This provides a visual check of the amount of stalling seen when comparing the data time-series with the corresponding video from inside the outlet pipe. This is merely a guide to the reliability of the presented data. A key to the QC values given in Appendix $A$ is presented below.

$\begin{aligned} \text { Key } & \\ 0= & \text { Zero flow } \\ 1= & \text { Unidirectional flow } \\ 2= & \text { Unidirectional flow } \\ 3= & \text { Unidirectional flow } \\ 4= & \text { Bidirectional flow } \\ 5= & \text { Zero net flow }\end{aligned}$

\subsection{Power calculations}

In a circular pipe of radius $R$ it is assumed (Equation 1) that velocity across the radius of the pipe, $u_{r}$, assumes a parabolic profile from $u_{r}=0$ at the boundary (where $r=R$ ) to $u_{r}=u_{\max }$ at the centre of the pipe (where $r=0$ ). With the water velocity measured at the centre of the pipe being $u_{\max }$ the average instantaneous velocity $(v)$ throughout the cross sectional area of the pipe is calculated by Equation 2. This velocity time series is then used to calculate the summary statistics.

Equation 1

$$
u_{r}=u_{\max }\left[1-\left(\frac{r}{R}\right)^{2}\right] \quad\left(m s^{-1}\right)
$$

Equation 2

$$
u_{\text {avg }}=\frac{u_{\max }}{2} \quad\left(m s^{-1}\right)
$$

Power in the flow $(P)$ can be calculated directly from volume flow rate $(Q)$ and dynamic pressure $\left(p_{d}\right)$ (Equations $\left.3-5\right)$, where $\rho$ is the density of the water $\left(1000 \mathrm{~kg} \mathrm{~m}^{-3}\right)$ and $A$ is the cross-sectional area of the pipe.

Equation 3

$$
p_{d}=1 / 2 \rho v^{2}
$$

Equation 4

$$
Q=v A \quad\left(m^{2} s^{-1}\right)
$$

Equation 5

$$
P=p_{d} Q
$$

This calculation is based on a number of assumptions:

- the flow is irrotational,

- the flow is laminar, and pipe end-effects are eliminated, 
- the fluid is incompressible, and

- 'no-slip' boundary condition exists.

Figures 1 - 7 show the frequency response of the device with respect to the mean power calculated in the outlet pipe flow for the monochromatic wave tests. Note that multiple runs of the same forcing conditions are shown.

The irregular wave tests, with a weir height of $65 \mathrm{~mm}$ at a water depth of $1.85 \mathrm{~m}$, based on the scaled JONSWAP spectrum routinely yielded a mean power of approximately $0.2 \mathrm{~W}$, with peak power found to be of the order $5 \mathrm{~W}$.

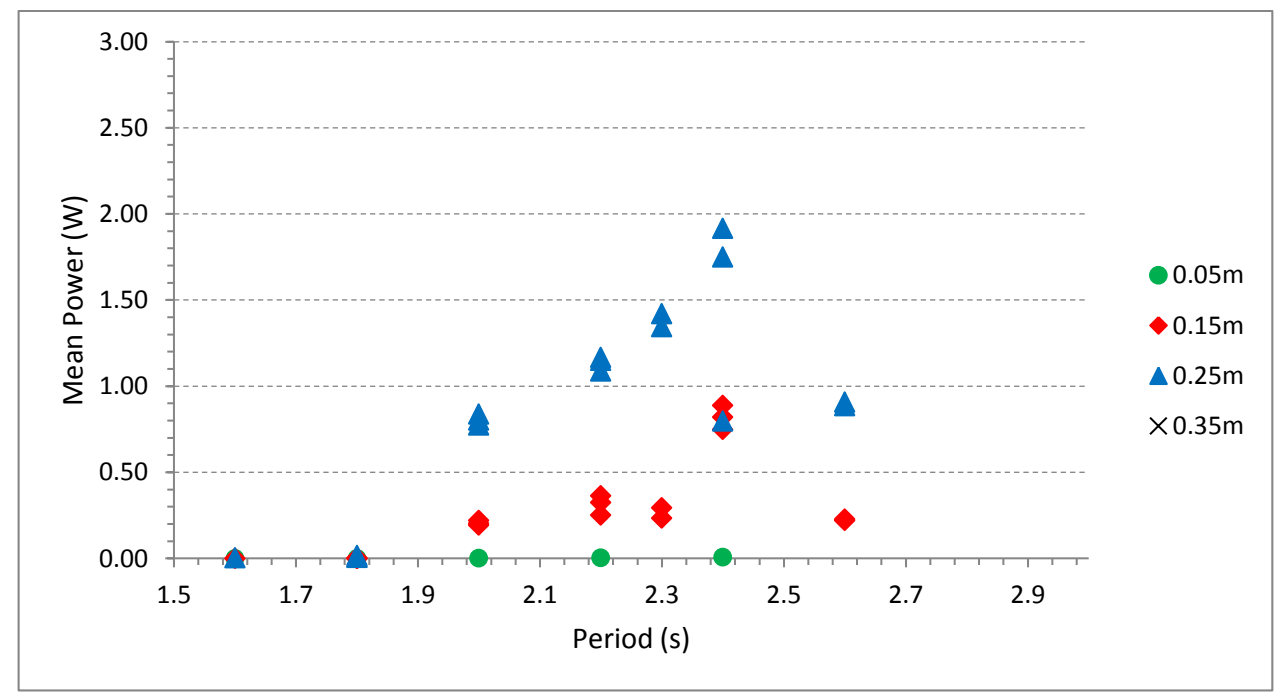

Figure 1: Mean power against wave frequency calculated from model tests with wave-heights of $0.05 \mathrm{~m}, 0.15 \mathrm{~m}$ and $0.25 \mathrm{~m}$ with water depth $=1.85 \mathrm{~m}$ and weir height $=35 \mathrm{~mm}$ 


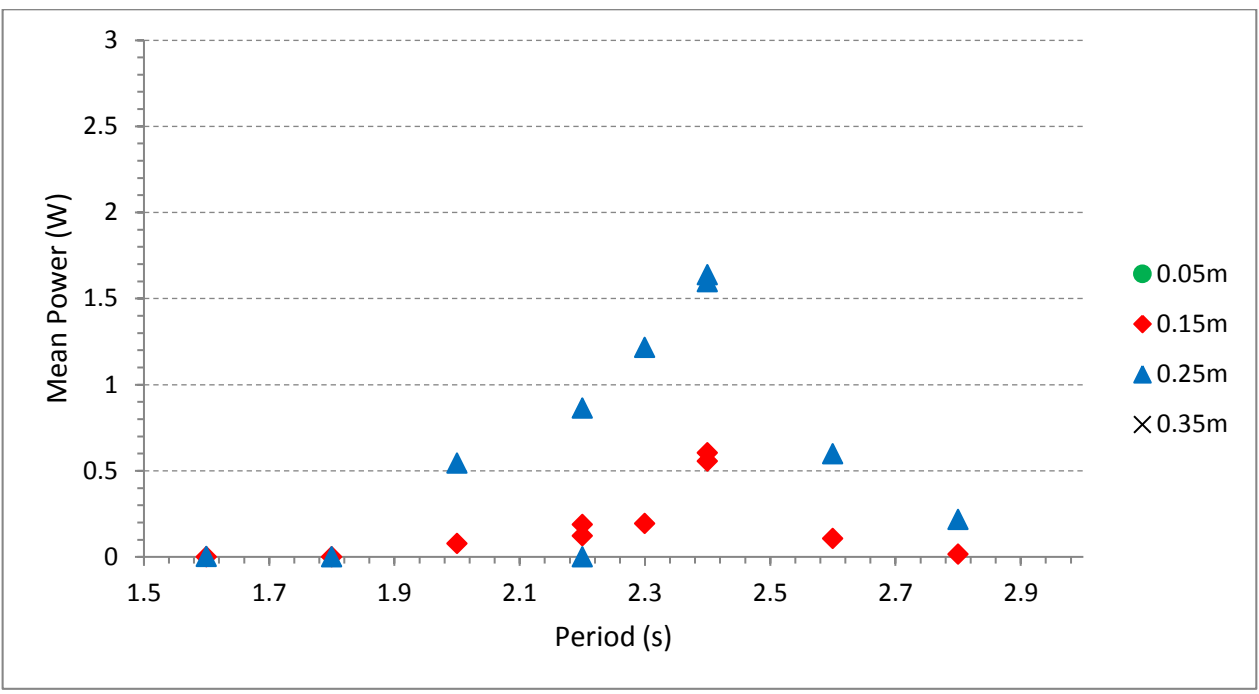

Figure 2: Mean power against wave frequency calculated from model tests with wave-heights of $0.15 \mathrm{~m}$ and $0.25 \mathrm{~m}$ with water depth $=1.85 \mathrm{~m}$ and weir height $=50 \mathrm{~mm}$

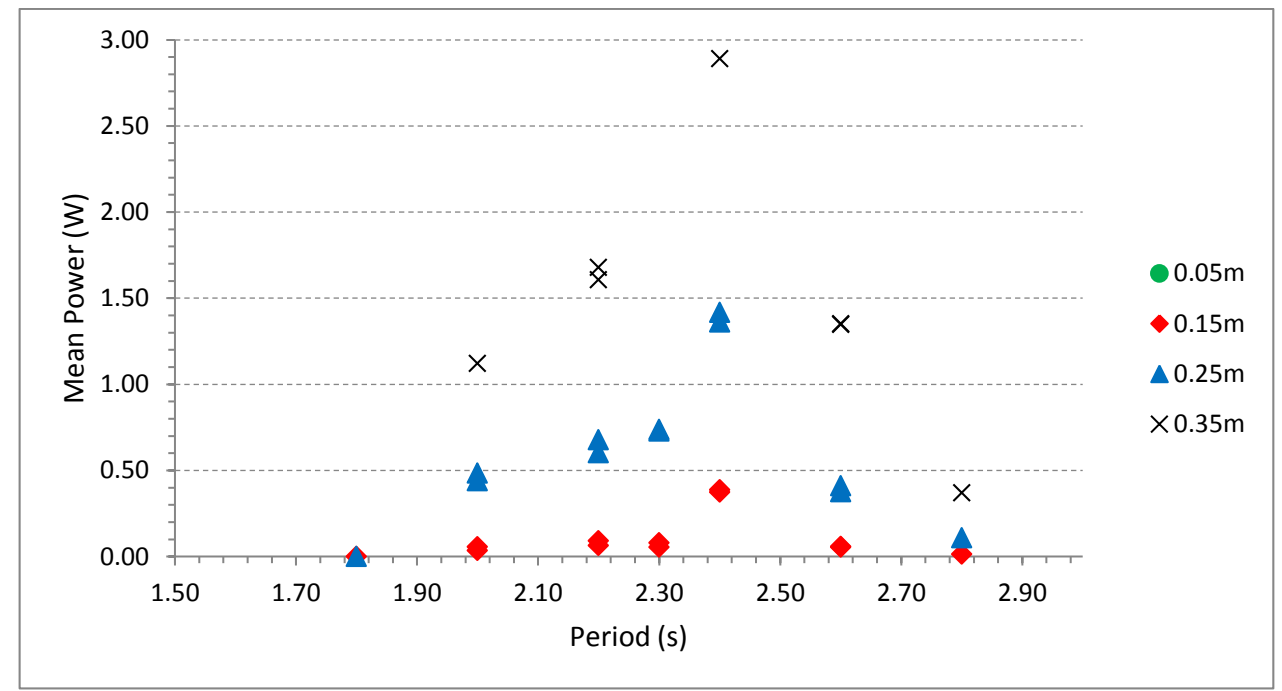

Figure 3: Mean power against wave frequency calculated from model tests with wave-heights of $0.15 \mathrm{~m}, 0.25 \mathrm{~m}$ and $0.35 \mathrm{~m}$ with water depth $=1.85 \mathrm{~m}$ and weir height $=65 \mathrm{~mm}$ 


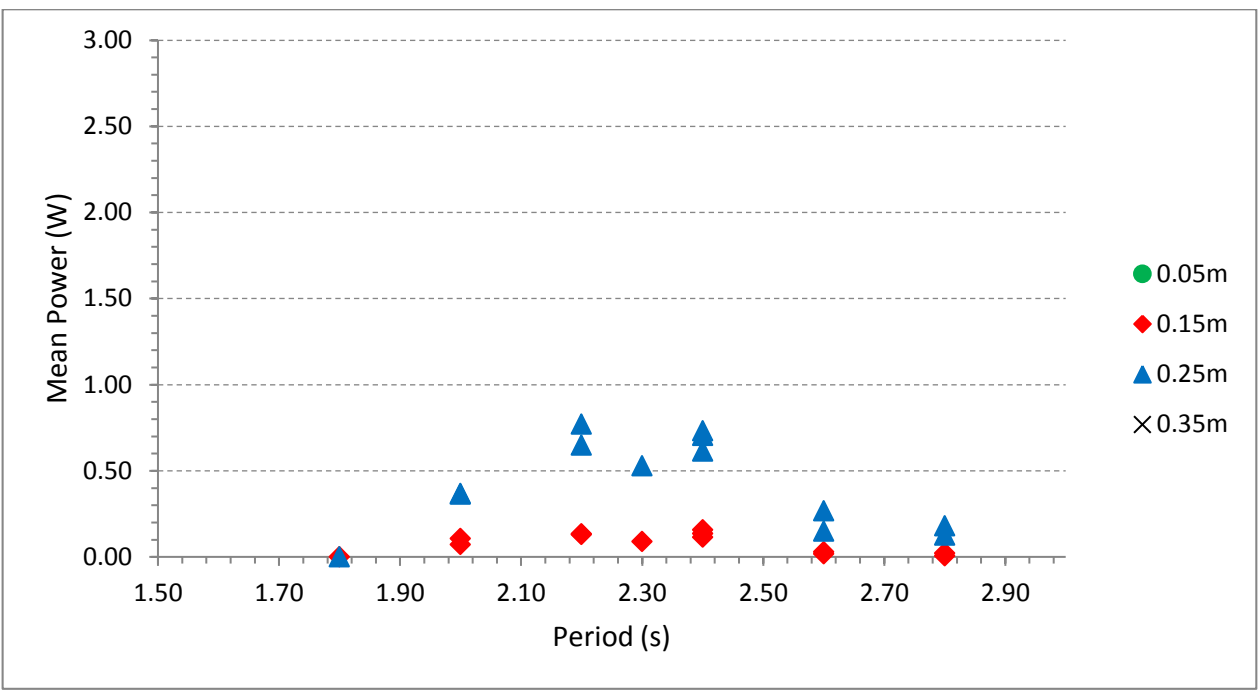

Figure 4: Mean power against wave frequency calculated from model tests with wave-heights of $0.15 \mathrm{~m}$ and $0.25 \mathrm{~m}$ with water depth $=2.35 \mathrm{~m}$ and weir height $=35 \mathrm{~mm}$

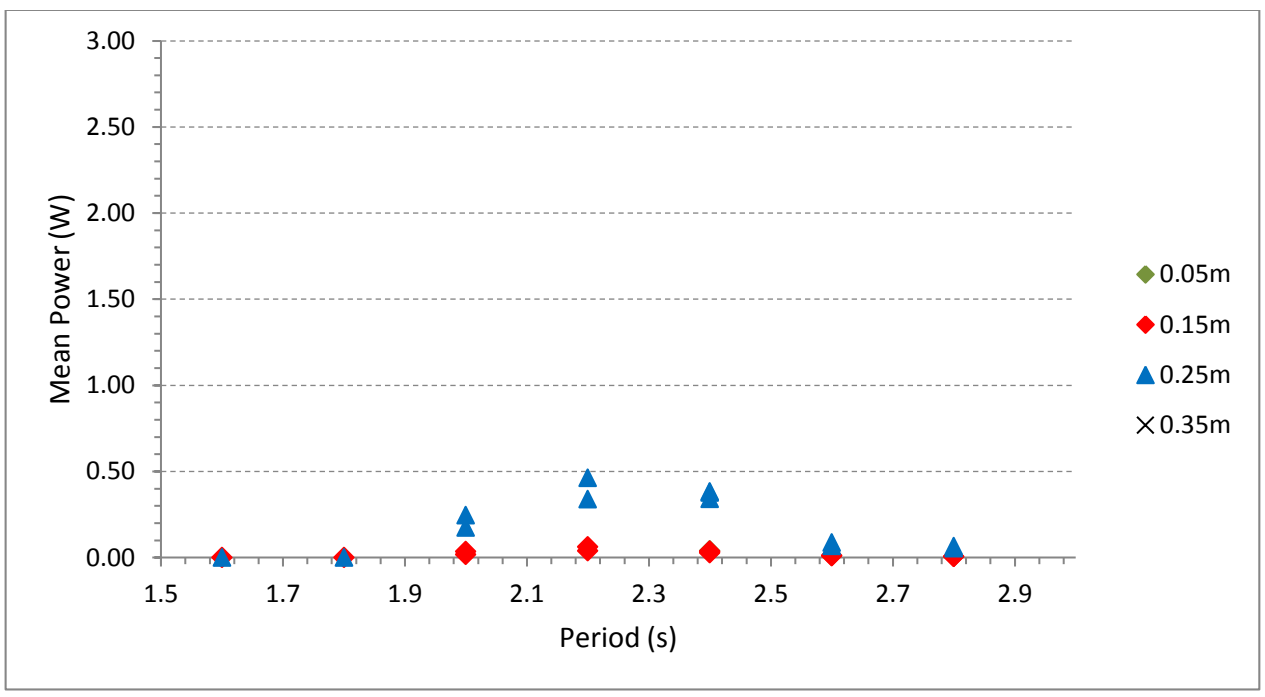

Figure 5: Mean power against wave frequency calculated from model tests with wave-heights of $0.15 \mathrm{~m}$, and $0.25 \mathrm{~m}$ with water depth $=2.35 \mathrm{~m}$ and weir height $=50 \mathrm{~mm}$ 


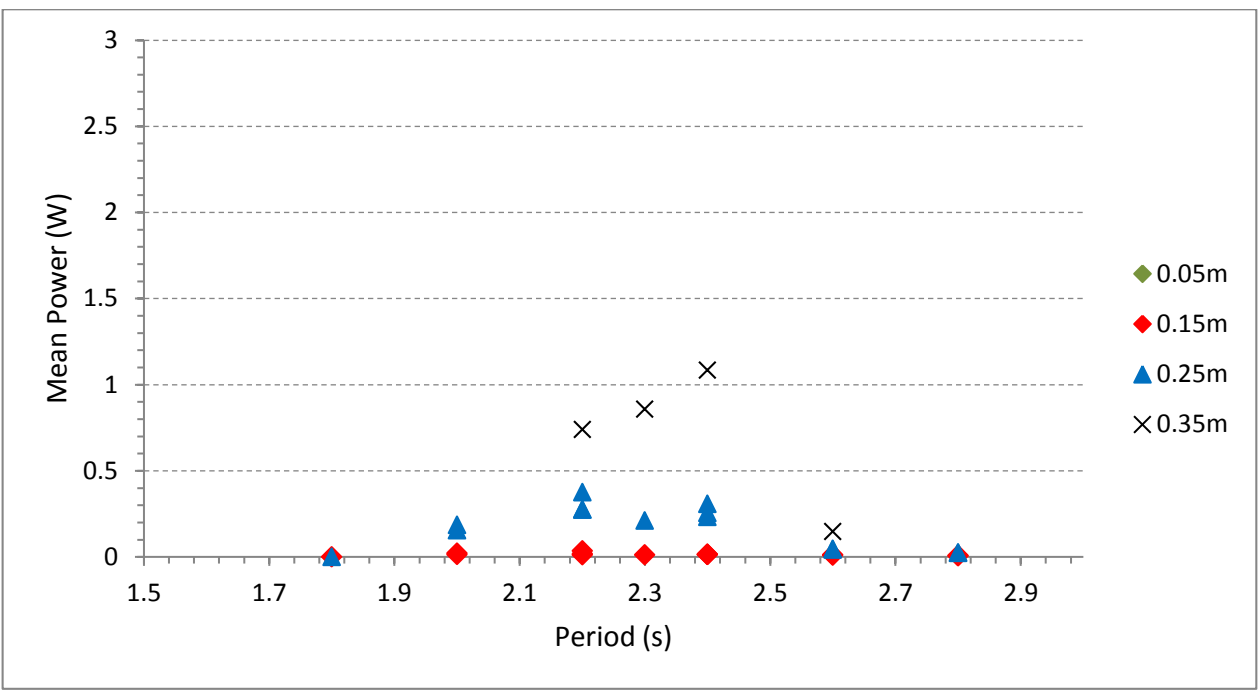

Figure 6: Mean power against wave frequency calculated from model tests with wave-heights of $0.15 \mathrm{~m}, 0.25 \mathrm{~m}$ and $0.35 \mathrm{~m}$ with water depth $=2.35 \mathrm{~m}$ and weir height $=65 \mathrm{~mm}$

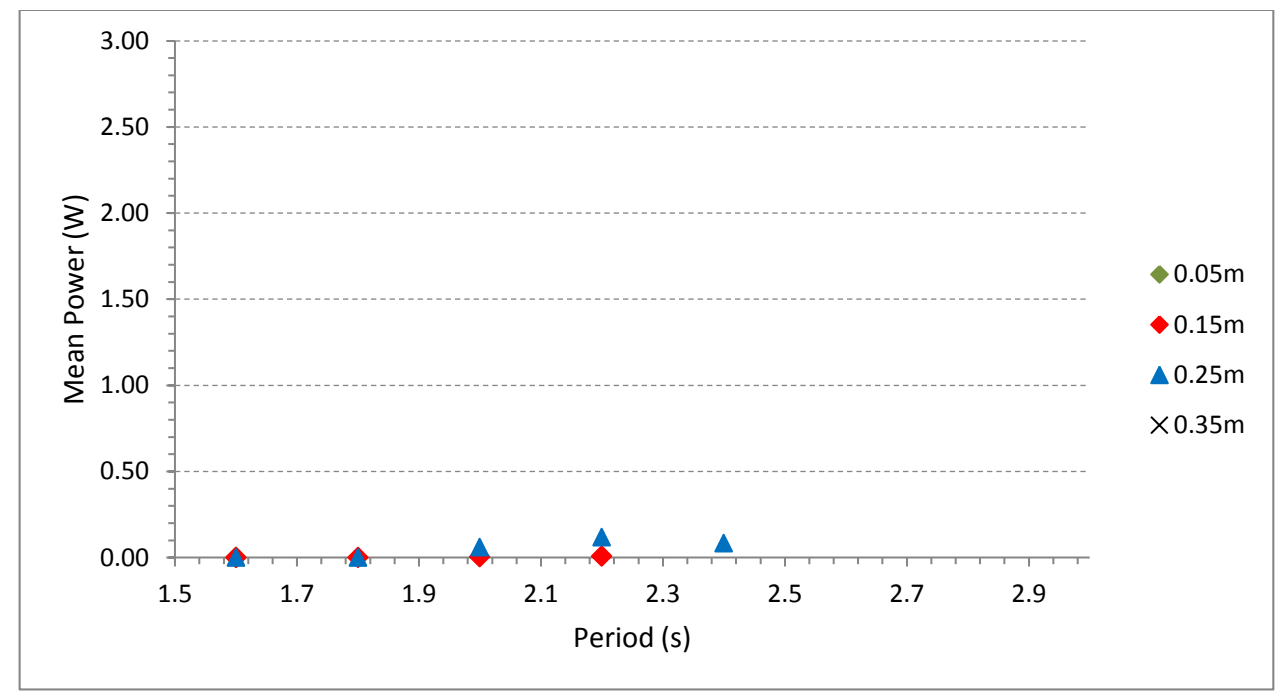

Figure 7: Mean power against wave frequency calculated from model tests with wave-heights of $0.15 \mathrm{~m}$, and $0.25 \mathrm{~m}$ with water depth $=2.75 \mathrm{~m}$ and weir height $=50 \mathrm{~mm}$ 